\title{
Differenzierung der Pharmakotherapie mit Fasudil und Riluzol im SOD1-G93A Mausmodell der Amyotrophen Lateralsklerose
}

\author{
INAUGURAL-DISSERTATION \\ zur Erlangung des Doktorgrades \\ der Medizinischen Fakultät der \\ Georg-August-Universität zu Göttingen
}

vorgelegt von

David Scheer

aus

Göttingen

Göttingen 2018 
Dekan:

Referent/in

Ko-Referent/in:
Prof. Dr. rer. nat. H. K. Kroemer

Prof. Dr. med. L. Tönges

Prof. Dr. rer. nat. B. Reuss

Datum der mündlichen Prüfung: 05.12.2018 
Hiermit erkläre ich, die Dissertation mit dem Titel "Differenzierung der Pharmakotherapie mit Fasudil und Riluzol im SOD1-G93A Mausmodell der Amyotrophen Lateralsklerose" eigenständig angefertigt und keine anderen als die von mir angegebenen Quellen und Hilfsmittel verwendet zu haben.

Oldenburg, den 


\section{Inhaltsverzeichnis}

Abbildungsverzeichnis ....................................................................................... III

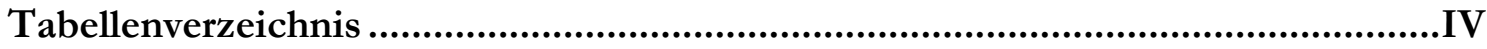

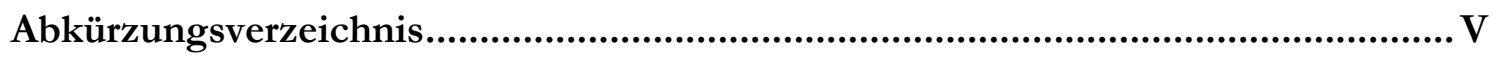

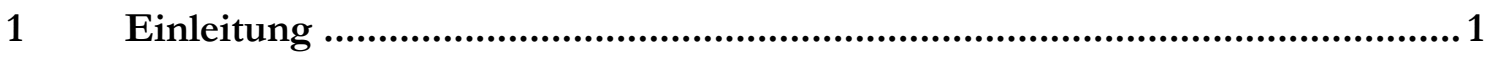

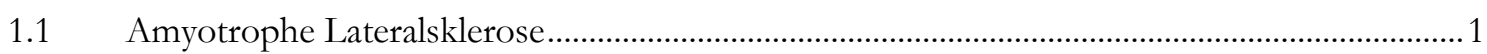

1.1.1 Definition, Begrifflichkeit und Geschichte …………...........................................................

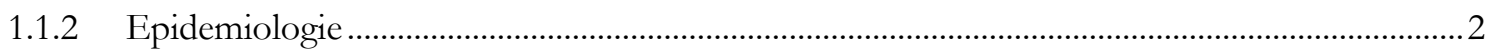

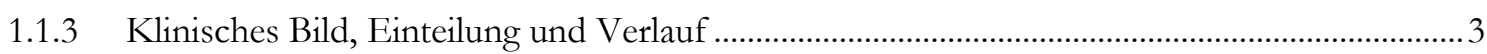

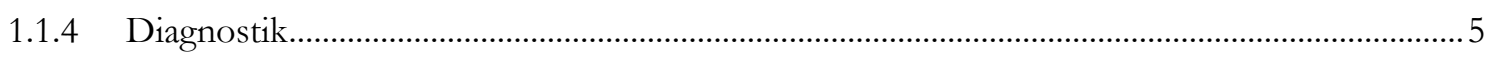

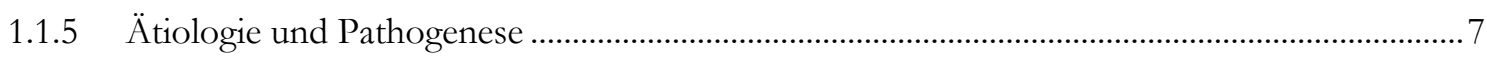

1.1.6 Therapie und Prognose ………………………...........................................................

1.2 Das High-copy-B6SJL-Tg(SOD1-G93A)1Gur/J-Mausmodell ..................................................12

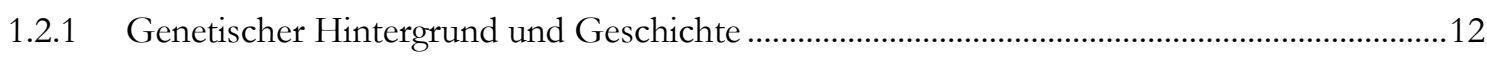

1.2.2 Phänotyp: Klinischer Verlauf und Pathophysiologie...........................................................13

1.2.3 Verhaltensbiologische Methoden der Krankheitsbeobachtung.................................................14

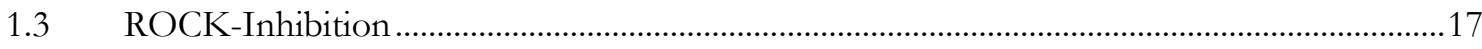

1.3.1 ROCK1 und ROCK2: Rho-assoziierte Coiled-Coil containing Proteinkinasen.......................17

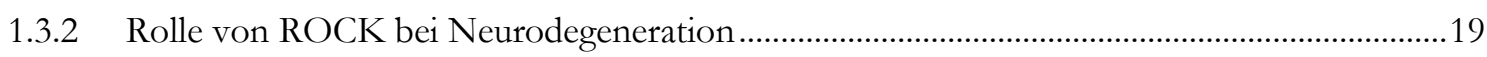

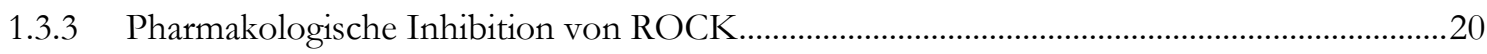

1.3.4 ROCK-Inhibition in Modellen der Neurodegeneration - ein Überblick.................................20

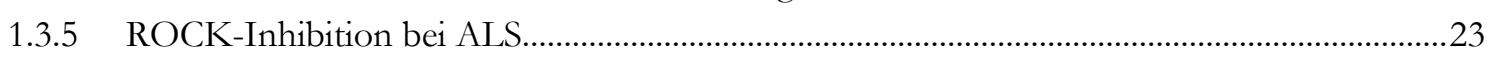

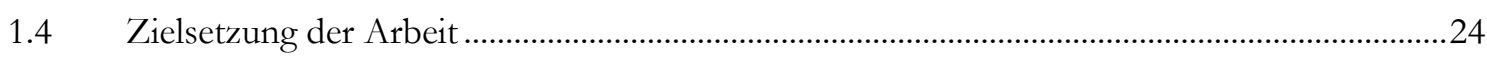

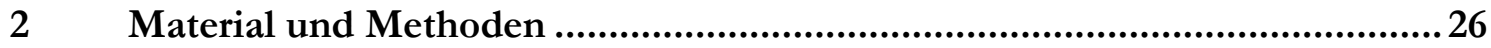

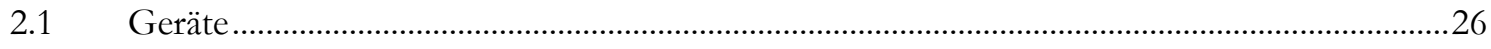

$2.2 \quad$ Verbrauchsmaterialien und allgemeine Chemikalien ...............................................................2

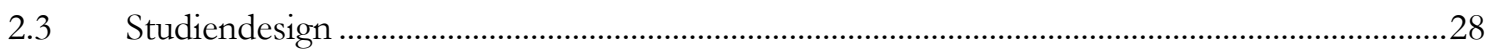

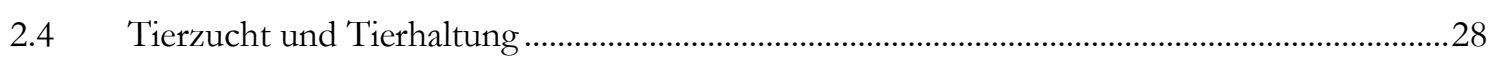

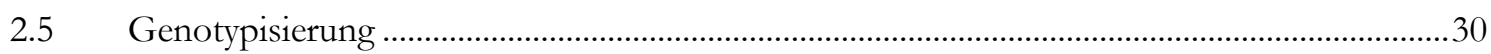

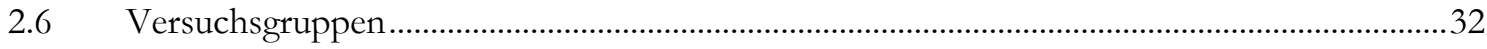

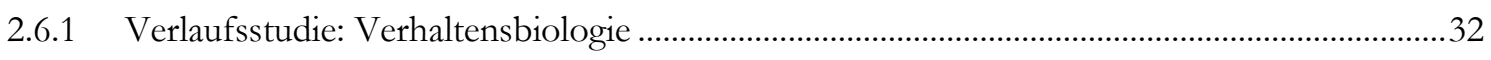

2.6.2 Querschnittsstudie: Evaluation der spinalen Motoneurone und der Muskelatrophie ............33

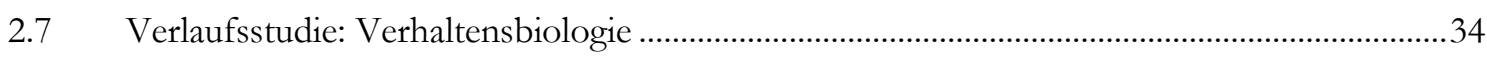

2.7.1 Klinische Analyse: Überleben, Krankheitsverlauf und Körpergewicht.......................................34

2.7.2 Analyse der Motorkoordination mittels Drehwalzentest (Rotarod),.........................................36

2.7.3 Videobasierte Analyse des Gangs bei sich frei bewegenden Mäusen (CatWalk XT) ..............37

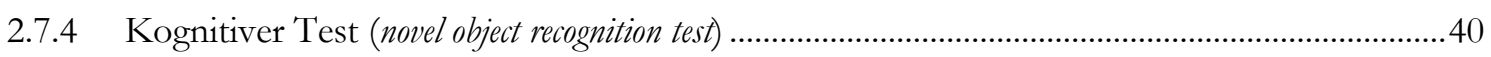


2.8 Querschnittstudie: Evaluation der spinalen Motoneurone und der Muskelatrophie .............42

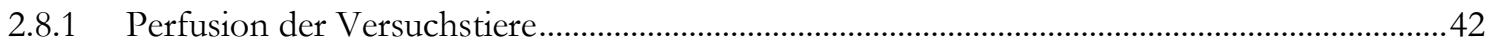

2.8.2 Evaluation der Atrophie des Musculus gastrocnemius ..............................................................43

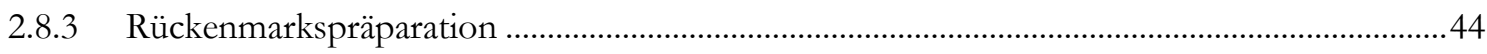

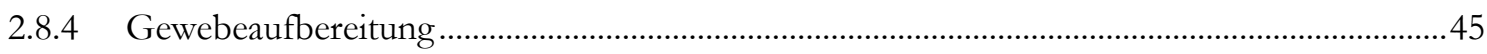

2.8.5 Immunhistochemische Darstellung der Alpha-Motoneurone ..................................................45

2.8.6 Mikroskopie und Auswertung der Motoneurone....................................................................4

2.8.7 Statistische Auswertung der histologisch gewonnenen Daten .............................................47

$3 \quad$ Ergebnisse....................................................................................... 48

3.1 Verlaufsstudie unter präsymptomatischer Behandlung mit Fasudil und Riluzol ab

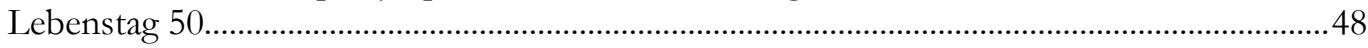

3.1.1 Klinische Analyse: Überleben, Krankheitsverlauf und Körpergewicht.................................48

3.1.2 Motorische Verhaltensanalyse (Drehwalzentest) ..................................................................54

3.1.3 Videobasierte Analyse des Gangs bei sich frei bewegenden Mäusen (CatWalk XT) .............56

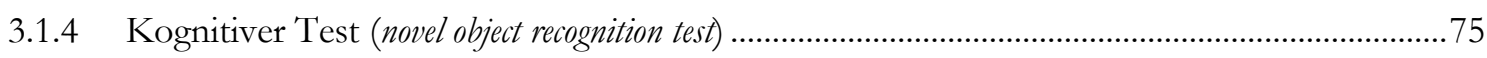

3.2 Querschnittstudie nach präsymptomatischer Behandlung mit Fasudil und Riluzol ab

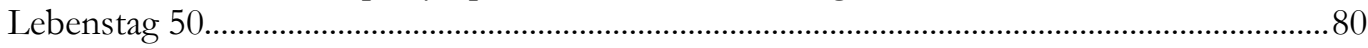

3.2.1 Evaluation der Atrophie des Musculus gastrocnemius ........................................................8

3.2.2 Histologische Evaluation der spinalen Alpha-Motoneurone ..................................................8 81

$4 \quad$ Diskussion.......................................................................................... 84

4.1 Verlaufsstudie unter präsymptomatischer Behandlung mit Fasudil und Riluzol ...................84

4.1.1 Kein Überlebensvorteil - Applikationsdauer von Bedeutung .................................................8 84

4.1.2 Kein modifizierender Einfluss auf den Krankheitsverlauf ......................................................85

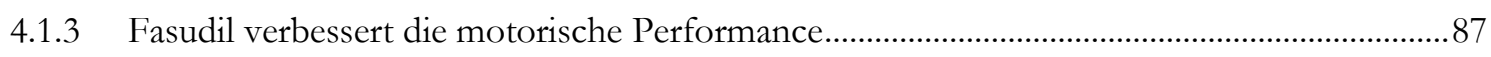

4.1.4 SOD1G93A-Mausmodell und kognitive Verhaltensbiologie.................................................93

4.2 Querschnittstudie nach präsymptomatischer Behandlung mit Fasudil und Riluzol ..............94

4.2.1 Protektive Effekte auf die Muskelatrophie durch Fasudil und Riluzol ..................................95

4.2.2 Unbeeinflusster Verlust der Alpha-Motoneurone im lumbalen Rückenmark.......................98

4.2.3 Keine Synergieeffekte durch Kombination mit niedrig dosiertem Fasudil............................100

4.3 Fazit zur Therapie mit niedrig dosiertem Fasudil und in Kombination mit Riluzol ............101

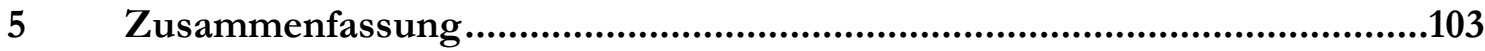

6 Literaturverzeichnis .........................................................................105 


\section{Abbildungsverzeichnis}

Abbildung 1. Diagnosestellung nach den El-Escorial-Kriterien..........................................................6

Abbildung 2 Überblick über die Pathomechanismen der ALS ..........................................................9

Abbildung 3. Genotypisierung der SOD1G93A-transgenen Tiere .....................................................32

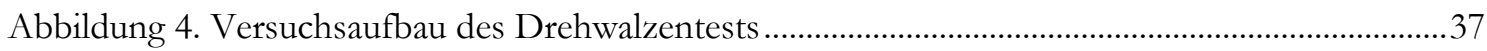

Abbildung 5. Videobasierte Ganganalyse mit dem CatWalk XT gait analysis system ............................38

Abbildung 6. Gangparameter der videobasierten Ganganalyse (CatWalk XT) .................................39

Abbildung 7. Versuchsaufbau des kognitiven NOR-Tests.................................................................41

Abbildung 8. Überlebensanalyse der Behandlungsgruppen ................................................................49

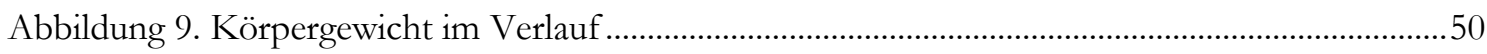

Abbildung 10. Körpergewicht im Vergleich zum Anfangsgewicht..................................................51

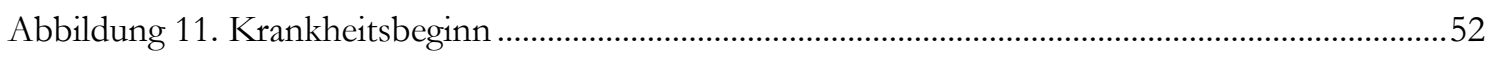

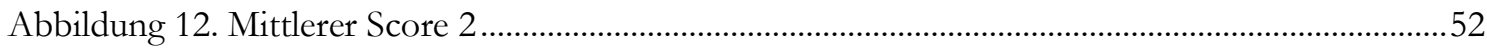

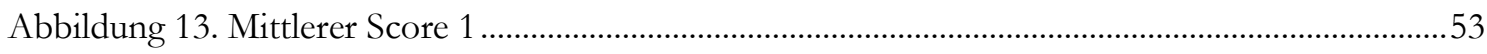

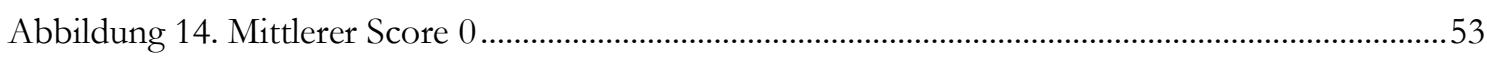

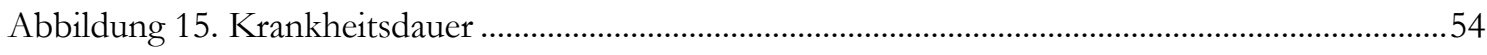

Abbildung 16. Drehwalzenversuch: Mittlere Laufzeit....................................................................56

Abbildung 17. Videobasierte Ganganalyse: Durchschnittsgeschwindigkeit .......................................58

Abbildung 18. Durchschnittsgeschwindigkeit am Tag 127 ..............................................................59

Abbildung 19. Videobasierte Ganganalyse: Auftrittsfläche rechter Vorderlauf.................................60

Abbildung 20. Auftrittsfläche rechter Vorderlauf am Tag 127 .......................................................61

Abbildung 21. Videobasierte Ganganalyse: Auftrittsfläche rechter Hinterlauf .................................62

Abbildung 22. Auftrittsfläche rechter Hinterlauf am Tag 127 ........................................................63

Abbildung 23. Videobasierte Ganganalyse: Auftrittslänge rechter Vorderlauf ..................................64

Abbildung 24. Videobasierte Ganganalyse: Auftrittslänge rechter Hinterlauf ....................................65

Abbildung 25. Auftrittslänge rechter Hinterlauf am Tag 127 ............................................................66

Abbildung 26. Videobasierte Ganganalyse: Schrittgeschwindigkeit rechter Vorderlauf....................68

Abbildung 27. Schrittgeschwindigkeit rechter Vorderlauf am Tag 127 ............................................69

Abbildung 28. Videobasierte Ganganalyse: Schrittgeschwindigkeit rechter Hinterlauf......................70

Abbildung 29. Schrittgeschwindigkeit rechter Hinterlauf am Tag 127 .............................................71

Abbildung 30. Videobasierte Ganganalyse: Schrittlänge rechter Vorderlauf.....................................72

Abbildung 31. Schrittlänge rechter Vorderlauf am Tag 127 .................................................................73

Abbildung 32. Videobasierte Ganganalyse: Schrittlänge rechter Hinterlauf.......................................74

Abbildung 33. Schrittlänge rechter Hinterlauf am Tag 127 .............................................................75

Abbildung 34. Kognitiver Test (novel object recognition test): Zurückgelegte Strecke.................................77

Abbildung 35. Kognitiver Test (novel object recognition test): discrimination index.......................................79

Abbildung 36. Anteil des Musculus gastrocnemius am Körpergewicht an Lebenstag 120 ................81

Abbildung 37. ChAT-Staining: Anzahl der Alpha-Motoneurone/Vorderhorn im Lumbalmark .......82

Abbildung 38. Alpha-Motoneurone/Vorderhorn am Lebenstag 120 ................................................83 


\section{Tabellenverzeichnis}

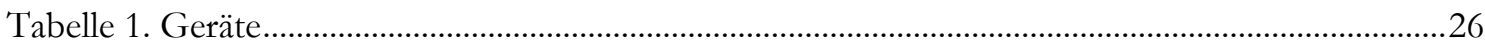

Tabelle 2. Verbrauchsmaterialien und allgemeine Chemikalien..........................................................27

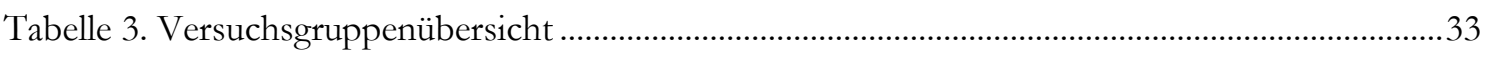

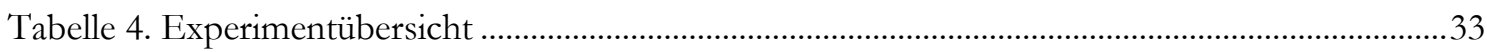

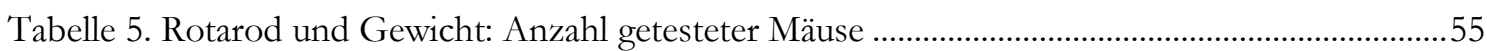

Tabelle 6. Videobasierte Ganganalyse (CatWalk XT): Anzahl durchgeführter Testläufe......................57

Tabelle 7. Kognitiver Test: Anzahl aufgenommener und ausgewerteter Videos....................................76 


\section{Abkürzungsverzeichnis}

AD

ALS

ANOVA

bp

EAAT2

EAE

fALS

Fas10

Fas10Ril30

Fas30

FELASA

FTLD

ERK

GFAP

GLT1

HD

$\mathrm{Htt}$

LIMK

LSD-Test

MBS

MLC

MLCP

MND

MOG

MS

NF-L

pAkt

PBS

PD

PFA

PLP
Morbus Alzheimer/Alzeimer's Disease

Amyotrophe Lateralsklerose

analysis of variance / Varianzanalyse

Basenpaare

excitatory amino acid transporter 2

experimental autoimmune encephalomyelitis

familiäre Amyotrophe Lateralsklerose

Behandlungsgruppe transgener Tiere mit Fasudil 10 mg/kg KG/d

Behandlungsgruppe transgener Tiere mit Kombination aus Fasudil $10 \mathrm{mg} / \mathrm{kg} \mathrm{KG} / \mathrm{d}$ und Riluzol 30 mg/kg KG/d

Behandlungsgruppe transgener Tiere mit Fasudil 30 mg/kg KG/d

Federation of European Laboratory Animal Science Associations

Frontotemporale Lobärdegeneration

extracellular signal-regulated kinase

Saures Gliafaserprotein

glutamate transporter 1 (entspricht EAAT2)

Chorea Huntington/Huntington's Disease

Huntingtin

LIM-Kinase

Least Significance Difference Test

myosin-binding site

myosin-II regulatory light chain

Myosin-Leichte-Ketten-Phosphatase

motoneuron disease

myelin oligodendrocyte glycoprotein

Multiple Sklerose

Neurofilamentprotein

phosphoryliertes Akt

Phosphate-buffered saline

Morbus Parkinson/Parkinson's Disease

Paraformaldehyd-Lösung

proteolipid protein 
pTDP-43 phosphoryliertes TAR-DNA-binding protein-43

PTEN phosphatase and tensin homolog

Ril30 Behandlungsgruppe transgener Tiere mit Riluzol 30 mg/kg KG/d

ROCK Rho-assoziierte Coiled-Coil containing Proteinkinase

sALS sporadische Amyotrophe Lateralsklerose

SMA Spinale Muskelatrophie

Smn-1 survival of motoneuron-1

SOD Superoxiddismutase

SOD1 Cu-/Zn-Superoxiddismutase

SOD1G93A Cu-/Zn-Superoxiddismutase mit der Punktmutation G93A

Stdf Standardfehler

Veh Vehikel, Gruppe transgener Tiere ohne Behandlung

Wt Wildtyp-Kontrollgruppe

ZTE Zentrale Tierexperimentelle Einrichtung der Universitätsmedizin Göttingen 


\section{$1 \quad$ Einleitung}

\subsection{Amyotrophe Lateralsklerose}

\subsubsection{Definition, Begrifflichkeit und Geschichte}

Die amyotrophe Lateralsklerose (ALS) ist eine neurodegenerative Erkrankung der Motoneurone (Rowland und Shneider 2001), bei der sowohl das erste Motoneuron des kortikospinalen Trakts als auch das zweite im Vorderhorn des Rückenmarks oder in der Medulla oblongata (Bulbus) betroffen sind (Talbot und Ansorge 2006). Muskelschwäche und Atrophie sind neben Faszikulationen kennzeichnende Symptome der Denervierung in den betroffenen Muskelpartien - beginnend häufig peripher in den kleinen Handmuskeln durch den Untergang des zweiten Motoneurons. Letztendlich kommt es durch die Beteiligung der Atemmuskulatur zu einer zunehmenden respiratorischen Insuffizienz, die insbesondere durch die erhöhte Vulnerabilität für Atemwegsinfektionen den Tod des Erkrankten zur Folge haben kann und deshalb als die Haupttodesursache im Rahmen der infaust verlaufenden ALS gilt (Ludolph 2006). Durch die Schädigung des ersten Motoneurons kommt es zu Hyperreflexie, Kloni, Spastiken und Pyramidenbahnzeichen in den betroffenen Gebieten (Rowland und Shneider 2001).

Die Bezeichnung „amyotroph“ beschreibt somit bereits den Teil der Klinik, der durch die Degeneration des zweiten Motoneurons begründet ist: die Muskelatrophie. Die Bezeichnung „Lateralsklerose“ bezieht sich auf den verhärtet tastbaren Seitenstrang des Rückenmarks bei autopsierten ALS-Patienten (Rowland und Shneider 2001).

Als Erstbeschreiber eines solchen Krankheitsbildes nennen Tyler und Shefner (1991) den schottischen Anatomen Charles Bell, der als Entdecker des Funktionsunterschiedes von Hinter- und Vorderhorn 1824 über Patienten berichtet, die mit rein motorischen Ausfällen auffielen (Rowland 2001). 1850 beschreibt François-Amilcar Aran weitere Fälle und verwendet dafür den Namen „progressive spinale Muskelatrophie“; dieselben Patienten werden von Guillaume-Benjamin Amand Duchenne elektrophysiologisch untersucht, das entdeckte Krankheitsbild „Aran-Duchenne-Syndrom“ genannt (Aran 1850, Duchenne 1851, Rowland 2001), das heute als progressive Muskelatrophie eine Entität ist, die oft nicht eindeutig von der ALS abgegrenzt werden kann und daher als klinische Variante der ALS ohne Symptome des ersten Motoneurons mehr als 10\% der Fälle ausmacht (Meyer et al. 2007). Im Jahr 1853 beschreibt Jean-Baptiste Cruveilhier als erster die Pathologie der 
ALS. Er beobachtet bei Autopsien eine Atrophie der Vorderhörner und vermutet eine Dysfunktion der Vorderhornzellen als Ursache (Cruveilhier 1853, Rowland 2001).

In Kenntnis dieser Vorarbeiten und auf Grundlage eigener Beobachtungen beschreibt Jean-Martin Charcot zusammen mit Alix Joffroy im Jahre 1869 erstmalig ein Krankheitsbild mit einer Kombination aus Muskelatrophie und spastischen Kontrakturen (Charcot und Joffroy 1869, Rowland 2001), um im Jahre 1874 in Zusammenschau von Klinik und Pathologie den Krankheitsbegriff der ALS zu prägen (Charcot 1874). Auch heute noch - fast hundertfünfzig Jahre später - wird die Diagnose der ALS primär klinisch gestellt und kann erst nach dem Tod des Patienten pathologisch gesichert werden (Leitlinie ALS 2012, Rowland 2001).

\subsubsection{Epidemiologie}

Die ALS zählt mit einer Inzidenz zwischen 1,7 und 2,3/100.000 pro Jahr und mit einer ähnlich hohen Mortalität (Logroscino et al. 2010) zu den seltenen Erkrankungen (Logroscino et al. 2008). Die Prävalenz wird mit um die 6/100.000, das mittlere Erkrankungsalter mit 58-63 Jahren und das mittlere Überleben mit 3-4 Jahren angegeben (Chiò et al. 2013, Talbott 2016). Das Risiko, im Laufe des Lebens an ALS zu erkranken, wird mit Werten zwischen 1:400 und 1:1000 angegeben; in industrialisierten Ländern stirbt immerhin mehr als einer von 500 Menschen an ALS. Männer erkranken jedoch insgesamt in einem Verhältnis von ca. 1,2-1,5/1 etwas häufiger als Frauen an ALS (Hardiman et al. 2011, Andersen et al. 2005, Ludolph et al. 2010). Es kann zudem von einer leichten Zunahme sowohl der Inzidenz als auch der Mortalität im Verlauf der letzten Jahrzehnte gesprochen werden. Dies betrifft vor allem Südeuropa, Frauen und Menschen über 75 Jahre (Logroscino et al. 2008, Román 1996, Chancellor und Warlow 1992). Dies wird mit einer höheren Lebenserwartung und der verbesserten Diagnostik im Rahmen der Entwicklung der El Escorial-Kriterien Mitte der neunziger Jahre in Verbindung gebracht (Logroscino et al. 2010, Worms 2001). Zudem gibt es Anzeichen dafür, dass das Auftreten in Populationen nicht-europäischer Herkunft etwas niedriger ist und auch in ethnisch gemischten Populationen reduziert ist (Zaldivar et al. 2009).

Während die große Mehrheit der Fälle der sporadischen Form (sALS) zugeordnet werden kann, macht die familiäre ALS (fALS), wie familiär gehäufte Formen mit und ohne Nachweis einer Mutation zusammengefasst genannt werden, mindestens ca. 5-10\% der Fälle aus (Talbott 2016). Die fALS ist durch einen deutlich früheren Erkrankungsbeginn bei einem mittleren Erkrankungsalter von 47-52 Jahren gekennzeichnet (Andersen et al. 2005). 16 Genloci mit hunderten möglichen Mutationen lassen sich unzweifelhaft der fALS 
zuordnen und sind in vielen Fällen auch bei der sALS nachweisbar (Taylor et al. 2016). Darüber hinaus sind weitere Genveränderungen bekannt, die als Risikofaktor für die Entwicklung einer sALS gelten (Rübsamen und Lücking 2013). So kann man als Hauptrisikofaktoren für die ALS das männliche Geschlecht, hohes Lebensalter und eine genetische Disposition nennen.

Zudem gibt es zwei epidemiologische Sonderfälle: Zum einen wurde in der Mitte des 20. Jahrhunderts unter den Ureinwohnern der pazifischen Insel Guam - den Chamorros - eine 100-mal höhere Inzidenz der ALS und eine erhöhte Prävalenz von familï̈ren Häufungen gefunden, was bis dato außerhalb Guams nicht beobachtet werden konnte. Es kommen als Erklärung für dieses Phänomen neben genetischen Prädispositionen auch Umweltfaktoren infrage (Lee 2011). Zum anderen gibt es eine Häufung von ALS in der Gruppe der sportlich sehr aktiven Menschen: Es zeigt sich in der Gruppe der Fußballspieler und der American Football-Spieler eine signifikant höhere Anzahl von ALS-Erkrankungen. Dies betrifft vor allem männliche Spieler in Spielpositionen, die hohe Laufleistung und Geschwindigkeit erfordern. Trotzdem bleibt umstritten, ob hohe körperliche Aktivität als ein Risikofaktor für die ALS gelten kann (Lacorte et al. 2016).

\subsubsection{Klinisches Bild, Einteilung und Verlauf}

Maßgeblich für das klinische Bild der ALS ist die Kombination aus Symptomen des ersten Motoneurons und Symptomen des zweiten Motoneurons: das gleichzeitige Auftreten einerseits von Muskelschäche, Muskelatrophie und Faszikulationen und andererseits von Muskelkloni, gesteigerten Muskeleigenreflexen, progressiver Bulbärparalyse und Pyramidenbahnzeichen (Brooks 1994). Die fALS unterscheidet sich dabei klinisch nicht von der sALS, sie fällt allerdings durch einen früheren Krankheitsbeginn auf (Hardiman et al. 2011, Sabatelli et al. 2008). In der überwiegenden Mehrzahl, bei 98\% der Fälle, beginnt die Symptomatik fokal in einer Körperregion (Ravits et al. 2007): bulbär, in den Armen, in den Beinen oder im Rumpf. Daneben ist ein Fortschreiten und ein Ausbreiten der Symptome innerhalb der Region und auch ein Übergreifen auf andere Regionen - eine sogenannte Progression - charakteristisch, weshalb bei Verdacht eine regelmäßige klinischneurologische Untersuchung - wenigstens alle 6 Monate - von unabdingbarer Bedeutung für die klinische Diagnosestellung ist (Brooks 1994). Initialsymptome der ALS sind Muskelkrämpfe und Faszikulationen, jedoch sind sie nicht spezifisch und nicht ausreichend für eine Diagnosestellung (Leitlinie ALS 2012).

Die ALS präsentiert sich klinisch in verschiedenen Formen: In den Extremitäten beginnend und beide Motoneurone betreffend, bulbär beginnend mit Sprech- und 
Schluckbeschwerden auf die Extremitäten übergreifend und die beiden seltenen Phänomene primäre Lateralsklerose, die nur das erste Motoneuron betrifft und ebenso spinal oder bulbär beginnen kann (Pseudobulbärparalye) sowie die progressive Muskelatrophie, die nur das zweite Motoneuron betrifft (Kiernan et al. 2011). Daneben werden seltene Syndrome wie das Vulpian-Bernhard-Syndrom, das auch flail arm-Syndrom genannt wird, und das Kennedy-Syndrom zum Formenkreis der ALS und teils als Subtypen gezählt (Leitlinie ALS 2012).

Die klinische Einteilung der klassischen ALS erfolgt nach dem Symptombeginn: Die überwiegende Mehrzahl - zwischen 60 und 70\% - der Fälle präsentiert sich mit einem spinalen Symptombeginn in den Extremitäten (Kiernan et al. 2011), während die obere Extremität weit häufiger zuerst betroffen ist (Rübsamen und Lücking 2013), etwa 30 \% mit einem bulbären Symptombeginn und ein weiterer Teil der Fälle mit einem unspezifischen Symptombeginn, etwa emotionaler Labilität, Gewichtsverlust oder initialen Atembeschwerden (Logroscino et al. 2010).

Die beiden beschriebenen Einteilungen scheinen angesichts neuer Erkenntnisse auf molekular-genetischer und molekular-pathologischer Ebene nicht geeignet zu sein, die verschiedenen Formen scharf voneinander abgrenzen zu können. Eher kann davon ausgegangen werden, dass die Formen verschiedene Ausprägungen einer systematischen Degeneration sind (Braak et al. 2013), die sich in Stadien ausbreitet (Leitlinie ALS 2012, Brettschneider et al. 2013). Ausgehend von dieser Sichtweise lässt sich ebenfalls einordnen, dass es einen bestimmten Anteil der ALS-Patienten gibt, der Symptome entwickelt, die als frontale Demenz gedeutet werden (Schreiber et al. 2005): Bei etwa 5-15\% der ALSPatienten wird im Verlauf auch eine frontotemporale Demenz mit Degeneration frontaler und temporaler Hirnregionen, eine frontotemporale Lobärdegeneration (FTLD) diagnostiziert (Ringholz et al. 2005), bei zwischen 30 und 50 \% zumindest eine Störung der Frontalhirnfunktion oder eine dementielle Entwicklung. Dies deckt sich mit neuropathologischen Überschneidungen beider Erkrankungen, die nahelegen, beide Erkrankungen könnten verschiedene Ausprägungen derselben ubiquitin-assoziierten Neurodegeneration sein (Talbot und Ansorge 2006). In den letzten Jahren verdichtete sich diese Sichtweise durch weitere neuropathologische Beobachtungen, die zeigen, dass sowohl in der FTLD als auch in der ALS ubiquitin-positive phosphorylierte TAR-DNA-binding protein-43 (pTDP-43)-Einschlüsse in den betroffenen Regionen des ZNS gefunden werden können. Durch diese sich überschneidende Pathologie und Klinik werden beide Erkrankungen immer näher miteinander verknüpft. Insbesondere die sich ausbreitende Symptomatik in Verbindung mit der bisher ungeklärten Frage, ob pTDP-43-Einschlüsse 
sich wie Prionen über synaptische Verbindungen ausbreiten können, ist Gegenstand intensiver Forschungsbemühungen (Braak et al. 2013, Taylor et al. 2016).

Die Ausbreitung der Erkrankung von einer Körperregion aus wird klinisch in einem Übergreifen auf andere Körperregionen und durch eine Verschlechterung in der bereits betroffenen Region sichtbar; der Schweregrad der Erkrankung in der sogenannten Amyotrophic Lateral Sclerosis Functional Rating Scale, kurz ALSFRS, eingeordnet (Cedarbaum et al. 1997), welche 1996 entwickelt und 1999 revidiert wurde, um genauere Angaben zur Respiration des Patienten zu ergänzen (ALSFRS-R) (Cedarbaum et al. 1999).

Die Patienten entwickeln im Laufe der Erkrankung Sprach- und Sprechstörungen, unkontrollierbaren Speichelfluss, Schluckstörungen, Schreibstörungen, Probleme bei der Nahrungsaufnahme, bei der Körperpflege, bei der Lagerung im Bett, beim Gehen, beim Treppensteigen und bei der Atmung. Anhand dieser zehn (ALSFRS) bzw. zwölf (ALSFRSR) Items wird durch eine vergebene Punktzahl zwischen 0 und 4 Punkten der Schweregrad der gesamten Erkrankung angegeben.

Betroffen von der Erkrankung ist letztendlich fast die gesamte Steuerung der Willkürmotorik mit zwei Ausnahmen: Sehr selten betroffen sind die Okulomotorik und die Steuerung der Sphinkter der Ausscheidungsorgane. Diese Beobachtung wurde im Rahmen von Autopsien neuropathologisch untersucht und in den betroffenen Kernregionen keine Degeneration der verantwortlichen Motoneurone gefunden; weder in den Kernen der für die Okulomotorik verantwortlichen Hirnnerven (Kaminski et al. 2002) noch im sogenannten Onuf-Kern, den im Vorderhorn des zweiten Sakralsegment gelegen, eine Ansammlung von Neuronen bildet, welche die Sphinktere der Beckenregion innervieren (Mannen 2000, Schroder und Reske-Nielsen 1984). Einzelne Fallstudien legen nahe, dass bei sehr langem Überleben auch diese Neurone letztendlich in die Krankheitsausbreitung einbegriffen werden (Hayashi und Kato 1989).

Die zunehmende Verschlechterung führt letztendlich $\mathrm{zu}$ Immobilisation bis hin zu vollständiger Bettlägerigkeit und Pflegebedürftigkeit, zur Unfähigkeit, selbstständig mit der Umwelt zu kommunizieren und endet mit der Entwicklung einer respiratorischen Insuffizienz aufgrund der Mitbeteiligung der Atemmuskulatur letal infolge von Atemversagen, das häufig mit einer Pneumonie einhergeht (Kiernan et al. 2011).

\subsubsection{Diagnostik}

Um die Diagnose ALS zu stellen, bedarf es einer ausführlichen klinischen Untersuchung des Patienten, da die Diagnose zuvorderst anhand klinischer Kriterien gestellt wird (Leitlinie ALS 2012). 
Entscheidend für die Diagnosestellung ist das gleichzeitige Auftreten von Zeichen des ersten Motoneurons in Kombination mit Zeichen des zweiten Motoneurons. In der Vergangenheit wurden von der World Federation of Neurology (WFN) Research Subgroup on ALS/MND klinische Kriterien entwickelt, um eine sichere Diagnosestellung und insbesondere auch die Einordnung von Patienten in Studien zu ermöglichen. So wurden 1994 die El-Escorial-Kriterien entwickelt, die mehrfach revidiert bzw. ergänzt bis heute Grundlage der Diagnosestellung sind. Sie unterscheiden zwischen einer sicheren ALS, einer wahrscheinlichen ALS, einer wahrscheinlichen laborunterstützten ALS und einer möglichen ALS (Brooks 1994). Da diese Formulierungen in der klinischen Praxis oftmals verwirrend für Patienten und behandelndes Personal sein können, sollten die Kriterien in der klinischen Praxis zumindest restriktiv verwendet werden oder durch die notwendigen Erläuterungen ergänzt werden (Leitlinie ALS 2012). Sie sollten vielmehr tatsächlich nur genutzt werden, um Patienten in klinischen Studien richtig einzuordnen.

Die Symptome des Patienten werden im Rahmen der Kriterien in vier Körperregionen eingeordnet: bulbär, zervikal, thorakal und lumbosakral. In diesen Regionen werden die Symptome des Patienten beurteilt und mit apparativen Untersuchungen zum Ausschluss anderer Ursachen ergänzt. Entsprechend den Befunden kann die Diagnose daraufhin gestellt werden (siehe Abbildung 1).

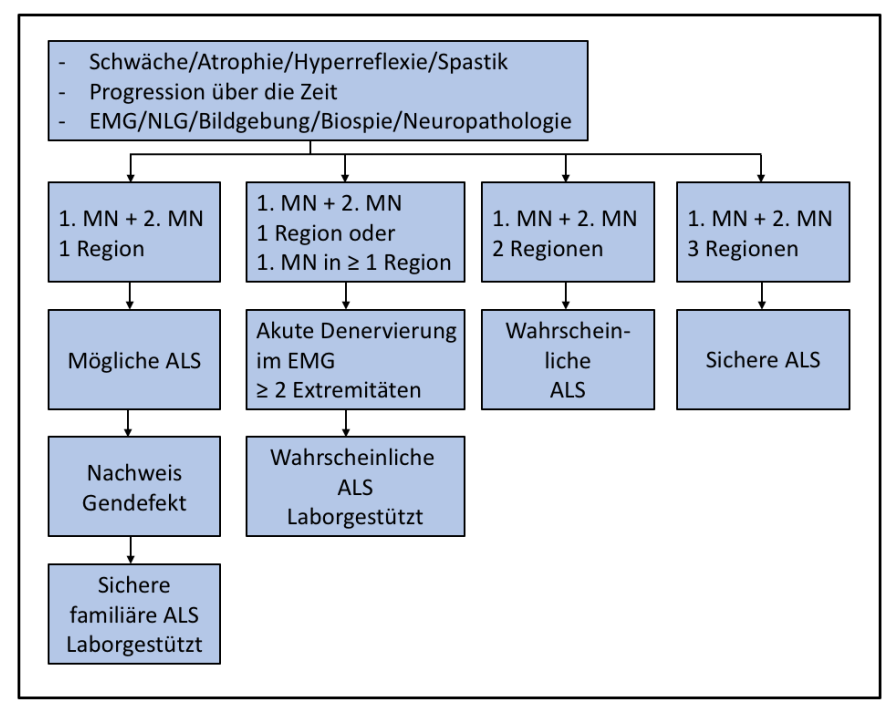

Abbildung 1. Diagnosestellung nach den El-Escorial-Kriterien

ALS: Amyotrophe Lateralsklerose, EMG: Elektromyographie, MN: Motoneuron, NLG: Nervenleitgeschwindigkeit. Abbildung entnommen aus: Körner S et al. 2011, S. 757, mit freundlicher Genehmigung der Autorin und Schattauer $\mathrm{GmbH}$.

Es konnte gezeigt werden, dass die diagnostische Sensitivität erhöht wird, ergänzt man zu den klinisch erhobenen Befunden elektrophysiologisch erhobene Befunde über die Denervierung (de Carvalho et al. 2008). So wurden die den El-Escorial-Kriterien - nach der Revision 1998 auch Airlie-House-Kriterien genannt - (Brooks et al. 2000) ähnlichen Awaji- 
Kriterien entwickelt, die elektrophysiologische Befunde über die Denervierung im EMG mit in die Diagnosestellung einbeziehen und so eine frühere Diagnosestellung erlauben (Costa et al. 2012).

Allerdings sind durch das gewonnene Verständnis der ALS in den letzten Jahren auch im Zusammenhang mit der FTLD und der Ätiopathogenese beider Erkrankungen im Einzelnen und im Zusammenhang die El-Escorial-Kriterien erneut in die Kritik geraten (Agosta et al. 2014) und in den Jahren 2014 und 2015 durch die WFN Research Subgroup on ALS/MND diskutiert worden. Als Ergebnis dieser Diskussion sind folgende Ergebnisse publiziert worden (Ludolph et al. 2015): Die Basis der Diagnose sollte weiterhin der Ausschluss anderer Ursachen für die Symptome sein, welche im Rahmen der ALS eine klinische Progression vorweisen müssen. Ausgehend davon werden zwei Konstellationen genannt, die mindestens vorliegen müssen, um die Diagnose zu stellen: Wenigstens in einer Extremität oder Körperregion sollen progressive Symptome des ersten und zweiten Motoneurons vorliegen - entspricht den El-Escorial-Kriterien für die mögliche ALS - oder klinische Symptome der Denervierung (zweites Motoneuron) in einer Körperregion bzw. elektrophysiologisch nachgewiesene Denervierung im EMG in zwei Körperregionen (bulbär, zervikal, thorakal oder lumbosakral). Elektrophysiologisch müssen neurogene Potentiale, Fibrillationen und/oder positive scharfe Wellen im EMG zweifelsfrei nachgewiesen werden. Mit in diese Diagnosekriterien zählen die folgenden Erkrankungen und sind als seltene Phänotypen der ALS zu sehen, mutmaßlich sind sie besondere Verlaufstypen und schließen die Diagnose ALS somit ein: die Progressive Bulbärparalyse, das flail arm- (Vulpian Bernhard) bzw. flail leg-Syndrom, die Progressive Muskelatrophie und die Primäre Lateralsklerose. Es wird empfohlen, die früheren Bezeichnungen von möglicher ALS bis hin zu sicherer ALS in ein zu entwickelndes Staging-System einfließen zu lassen. Zusätzlich weisen die Autoren darauf hin, dass bis zu 50\% der Patienten im Laufe der Erkrankung eine kognitive Beeinträchtigung zeigen, und sie empfehlen, in neues Staging-System kognitive Parameter miteinzubeziehen. So kann die Diagnose ALS seitdem dort gestellt werden, wo bislang nach den El-Escorial-Kriterien nur eine mögliche ALS diagnostiziert wurde (siehe Abbildung 1).

\subsection{5 Ätiologie und Pathogenese}

Die genauen Ursachen der ALS sind trotz vielen bereits bekannten Mutationen und dem hinzugewonnenen Verständnis der letzten Jahre auf der Ebene der Ätiopathogenese immer noch weitgehend unklar. Daher kann man von einem Dreiklang wichtiger Faktoren ausgehen: Genetik, Umwelteinflüsse und Zeit (Al-Chalabi und Hardiman 2013). 
Al-Chalabi und Hardiman (2013) entwerfen in ihrem Review ein Modell, das diese drei Faktoren einschließt: Danach ist jedem aufgrund einer genetischen Prädisposition ein gewisses Grundrisiko gegeben, an ALS zu erkranken. Durch einen unweigerlich stattfindenden Zellschaden im Laufe des Lebens und durch Umwelteinflüsse setzt an einem bestimmten Punkt ein sich selbst verstärkender Prozess der Neurodegeneration ein. Bei Erkrankten ist dabei eine individuelle Konstellation aus monogenetischen, oligo- bzw. polygenetischen Ursachen, dem Zellschaden über die Zeit und den Umwelteinflüssen, die sich untereinander stark beeinflussen können, als Ursache für den Beginn der dann nicht mehr aufzuhaltenden Neurodegeneration zu sehen.

Als erste genetische Ursache für die fALS wurde die autosomal-dominant vererbte Mutation im Gen der Superoxiddismutase 1 (SOD1) gefunden (Rosen et al. 1993). Die über 150 heute bekannten Mutationen auf der SOD1 machen nach heutigen Erkenntnissen ca. 20\% der fALS aus. Dennoch basiert ein Großteil der bisherigen präklinischen Medikamenten-Studien auf transgenen Mausmodellen mit Mutationen auf diesem Gen, wobei vor allem das in der vorliegenden Studie verwendete SOD1G93A-Modell eine Rolle spielt. Eine Mehrheit der fALS macht aus heutiger Sicht die auch mit der FTLD assoziierte chromosome 9 open reading frame 72 (C9ORF72) -Mutation aus, die sich durch eine GGGGCCrepeat-Expansion auszeichnet, aber weltweit höchst unterschiedlich verteilt ist (Renton et al. 2011, Al-Chalabi und Hardiman 2013). Die dritte wichtige Mutation, die hier zu nennen ist, ist die im für TDP-43 kodierenden TAR-DBP-Gen, weil ihre Entdeckung entscheidenden Einfluss auf das Verständnis der Pathogenese der ALS hatte. So konnten pTDP-43Einschlüsse als wesentlicher Teil des intrazellulären Krankheitsverlaufs identifiziert werden und eine weitere Parallele zur FTLD gezogen werden (Sun und Chakrabartty 2017). Insgesamt werden mittlerweile 16 Genloki beschrieben, die zweifelsfrei mit der ALS assoziiert sind (Taylor et al. 2016).

Naturgemäß weniger eindeutig ist die Studienlage zu Umwelteinflüssen, die für die ALS ursächlich sein können. Mit wechselnder Reproduzierbarkeit nennen Studien neben Leistungssport (siehe Kapitel 1.1.2) unter anderen Rauchen (Armon 2009), Belastung mit Pestiziden (Sutedja et al. 2009), Militärdienst (Weisskopf et al. 2005) und Cyoanotoxine (Bradley und Mash 2009).

Der unaufhaltsame Prozess der Neurodegeneration ist pathomechanisch vergleichbar vielschichtig wie die beschriebene Fülle an ursächlichen Prozessen, die ihn auslösen. Abbildung 2 zeigt einen schematischen Überblick über die bisher erforschten Pathomechanismen. 


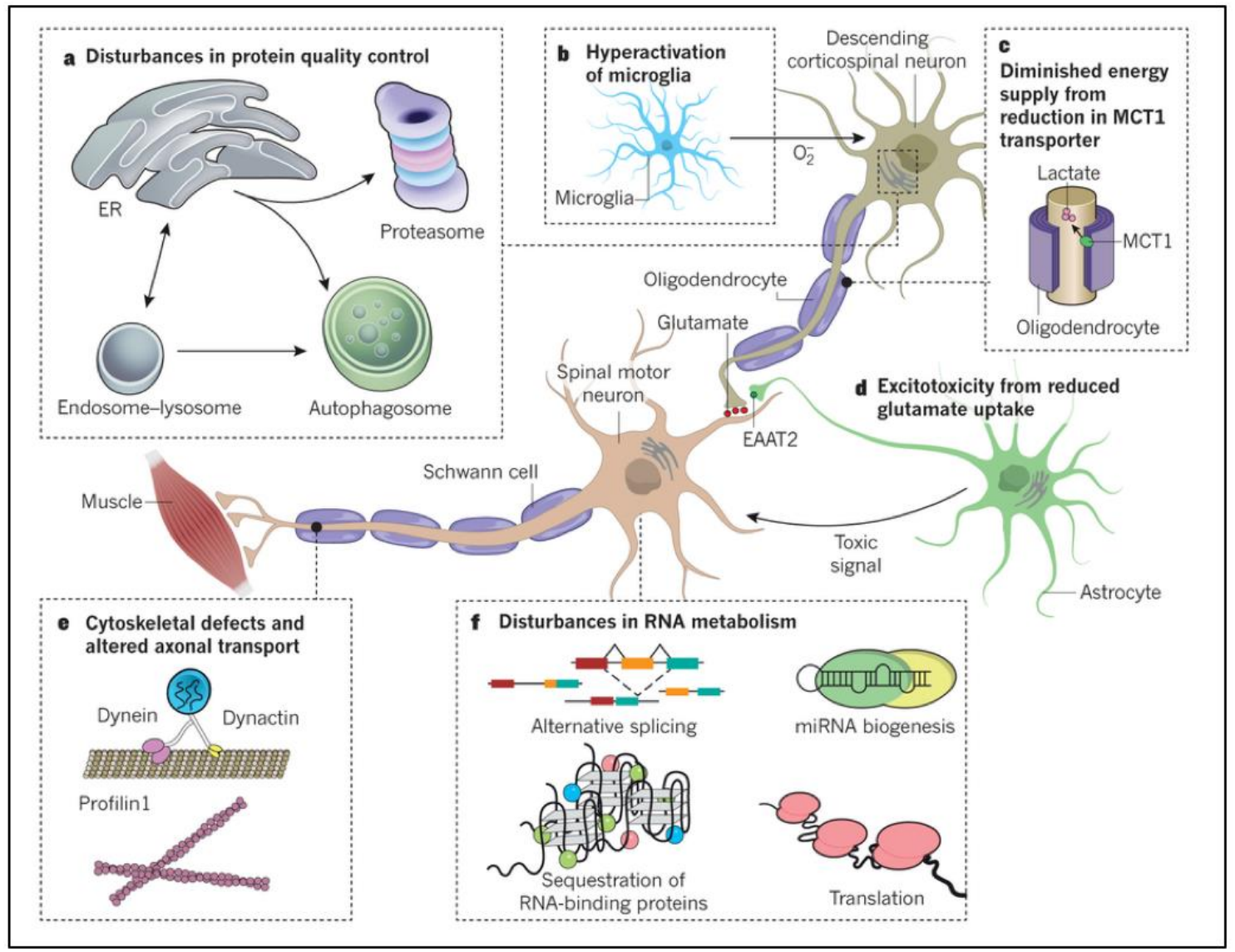

Abbildung 2 Überblick über die Pathomechanismen der ALS

Der schematische Überblick zeigt (a) den Einfluss auf das System der zellulären Protein-Qualitätskontrolle, auf (b) die hyperaktivierte Mikroglia, auf (c) die Energieversorgung der Oligodendrozyten, auf (d) das Versagen der Astrozyten in der Glutamat-Clearence des synaptischen Spalts, auf (e) den gestörten axonalen Transport und auf (f) den gestörten RNAStoffwechsel. Abbildung entnommen aus: Taylor et al. 2016, S. 201, mit freundlicher Genehmigung der Nature Publishing Group.

Eine hervorstechende Beobachtung war dabei schon früh die Entdeckung, dass bei SOD1assoziierter ALS fehlgefaltete SOD1 im Zytoplasma von Astrozyten und Motoneuronen angereichert wird und dies den Krankheitsprogress beschleunigt (Bruijn et al. 1997). Über die SOD1-Aggregate ist bekannt, dass sie an die Oberfläche des endoplasmatischen Retikulums binden und auf diese Weise zusätzlich den Abbau fehlgefalteter Proteine behindern (Saxena et al. 2009). Zudem wurde eine prionenartige Zell-zu-Zell-Ausbreitung der SOD1-Aggregate beobachtet, wie sie auch für andere neurodegenerative Erkrankungen wie Parkinson und Alzheimer postuliert wird (Münch et al. 2011, Grad et al. 2011). Bei den meisten ALS-Fällen lassen sich jedoch keine SOD1-Aggregate, sondern pTPD43Aggregate finden, für die eine solche Ausbreitung noch nicht nachgewiesen wurde, aber Teil intensiver Forschungsbemühungen ist. TDP-43 übernimmt neben anderen mit ALS assoziierten Proteinen (u. a. FUS, hnRNP A1) wichtige Funktionen im RNA-Stoffwechsel, sodass in der Störung des RNA-Stoffwechsels eine wichtige pathomechanische Auswirkung bei einer Funktionseinschränkung dieser Proteine gesehen wird (Taylor et al. 
2016). Neuroinflammation durch überaktivierte bzw. gestörte Mikroglia ist eine weitere wichtige pathomechanische Beobachtung, die sowohl bei Modellen mutierter SOD1 als auch bei Mutationen von C9ORF72 nachgewiesen wurde (Henkel et al. 2009, O'Rourke et al. 2016). Daneben wurde eine Dysorganisation des axonalen Zytoskeletts, insbesondere der Neurofilamente bei der ALS beobachtet, wodurch sowohl der anterograde (Williamson und Cleveland 1999) als auch der retrograde (Perlson et al. 2009) axonale Transport gestört sind. Als die asymmetrischsten Zellen im menschlichen Körper mit bis zu einem Meter Länge sind die Motoneurone wie keine andere Zelle auf den axonalen Transport angewiesen. Daneben ist die Exzitotoxizität durch eine zu hohe Glutamatkonzentration im synaptischen Spalt und verminderte Wiederaufnahme durch die Astrozyten ein seit vielen Jahren in der Diskussion stehender Pathomechanismus (Rothstein und Kuncl 1995, Taylor et al. 2016).

\subsubsection{Therapie und Prognose}

Bis heute gibt es keinen spezifischen Therapieansatz für die ALS, der in der Lage ist, die Erkrankung wirksam zu behandeln oder den Verlust der Neuronen aufzuhalten, gar zu stoppen. Deshalb ist die Therapie multimodal: Sie beinhaltet einerseits die Neuroprotektion und andererseits die Behandlung der zunehmenden Symptomatik, also eine palliative Therapie, die bestehend aus einem multidisziplinärem Konzept in letzter Konsequenz und nur bei ausdrücklichem Wunsch des Patienten eine invasive Beatmung umfassen kann (Leitlinie ALS 2012).

Lange galt: Einzig wirksames und oral verfügbares Pharmakon unter dutzenden bereits getesteten Wirkstoffen ist das krankheitsmodifizierende Riluzol (Handelsname: Rilutek $\left.{ }^{\circledR}\right)$ chemisch 6-(Trifluormethoxy) -1,3-benzothiazol-2-amin - in einer Dosierung von $100 \mathrm{mg}$ pro Tag. Erstmals wurde der Effekt 1994 nachgewiesen (Bensimon et al. 1994): Insgesamt konnte bislang nachgewiesen werden, dass Riluzol das mittlere Überleben um zwei bis drei Monate verlängert (Miller et al. 2012). Die Wirkung von Riluzol ist dosisabhängig (Lacomblez et al. 1996), wurde zuletzt 2013 reproduziert (Cudkowicz et al. 2013), wird umso größer, je früher mit der Therapie begonnen wird (Riviere et al. 1998) und ist insbesondere bei Patienten stark, die einen bulbären Krankheitsbeginn aufweisen (Miller et al. 2012). Seit 2017 ist mit Edaravone noch ein weiteres wirksames Pharmakon verfügbar (Rothstein 2017).

Mit der Erprobung von Riluzol in den 90er Jahren im Rahmen klinischer Studien zu ALS ging die Vermutung einher, eine chronische Aktivierung von Glutamat-Rezeptoren und eine damit einhergehende Exzitotoxizität sei eine Ursache von Neurodegeneration und 
mitverantwortlich für die Entstehung von ALS (Rothstein 1996). Die neuroprotektive Wirkung von Riluzol war zuvor bereits in verschiedenen präklinischen Studien und anderen Krankheitsmodellen nachgewiesen worden (Pratt et al. 1992, Doble et al. 1992, Hubert et al. 1994, Herbert et al. 1994, Rothstein und Kuncl 1995). Riluzol als Wirkstoff, der die Konzentration von Glutamat im synaptischen Spalt reduziert, indem die präsynaptische Freisetzung gehemmt und die Aufnahme von Glutamat durch Astrozyten und Neurone erhöht wird, und so das Überleben der Patienten verlängert, scheint die These der Glutamat-Toxizität bzw. Exzitoxizität noch weiter zu unterstützen. Jedoch ist die Wirksamkeit offensichtlich sehr begrenzt und aufgrund von Kritik an den Einschlusskriterien für die klinischen Studien, in denen Riluzol bisher getestet wurde, nicht unumstritten und es bedarf weiterer Studien zu den Fragen, inwieweit Riluzol einen Einfluss auf die Lebensqualität und respiratorische Funktion hat, sowie, ob es sich auch bei alten oder bereits stark betroffenen Patienten als wirksam auszeichnen kann (Miller et al. 2012). Deshalb und aufgrund der vor allem im individuellen Fall sehr begrenzten Wirksamkeit ist der Bedarf für die Entwicklung einer neuen oder ergänzenden pharmakologischen Therapie für die ALS immer noch sehr groß.

Wegen der infausten Prognose der Patienten und der immer weiter fortschreitenden Symptomatik ist eine multidisziplinäre symptomatische Therapie von zentraler Bedeutung für die stationäre und ambulante Behandlung von ALS-Patienten. Dazu gehört Krankengymnastik, Ergotherapie, Atemtherapie, Pneumonie-Prophylaxe, Thromboseprophylaxe, eine hochkalorische Diät, Behandlung der Schluckstörung - ggf mit Einsatz einer PEG-Sonde, Psychotherapie und Psychopharmakotherapie, Anxiolyse, Schmerztherapie, Behandlung der Muskelkrämpfe, Spasmolyse, eine optimale Ausstattung mit Hilfs- und Heilmitteln, eine nicht-invasive Beatmung bereits in frühen Phasen der Erkrankung und in letzter Konsequenz und bei ausdrücklichem Wunsch des Patienten auch eine invasive Beatmung (Leitlinie ALS 2012).

Die ALS verläuft immer progredient und ist nicht heilbar, die Prognose ist infaust bei einem mittleren Überleben von circa zwei bis drei Jahren bei einem bulbären Krankheitsbeginn und drei bis fünf Jahren bei einem Krankheitsbeginn in den Extremitäten, die 5-Jahres-Überlebensrate wird mit Werten zwischen 48\% und 24\% angegeben (Wijesekera und Leigh 2009), nur 5-10\% der Patienten überleben länger als 10 Jahre (Chio et al. 2009). Faktoren, die prognostisch günstig sind und einen langsameren Krankheitsverlauf bedingen können, sind das Erkranken vor dem 40. Lebensjahr mit einem drei- bis viermal längerem Überleben - es konnte insgesamt ein umgekehrt exponentieller Zusammenhang zwischen Erkrankungsalter und Krankheitsdauer beobachtet werden 
(Eisen et al. 1993) - und ein vorwiegender Befall des ersten Motoneurons (Sorarù et al. 2010, Sabatelli et al. 2011). Negative prognostische Faktoren sind ein bulbärer oder ein respiratorischer Symptombeginn, ein niedriger BMI und ein niedrige respiratorische Vitalkapazität bei Krankheitsbeginn, eine bei erster Diagnosestellung nach den El-EscorialKriterien sichere ALS und laut einigen Studien ein kurzer Zeitraum von Symptombeginn bis Diagnosestellung (del Aguila et al. 2003). Außerdem nehmen psychosoziale und kognitive Faktoren vermutlich Einfluss auf den Krankheitsverlauf, nicht zuletzt durch deren Einfluss auf die Akzeptanz des Patienten und seines Umfeldes von lebensverlängernden Maßnahmen wie nicht-invasive bzw. invasive Beatmung oder künstliche Ernährung durch eine PEG-Sonde (Chiò et al. 2009).

\subsection{Das High-copy-B6SJL-Tg(SOD1-G93A)1Gur/J-Mausmodell}

\subsubsection{Genetischer Hintergrund und Geschichte}

Das transgene Mausmodell mit der Punktmutation G93A auf dem für SOD 1 kodierenden Genabschnitt ist das am häufigsten verwendete Modell für die ALS in präklinischen Studien zur Testung von pharmakologischen Therapien (Andersen 2006). Das Metalloenzym ist das erste von drei Proteinen aus der Gruppe der Superoxiddismutasen (SOD): die Kupfer und Zink enthaltende Superoxiddismutase (SOD1). Das SOD1G93Atransgene Mausmodell ist eines der ersten entwickelten Tiermodelle, das zuverlässig und reproduzierbar für die präklinische Erforschung einer Erkrankung genutzt wird, deren therapeutische Behandlung zu diesem Zeitpunkt in den Kinderschuhen steckte (Lacomblez et al. 1996, Ludolph et al. 2010). Die Entwicklung von Therapeutika im Bereich der ALS basiert weitestgehend auf Tierexperimenten mit diesem transgenen Tierstamm, der mutierte humane SOD 1 trägt (Ludolph 2006).

Transgene Mausmodelle mit überexprimierter humaner SOD1 vom Wildtyp, die auf Chromosom 21 kodiert ist, waren bereits Ende der 1980er Jahre als Modell für das DownSyndrom entwickelt worden, um die spezifischen Auswirkungen der erhöhten $\mathrm{Cu} / \mathrm{Zn}$ SOD im Down Syndrom und anderen Prozessen zu erforschen (Epstein et al. 1987). Es wurden bei diesen Mäusen in der Folge neben verschiedenen klinischen und laborchemischen Veränderungen wie zum Beispiel einer erniedrigten peripheren Serotoninausschüttung und Prostaglandin-Synthese (Schickler et al. 1989, Minc-Golomb et al. 1991, Turner und Talbot 2008) auch muskuläre Pathologien in den Hinterläufen gefunden (Avraham et al. 1988, Avraham et al. 1991) sowie der Verlust von Axonen und 
die Degeneration von Motoneuronen im Rückenmark bei gealterten Tieren (Dal Canto und Gurney 1995).

Als Anfang der 1990er Jahre verschiedene Mutationen der SOD1 als Auslöser der fALS erkannt wurden (Rosen et al. 1993), folgte prompt die Entwicklung transgener Mausstämme mit mutierter SOD1 (Gurney et al. 1994, Turner und Talbot 2008).

Die SOD1 besteht aus 153 Aminosäuren und macht immerhin ungefähr 0,5\% des Gesamtproteingehalts des menschlichen Gehirns aus. Es wurden seit der Entdeckung ihrer Bedeutung für die fALS bereits 108 Missense-Muationen und 11 Nonsense-Mutationen im codierenden Genabschnitt gefunden, die mit der ALS assoziiert werden können (Andersen 2006). Durch viele dieser Mutationen verliert das Enzym allerding nicht seine ursprüngliche Funktion, sondern man geht davon aus, dass es toxische Eigenschaften (gain of funtion) hinzugewinnt (Borchelt et al. 1994, Borchelt et al. 1995).

Das Mausmodell mit einer hohen Anzahl von Genkopien - 18 Kopien pro diploidem Genom - der auf dem Exon 4, Codon 93 von Glycin zu Alanin mutierten SOD1 zeigte sich als in der klinischen Symptomatik der ALS sehr ähnlich und sehr stabil im klinischen Verlauf (Turner und Talbot 2008). Die Firma Jackson Labs vertreibt auf Grundlage dieser G1-Linie genannten Linie, die ursprünglich von Mark E. Gurney entwickelt wurde (Gurney et al. 1994), eine Mauslinie mit nochmals auf 25 erhöhter Anzahl von Genkopien - die G1H bzw. 1Gur-Linie (Tu et al. 1996).

\subsubsection{Phänotyp: Klinischer Verlauf und Pathophysiologie}

Die Mäuse entwickeln bereits im Alter von ca. drei Monaten die ersten klinischen Anzeichen für eine Erkrankung des motorischen Systems. Die Symptome beginnen mit einem feinschlägigen Tremor in einer oder mehrerer Extremitäten beginnend i.d.R. um den 91. Lebenstag. In den folgenden Wochen ist ein Ausbreiten des Tremors zu beobachten sowie eine zunehmende Hyperreflexie und Spastik: Bei passiver Bewegung der hinteren Extremität bei Mäusen, die bereits einen Tremor haben, zeigt sich ein erhöhter Widerstand als erster Ausdruck der Spastik; bei leichtem Beklopfen der Knie oder der Knöchel zeigt sich eine starke Reflexantwort, sogar ein Überspringen auf die Gegenseite kann beobachtet werden. In der Folge kommt es dann zu einer Verlangsamung des Wachstums und der normalen Gewichtszunahme, die auf einem Plateau, das dem Normalgewicht von 75 Tage alten nicht-transgenen Mäusen entspricht, stagniert. Es kommt zu einer stetigen Zunahme von Muskelschwäche und Atrophie mit Betonung der hinteren Extremitäten bis hin zur Unfähigkeit, das Becken vom Boden anzuheben, sodass die Fortbewegung nur noch durch Hinterherschleifen der beiden hinteren Extremitäten durch die vorderen möglich ist. 
Letztendlich sind die Mäuse schwer gelähmt und liegen auf der Seite, unfähig sich selbst aufzurichten. Anhand von Tierversuchsabbruchkriterien werden die Tiere diesen entsprechend eingeschläfert und haben eine durchschnittliche Lebenserwartung von 128 bis 143 Tagen (Chiu et al. 1995).

Die Pathophysiologie hinter dieser fatalen klinischen Entwicklung wurde eingehend untersucht, und bereits in frühen Lebensphasen wurden Veränderungen festgestellt. Die muskulären Endplatten degenerieren bereits um den 47. Lebenstag (Fischer et al. 2004), noch früher ist eine periphere Denervierung ab dem 25. Lebenstag nachzuweisen (Gould et al. 2006), während ein proximaler Axonverlust, sowie ein Verlust der unteren Motoneurone im Rückenmark erst zwischen dem 80. und 100. Lebenstag beobachtet werden kann. Diese Entdeckung führte zu der Vermutung, die ALS könne möglicherweise eine retrograde Neurodegeneration im Sinne einer Axonopathie sein (Fischer et al. 2004). Jedoch können auch in den spinalen Motoneuronen Pathologien sehr früh nachgewiesen werden: eine mitochondriale Vakuolation (Dal Canto und Gurney 1995) und eine Fragmentierung des Golgi-Apparates (Mourelatos et al. 1996) wurden in diesen nachgewiesen. Ebenfalls wurden im Rückenmark eine Astrozytose und Mikrogliose beschrieben (Hall et al. 1998), und der Nachweis von SOD1-immunoreaktiven Aggregaten gelang (Johnston et al. 2000). Nicht nur im Rückenmark konnte die Degeneration von Motoneuronen nachgewiesen werden, auch in den die entsprechenden Hirnnerven versorgenden Kerngebieten der Nn. trigemini, Nn. faciales und Nn. hypoglossi konnte eine Degeneration nachgewiesen werden (Zang et al. 2004).

\subsubsection{Verhaltensbiologische Methoden der Krankheitsbeobachtung}

In der vorliegenden Studie wurden etablierte verhaltensbiologische Methoden der Krankheitsbeobachtung des SOD1G93A-Mausmodells angewandt wie die Beobachtung des Überlebens, des Gewichts, des klinisch-neurologischen Erscheinungsbild mithilfe des klinisch-neurologischen Scores nach Weydt et al. (2003) und der Beobachtung der motorischen Performance mittels des motorkoordinativen Drehwalzenversuchs (Rotarod). Es erscheint aber lohnenswert einerseits mit noch genaueren Methoden die motorische Performance, aber andererseits auch im Hinblick auf die Erkenntnisse in Bezug auf die Verwandtschaft von ALS und FTLD die kognitive Entwicklung im SOD1G93AMausmodell zu beobachten.

\subsubsection{Kognitive Bewertung}

Bisher haben sich nur wenige Studien mit dem kognitiven Verhalten des in der vorliegenden Studie verwendeten Mausmodells beschäftigt. Über den gewählten 
Hintergrund einer 1. Generation (F1) weiblicher Mäuse von C57BJ-Mäusen, gepaart mit SJL-Mäusen (F1C57BJxSJL), deren Eigenschaften auch den Wildtyp-Wurfgeschwistern des gewählten transgenen Mausmodells unterstellt werden dürfen (Spalloni et al. 2006), ist bekannt, dass sie ein signifikant geringeres Explorationsverhalten an den Tag legen als andere Mausstämme (Dellu et al. 2000). Bedingt ist dies womöglich durch einen Gendefekt, der eine Degeneration der Retina bei Mäusen des SJL-Stamms hervorruft. Dieses Defizit könnte auch die Mäuse der Kreuzung mit C57BJ betreffen. Spalloni et al. (2006) konnten bei männlichen transgenen Mäusen des SOD1G93A-Mausmodells zwischen Tag 90 und 100 ein höheres Explorationsverhalten im Vergleich zu ihren gesunden WildtypGeschwistern nachweisen. So scheint bei den transgenen Tieren dieses Modells die besondere Eigenschaft des geringen Explorationsverhaltens im Hintergrund-Stamm nicht oder weniger vorhanden $\mathrm{zu}$ sein. Diese grundlegenden Beobachtungen zum Explorationsverhalten der Wildtyp-Tiere und der transgenen Tiere müssen bei der Interpretation der Ergebnisse einer kognitiven Untersuchung im SOD1G93A-Mausmodell beachtet werden.

\subsubsection{Ganganalyse}

Da das SOD1G93A-Mausmodell starken motorischen Veränderungen ausgesetzt ist, ist die Ganganalyse mehrfach in der Vergangenheit im Fokus der Wissenschaft gewesen, um mit einer solchen Analyse die Symptomatik der Erkrankung noch besser zu beobachten und objektivieren zu können. Wooley et al. (2005) konnten bei einem SOD1G93AInzuchtstamm mit C57/B6J-Hintergrund, dessen Verwendung bei der Zucht einen weniger rapiden Krankheitsverlauf zu Folge hat, bereits im Alter von 8 und 10 Wochen Veränderungen in den untersuchten Gangparametern feststellen: Mithilfe einer selbst konzipierten videobasierten Ganganalyse auf einem Laufband mit einer fest einstellbaren Geschwindigkeit konnten längere Steh- und Schrittzeiten im Vergleich zur gesunden Kontrollgruppe beobachtet werden. Die Hinterläufe waren dabei einer stärkeren Verschlechterung unterworfen. Einen ähnlichen Versuchsaufbau nutzten Guillot et al. (2008), um an drei weiblichen und drei männlichen SOD1G93A-transgenen Tieren, ebenfalls mit reinem C57/B6J-Hintergrund, den Gang mit der DigiGait-Software (Mouse Specifics, Inc., Boston, Massachuesetts, Vereinigte Staaten) auszuwerten. Dabei konnten die zuvor gewonnenen Erkenntnisse über eine Verschlechterung der Gangparameter von Wooley et al. (2005) nicht reproduziert werden.

Knippenberg et al. (2010) untersuchten Gangveränderungen im SOD1G93A-Mausmodell ab der 11. Lebenswoche einmal wöchentlich mit einer Fußabdruckanalyse, bei der die Mäuse, nachdem ihre Hinterläufe mit Tinte benetzt wurden, auf einem $50 \mathrm{~cm}$ langem, $5 \mathrm{~cm}$ 
breiten und auf beiden Längsseiten begrenzten Papierstreifen entlanglaufen. Dabei wurden einerseits die Zeit eines Laufes und später mit einer Bildanalyse-Software die Schrittlänge der Hinterläufe ausgewertet. Es zeigten sich ab der 18. Lebenswoche eine signifikante Verkürzung der Schrittlänge und eine signifikante Verlängerung der Zeit eines Laufes der SOD1G93A-Mäuse im Gegensatz zu den SOD1Wt-Mäusen. Die Methode der Fußabdruckanalyse mittels Tintenabdruck wird von Mancuso et al. (2011), die sie im Alter von 8, 12 und 16 Wochen ebenfalls bei Tieren des SOD1G93A-Mausmodells durchführten und dabei keine Unterschiede in der Schrittlänge fanden, allerdings kritisiert, da die Mäuse normalerweise keinen durchgehenden Lauf über $50 \mathrm{~cm}$ freiwillig durchführen würden. Mancuso et al. (2011) nutzten daher eine videobasierte Ganganalyse (DigiGait, Mouse Specifics, Inc., Boston, Massachuesetts, Vereinigte Staaten) auf einem Laufband mit festeinstellbarer Geschwindigkeit an den gleichen drei Zeitpunkten. Dabei untersuchten sie neben Auftrittsbreite und Fußstellung vor allem zeitliche Parameter einzelner Schrittphasen. Sie konnten bei den transgenen Tieren in der 8. Woche eine signifikante Verschlechterung von zwei der sieben ausgewerteten Parameter beobachten, in der 12 . Woche bereits fünf, in der 16. Woche waren alle sieben Parameter signifikant schlechter.

Mehrere Autoren untersuchten das SOD1G93A-Mausmodell wie die vorliegende Studie mit dem CatWalk XT gait analysis system (Noldus, Wageningen, Niederlande): Dabei konnten Mead et al. (2011), die wöchentliche Analysen an fünf SOD1G93A-transgenen Mäuse beginnend am Lebenstag 35 bis zum Lebenstag 105 durchführten, Defizite in der Auftrittszeit (stand) an mehreren Untersuchungszeitpunkten, und vor allem eine Verkürzung der Schrittlänge der Hinter- und Vorderläufe (stride lengtb) bei fortschreitender Erkrankung feststellen. Zudem konnten eine Abnahme des normalen diagonalen Auftritts (support diagonal) und eine Zunahme der Zuhilfenahme einer dritten Extremität (support three limbs) im Gang bei den transgenen Tieren festgestellt werden.

Gerber et al. (2012) führten die CatWalk-Ganganalyse beim SOD1G93A-Mausmodell ab dem Lebenstag 50 wöchentlich durch und konnten in ihrer Auswertung der relativen Position des Hinterlaufs zum Vorderlauf - gesunde Kontrolltiere setzen den seitengleichen Hinterlauf im nächsten Schrittzyklus fast genau an die zuvor vom Vorderlauf besetzte Position - bereits ab dem Lebenstag 60 signifikante lokomotorische Defizite transgener Tiere nachweisen, die sich im Krankheitsverlauf noch verstärkten.

Vergouts et al. (2015) konnten in ihrer CatWalk-Ganganalyse von 10 weiblichen SOD1G93A-transgenen Mäusen im Alter von 16 Wochen in allen ausgewerteten Gangparametern Defizite der Hinter- und Vorderläufe finden, u. a. Schrittlänge (stride 
length) Auftrittszeit (stance duration), Schrittzeit (swing duration), Schrittgeschwindigkeit (swing speed) und relative Position (relative position).

\subsection{ROCK-Inhibition}

\subsubsection{ROCK1 und ROCK2: Rho-assoziierte Coiled-Coil containing Proteinkinasen}

Die beiden Rho-assoziierten Coiled-Coil containing Proteinkinasen (ROCK)1 (auch ROK $\beta$ ) und ROCK2 (auch ROK $\alpha$ ) sind Serin-Threonin-Proteinkinasen aus der Familie der AGC-Kinasen, die aus mehr als 50 verschiedenen Proteinen besteht (Proud 2007), werden von RhoA, einer Rho-GTPase, aktiviert und haben eine Vielzahl an Effektormolekülen. Sie bestehen aus drei Hauptdomänen: der Rho-bindenen Domäne in der Coiled-Coil-Region, der Kinase-Domäne, die für die katalytische Aktivität des Proteins verantwortlich ist, und eine Pleckstrin-homologe Domäne mit einer cysteinreichen Region, die für die Regulation und die Lokalisation des Proteins in der Zelle verantwortlich ist (Schmandke et al. 2007).

ROCK1 und ROCK2 teilen sich mit $65 \%$ einen Großteil ihrer Aminosäuresequenz, insbesondere in der katalytischen Domäne stimmen sie überein - zu 92\% (Nakagawa et al. 1996). Trotz dieser weitreichend strukturellen Gemeinsamkeiten unterscheiden sie sich deutlich in ihrem Vorkommen im Organismus: Während ROCK2 vor allem im Herz und im Gehirn vorkommt, kommt ROCK1 vor allem in nicht-neuronalen Geweben vor. Eine besonders hohe Expression von ROCK2 weisen die Pyramidenzellen des Hippocampus und des zerebralen Kortex sowie die Purkinjezellen des Zerebellums auf (Hashimoto et al. 1999).

ROCK1 und ROCK2 werden von der Rho-GTPase RhoA aktiviert und liegen normalerweise als eine sich selbst inhibierende Einheit vor, erst durch die Bindung der aktivierten RhoA an der Rho-bindenden Domäne wird die katalytische Einheit aktiviert. Inhibitoren von ROCK sind Gem und Rad, die als Rad-Gem-Kir-Familie (RGK) Teil der Superfamilie der Ras-zugehörigen kleinen GTPasen sind, indem sie an der Coiled-Coil Domäne binden (Ward et al. 2002). Eine andere kleine GTPase, RhoE, wurde als ROCK1Inhibotor beschrieben, indem es an die Kinase-Domäne bindet (Riento und Ridley 2003). Rho-GTPasen sind regulatorische Proteine und gehören ebenfalls in die Superfamilie der Ras-zugehörigen kleinen GTPasen und sind somit eine Art G-Protein, die im Zytosol zu finden sind. Sie sind eine von 9 Untergruppen der Superfamilie und werden selbst in 8 Untergruppen geteilt (Blumenstein 2004, Schmandke et al. 2007). Ungefähr ein Prozent des gesamten menschlichen Genoms kodiert Proteine, die entweder Rho-GTPasen regulieren 
oder durch diese reguliert werden. Rho-GTPasen wechseln zwischen einem inaktiven GDP-gebundenem und einem aktiven GTP-gebundenen Zustand. In allen eukaryotischen Zellen regulieren auf diese Weise Rho-GTPasen unter anderem: Morphogenese, Polarität, Zellbewegung und Zellteilung (Jaffe und Hall 2005). Rho-GDP wird durch Guanin nucleotid exchange factors (GEFs) in Rho-GTP umgewandelt und so aktiviert. Andererseits wird diese Umwandlung von Guanine nucelotid dissociation inbibitors (GDIs) inhibiert und GTPase activating proteins (GAPs) führen zu einer Rückumwandlung durch eine erhöhte intrinsische GTPase-Aktivität. Die Aktivierung von RhoA wird über verschiedene Signalwege vermittelt: Über Rezeptor-Tyrosinkinasen (Schiller 2006), über G-Protein gekoppelte Rezeptoren (GPCRs) und über andere Transmembranproteine wie z. B. Plexine oder den Nogo-Rezeptorkomplex (Schmandke et al. 2007). Neben RhoA sind Arachidonsäure und Sphingosylphosphorylcholin unspezifische intrazelluläre Aktivatoren von ROCK, während Caspase-3 im Rahmen der Apoptose den autoinhibitorischen C-Terminus proteolytisch spalten kann und so zu einer erhöhten ROCK1-Aktivität führt (Schmandke et al. 2007, Jacobs et al. 2006, Coleman et al. 2001).

Es wurden bereits eine Menge Effektorproteine von ROCK1 und ROCK2 beschrieben. Neben der Modulation des neurotrophen PI3/Akt-Signalwegs (Wolfrum et al. 2004) und des Insulin-Signalwegs (Farah et al. 1998) stehen im Bereich der Neurodegenerativen Erkrankungen auch Proteine im Fokus der aktuellen Forschung, die für die Regulation des Aktin-Zytoskeletts und der myosinbasierten Zellkontraktilität verantwortlich sind. Einerseits führt eine erhöhte ROCK-Aktivität $\mathrm{zu}$ einer erhöhten myosinbasierten Zellkontraktilität und andererseits zu einer erniedrigten Reorganisation und reduziertem Aufbau des Aktinzytoskeletts:

Als besonders gut studiertes Effektorprotein gilt die Myosin-Leichte-Ketten-Phosphatase (MLCP), deren myosin binding site (MBS) von ROCK phosphoryliert wird und deren Aktivität dadurch gesenkt wird (Kawano et al. 1999). Die geringere Aktivität der MLCP führt zu einer geringeren Dephosphorylierungsrate - also niedrigeren Inaktivierung - der myosin-II regulatory light chain (MLC). Zudem kann ROCK2 auch die myosin light chain kinase (MLCK) und auch die MLC selber phosphorylieren, um diese zu aktivieren. Daneben gibt es auch die Möglichkeit, dass RhoA an die MBS bindet und somit einen ROCKunabhängigen Weg, die myosinbasierte Kontraktilität der Zelle zu regulieren (Kimura et al. 1996).

Durch die Phosphorylierung von LIM-Kinase (LIMK), welche durch diese Aktivierung ihrerseits Cofilin phophoryliert und damit inhibiert (Sarmiere und Bamburg 2004), und durch die Inhibierung von Profilin führt eine erhöhte ROCK-Aktivität zu einer 
erniedrigten Aktin-Depolymerationsrate und einer reduzierten Reorganisation des Aktinzytoskeletts (Maekawa et al. 1999), was zu einem reduzierten axonalen Wachstum beiträgt (Ng und Luo 2004).

Daneben sind $\alpha$-Adducin (Kimura et al. 1998) und die ERM-Proteine Etrin, Radixin und Meosin weitere Effektoren von ROCK, die das Aktinzytoskelett beeinflussen (Matsui et al. 1998). Und auch die Intermediärfilamentproteine Vimentin, das saure Gliafaserprotein (GFAP) und das Neurofilamentprotein (NF-L) werden von ROCK2 phosphoryliert (Schmandke et al. 2007).

\subsubsection{Rolle von ROCK bei Neurodegeneration}

Eine erhöhte ROCK-Aktivität wird seit einiger Zeit in Zusammenhang mit den Pathomechanismen neurodegenerativer Erkrankungen gesehen und ein möglicher vorteilhafter Effekt von ROCK-Inhibition wurde in vielen Modellen in vivo und in vitro untersucht (siehe Kapitel 1.3.4). Die erhöhte ROCK-Aktivität wird dabei im Rahmen des Signalweges und im Rahmen des Zelltyps gesehen (Hensel et al. 2015) und hat vermutlich zellschädigende Auswirkungen.

Wie bereits in Kapitel 1.3.1 beschrieben, wirkt eine erhöhte ROCK-Aktivität im Rahmen anerkannter Signalwege inhibierend auf MLCP, Cofilin und Profilin, was einerseits zu einer erhöhten myosinvermittelten Zellkontraktilität und anderseits zu einer verminderten Reorganisation und Wachstum des Aktin-Zytoskeletts führt. In neuronalen Zellen führt das zu einem Kollaps des Wachstumskegels, was zu der Annahme führt, dass ROCK eine schädigende Wirkung auf Regeneration und neuronales Wachstum hat. Hinzu kommt, dass ROCK neurotrophe Signalwege, die phosphatase and tensin homolog (PTEN), Akt und extracellular signal-regulated kinase (ERK) beinhalten, welche als Agonisten neuronalen Überlebens gelten, negativ kontrolliert. Zwar sind die genauen molekularen Mechanismen dabei unbekannt, jedoch kann angenommen werden, dass eine erhöhte ROCK-Aktivität über diesen Weg zu einem verminderten neuronalen Überleben führt (Lingor et al. 2008, Takata et al. 2013).

Zudem führt eine erhöhte ROCK-Aktivität im Rahmen der durch die neuronale Schädigung stattfindenden Neuro-Inflammation dazu, dass Mikrogliazellen im zellschädigendem M1-Zustand verweilen und nicht in den antientzündlichen, Gewebe unterstützenden und die Extrazellulär-Matrix reparierenden M2-Zustand übergehen (Zhang et al. 2013).

Die beschriebenen molekularen und zellulären Mechanismen, die für alle neurodegenerativen Erkrankungen gelten können, legen nahe, dass sich eine ROCK- 
Inhibition vorteilhaft auf unterschiedliche neurodegenerative Erkrankungen auswirken kann. Die möglichen Effekte sollten entsprechend unter den folgenden pathomechanischen Gesichtspunkten gesehen werden: Unterstützung des axonalen Wachstums, Unterstützung des neuronalen Überlebens und Inhibition deletärer NeuroInflammation (Hensel et al. 2015).

\subsubsection{Pharmakologische Inhibition von ROCK}

Die beschriebenen Effektorproteine von ROCK sind Schlüsselproteine in neuronalen Prozessen wie Axogenese, Wachstumskegeldynamik (growth cone dynamics) und der Stabilität von Synapsen (Hensel et al. 2015).

Eine erhöhte ROCK-Aktivität wirkt schädlich bei neurodegenerativen Erkrankungen, was bereits in verschiedenen In-vivo- und In-vitro-Modellen gezeigt wurde (siehe Kapitel 1.3.4). Dabei wurde häufig der potente ROCK2-Inhibitor Y-27632 genutzt. Jedoch gibt es mit Fasudil bereits einen anderen ROCK-Inhibitor, der schon seit 1995 für die Behandlung beim Menschen zugelassen ist und ROCK1 und ROCK2 gleichermaßen hemmt.

Fasudil, auch (HA1077) 1- (5-isoquinolinesulphonyl) homopiperazine), aus der Gruppe der Sulfonamide ist ein Proteinkinase-Inhibitor (Asano et al. 1989) mit Wirkung auf mehrere Proteinkinasen. Anfangs wurde vor allem die vasodilatatorische Wirkung Fasudils im Rahmen der zerebralen Vasospasmen nach Subarachnoidalblutung erforscht und seine Überlegenheit nachgewiesen (Takayasu et al. 1986). Seine vasodilatatorische Wirkung erreicht Fasudil dabei im Gegensatz zu bis dato zugelassenen Präparaten und zusätzlich zu der Hemmung der über die MLC bewirkten Kontraktion auch durch eine Reorganisation der Struktur der Aktin-Mikrofilamente in den glatten Muskelzellen (Sasaki et al. 1987). Die Wirkung auf diese von der Rho-ROCK-Signalkaskade gesteuerten Prozesse erreicht Fasudil als potenter ROCK1- und ROCK2-Inhibitor. Daneben hemmt Fasudil auch Proteinkinase C, Proteinkinase N, Proteinkinase A, MAP-Kinase 1 und MLCK (Lingor et al. 2007, Nagumo et al. 2000, Davies et al. 2000). Durch die bereits erwiesene Applikation beim Menschen und Zulassung für die Behandlung bei Schlaganfallpatienten in Japan seit 1995 ist Fasudil auch im Bereich Neurodegeneration ein vielversprechendes Präparat (Chen et al. 2013).

\subsubsection{ROCK-Inhibition in Modellen der Neurodegeneration - ein Überblick}

Molekulare und zelluläre Mechanismen im Rahmen neurodegenerativer Erkrankungen legen eine neuroprotektive Wirkung von ROCK-Inhibition und eine schädigende Wirkung erhöhter ROCK-Aktivität nahe. Diese Annahme wurde in In-vivo- und In-vitro-Modellen demonstriert, von denen hier einige erwähnt seien. 
Grundlegend günstige Effekte auf Neurodegeneration und Neuroregeneration wurden von Lingor et al. (2007) mit den ROCK-Inhibitoren Y-27632, Fasudil und Dimethylfasudil (H1152) in vivo und in vitro nachgewiesen: Das Neuriten-Wachstum in einer Zellkultur von Ntera-2-Neuronen wurde durch die Behandlung dosisabhängig gesteigert und in einem Invivo-Modell für Neurodegeneration wurde eine verbesserte Neuroregeneration beobachtet. Im Bereich des Morbus Parkinson/Parkinson's Disease (PD) wurde im Rahmen Toxininduzierter Modelle in vitro und in vivo eine Verbesserung unter der Behandlung mit Fasudil beobachtet: Im MPTP (1-methyl-4-phenyl-1,2,3,6-tetrahydropyridine) -Mausmodell wurde eine weniger stark verminderte Zellzahl dopaminerger Neurone in der Substantia nigra und eine verbesserte motorische Performance beobachtet. In der Zellkultur dopaminerger Mittelhirn-Neurone der Maus, die mit MPP+ (1-methyl-4-phenylpyridinium) behandelt wurden, zeigten die mit optimaler Konzentration Fasudil $(20 \mu \mathrm{M})$ behandelten Kulturen eine erhöhte Aktivität des auf das neuronale Überleben vorteilhaft wirkenden AktSignalwegs, eine höhere Zahl an TH (tyrosine hydroxylase) positiven Zellen und eine erhöhte Neuriten-Länge (Tönges et al. 2012). Die Annahme einer gegenseitigen Beeinflussung des ROCK-Signalwegs und der anti-apoptotischen Signalwege von ERK und Akt wird zudem durch die Beobachtungen an einem AAV2-mediated knockdown-Modell von ROCK2 beobachtet. In vitro kann dort eine erhöhte ERK-Aktivität, eine weniger starke Abnahme TH-positiver Zellen und in vivo eine milde Verbesserung der motorischen Performance beobachtet werden (Saal et al. 2015).

Im Bereich der Multiplen Sklerose (MS) wurde eine erhöhte ROCK-Aktivität im zentralen Nervensystem bei Mäusen eines mit proteolipid protein (PLP) induzierten experimental autoimmune encephalomyelitis (EAE)-Modells gefunden, während gleichzeitig beobachtet wurde, dass Fasudil die induzierte EAE unterdrückt, indem es die Infiltration des ZNS durch Entzündungszellen hemmt (Sun et al. 2006). Da bei der chronisch verlaufenden MS eine lange Applikationsdauer erwartet werden kann, wurde in der Folge nach alternativen Applikationsformen, die zielgerichteter sind, gesucht. Im Rahmen einer Studie mit einem murinen myelin oligodendrocyte glycoprotein (MOG)-induzierten EAE-Modell wurde unter intranasaler Verabreichung eines Fasudil-Derivates eine reduzierte Demyelinisierung, verbesserte klinische Parameter und ein erhöhtes Körpergewicht beobachtet ( $\mathrm{Li}$ et al. 2014).

Im Bereich des Morbus Alzheimer/Alzeimer's Disease (AD) wurde in den Gehirnen von Patienten mit idiopathischer AD ein erhöhtes Protein-Level von ROCK2 gefunden. Diese Beobachtung konnte auch bei Patienten mit mild cognitive impairment (MCI), einer als Vorstufe der AD verstandenen Erkrankung, bestätigt werden (Herskowitz et al. 2013). In 
einem transgenen Mausmodell für AD (PDAPP-Mausmodel) zeigte sich, dass unter Behandlung durch intrazerebroventrikuläre Injektion des ROCK-Inhibitors Y-27632 signifikant weniger Amyloid- $\beta$ im Gehirn der Tiere vorhanden ist (Zhou et al. 2003). Einen deutlichen Unterschied von ROCK1 zu ROCK2 konnte in der Beeinflussung des Amyloid$\beta$-Stoffwechsel festgestellt werden: Während ein ROCK1 knock-down die Amyloid- $\beta$ Produktion erhöht, reduziert die spezifische ROCK2-Inhibition durch SR3677 Amyloid- $\beta$ in vivo und in vitro (Herskowitz et al. 2013). Daraus lässt sich schließen, dass im Bereich der AD eine spezifische ROCK2-Inhibition das vielversprechendere Target ist, weil eine ROCK1-Inhibition möglicherweise einen schädigenden Einfluss haben könnte (Hensel et al. 2015).

Im Bereich der Chorea Huntington/Huntington's Disease (HD), bei der die durch eine CAGWiederholungsmutation im Huntingtin(Htt)-Gen Oligomere und Aggregate von $\mathrm{Htt}$ in Neuronen eine schädigende Wirkung haben, wurde beobachtet, dass ROCK-Inhibition mit Y-27632 den Abbau der Aggregate in vitro fördert (Bauer et al. 2009). Jedoch konnte in einem transgenen Mausmodell für die HD, dem R6/2 HD-Mausmodell, kein Einfluss auf deren Überleben, die Zellatrophie oder das Aggregationsverhalten in den Zellen neben einer nur milden Verbesserung der motorischen Performance beobachtet werden ( $\mathrm{Li}$ et al. 2009). In einer anderen Studie konnte allerdings ein verbessertes neuronales Überleben und eine verbesserte axonale Regeneration unter ROCK-Inhibtion in einem In-vitro-HD-Modell gezeigt werden (Deyts et al. 2009), sodass die Hoffnung besteht, dass der Einfluss von ROCK-Inhibition in Zukunft möglicherweise auch in vivo bei der HD nachgewiesen wird (Hensel et al. 2015).

Auch im Bereich der Spinalen Muskelatrophie (SMA) spielt eine erhöhte ROCK-Aktivität eine Rolle. SMA ist eine monogenetische Erkrankung und wird durch eine loss-of-functionMutation im survival of motoneuron (Smn) 1-Gen ausgelöst. Weil Smn-1 ubiquitär exprimiert wird, wird SMA mittlerweile als Multisystemerkrankung gesehen, bei der jedoch die Motoneurone als erstes betroffen sind (Shababi et al. 2014). In Zellkulturen konnte nachgewiesen werden, dass der Verlust von Smn-1 direkt zu einer Überaktivierung der Rho-ROCK-Signalkaskade und vermindertem axonalen Wachstum führt und eine ROCKInhibition diesem Mechanismus entgegenwirken kann (Bowerman et al. 2007). In einem SMA-Mausmodell konnte eine erhöhte ROCK-Aktivität im Rückenmark nachgewiesen werden und durch eine orale Behandlung mit den ROCK-Inhibitoren Y-27632 und Fasudil das Überleben der Tiere signifikant verlängert werden, die Morphologie der motorischen Endplatten erhalten und die muskuläre Atrophie aufgehalten werden (Bowerman et al. 2010, Bowerman et al. 2012). Neben dem ROCK-Signalweg ist im Rückenmark von Tieren 
des SMA-Mausmodells auch die ERK-Signalachse überaktiviert, was sich hier im Phänotyp der SMA - entgegen der Annahme ERK habe eine neurotrophe, antiapoptotische Wirkung - schädlich auswirkt, weil eine ERK-Inhibition durch Selumetinib das Überleben der Mäuse verlängert (Branchu et al. 2013). Eine gegenseitige Beeinflussung des ROCK- und des ERK-Signalweges wurde bei der SMA in vitro nachgewiesen, sodass die Autoren eine kombinierte Behandlung mit ROCK- und ERK-Inhibitoren vorschlagen (Hensel et al. 2014).

\subsubsection{ROCK-Inhibition bei ALS}

Die zellulären und molekularen Mechanismen, die für die neuroprotektiven Effekte der ROCK-Inhibition verantwortlich sind, spielen auch in der ALS eine entscheidende Rolle.

In unserer Forschungsgruppe wurde die klinische Testung und die histologische Evaluation des SOD1G93A-Mausmodell für die ALS etabliert (Günther et al. 2012), um Effekte der oralen Behandlung mit ROCK-Inhibitoren in vivo zu testen (Günther et al. 2014, Tönges et al. 2014, Günther 2015, Suhr 2017).

In der Pathogenese der ALS spielt eine erhöhte ROCK-Aktivität eine entscheidende Rolle: In der Zellkultur der Mouse Motor Neuron-Like Hybrid (NSC-34)-Zelllinie konnte nach SOD1G93A-Transfizierung eine signifikant erhöhte ROCK-Aktivität gezeigt werden und auch im SOD1G93A-Mausmodell konnte eine erhöhte ROCK-Aktivität indirekt durch ein erhöhtes phoyphoryliertes Akt (pAkt) in Lysaten des Rückenmarks nachgewiesen werden. Daneben konnten eine erhöhte PTEN-Aktivität und eine dem entsprechende Herabregulation des neurotrophen Akt-Signalweges in der SOD1G93A-transfizierten NSC-34-Zelllinie nachgewiesen werden, welche durch ROCK-Inhibition mit Fasudil wieder aufgehoben werden konnte. Dies geht im mit Fasudil behandelten SOD1G93AMausmodell mit einem verspäteten Symptombeginn, verlängertem Überleben und einer teilweisen erhaltenen Motoneuronanzahl im Rückenmark einher (Takata et al. 2013).

Wir konnten darüber hinaus im SOD1G93A-Mausmodell unter Fasudil-Therapie auch eine verbesserte motorische Performance feststellen. Zudem wurde die MotoneuronDegeneration aufgehalten, und Nervenfasern waren weniger beschädigt. Während eine verstärkte Astrogliose unter der Therapie festgestellt wurde, waren mehr Mikrogliazellen im vorteilhaften M2-Zustand (Tönges et al. 2014). Diese Beobachtung konnte zuvor bereits in vitro demonstriert werden (Zhang et al. 2013). In Zellkultur von lumbalen B6/SJLMotoneuronen, die im Rahmen eines mit Kainat induzierten Modells für Glutamatexzitotoxizität kultiviert wurden, zeigte sich darüber hinaus ein signifikant besseres Überleben der Motoneurone unter Fasudil-Therapie (Tönges et al 2014). 
Takata et al. (2013) konnten für den ROCK-Inhibitor Fasudil im SOD1G93A-Mausmodell sowohl in der Dosierung von $30 \mathrm{mg} / \mathrm{kg}$ Körpergewicht (KG) als auch in der Dosierung von $100 \mathrm{mg} / \mathrm{kg}$ KG einen verspäteten Krankheitsbeginn und ein verlängertes Überleben nachweisen. Ein verbessertes Überleben der Motoneurone im Alter von 15 Wochen untersuchten die Autoren jedoch nur bei einer Dosierung von $30 \mathrm{mg} / \mathrm{kg} \mathrm{KG}$, ebenso wie den Einfluss auf pAkt. Dies geht einher mit unseren Beobachtungen, dass nur die Dosierung von $30 \mathrm{mg} / \mathrm{kg}$ KG einen signifikanten Einfluss auf das Überleben und die motorische Performance im SOD1G93A-Mausmodell hat und sich sogar in unseren Ergebnissen signifikant von denen mit 100 mg/kg KG behandelten Mäusen unterscheidet (Tönges et al. 2014).

Daher wirkt Fasudil im ALS-Modell dosisabhängig und erreicht seine Wirkung durch zwei Mechanismen: Einerseits durch die Beeinflussung der Mikroglia und andererseits durch die Beeinflussung des neurotrophen PTEN/Akt-Signalweges. Die Annahme, neuroprotektive Effekte bei der ALS durch die Beeinflussung des Rho-ROCK-Signalweges erreichen zu können, wurde auch durch die Erkenntnis unterstützt, dass Mutationen im Profilin-1-Gen mit einem hohen Risiko, an ALS zu erkranken, assoziiert sind (Wu et al. 2012). Die mit ALS assoziierte Mutation Profilin1 ${ }^{\text {T109M }}$ betrifft die $\mathrm{zu}$ phosphorylierende Domäne des Proteins, was die These unterstützt, eine mangelnde Aktivierung von Profilin-1 wirke sich verschlechternd auf die ALS aus (Ingre et al. 2013). Dennoch ist nicht gesichert, ob der Rho-ROCK-Signalweg auf genau diese Domäne einen aktivierenden Einfluss hat (Hensel et al. 2015).

\subsection{Zielsetzung der Arbeit}

Diese Studie wird als Fortführung der präklinischen Studien im SOD1G93A-Modell geplant. Da in Vorarbeiten die neuroprotektive Wirkung von Fasudil am SOD1G93AModell prinzipiell belegt werden konnte (Tönges et al. 2014), sollen in der vorliegenden Arbeit dosisabhängige Effekte genauer untersucht und hier auch eine niedrig dosierte Fasudil-Therapie zum Einsatz kommen. Darüber hinaus erfolgt eine kombinatorische Behandlung mit dem klinisch zugelassenen Riluzol, um mögliche Interaktionseffekte zu evaluieren.

Der Experimentalaufbau der vorliegenden Studie umfasst die folgenden Versuchsgruppen: niedrig dosierte Behandlung (10 mg/kg KG) mit Fasudil, mitteldosierte Behandlung (30 $\mathrm{mg} / \mathrm{kg} \mathrm{KG}$ ) mit Fasudil, niedrigdosierte Behandlung mit Fasudil in Kombination mit Riluzol und eine Monotherapie mit Riluzol sowie eine gesunde und eine unbehandelte Kontrollgruppe. Die therapeutischen Zielparameter umfassen die Evaluation der 
motorischen Performance incl. erstmals in unserem Labor bei Mäusen durchgeführter Ganganalyse mittels CatWalk XT gait analysis system sowie eine erstmalig in unserem Labor durchgeführte Evaluation der kognitiven Performance mittels novel object recognition test.

Die Auswertung der vorliegenden Studie erfolgt im Rahmen einer Verlaufsbeurteilung der klinischen Entwicklung sowie mittels Querschnittstudie mit histologischer Evaluation der Pathologie spinaler Motoneurone und Atrophie des Musculus gastrocnemius.

Die Ziele der vorliegenden Arbeit sind somit in Zusammenfassung:

1. den Effekt einer niedrig dosierten Fasudil-Therapie (10 $\mathrm{mg} / \mathrm{kg} \mathrm{KG}$ ) mit der mitteldosierten Therapie (30 mg/kg KG) zu vergleichen, die Kombination mit Riluzol dieser Therapie zu evaluieren und die verschiedenen Dosierungen bzw. die Kombination mit einer Riluzol-Monotherapie zu vergleichen,

2. die Auswirkung der gewählten Therapie auf das Überleben, die klinische Entwicklung, die anhand des Gewichts und eines klinischen Scores evaluiert wird, die motorische Performance, die mithilfe des Drehwalzenversuchs (Rotarod) und mithilfe eines videobasierten Ganganalysesystems (Catwalk XT) analysiert wird und die kognitive Entwicklung, die mit dem novel object recognition test (NOR) evaluiert wird, in einer Verlaufsstudie zu untersuchen,

3. die Auswirkung der gewählten Therapie auf die Anzahl der Alpha-Motoneurone im lumbalen Rückenmark und die Atrophie des Musculus gastrocnemius in einer Querschnittstudie zu untersuchen. 


\section{Material und Methoden}

\section{$2.1 \quad$ Geräte}

\begin{tabular}{|c|c|}
\hline Geräte & Hersteller/Bezug \\
\hline Axioplan Fluoreszenzmikroskop & $\begin{array}{l}\text { Axioplan 2, Carl Zeiss Microscopy GmbH, Jena, } \\
\text { Deutschlandišpi }\end{array}$ \\
\hline Axioplankamera & $\begin{array}{l}\text { AxioCam HRm, Carl Zeiss Microscopy GmbH, Jena, } \\
\text { Deutschland }\end{array}$ \\
\hline Catwalk XT & $\begin{array}{l}\text { Catwalk XT gait analysis system, Noldus, Wageningen, } \\
\text { Niederlande }\end{array}$ \\
\hline Eismaschine & AF 100, Scotsman Ice Systems, Milano, Italien \\
\hline Feinwaage bis $0,01 \mathrm{~g}$ & LE6202S, Sartorius AG, Göttingen, Deutschland \\
\hline Feinwaage bis $0,1 \mathrm{mg}$ & BL210S, Sartorius AG, Göttingen, Deutschland \\
\hline $\begin{array}{l}\text { Gefrierschnittmaschine } \\
\text { (Kryostat) }\end{array}$ & $\begin{array}{l}\text { CM-3050-S, Leica Biosystems GmbH, Nussloch, } \\
\text { Deutschland }\end{array}$ \\
\hline Gefrierschrank $\left(-20^{\circ} \mathrm{C}\right)$ & $\begin{array}{l}\text { Robert Bosch Hausgeräte GmbH, München, } \\
\text { Deutschland }\end{array}$ \\
\hline Gefrierschrank $\left(-80^{\circ} \mathrm{C}\right)$ & $\begin{array}{l}\text { Forma, ThermoQuest Analytische Systeme GmbH, } \\
\text { Egelsbach, Deutschland }\end{array}$ \\
\hline Gelelektrophoresekammer & BioRad Laboratories, München, Deutschland \\
\hline Inkubationsschrank $\left(37^{\circ} \mathrm{C}\right)$ & $\begin{array}{l}\text { Heraeus B20, Kendro Laboratory Products GmbH, } \\
\text { Langenselbold, Deutschland }\end{array}$ \\
\hline Kühlschrank $\left(5^{\circ} \mathrm{C}\right)$ & $\begin{array}{l}\text { Liebherr-Hausgeräte Ochsenhausen GmbH, Ochsen- } \\
\text { hausen, Deutschland }\end{array}$ \\
\hline Magnetrührer & $\begin{array}{l}\text { Ikamag RH, IKA-Werke GmbH \& CO. KG, Staufen, } \\
\text { Deutschland }\end{array}$ \\
\hline Mikrowelle & $\begin{array}{l}\text { Robert Bosch Hausgeräte GmbH, München, } \\
\text { Deutschland }\end{array}$ \\
\hline $\mathrm{pH}-$ Meter & $\begin{array}{l}\text { pH-Meter PD20, Sartorius AG, Göttingen, } \\
\text { Deutschland }\end{array}$ \\
\hline Pipetten $0,2-2 \mu 1,1-10 \mu 1,2-20$ & Pipetman Classic P2, P10, P20, P100, P200, P1000; \\
\hline
\end{tabular}




\begin{tabular}{|l|l|}
\hline $\begin{array}{l}\mu 1,20-100 \mu 1,50-200 \mu 1,200- \\
1000 \mu 1\end{array}$ & Gilson Inc., Middleton, Wisconsin, USA \\
\hline Reinstwasseranlage & $\begin{array}{l}\text { Elga Purelab Plus UV/UF PL5124, ELGA LabWater, } \\
\text { Celle, Deutschland }\end{array}$ \\
\hline Schwenktisch & $\begin{array}{l}\text { Stuart 3D gyratory rockers - SSM3, Bibby Scientific } \\
\text { Limited, Stone, Vereinigtes Königreich }\end{array}$ \\
\hline Videokamera: Legria HFM 36 & Canon, Ōta (Tokio), Japan \\
\hline Vortex Reagenzglasmischer & $\begin{array}{l}\text { 7-2020, neoLab Migge Laborbedarf-Vertriebs GmbH, } \\
\text { Heidelberg, Deutschland }\end{array}$ \\
\hline Wasserbad & $\begin{array}{l}\text { 3043, Köttermann GmbH \& Co KG, Hänigsen, } \\
\text { Deutschland }\end{array}$ \\
\hline
\end{tabular}

Tabelle 1. Geräte

\subsection{Verbrauchsmaterialien und allgemeine Chemikalien}

\begin{tabular}{|l|l|}
\hline $\begin{array}{l}\text { Verbrauchsmaterialien/ } \\
\text { Chemikalien }\end{array}$ & Hersteller/Bezug \\
\hline Agarose & AppliChem GmbH, Darmstadt \\
\hline Deckgläschen & $\begin{array}{l}\text { Gerhard Menzel Glasbearbeitungswerk GmbH \& Co } \\
\text { KG, Braunschweig Deutschland }\end{array}$ \\
\hline Gefriermatrix & $\begin{array}{l}\text { Shandon Cyromatrix, Thermo Fisher Scientific Inc, } \\
\text { Kalamazoo, Michigan, USA }\end{array}$ \\
\hline Objektträger & $\begin{array}{l}\text { ThermoScientific Superfrost Plus, Gerhard Menzel } \\
\text { Glasbearbeitungswerk GmbH \& Co KG, Braunschweig } \\
\text { Deutschland }\end{array}$ \\
\hline Paraformaldehyd & A3813, AppliChem GmbH, Darmstadt, Deutschland \\
\hline Phosphat buffered saline & A0964, AppliChem GmbH, Darmstadt, Deutschland \\
\hline Pipettenspitzen ohne Filter & Sarstedt AG \& Co., Nümbrecht, Deutschland \\
\hline Reagenzröhre (25 ml, 50 ml) & Sarstedt AG \& Co., Nümbrecht, Deutschland \\
\hline $\begin{array}{l}\text { Safe-Lock-Reaktionsgefäße (0,5 } \\
\text { ml, 1,5 ml, 2 ml) }\end{array}$ & Sarstedt AG \& Co., Nümbrecht, Deutschland \\
\hline Mowiol & Hoechst, Frankfurt, Germany \\
\hline
\end{tabular}

Tabelle 2. Verbrauchsmaterialien und allgemeine Chemikalien 


\subsection{Studiendesign}

Tierexperimentelle Studien sind im Rahmen präklinischer Studien der ALS von großer Bedeutung und das SOD1G93A-Mausmodell stellt hier einen Standard für präklinische Studien der ALS dar. Das verwendete Mausmodell ist in der ALS-Forschung seit seiner Entwicklung ein etabliertes und vielfach untersuchtes Modell und sticht durch seine weitgehende klinische und neuropathologische Vergleichbarkeit mit der familiären und auch der sporadischen ALS beim Menschen hervor (Ludolph et al. 2010). Die Methoden und Ergebnisse dieser Studie werden dem CONSORT Statement für randomisierte Versuche paralleler Gruppen folgend geplant und werden dementsprechend präsentiert (Moher et al. 2010). Die Experimente mit Tieren werden nach den Maßgaben des Tierschutzgesetzes (Bundesgesetzblatt (BGBl.) I S.1206ff Jahrgang 2006) durchgeführt (Genehmigungsbescheid der Tierversuche, Aktenzeichen: 33.9-42502-04-12/0938). Der Untersucher ist den Federation of European Laboratory Animal Science Associations (FELASA)-BRichtlinien entsprechend zertifiziert, Tierversuche durchführen zu dürfen. Dem Untersucher wurde durch das Niedersächsische Landesamt für Verbraucherschutz und Lebensmittelsicherheit genehmigt, die im Rahmen dieser Arbeit geplanten verhaltensbiologischen Eingriffe unter Anleitung an Tieren durchzuführen (Nieders. Landesamt für Verbraucherschutz und Lebensmittelsicherheit, Aktenzeichen: 33.4-4350214-Scheer). Es wird einerseits eine Verlaufsstudie zur Kontrolle der klinischen und verhaltensbiologischen Parameter unter präsymptomatischer Behandlung durchgeführt. Andererseits wird eine Querschnittsstudie zur makroskopischen Evaluation der Muskelatrophie und einer histologischen Evaluation der spinalen Motoneurone nach präsymptomatischer Behandlung durchgeführt. Aufgebaut wird die Studie auf den Erkenntnissen und Erfahrungen, die im Rahmen unserer Forschungsgruppe und der darin enthaltenen ALS-Arbeitsgruppe bereits gewonnen wurden, insbesondere im Umgang mit dem SOD1G93A-Mausmodell (Günther et al. 2012, Tönges et al. 2014, Günther et al. 2014, Günther 2015, Suhr 2017, Günther et al. 2017).

\subsection{Tierzucht und Tierhaltung}

Das von Gurney et al. (1994) entwickelte High-copy B6SJL-Tg(SOD1-G93A)1Gur/J Genotyp Mausmodell (Jackson Labs, Bar Harbor, USA) wird als Grundlage für die Versuchsanordnung verwendet. Vier von Jackson Lab georderte transgene Böcke werden in der Zentralen Tierexperimentellen Einrichtung der Universitätsmedizin Göttingen (ZTE) mit B6SJLF1/J-Wildtyp-Weibchen (Jackson Lab, Bar Harbor, USA) verpaart. Da durch den schnellen Krankheitsprogress die Fortpflanzungsfähigkeit der Böcke zeitlich 
sehr begrenzt ist, ist es nötig, wie in den vorhergehenden Studien an diesem Modell, zur weiteren Erhaltung der Zucht die transgenen Böcke der Folgegeneration mit den B6SJLF1/J-Wildtyp-Weibchen zu verpaaren. Hierbei wird eine direkte Verwandtschaft zwischen Böcken und Verpaarungsweibchen ausgeschlossen. Durch die Verpaarung von den heterozygoten transgenen Böcken mit Wildtypen ist lediglich etwa die Hälfte der Nachkommen ebenfalls transgen. Zur Untersuchung werden nur weibliche Tiere genutzt, weil sie einen etwas längeren, weniger rapiden Krankheitsverlauf als die Männchen haben (Heiman-Patterson et al. 2005). Dadurch ergibt sich eine längere Möglichkeit der oralen Behandlung. Es wird bei den Weibchen durch den langsameren Krankheitsverlauf eine erhöhte Sensibilität für therapeutische Interventionen vermutet. Zudem werden einige transgene männliche Tiere für die Zuchterhaltung genutzt.

Zur Genotypisierung werden den Mäusen zwei bis drei Wochen nach Geburt Schwanzspitzen mit einer Länge von circa 0,4 cm entnommen und per Botendienst aus der ZTE an die experimentelle Einrichtung (Neurobiologisches Labor Prof. Bähr, Waldweg 33, Göttingen, Deutschland) gesendet. Es wird dort die Genotypisierung mittels Polymerasekettenreaktion und nachfolgender Gelelektrophorese durchgeführt.

Nach der erfolgten Genotypisierung und geplanter Einteilung in die Versuchsgruppen werden die benötigten Tiere von der ZTE im Alter von ca. 33 bis 43 Tagen in die experimentelle Einrichtung transportiert. Sowohl in der ZTE als auch in der experimentellen Einrichtung erfolgt die Haltung und Pflege der Tiere in individually vetilated cages (IVC) durch ausgebildete Tierpfleger der ZTE. In den IVC ist mittels der separaten Käfigbelüftung eine dauernde Luftfeuchtigkeit von 60\% und eine Raumtemperatur von $23{ }^{\circ} \mathrm{C}$ garantiert, die ebenfalls im Versuchsraum herrschen. Die Versorgung mit Flüssigkeit ist an den Käfigen durch angebrachte Trinkbehälter verwirklicht, die vom Untersucher regelmäßig selber erneuert werden, um die therapeutische Behandlung der Versuchsgruppen über das Trinkwasser zu kontrollieren. Das Futter der Tiere besteht aus einer vollwertigen Alleinfuttermischung (ssniff Spezialdiäten GmbH, Soest, Deutschland). Diese ist für die Tiere als Trockenpellet durch die Gitterabdeckung des Käfigs erreichbar. In der späten Krankheitsphase, bei Erreichen des Punktwert 2 des klinisch-neurologischen Scores (siehe Kapitel 2.7.1.1) werden die Pellets mit der Flüssigkeit aus dem entsprechenden Trinkbehältnis der Behandlungsgruppe zu Brei aufgeweicht und in einer Plastikschale am Käfigboden angeboten. Dadurch wird eine Nahrungsaufnahme sowie die Flüssigkeits- und Medikamentenzufuhr auch in der letzten Krankheitsphase gewährleistet. Die Tiere werden in einem 12-stündigen Hell-/Dunkelrhythmus gehalten. Die verhaltensbiologischen Experimente werden möglichst in oder nahe an der Aktivitätsphase 
der nachtaktiven Tiere in der Dunkelheit zu immer derselben Uhrzeit durchgeführt. Damit die Tiere bereits am ersten Testtag, dem 50. Lebenstag, an den Untersucher und die Untersuchungsgeräte gewöhnt sind und um Angst zu vermeiden, wird mit allen Tieren mindestens zweimal innerhalb einer Woche vor dem Versuchsbeginn der volle Versuchsablauf des Drehwalzenversuchs durchgeführt (siehe Kapitel 2.7.2).

\subsection{Genotypisierung}

Um die DNA aus dem Gewebe der Schwanzspitzen freizusetzen, werden sie mit je $160 \mu \mathrm{l}$ DirectPCR-Lyse-Reagenz (Peqlab Biotechnologie GmbH, Erlangen, Deutschland) und je 3 $\mu$ Proteinkinse K (10 mM in Tris, pH 8, AppliChem, Darmstadt, Deutschland) über 12 Stunden bei $55{ }^{\circ} \mathrm{C}$ inkubiert. Um diese Reaktion $\mathrm{zu}$ stoppen werden die Proben anschließend für 45 Minuten bei $85^{\circ} \mathrm{C}$ denaturiert. Nach der Abkühlung, werden je $2 \mu \mathrm{l}$ des vorliegenden Lysates, einer internen negativen Kontrolle sterilen Wassers und das Lysat eines bereits genotypisierten Tieres als Kontrolle in zwei 0,5-ml-EppendorfReaktionsgefäße überführt. Zu jedem Lysat werden $18 \mu$ l eines Mastermix hinzugegeben. Dieser setzt sich aus $2 \mu \mathrm{l}$ dNTP (Desoxynukleosid-Triphosphat-Mixlösung, $2 \mathrm{mM} / \mathrm{dNTP}$; Biolab GmbH, München, Deutschland), $2 \mu$ l eines zehnfachen PCR-Reaktionspuffers (Peqlab Biotechnology GmbH, Erlangen, Deutschland), zur Amplifizierung der transgenen SOD $1 \quad 0,25 \quad \mu l$ eines Vorwärts-Primers mit $\operatorname{der}$ Sequenz 5 'CATCAGCCCTAATCCATCTGA- 3’ (100 $\mu \mathrm{M}$, Sigma-Aldrich, München, Deutschland) und $0,25 \quad \mu l$ eines Rückwärts-Primer mit der Sequenz 5 'CGCGATTAACAATCAAAGTGA-3 ' (100 $\mu \mathrm{M}$ ， Sigma-Aldrich, München), zur Amplifizierung von Interleukin 2 zur internen positiven Kontrolle 0,25 $\mu \mathrm{l}$ eines VorwärtsPrimers mit der Sequenz 5'-CTAGGCCACAGAATTGAAAGATCT- 3' (100 $\mu \mathrm{M}$, SigmaAldrich, München, Deutschland), 0,25 $\mu$ l eines Rückwärts-Primer mit der Sequenz 5'GTAGgTGgAAATTCTAGCATCAT- 3', 0,1 $\mu$ l Taq-Polymerase (Rekombinante DNAPolymerase, interne Laborherstellung) in 12,9 $\mu$ l sterilem Wasser zusammen.

Das vorliegende Gemisch wird in einem PCR-Gerät (Cyclone 25, Peqlab Biotechnology GmbH, Erlangen, Deutschland) nach folgendem Schema amplifiziert: Das Gerät wird auf $110{ }^{\circ} \mathrm{C}$ vorgeheizt; Initialisierung der DNA-Denaturierung für 5 Minuten bei $94{ }^{\circ} \mathrm{C}$; Schleife für 30 Durchläufe mit jeweils $30 \mathrm{~min}$ bei $94{ }^{\circ} \mathrm{C}$, dann $30 \mathrm{~min}$ bei $55^{\circ} \mathrm{C}$ für Anlagerung der Primer an die entsprechende Region der Einzelstrang-DNA, dann 30 min bei $72{ }^{\circ} \mathrm{C}$ für die Transkription des spezifischen DNA-Abschnittes, erneuter Beginn der 
Reaktionsschleife; Beendigung der Amplifikation bei $72{ }^{\circ} \mathrm{C}$ für $5 \mathrm{~min}$, abschließend Abkühlung auf $4{ }^{\circ} \mathrm{C}$.

Zu dem Lysat werden nun $3 \mu$ Ladepuffer (15\% Ficoll 400 DL, 100 mM LiCl, 100 mM EDTA (Ethylendiamintetraessigsäure), 0,05 \% Bromphenol, in $50 \mathrm{ml} \mathrm{H} 2 \mathrm{O}$ ) gegeben und ca. $15 \mu \mathrm{l}$ jeder Probe auf ein 1,5\% Agarosegel aufgetragen. Die beiden Proben eines Tieres werden jeweils übereinander oder nebeneinander aufgetragen und bei der Auswertung verglichen, um die Validität der Genotypisierung zu erhöhen und falsche Ergebnisse oder Verunreinigungen zu erkennen.

Die Herstellung des Gels erfolgt mit 1,2 g Agarose (AppliChem GmbH, Darmstadt) pro 60 ml einfacher TBE-Lösung (Tris- Base-EDTA-Puffer, AppliChem GmbH, Darmstadt, Deutschland). Die Agarose wird in einer Mikrowelle durch kurzes Sieden aufgelöst und anschließend mit 1,2 $\mu \mathrm{l}$ Ethidiumbromid $(10 \mathrm{mg} / \mathrm{ml}$, Sigma-Aldrich Chemie $\mathrm{GmbH}$, München, Deutschland) versetzt und durch kurzes Schwenken verteilt. Die Flüssigkeit wird in einen Gelschlitten (BioRad Laboratories, München, Deutschland) gegeben und zwei passende Kämme (BioRad Laboratories, München, Deutschland) zur Taschenformung darin eingetaucht. Nach Abwarten von ca. einer Stunde ist das Gel erkaltet und in einem festen Aggregatzustand. Es wird daraufhin in einer Gelelektrophoresekammer (BioRad Laboratories, München, Deutschland) in TBE-Pufferlösung versenkt. In die Taschen wird, nachdem auch sie mit TBE-Pufferlösung gefüllt sind, das Probenreagenz in o. g. Anordnung gegeben.

Für ca. 45 Minuten wird an die Gelelektrophoresekammer eine Spannung von 80 Volt angelegt. Dabei wird die negativ geladene DNA zur Anode gezogen und durchwandert das Gitternetzwerk der Gelmatrix des Agarosegels. Dabei laufen die DNA-Fragmente abhängig von ihrer Größe schneller oder langsamer. In die jeweils erste Kammer wird zudem eine DNA-Leiter (100 ng/ $\mu$ l, Dianova, Hamburg, Deutschland) gegeben, anhand derer man die Länge der sich im Gel aufteilenden DNA-Fragmente bestimmen kann. Mithilfe des an die DNA gebundenen Ethidiumbromids, das unter UV-Licht sichtbar wird, kann durch einen an eine Kamera angeschlossenen Computer mit dem Computerprogramm Quantity-One 4.2.2 (BioRad Laboratories, München, Deutschland) die DNA sichtbar gemacht werden.

Das amplifizierte Genprodukt der SOD1 mit der G93A-Punktmutation wird durch eine Bande im Bereich von 236 Basenpaaren (bp) sichtbar. Um ein falsch negatives Ergebnis durch fehlende DNA in Probe zu erkennen, ist das Genprodukt von Interleukin-2 bei 324 bp sichtbar (siehe Abbildung 3). 


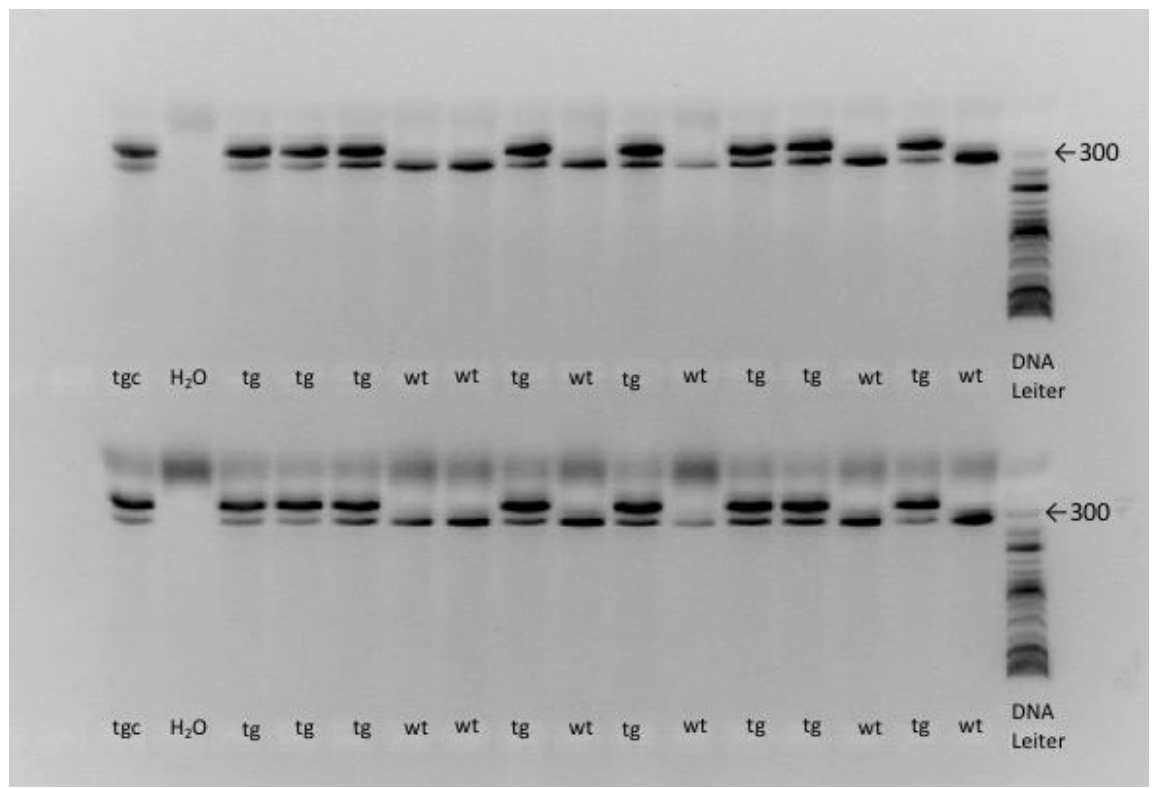

Abbildung 3. Genotypisierung der SOD1G93A-transgenen Tiere

Abgebildet sind die durch Ethidiumbromid im UV-Licht sichtbaren amplifizierten Genprodukte im Agarosegel nach durchgeführter Gelelektrophorese: DNA Leiter mit Markierung (Pfeil) für 300 bp, interne negative Kontrollprobe Wasser $\left(\mathrm{H}_{2} \mathrm{O}\right)$, transgene Kontrolle (tgc), Tiere mit SOD1-Wildtyp (wt) mit Bande des amplifizierten Interleukin 2 als interne positive Kontrolle bei 324 bp, SOD1G93A-transgene Tiere (tg) mit Banden des amplifizierten Interleukin und des amplifizierten Genprodukts mit SOD1G93A-Punktmutation bei 236 bp (Quelle: eigenes Bildmaterial).

\subsection{Versuchsgruppen}

Nachdem die Tiere eines Wurfes genotypisiert wurden, werden die transgenen weiblichen Tiere in fünf Gruppen randomisiert: Vehikel ohne Behandlung (Veh), Riluzol 30mg/kg KG/d (Ril30), Fasudil $10 \mathrm{mg} / \mathrm{kg} \mathrm{KG} / \mathrm{d}$ (Fas10), Fasudil $30 \mathrm{mg} / \mathrm{kg} \mathrm{KG} / \mathrm{d}$ (Fas30), Kombinationsbehandlung (Fas10Ril30) (siehe Tabelle 3). Wurfgeschwister werden auf die verschiedenen Gruppen aufgeteilt. Die männlichen transgenen Tiere werden für die Zuchterhaltung benötigt. Nicht-transgene weibliche Mäuse vom SOD1-Wildtyp werden für die Wildtypkontrollgruppe (Wt) verwendet. Alle übrigen Tiere werden nach der erfolgten Genotypisierung und Aufteilung euthanasiert. Die Aufteilung erfolgt für den Untersucher verblindet, indem die entsprechenden Trinkbehältnisse vom Untersucher mit der vorgesehenen Behandlung bestückt und beschriftet werden. Bis nach der statistischen Auswertung durch den Untersucher wird diese Verblindung und die Zuordnung beibehalten.

\subsubsection{Verlaufsstudie: Verhaltensbiologie}

Die für die Studie ausgewählten Tiere werden zusammen mit gleichaltrigen Tieren „gematcht" und in ihren entsprechenden Gruppen in IVC-Käfigen mindestens eine Woche vor Versuchsbeginn aneinander gewöhnt und mithilfe der zweimaligen Durchführung des Drehwalzenversuchs auch an den Untersucher und die verhaltensbiologischen Testungen 
gewöhnt. Es werden pro Gruppe zwölf Tiere untersucht. In der Wt-Gruppe werden sieben Tiere als Kontrolle untersucht. Es wird eine klinische Analyse des Überlebens, des Krankheitsverlaufs und des Körpergewichts durchgeführt. Es wird eine Analyse der motorischen Performance mithilfe des Drehwalzentests (Rotarod) und mithilfe einer videobasierten Ganganalyse (Catwalk XT) durchgeführt. Zudem wird eine Analyse der kognitiven Leistung (novel object recognition (NOR)-Test) durchgeführt.

\begin{tabular}{|c|c|c|c|c|c|c|}
\hline \multirow[b]{2}{*}{ Bezeichnung } & \multicolumn{6}{|c|}{ Versuchsgruppen } \\
\hline & Veh & Fas10 & Fas30 & Ril30 & $\begin{array}{l}\text { Fas10 } \\
\text { Ril30 }\end{array}$ & $\mathbf{W t}$ \\
\hline $\begin{array}{c}\text { Behandlung } \\
\text { in } \mathrm{mg} / \mathrm{kg} \\
\mathrm{KG} / \mathrm{Tag}\end{array}$ & $\begin{array}{c}\mathrm{H}_{2} \mathrm{O} \\
\text { Wasser }\end{array}$ & Fasudil 10 & Fasudil 30 & Riluzol 30 & $\begin{array}{l}\text { Fasudil } 10 \\
\text { Riluzol } 30\end{array}$ & $\begin{array}{c}\mathrm{H}_{2} \mathrm{O} \\
\text { Wasser }\end{array}$ \\
\hline Genotyp & $\mathrm{SOD} 1^{\mathrm{G} 93 \mathrm{~A}}$ & $\mathrm{SOD} 1^{\mathrm{G} 93 \mathrm{~A}}$ & $\mathrm{SOD} 1^{\mathrm{G} 93 \mathrm{~A}}$ & $\mathrm{SOD} 1^{\mathrm{G} 93 \mathrm{~A}}$ & $\mathrm{SOD} 1^{\mathrm{G} 93 \mathrm{~A}}$ & $\mathrm{SOD} 1^{\mathrm{Wt}}$ \\
\hline
\end{tabular}

Tabelle 3. Versuchsgruppenübersicht

\begin{tabular}{|c|c|c|c|c|c|c|c|}
\hline \multirow[b]{2}{*}{ Experiment } & \multicolumn{6}{|c|}{ Versuchsgruppen } & \multirow{2}{*}{$\begin{array}{l}\text { Behandlungs- } \\
\text { zeitraum } \\
\text { Beginn/Ende }\end{array}$} \\
\hline & Veh & Fas10 & Fas30 & Ril30 & $\begin{array}{l}\text { Fas10 } \\
\text { Ri130 }\end{array}$ & $\mathbf{W t}$ & \\
\hline \multirow{2}{*}{$\begin{array}{l}\text { Querschnittstudie: } \\
\text { Histologie } \\
\text { Muskelatrophie }\end{array}$} & $\mathrm{n}=5$ & $\mathrm{n}=5$ & $\mathrm{n}=5$ & $\mathrm{n}=5$ & $\mathrm{n}=5$ & $\mathrm{n}=5$ & \multirow[t]{2}{*}{ Tag 50/ Tag 120} \\
\hline & $\mathrm{n}=5$ & $\mathrm{n}=5$ & $\mathrm{n}=5$ & $\mathrm{n}=5$ & $\mathrm{n}=5$ & $\mathrm{n}=10$ & \\
\hline $\begin{array}{l}\text { Verlaufsstudie: } \\
\text { Verhaltensbiologie }\end{array}$ & $\mathrm{n}=12$ & $\mathrm{n}=11 *$ & $\mathrm{n}=12$ & $\mathrm{n}=12$ & $\mathrm{n}=12$ & $\mathrm{n}=7$ & $\begin{array}{c}\text { Tag } 50 / \\
\text { Abbruchkriterien } \\
\text { erfüllt }\end{array}$ \\
\hline
\end{tabular}

*nachträglich wurde ein Tier zensiert, weil es zunächst falsch positiv genotypisiert wurde

\subsubsection{Querschnittstudie: Evaluation der spinalen Motoneurone und der Muskelatrophie}

Die für diese Studie ausgewählten Tiere werden ebenfalls zusammen mit gleichaltrigen Tieren in den entsprechenden Gruppen in IVC-Käfigen aufgeteilt gehalten und zusammen mindestens eine Woche vor dem Behandlungsbeginn aneinander gewöhnt. In jede Gruppe werden mindestens acht Tiere eingeschlossen, um für die histologische Querschnittsstudie zur Evaluation der Alpha-Motoneurone am Tag 120 in jeder Gruppe Gewebe von 
mindestens fünf Tieren auswerten zu können. Für die Evaluation der Atrophie des Musculus gastrocnemius werden in der Veh-Gruppe und den Behandlungsgruppen jeweils 5 Tiere untersucht. In der Wt-Gruppe werden als Kontrolle 10 Tiere untersucht.

\subsection{Verlaufsstudie: Verhaltensbiologie}

\subsubsection{Klinische Analyse: Überleben, Krankheitsverlauf und Körpergewicht}

2.7.1.1 Analyse des Krankheitsverlaufs, Versuchsabbruchkriterien und Überlebensanalyse Um den Krankheitsverlauf möglichst genau zu beobachten, zu dokumentieren und das Leid der Tiere zu minimieren, wird ab Behandlungsbeginn zweimal pro Woche ein klinisch-neurologischer Score nach Weydt et. al. (2003) erhoben. Nach Erreichen des Punktwerts 1 wird eine tägliche Erhebung durchgeführt. Dabei wird folgendes Punktesystem, das in unserer Forschungsgruppe in dieser Formulierung etabliert wurde, zugrunde gelegt (Günther et al. 2012, Günther 2015):

„4 - keinerlei neurologische Defizite, Wildtyp-ähnliche Verhaltensweise, vollständige Beinstreckung/Spreizung weg von der lateralen Mittellinie, während die Maus kopfüber am Schwanz gehalten wird

3 - Tremor der Hinterläufe und/oder Einschränkung/Abbruch der spontanen Beinstreckung weg von der lateralen Mittellinie, während die Maus kopfüber am Schwanz gehalten wird

2 - Gestörtes Gangbild im Sinne eines „Watschelgang“, Gang auf allen vier Extremitäten noch möglich; *

1 - Lähmung mindestens einer hinteren Extremität, wobei diese beim Laufen hinterhergezogen wird. Die Vorderläufe sind nicht betroffen. Das Tier ist in der Lage, selbstständig Futter zu erreichen und aufzunehmen. Das Tier dreht sich innerhalb 30 Sekunden selbstständig von der Rücken- in die Bauchlage.

0 - Unfähigkeit des Tieres, sich selbstständig in weniger als 30 Sekunden aus der Rücken- in die Bauchlage zu drehen oder es sind Lähmungserscheinungen in mehr als zwei Extremitäten sichtbar oder das Tier ist nicht in der Lage, selbstständig Futter oder Wasser zu sich zu nehmen oder das Tier hat mehr als $25 \%$ des Maximalgewichtes verloren oder das Tier hat über 48 Stunden einen Gewichtsverlust von über $20 \%$ des Maximalgewichts ohne dass in dieser Zeit eine erneute Gewichtszunahme erreicht werden konnte. 
* ab dem Punktwert 2 werden Futterpellets mit Wasser zu Brei aufgeweicht und in einer Plastikschale am Käfigboden platziert“ (Günther 2015, S. 35f)

Bei Erreichen des Punktwertes 0 auf dem vorliegenden Punkteschema wird durch eine sofortige Kohlendioxid-Insufflation die Euthanasie des Tieres durchgeführt. Der Lebenstag der Tötung wird als Überlebenszeit in die Auswertung eingebracht. Der Beginn der Krankheit ist mit dem erstmaligen Auftreten des Scores 3 dokumentiert und der Zeitraum zwischen diesem und dem Tötungstag wird als Krankheitsdauer gewertet. Die zeitliche Entwicklung des klinischen Scores in den Behandlungsgruppen geht in die Auswertung mit ein.

\subsubsection{Körpergewicht}

Durch die engmaschige Kontrolle des Körpergewichts der Mäuse kann allgemeiner Gesundheitszustand und Gedeihen gut kontrolliert und dokumentiert werden. Es kann die regelrechte Futteraufnahme und Flüssigkeitszufuhr, mögliche Störungen bei diesem, der Krankheitsprogress der transgenen Tiere und mögliche Unverträglichkeiten bei der Behandlung und andere Gesundheitsstörungen der Mäuse erfasst werden. Ebenfalls geht der Gewichtsverlauf in die Versuchsabbruchkriterien ein (Score 0, siehe Kapitel 2.7.1.1). Die Kontrolle des Gewichts erfolgt bei den Tieren mindestens zweimal die Woche, bei Erreichen des Scores 1 täglich. Die regelmäßigen Kontrollen von klinischem Score und Gewicht werden an einem festem dem Tag-/Nachtrhythmus der Tiere angepassten Tageszeitpunkt vorgenommen.

2.7.1.3 Statistische Auswertung des Überlebens, des Krankheitsverlaufs und des Gewichts Die erhobenen Parameter werden mit der Software Excel Version 2013 (Microsoft, Redmond, Vereinigte Staaten) analysiert und Grafiken erstellt. Zur statistischen Analyse der Parameter wird die Software SPSS Version 23 (IBM, Armonk, Vereinigte Staaten) genutzt.

Bei der Überlebensanalyse werden die Lebensendzeitpunkte mit einer Kaplan-Meier-Kurve dargestellt und mit einem log-Rank-test auf signifikante Unterschiede untersucht.

Zur Untersuchung des Krankheitsverlaufes wird zunächst der Zeitpunkt des Krankheitsbeginns ermittelt, also das erstmalige Auftreten des Score 3. Von diesen Zeitpunkten wird der Mittelwert gebildet und mit einer univarianten analysis of variance (ANOVA) und dem Least-Significance-Difference-Test (LSD-Test) auf signifikante Unterschiede geprüft. Ebenso wird mit den Zeitpunkten des erstmaligen Auftretens von Score 2, Score 1 und Score 0 (Endpunkt der Erkrankung/Tod) verfahren. Die im Ergebnisteil genannten Werte sind Mittelwerte +/- Standardfehler, ebenso wie die in Grafiken gezeigten Werte mit Fehlerbalken. 
Die Gewichtsdaten werden statistisch mithilfe einer ANOVA mit Messwiederholungen analysiert und signifikante Unterschiede durch den LSD-Test untersucht.

In die statistische Analyse der Gewichtsdaten gehen die erhobenen Daten von 22 Messtagen zwischen dem Lebenstag 50 und Lebenstag 123 mit ein, um auch am letzten analysierten Testtag von allen Behandlungsgruppen Messwerte von zehn oder mehr Individuen miteinfließen lassen zu können.

\subsubsection{Analyse der Motorkoordination mittels Drehwalzentest (Rotarod)}

\subsubsection{Motorische Testung: Durchführung des Drehwalzentests (Rotarod)}

Das Krankheitsbild der ALS ist maßgeblich durch eine motorische Beeinträchtigung charakterisiert. Dies gilt ebenso für das Erscheinungsbild der Tiere des SOD1G93AMausmodells. Um die motorische Beeinträchtigung der Mäuse möglichst genau zu objektivieren und zu dokumentieren wird der verhaltensbiologische Drehwalzentest in regelmäßigen Abständen zweimal pro Woche mit den Mäusen durchgeführt. Bereits eine Woche vor dem Behandlungsbeginn am 50. Lebenstag werden die Mäuse zweimal innerhalb einer Woche im Abstand von mindestens drei Tagen immer unter gleichen Bedingungen und zum gleichen Tageszeitpunkt mit dem kompletten Versuchsablauf vertraut gemacht. Dies dient dazu, bei Versuchsbeginn Angst zu vermeiden und die Tiere mit dem Untersucher und den verhaltensbiologischen Versuchen vertraut zu machen.

Der Drehwalzentest untersucht die Koordinationsfähigkeit und die motorische Balance der Tiere. Dabei können auf der Apparatur (Rota-Rod for mice 47600, UGO BASILE, Comerio Italien) bis zu fünf Individuen gleichzeitig getestet werden. Sie befinden sich dabei auf einer horizontal um die eigene Achse rotierenden Walze mit einem Durchmesser von drei Zentimetern. Auf der Walze sind Trennwände angebracht, die den direkten Kontakt zwischen den Tieren verhindern. Die Oberfläche der Walze aus Hartgummi besitzt längs verlaufende Riffel, die ein Festhalten der Mäuse beim Laufen auf der Walze ermöglichen. Fällt die Maus von der Walze, betätigt sie dadurch eine Taste unter der Walze und stoppt automatisch eine bei Versuchsbeginn gestartete Stoppuhr.

Bei Versuchsbeginn wird die Drehgeschwindigkeit bei 5 Drehungen pro Minute eingestellt und steigert sich gleichmäßig bis 40 Drehungen pro Minute am Ende der Versuchszeit, die 300 Sekunden beträgt. Es wird pro Laufkammer eine Maus platziert und der Versuch und die Stoppuhr sogleich gestartet. Zwischen den Läufen werden die Mäuse für mindestens 30 Minuten in ihren Heimatkäfig zurückgesetzt, um Stress und eine Erschöpfung zu vermeiden. Mit jedem Tier werden drei Durchläufe pro Testtag gemessen und der 
Mittelwert dieser drei geht in die Auswertung ein. Der maximal erreichbare Tageswert, der sich aus den drei Durchläufen ergibt, ist somit 300 Sekunden.

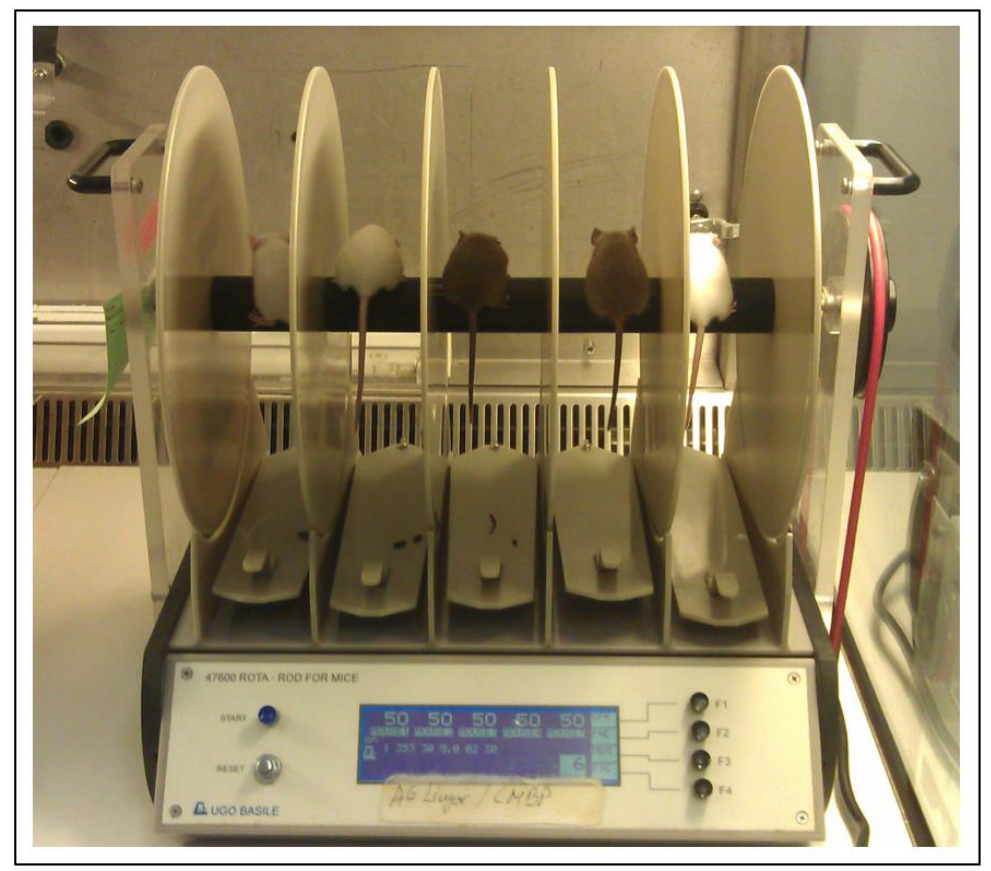

Abbildung 4. Versuchsaufbau des Drehwalzentests

Rota-Rod for mice 47600 (Quelle: eigenes Bildmaterial)

\subsubsection{Statistische Auswertung des Drehwalzentests}

Die im Drehwalzentest erhobenen Daten werden zunächst mit Excel erfasst und daraus Grafiken erstellt. Mit der SPSS Software werden die Daten auf signifikante Unterschiede untersucht. Die statistische Analyse erfolgt durch eine ANOVA mit Messwiederholungen und wird im Falle von Unterschieden mit dem LSD-Test auf Signifikanz untersucht. In die statistische Analyse gehen wie bei der Analyse des Körpergewichts die erhobenen Daten von 22 Messtagen zwischen dem Lebenstag 50 und Lebenstag 123 mit ein, um auch am letzten analysierten Testtag von allen Behandlungsgruppen Messwerte von zehn oder mehr Individuen miteinfließen lassen zu können. Die im Ergebnisteil genannten Werte sind Mittelwerte +/- Standardfehler, ebenso wie die in Grafiken gezeigten Werte mit Fehlerbalken.

\subsubsection{Videobasierte Analyse des Gangs bei sich frei bewegenden Mäusen (CatWalk XT)}

Im Rahmen der Erkrankung der transgenen Mäuse des SOD1G93A-Mausstammes ist der Gang starken Veränderungen unterworfen. Insbesondere sind die Hinterläufe einer frühen Beeinträchtigung unterworfen, welche auch zu dem für Stufe 2 des klinisch-neurologischen Scores charakteristischen „Watschelgang“ führt. Um den Gang der Mäuse unter der präsymptomatischen Behandlung möglichst genau zu beobachten und Gangparameter im 
Verlauf der Erkrankung beurteilen zu können, wird ab dem ersten Behandlungstag, dem Lebenstag 50, alle 7 Tage eine videobasierte Analyse des Gangbildes mithilfe des Catwalk XT gait analysis system (Noldus, Wageningen, Niederlande) durchgeführt.

\subsubsection{Durchführung des Catwalk-Tests}

Bei der Durchführung der videobasierten Ganganalyse (Catwalk XT) werden die Mäuse auf einen 4 Zentimeter breiten, sich auf einer durchsichtigen Glasplatte befindlichen und mit undurchsichtigen Plastikscheiben abgetrennten Gang gesetzt. Die Länge des Ganges ist so gewählt, dass bei den gewählten Detektionsparametern und Kriterien für Erfassung eines Laufes mindestens vier Schrittfolgen erfasst werden. Die Mäuse können sich auf diesem Gang frei bewegen, während ihr Gang von unten durch eine Videokamera aufgezeichnet wird. Dabei werden die Fußabdrücke der Mäuse automatisch von der Software CatWalk 10.0 (Noldus, Wageningen, Niederlande) erkannt. Die Detektionsparameter und die Kriterien für Erfassung eines Durchlaufes (run criteria) der Software werden dabei wie folgt eingestellt: „minumum run duration 0,5 s, maximum run duration $30 \mathrm{~s}$, minimum number of compliant runs to acquire 3, maximum allowed speed variation 60\%, intensity threshold 0,30 camera gain 20 “.

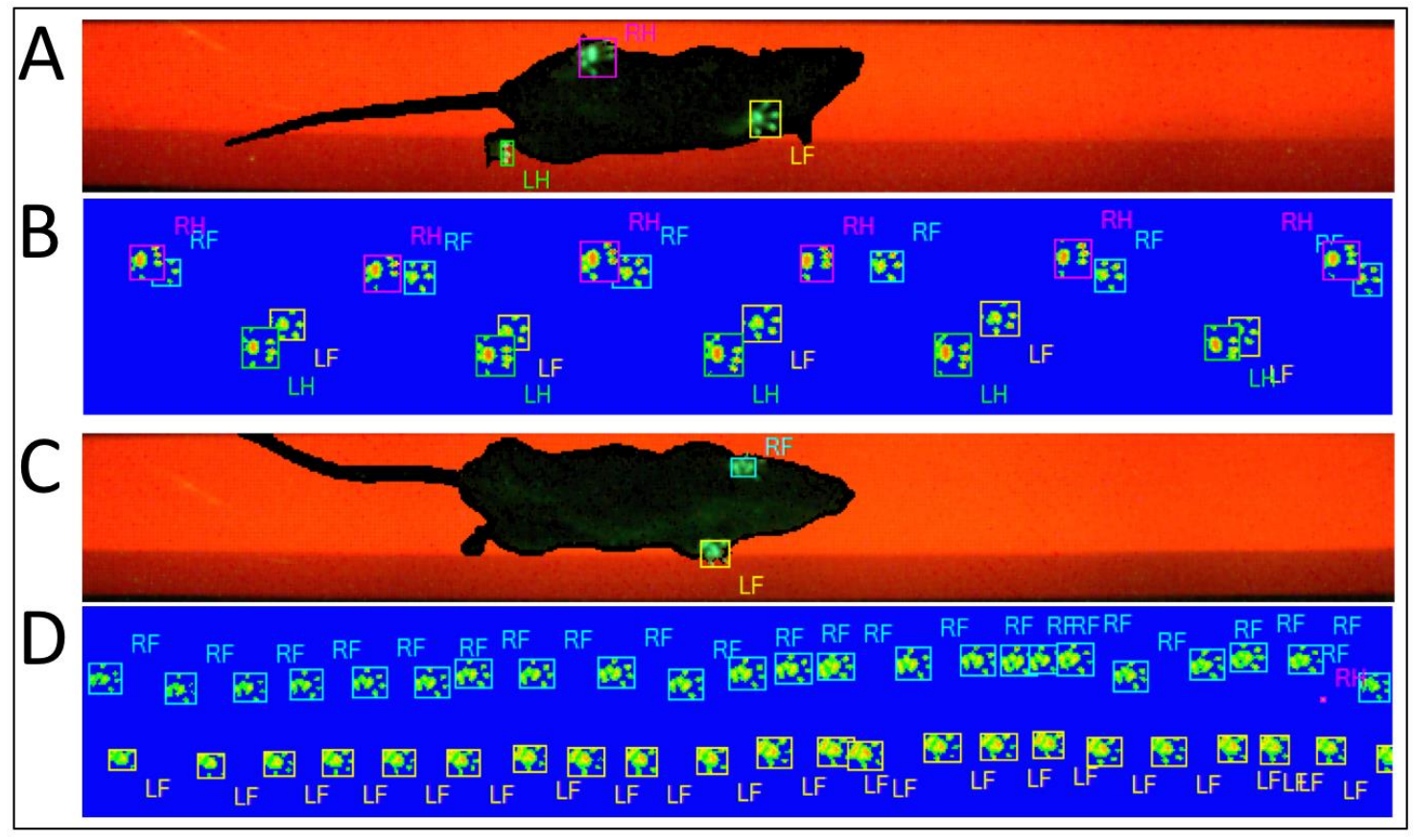

Abbildung 5. Videobasierte Ganganalyse mit dem CatWalk XT gait analysis system

Abgebildet sind das Standbild des Durchlaufes eines Tieres der Wt-Gruppe am Lebenstag 50 (A) mit den dazugehörigen Fußabdrücken (B) und das Standbild des Durchlaufes eines Tieres der Veh-Gruppe am Tag 127 (C) mit den dazugehörigen Fußabdrücken (D). Deutlich erkennbar ist die Paralyse der hinteren Extremitäten, die hinterhergeschleift werden. Bezeichnungen für die Fußabdrücke: RF: right front/rechts vorne, RH: right bind/rechts hinten, LF: left front/links vorne, LH: left hind/links hinten (Quelle: eigenes Bildmaterial). 


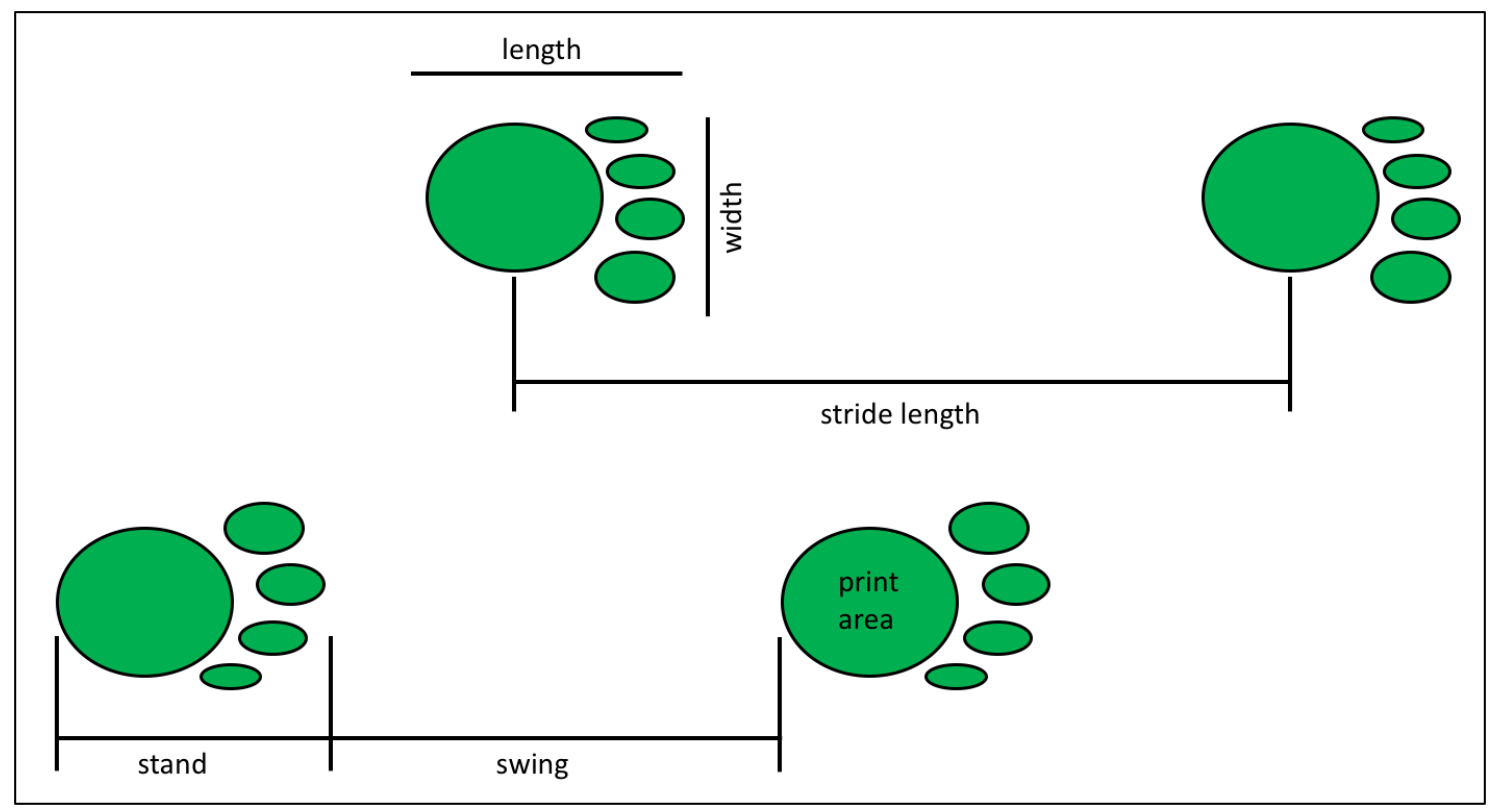

Abbildung 6. Gangparameter der videobasierten Ganganalyse (CatWalk XT)

Abgebildete Gangparameter: stand (Zeit [s] des Bodenkontakts eines Laufs), swing (Zeit [s] ohne Bodenkontakt eines Laufs), length (Auftrittslänge, Länge [cm] des Bodenauftritts eines Laufs), width (Fußbreite, Breite [cm] des Bodenauftritts eines Laufs), print area (Auftrittsfläche [ $\mathrm{cm}^{2}$ ] eines Laufs, gesamte grüne Fläche), stride length (Schrittlänge [cm] eines Laufs). Abbildung modifiziert entnommen aus: Achterkamp et al. 2012, S.100, mit freundlicher Genehmigung von Noldus Information Technology.

Ein Versuchstag beinhaltet dementsprechend drei komplette den Anforderungen entsprechende Durchläufe der Maus. Dabei wird der Mittelwert der erhobenen Parameter an einem Tag gebildet und geht in die Auswertung ein. Es werden im Rahmen der vorliegenden Arbeit nur ausgewählte Parameter ausgewertet und präsentiert. Als Hauptparameter geht in die Auswertung die Durchschnittsgeschwindigkeit (mean average speed) ein. Daneben werden für den rechten Hinter- $(\mathrm{RH})$ und Vorderlauf (RF) die folgenden Parameter ermittelt: Auftrittsfläche (RH/RF print area), Schrittgeschwindigkeit (RH/RF swing speed), Auftrittslänge ( $\mathrm{RH} / \mathrm{RF}$ print length) und Schrittlänge ( $\mathrm{RH} / \mathrm{RF}$ stride length). Die Schrittgeschwindigkeit (swing speed) ergibt sich dabei aus dem Quotienten von Schrittlänge (stride length) und der Zeit ohne Bodenkontakt (swing) eines Laufes (Achterkamp et al. 2012).

\subsubsection{Statistische Auswertung der erhobenen Parameter aus dem Catwalk-Test}

Um auch am letzten ausgewerteten Analysezeitpunkt fünf oder mehr Tiere miteinbeziehen zu können, wird der Zeitraum, in dem die die statistische Analyse erfolgt auf den Zeitaum zwischen Tag 50 und Tag 127 begrenzt. Da der Versuch einmal wöchentlich durchgeführt wird, werden also 12 Analysezeitpunkte in die Auswertung miteinbezogen. Die im Ergebnisteil genannten Werte sind als Mittelwerte $+/$ - Standardfehler zu verstehen, ebenso wie die in Grafiken gezeigten Werte mit Fehlerbalken. 
Die Parameter werden mithilfe einer ANOVA mit Messwiederholungen ausgewertet und signifikante Unterschiede mit dem LSD-Test untersucht. Zudem wird am letzten Messzeitpunkt, dem Tag 127, gesondert ausgewertet und mithilfe einer univarianten ANOVA und LSD-Test auf signifikante Unterschiede geprüft.

\subsubsection{Kognitiver Test (novel object recognition test)}

Neue Erkenntnisse in der ALS-Forschung der letzten Jahre legen eine Verwandtschaft der ALS mit der FTLD nahe. Bei vielen ALS-Patienten kann auch ohne definitive Diagnose eine kognitive Beeinträchtigung im Rahmen der Erkrankung beobachtet werden. Die Pathomechanismen beider Erkrankungen stehen im Verdacht, gleich oder ähnlich zu sein (Brettschneider et al. 2013). Daher erscheint es lohnenswert, die kognitive Entwicklung der Tiere in der Verlaufsstudie genau zu beobachten.

\subsubsection{Durchführung des kognitiven Tests (novel object recognition test)}

Zur Feststellung möglicher Defizite in der kognitiven Entwicklung wird der novel object recognition (NOR)-Test bei den Tieren in der Verlaufsstudie an vier bestimmten Lebenstagen durchgeführt. An Lebenstag 50 beim noch gesunden Tier, an Tag 88 etwa beim mittlerem Symptombeginn unbehandelter Tiere und danach zweimal im Abstand von 14 Tagen, also am Tag 102 und am Tag 116.

Beim NOR-Test wird die Fähigkeit, Objekte wiederzuerkennen, gemessen. Dabei wird die Maus an einem festgelegten Punkt in ein sogenanntes open field mit den Abmessungen $80 \times 80 \mathrm{~cm}$ gesetzt. Sie gewöhnt sich an dieses zuerst für 3 Minuten. Daraufhin werden zwei identische Objekte an zwei festgelegten Orten in der Arena aufgestellt. Sie werden symmetrisch zueinander angeordnet und haben den gleichen Abstand zu den Wänden wie der jeweils andere. Die Maus gewöhnt sich für 5 Minuten an diese Objekte. Nach 10minütiger Erholung im Heimatkäfig wird sie in das open field zurückgesetzt. In der Zwischenzeit wurde eines der bekannten Objekte durch ein anderes, der Maus nie zuvor begegnetes Objekt ersetzt. Das Verhalten der Maus wird nun über 5 Minuten mit einer Videokamera über dem open field aufgezeichnet. Zwischen den einzelnen Schritten wird die Arena immer wieder mit Alkohol gesäubert und vom Kot der Mäuse befreit, um eine Verfälschung der Ergebnisse durch Geruchsspuren oder optische Reize zu vermeiden. Die 5-minütigen Videos werden mithilfe der Computersoftware Ethovision XT 8.5 (Noldus, Wageningen, Niederlande) ausgewertet. Es werden die folgenden Parameter erfasst: Mobilität, Geschwindigkeit und zurückgelegte Distanz. Daneben wird erfasst, über welche Dauer und wie oft der vom Programm erfasste nose-point der Maus sich im Bereich von zwei Zentimeter, um das bekannte und um das unbekannte Objekt befindet. Aus diesen 
Parametern lässt sich der discrimination index errechnen (Antunes und Biala 2012). Der discrimination index errechnet sich aus der Differenz der verbrachten Zeit beim unbekannten minus der beim bekannten Objekt dividiert durch die Gesamtzeit, die bei den Objekten verbracht wurde. Der Mittelwert der Versuchsgruppe wird je Versuchstag ermittelt und geht in die Auswertung ein. Ebenfalls geht die zurückgelegte Distanz (distance moved) in die Auswertung mit ein: als Maß für das Erkundungsverhalten der Maus. In Zusammenschau mit dem discrimination index kann die zurückgelegte Distanz auch als Maß dafür gesehen werden, in welchem Ausmaß die Mobilitätseinschränkung der Mäuse Einfluss auf das Erkundungsverhalten hat, und somit die Kognitionseinschränkung verdeckt.

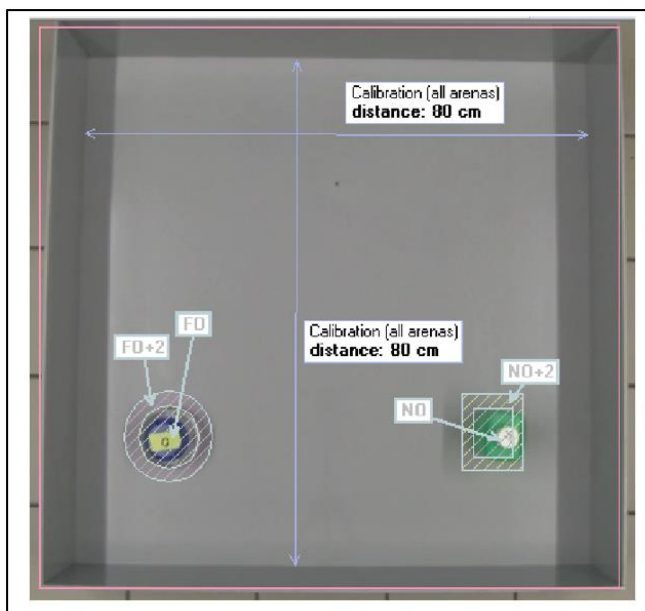

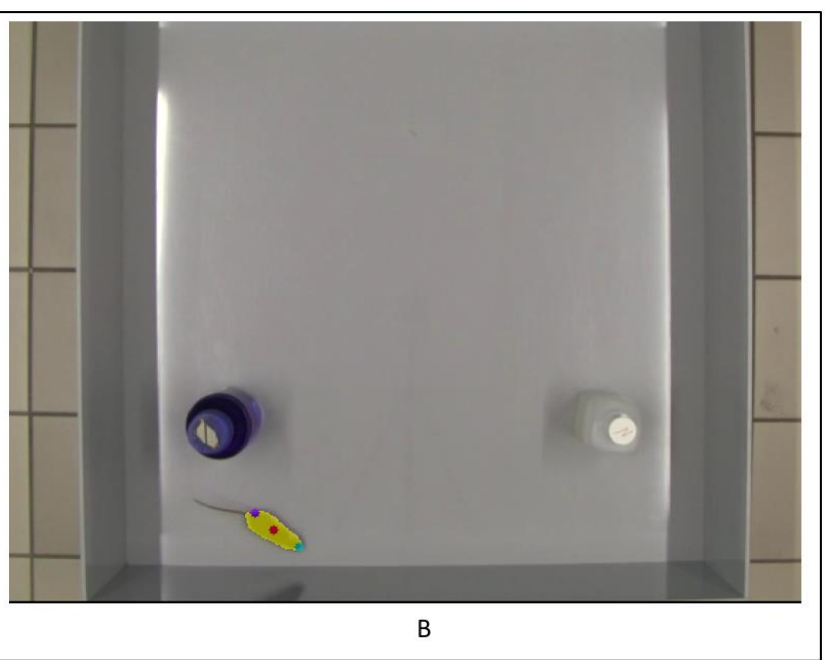

B

Abbildung 7. Versuchsaufbau des kognitiven NOR-Tests

A: Versuchsaufbau bei Aufnahme des 5-minütigen Videos mit definiertem Versuchsareal (rosa Linie), definierten Größenverhältnissen (blaue Linie/“Calibration (all areas) distance: 80 cm“), dem bekannten Objekt/familiar object (FO), dem Bereich $2 \mathrm{~cm}$ um das bekannte Objekt/familiar objekt herum $(\mathrm{FO}+2)$, dem neuen Objekt/ novel object (NO) und dem Bereich $2 \mathrm{~cm}$ um das neue Objekt/novel object herum. B: Versuchsaufbau ohne Beschriftungen mit erfasster Maus (gelber Bereich), Nase „nose point“ (türkis), Körpermitte (rot), Hinterteil (lila) (Quelle: eigenes Bildmaterial).

\subsubsection{Statistische Auswertung des kognitiven Tests}

In die statistische Analyse geht der discrimination index, der aus den Erkundungszeiten der Mäuse an den Objekten durch die Software Ethovision XT 8.5 ermittelt wird, und die zurückgelegte Distanz (distance moved) jedes Tieres mit ein. Pro Versuchstag wird je Gruppe mit der Software Excel der Mittelwert +/- Standardfehler ermittelt und im Text angeben sowie in Säulendiagrammen, wobei der Standardfehler als Fehlerbalken angegeben wird, abgebildet. Die Mittelwerte werden pro Untersuchungstag mittels einer univarianten ANOVA miteinander verglichen und die signifikanten Unterschiede mittels LSD-Test geprüft. Das Signifikanzniveau wird bei $\mathrm{p}<0,05$ festgesetzt und mit einem Sternchen $\left(^{*}\right)$ gekennzeichnet. Zwei Sternchen $\left(^{* *}\right)$ zeigen eine Signifikanz von $\mathrm{p}<0,01$ und drei Sternchen $\left(^{* * *}\right)$ eine Signifikanz von $\mathrm{p}<0,001$ an. 
Miteinander verglichen werden hierbei nur die Ergebnisse eines Untersuchungsstages, da an den verschiedenen Untersuchungstagen jeweils verschiedene Objekte verwendet wurden, an den einzelnen Versuchstagen jeweils aber die identischen, um eine möglichst hohe Vergleichbarkeit eines Versuchstages herzustellen. Da möglicherweise bestimmte Objekte eine höhere Diskriminationsfähigkeit bei Mäusen hervorrufen oder ein erhöhtes Erkundungsverhalten von Mäusen auslösen als andere, sind nur Werte derselben Objekte bzw. desselben Untersuchungstages miteinander gut vergleichbar.

\subsection{Querschnittstudie: Evaluation der spinalen Motoneurone und der Muskelatrophie}

Neben der Verlaufsstudie zur Verhaltensbeobachtung wird eine Querschnittstudie durchgeführt. Evaluiert werden die histologischen Veränderungen der spinalen AlphaMotoneurone und makroskopisch die Atrophie des Musculus gastrocnemius.

Im Zuge der erhofften klinischen Verbesserung ermöglicht diese Querschnittstudie die Beobachtung der medikamentösen Effekte auch auf zellulärer Ebene. Aufgrund vorhergehender histologischer Querschnittstudien zur Anzahl spinaler Motoneurone an Lebenstag 100 in dieser Forschungsgruppe (Günther 2015) ist bekannt, dass der Unterschied zwischen der unbehandelten Veh-Gruppe und der Wt-Gruppe zu diesem Zeitpunkt nicht signifikant ist, obwohl andere Autoren zu diesem Zeitpunkt bereits Degenerationen histologisch nachgewiesen hatten (Fischer et al. 2004). Daher wird ein späterer Analysezeitpunkt, der Lebenstag 120, gewählt, um die weiter fortgeschrittene Degeneration der Motoneurone zu untersuchen. Mögliche Behandlungserfolge sollen so besser erkennbar gemacht werden.

\subsubsection{Perfusion der Versuchstiere}

Am Lebenstag 120 werden alle Versuchstiere, die sich in der Querschnittstudie befinden, euthanasiert, um die Anzahl der Alpha-Motoneurone histologisch und die Atrophie des Musculus gastrocnemius makroskopisch im Rahmen einer Querschnittsstudie zu evaluieren. Die Präparation des Rückenmarks und die immunhistochemische Darstellung der Alpha-Motoneurone wird dabei nach dem in unserer Forschungsgruppe erarbeiteten Protokoll nach Günther et al. (2012) durchgeführt:

Dafür werden die Tiere in einem vorgesehenen Käfig mittels Kohlenstoffdioxidinsufflation euthanasiert. Bei den Tieren wird anschließend der Eintritt des Atemstillstands gewissenhaft untersucht und die Kohlenstoffdioxid-Narkose mittels Schmerzreizen geprüft. Daraufhin werden die Tiere auf einem Perfusionstisch fixiert und die Bauchdecke 
sowie die Brustwand der Tiere mit 70\%igem Alkohol benetzt, um eine Verunreinigung mit Haaren der Tiere zu verhindern und eine leichtere Präparation der Bauchdecke und der Burstwand zu ermöglichen. Diese werden mit einer geraden Schere mit gerader Spitze („Cohan-Vannas Spring Scissors“, Fine Science Tools, Heidelberg, Deutschland) eröffnet, ohne dann freiliegende innere Organe zu verletzen. Das noch schlagende Herz wird vom umliegenden Gewebe und dem Perikard befreit, um die linke Herzkammer mit einer 21G Butterfly-Punktionskanüle (Sarstedt AG \& Co., Nümbrecht, Deutschland) punktieren zu können. Diese wird daraufhin mit einem Schlauchsystem verbunden, durch welches mithilfe eines Infusomaten $100 \mathrm{ml}$ des $4^{\circ} \mathrm{C}$ kalten phosphate-buffered saline (PBS) langsam in den Blutkreislauf des Tieres infundiert werden. Mit einer Schere mit abgewinkelter Spitze (Iris - angled to side, Fine Science Tools, Heidelberg, Deutschland) wird der rechte Herzvorhof eröffnet, um den ansteigenden Druck im Blutkreislauf des Tieres während der Perfusion zu entlasten. Durch dieselbe noch sitzende Kanüle wird dann $100 \mathrm{ml}$ einer $4{ }^{\circ} \mathrm{C}$ kalten 4\%igen Paraformaldehyd-Lösung (PFA) zur Fixierung des gesamten Körpergewebes der Maus geleitet. Die Kontrolle der gelungenen Fixierung erfolgt durch die genaue Beobachtung der einzutretenden Muskelkontraktionen, insbesondere an einer Flexion des gesamten Schwanzes der Maus. Ist dieser komplett starr, sollte auch das gesamte Tier gut perfundiert und das Gewebe fixiert sein.

Anschließend wird die sich in Rückenlage befindliche Maus in Bauchlage mit Nadeln an vorderen und hinteren Extremitäten auf einer Styroporplatte fixiert. Mithilfe eines scharfen Skalpells ("Präzisa Plus", Dahlhausen, Köln, Deutschland) wird die Haut an den unteren Extremitäten in einer transversalen Ebene über den Rumpf verlaufend von der Körperfaszie gelöst und von beiden Seiten kommend bis zum Hinterhaupt über der gesamten Wirbelsäule entfernt. Entsprechend wird mit der Körperfaszie und den darunterliegenden Muskeln verfahren, um die knöchernen Strukturen der Wirbelsäule freizulegen.

\subsubsection{Evaluation der Atrophie des Musculus gastrocnemius}

\subsubsection{Präparation des Musculus gastrocnemius}

Nach der in Kapitel 2.8.1 durchgeführten Präparation wird auch nun auch der Musculus gastrocnemius entsprechend dem Protokoll von Günther (2015) freigelegt und das Gewicht desselben gemessen: Dafür wird die Haut und die Körperfaszie ebenfalls entsprechend an den Hinterläufen entfernt. Durch das Aufsuchen und Durchtrennen der Achillessehne wird der Musculus gastrocnemius vom Gewebe getrennt und an seinem Ursprung vom Körper getrennt. Zur Evaluation der Muskelatrophie wird bereits vor der 
Perfusion das Köpergewicht der Tiere zur Relation bestimmt. Das Muskelgewicht des linken Musculus gastrocnemius wird mithilfe einer Feinwaage bestimmt. In die Auswertung fließen die Daten von jeweils 5 Tieren aus den Behandlungsgruppen und der Veh-Gruppe sowie 10 Tieren aus der Wt-Gruppe ein.

\subsubsection{Statistische Auswertung des Muskelgewichts}

Berechnet wird der prozentuale Anteil des linken Musculus gastrocnemius am Körpergewicht. Dieser wird für die statistische Auswertung herangezogen. Aus den gewonnenen Daten werden Tabellen und Säulendiagramme mit der Software Microsoft Excel Version 2013 hergestellt. Unterschiede zwischen den Behandlungsgruppen werden mithilfe der Software SPSS Version 23 mit einer univarianten ANOVA geprüft. Unterschiede werden mit LSD-Test auf Signifikanz geprüft. Das Signifikanzniveau wird bei $\mathrm{p}<0,05$ festgelegt und mit einem Sternchen $\left(^{*}\right)$ gekennzeichnet. Zwei Sternchen $\left(^{* *}\right)$ zeigen eine Signifikanz von $\mathrm{p}<0,01$ und drei Sternchen $\left.{ }^{(* *}\right)$ eine Signifikanz von $\mathrm{p}<$ 0,001 an.

In der Darstellung der Säulendiagramme wird der Standardfehler als Fehlerbalken gezeigt. Die im Ergebnisteil aufgeführten Werte sind Mittelwerte +/- Standardfehler.

\subsubsection{Rückenmarkspräparation}

Nach der in Kapitel 2.8.1 durchgeführten Präparation wird mit einer feineren Entfernung der autochthonen Rückenmuskulatur von den knöchernen Strukturen der Wirbelsäule fortgefahren. Anschließend erfolgt eine beidseitige Laminektomie mit Beginn am ersten Wirbelbogen. An beiden Seiten werden die Wirbelbögen nacheinander lateral mit einer abgewinkelten Schere (Iris - angled to side, Fine Science Tools, Heidelberg, Deutschland) bis zu den Sakralwirbeln durchtrennt. Dem Schritt für Schritt folgend werden vorsichtig mit einer abgewinkelten Pinzette (HWC 111-10, Hammacher, Solingen, Deutschland) die entstehenden Knochenfragmente vom Durasack angehoben und entfernt. Entstehende Knochenvorstände und überstehende Fragmente werden mit der Pinzette oder der Schere seitwärts abgebrochen oder abgeschnitten.

Der freiliegende Durasack und das darin enthaltene Rückenmark wird mit dem Skalpell auf Höhe des Foramen ovale und der unteren Sakralwirbel durchtrennt und aus dem Wirbelkanal abgehoben. Dabei werden die Spinalnerven mit der Pinzette nach und nach von kranial nach kaudal durchtrennt und so eine saubere Ablösung des Rückenmarks ermöglicht. In einem geeigneten Becherglas erfolgt die Nachfixierung in einer 4\%igen PFA-Lösung bei $4{ }^{\circ} \mathrm{C}$ über eine Stunde. Daraufhin wird dem Rückenmarksgewebe über Nacht in einer 30\%igen Saccharaose-Lösung (AppliChem GmbH, Darmstadt, 
Deutschland) Wasser entzogen. Die ausreichende Dehydrierung ist durch das Absinken der Gewebeproben auf den Boden des Gefäßes zu kontrollieren, während diese anfangs an der Oberfläche schwimmen.

Auf Höhe der Intumescencia lumbalis (L3-L6) wird ein circa 0,5 cm langes Stück mit einem Skalpell herausgeschnitten und in eine Kryomatrix eingebettet (Shandon Cyromatrix, Thermo Fisher Scientific Inc, Kalamazoo, Michigan, USA). Dabei wird auf dem Gefäß, das zur Einbettung dient, das kaudale Ende des eingebetteten Rückenmarks markiert. Die eingebetteten Proben lagern fortan bei $-80{ }^{\circ} \mathrm{C}$ zur weiteren Verarbeitung. Das restliche Gewebe wird in Alufolie verpackt und ebenfalls bei $-80{ }^{\circ} \mathrm{C}$ für mögliche Kontrollversuche gelagert.

\subsubsection{Gewebeaufbereitung}

Aus den eingebetteten Gewebeproben werden mit einem Kryostat von kaudal ausgehend $20 \mu \mathrm{m}$ dünne transversale Gefrierschnitte gefertigt. Dabei werden, um das Rückenmark im richtigen Winkel zu schneiden bei jeder Gewebeprobe genau 10 Probeschnitte durchgeführt, sodass alle Gewebeproben an möglichst derselben Stelle im Rückenmark untersucht werden. Insgesamt werden 25 Schnitte nacheinander in folgender Ordnung auf 5 Objektträger aufgetragen: Die ersten fünf Schnitte werden nacheinander an die erste Position je eines Objektträgers platziert, dann folgend werden die nächsten Schnitte in ebenjener Ordnung auf die nächsten Positionen der Objektträger aufgebracht, sodass die fünf auf jedem Objektträger liegenden Schnitte einen Gewebeabstand von je $100 \mu \mathrm{m}$ zueinander haben. Diese werden bis zur weiteren Verwendung bei $-20{ }^{\circ} \mathrm{C}$ gelagert.

\subsubsection{Immunhistochemische Darstellung der Alpha-Motoneurone}

Zur Färbung der Motoneurone, die zu den cholinergen Neuronen zählen, wird ein Antikörper gegen die Cholin-Acetyl-Transferase (ChAT) genutzt. Acetylcholin dient den Motoneuronen als Neurotransmitter und ist unerlässlich für die Erregungsweiterleitung des motorischen Systems. Der Wiederaufbau des im synaptischen Spalt durch die Acetylcholinesterase gespaltenen und die Synthese neuen Acetylcholins erfolgt durch das angefärbte Enzym im Motoneuron. Daher liegt es in großen Mengen vor allem in cholinergen Neuronen vor und ist ein etablierter Marker für Motoneurone im Rückenmark. Es werden für die im Folgenden durchgeführte immunhistochemische Färbung der AlphaMotoneurone die Objektträger der entsprechenden Tiere mit der gleichen Nummerierung ausgewählt, um eine möglichst hohe Vergleichbarkeit der Gewebeproben durch die möglichst sorgfältige Angleichung der Schnitthöhe im Rückenmark zu erzielen. 
Die ausgewählten Objektträger werden, nachdem sie aus dem Gefrierschrank entnommen wurden, zunächst für 30 Minuten in einem Inkubationsschrank getrocknet. Auf einem Schwenktisch werden die Objektträger in einer Küvette, die mit PBS gefüllt ist, rehydriert. Um die Gewebeproben optimal für die Behandlung mit Antikörpern vorzubereiten und deren Bindungsstellen im Gewebe freizulegen, werden die Objektträger im Rahmen des „Retrieval“ mit einer $10 \mathrm{mM}$ Zitronenlösung in der Küvette in einem $80{ }^{\circ} \mathrm{C}$ heißem Wasserbad für 30 Minuten behandelt. Daraufhin werden die Objektträger in der Küvette auf Raumtemperatur wieder abgekühlt und in einer mit PBS gefüllten Küvette für fünf Minuten gewaschen. Damit in den folgenden Schritten ein Verlaufen der auf den Objektträger aufgebrachten Flüssigkeiten möglichst verhindert wird, werden die Proben mit einem Konturstift (Super PAP Pen Liquid Blocker, Science Services GmbH, München, Deutschland) umrandet.

Als erstes werden auf die Proben nun eine Phosphatpufferlösung mit 5\% NDS (Normal Donkey Serum, Dianova GmbH, Hamburg, Deutschland) und 0,2\% TritonX100 (T-8787, Lot 46H2616, Sigma-Aldrich, St. Louis, Missouri, USA) versehen und für eine Stunde bei Raumtemperatur gelagert, um eine unspezifische Reaktion der Antikörper im nächsten Schritt zu verhindern.

Nachdem die Lösung wieder entfernt worden ist, wird auf die Proben eine Phosphatpufferlösung mit dem Primärantikörper Anti-ChAT (Choline Acetyltransferase, goat, AB144 P, Merck Millipore, Billerica, Massachusetts, USA) in einem Verdünnungsverhältnis von 1:100 aufgetragen. Dieser wird bei $4{ }^{\circ} \mathrm{C}$ über Nacht inkubiert. Anschließend werden die Präparate dreimal für 15 Minuten mit jeweils ausgewechselter Phosphatpufferlösung auf einem Schwenktisch gewaschen, um dann den in einer Phosphatpufferlösung in einer Verdünnung von 1:300 gelösten Sekundärantikörper Cy3 (donkey anti goat, Dianova GmbH, Hamburg, Deutschland) aufzutragen und im Dunkeln in einer Plastikbox eine Stunde lang bei Raumtemperatur zu inkubieren. Danach werden die Objektträger wie zuvor dreimal für 15 Minuten gewaschen. Um beim späteren Mikroskopieren Zellen identifizieren zu können, wird für 10 Minuten eine DAPI-Lösung (4,6-diamidino-2-phenylindole, Sigma-aldrich, St. Louis, Missouri, USA) in einer Verdünnung von 1:5000 aufgetragen. Nachdem von dieser die Zellkerne angefärbt wurden, werden die Objektträger wiederholt, diesmal dreimal 5 Minuten, gewaschen. Als letzter Schritt erfolgt das Eindeckeln mit Mowiol (Hoechst, Frankfurt, Germany) und einem Deckgläschen. 


\subsubsection{Mikroskopie und Auswertung der Motoneurone}

Es befinden sich auf jedem Objektträger durch die Anordnung der Schnitte, wie in Kapitel 2.8.3 beschrieben, jeweils 5 Rückenmarksquerschnitte mit einem jeweiligen Gewebsabstand von $100 \mu \mathrm{m}$ zum benachbarten Querschnitt. Es werden pro Behandlungsgruppe, aus der Veh-Gruppe und aus der Wt-Gruppe jeweils 5 Tiere untersucht. Die Gesamtzahl der Motoneurone in einem Rückenmarksvorderhorn wird für jedes der 10 pro Tier mikroskopierten Vorderhörner dokumentiert und in die Auswertung einbezogen. Dazu wird ein Axioplan Fluoreszenzmikroskop angewandt. Die Darstellung der wie in Kapitel 2.8.4 gefärbten Motoneurone gelingt unter 10-facher Vergrößerung durch den Cy3 Filter (rot) und die Darstellung der Zellkerne entsprechend durch einen DAPI Filter (blau). Als ein Alpha-Motoneuron gilt bei der Auswertung eine ChAT-positive Zelle mit einem erkennbar im Zellkörper befindlichem Zellkern. Die Auszählung wird vom Untersucher beim Mikroskopieren dokumentiert und die mikroskopierten Vorderhörner als Bilddateien über einen am Mikroskop angeschlossenen Computer gesichert. In die Auswertung geht der Mittelwert der Motoneurone pro Vorderhorn ein.

\subsubsection{Statistische Auswertung der histologisch gewonnenen Daten}

Aus den immhistochemisch gewonnenen Daten werden Tabellen und Säulendiagramme mit der Software Microsof Excle Version 2013 hergestellt. Unterschiede zwischen den Behandlungsgruppen werden mit der Software SPSS Version 23 mit einer univarianten ANOVA geprüft. Unterschiede werden mit LSD-Test auf Signifikanz geprüft. Das Signifikanzniveau wird bei $\mathrm{p}<0,05$ festgesetzt und mit einem Sternchen $\left(^{*}\right)$ gekennzeichnet. Zwei Sternchen $(* *)$ zeigen eine Signifikanz von $\mathrm{p}<0,01$ und drei Sternchen $(* * *)$ eine Signifikanz von $\mathrm{p}<0,001$ an.

In der Darstellung der Säulendiagramme wird der Standardfehler als Fehlerbalken gezeigt. Die im Ergebnisteil aufgeführten Werte sind als Mittelwerte +/- Standardfehler zu verstehen. 


\section{Ergebnisse}

\subsection{Verlaufsstudie unter präsymptomatischer Behandlung mit Fasudil und Riluzol ab Lebenstag 50}

Es wird eine Verlaufsstudie zur verhaltensbiologischen Evaluation unter präsymptomatischer Behandlung ab Lebenstag 50 mit Fasudil in zwei Dosierungen und Riluzol, sowie einer Kombination beider Substanzen durchgeführt. Dabei werden pro Gruppe transgener Mäuse (Veh, Ril30, Fas10, Fas30, Fas10Ril30) zwölf Tiere untersucht. Jedoch muss ein Tier in der Fas10-Gruppe nachträglich zensiert werden, da es keine Symptome der Erkrankung entwickelte und bei einer erneuten Kontrolle als ein falsch positiv genotypisiertes Tier identifiziert wurde. In der Gruppe der gesunden Kontrolle (WtGruppe) werden 7 Tiere untersucht. Es wird in der Verlaufsstudie eine klinische Analyse des Überlebens, des Krankheitsverlaufs und des Körpergewichts durchgeführt. Es wird eine Analyse der motorischen Performance mithilfe des Drehwalzentests (Rotarod) und mithilfe einer videobasierten Ganganalyse (Catwalk XT) durchgeführt. Zudem wird eine Analyse der kognitiven Leistung (novel object recognition (NOR)-Test) durchgeführt.

\subsubsection{Klinische Analyse: Überleben, Krankheitsverlauf und Körpergewicht}

\subsubsection{1 Überleben}

Das mittlere Überleben der Veh-Gruppe beträgt 136,5 +/- 2,7 Tage, in der Ril30-Gruppe 138,4 +/- 3,3 Tage, in der Fas10-Gruppe 137,3 +/- 3,25 Tage, in der Fas30-Gruppe 141,92 +/- 3,5 Tage, in der Fas10Ril30-Gruppe 137,5 +/- 3 Tage.

Nach statistischer Auswertung mit Kaplan-Meier-Schätzer und Analyse mit dem log-RankTest ergeben sich keine signifikanten Unterschiede zwischen den Behandlungsgruppen. 


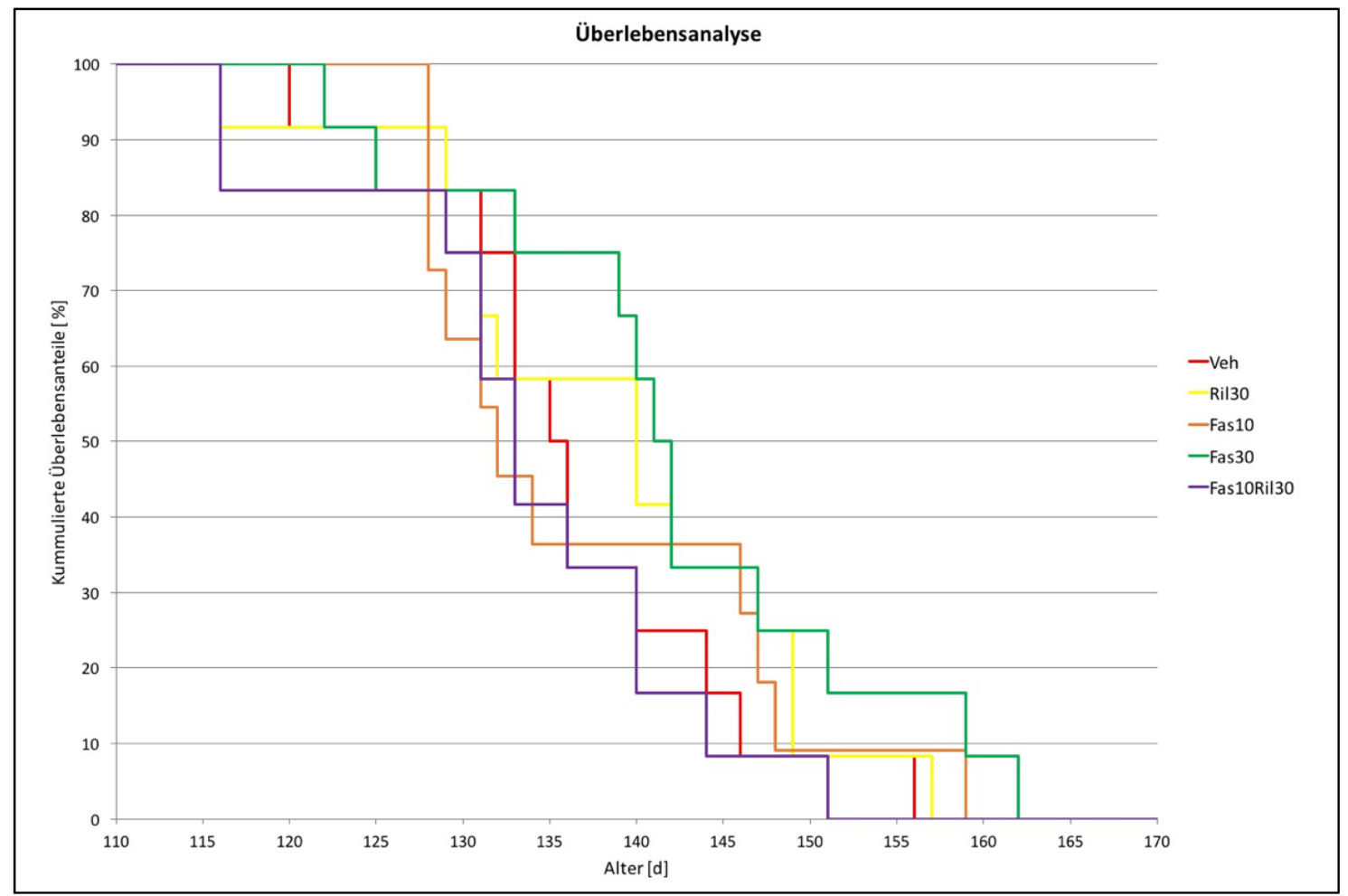

Abbildung 8. Überlebensanalyse der Behandlungsgruppen

Untersucht werden SOD1G93A-transgene weibliche Tiere, die mit Wasser (Veh, n=12), 30 mg/kg KG Riluzol (Ril30, n=12), $10 \mathrm{mg} / \mathrm{kg} \mathrm{KG} \mathrm{Fasudil} \mathrm{(Fas10,} \mathrm{n=11),} 30 \mathrm{mg} / \mathrm{kg}$ KG Fasudil (Fas30, n=12) und $10 \mathrm{mg} / \mathrm{kg}$ KG Fasudil in Kombination mit $30 \mathrm{mg} / \mathrm{kg} \mathrm{KG}$ Riluzol (Fas10Ril30, n=12) ab dem Lebenstag 50 behandelt werden. Es ergeben sich keine signifikanten Unterschiede im log-Rank-Test.

\subsubsection{Gewichtsanalyse}

Jedes Tier wird bis zum Versuchsabbruch sowohl klinisch durch die Messung des Körpergewichts und Erhebung des klinisch-neurologischen Scores als auch motorisch durch den Drehwalzenversuch beobachtet. Um in jeder Gruppe auch an den letzten Untersuchungstagen mindestens 10 Tiere zu untersuchen, wird das Ende des Analysezeitraums auf den Tag 123 gesetzt. In diesem Zeitraum wird das Körpergewicht zweimal die Woche gemessen, woraus sich 22 Messzeitpunkte ergeben (siehe Tabelle 5).

Die ANOVA mit Messwiederholungen (Grafik siehe Abbildung 12) zeigt keine signifikanten Unterschiede zwischen der Veh-Gruppe und den Behandlungsgruppen (Ril30, Fas10, Fas30 und Fas10Ril30). 


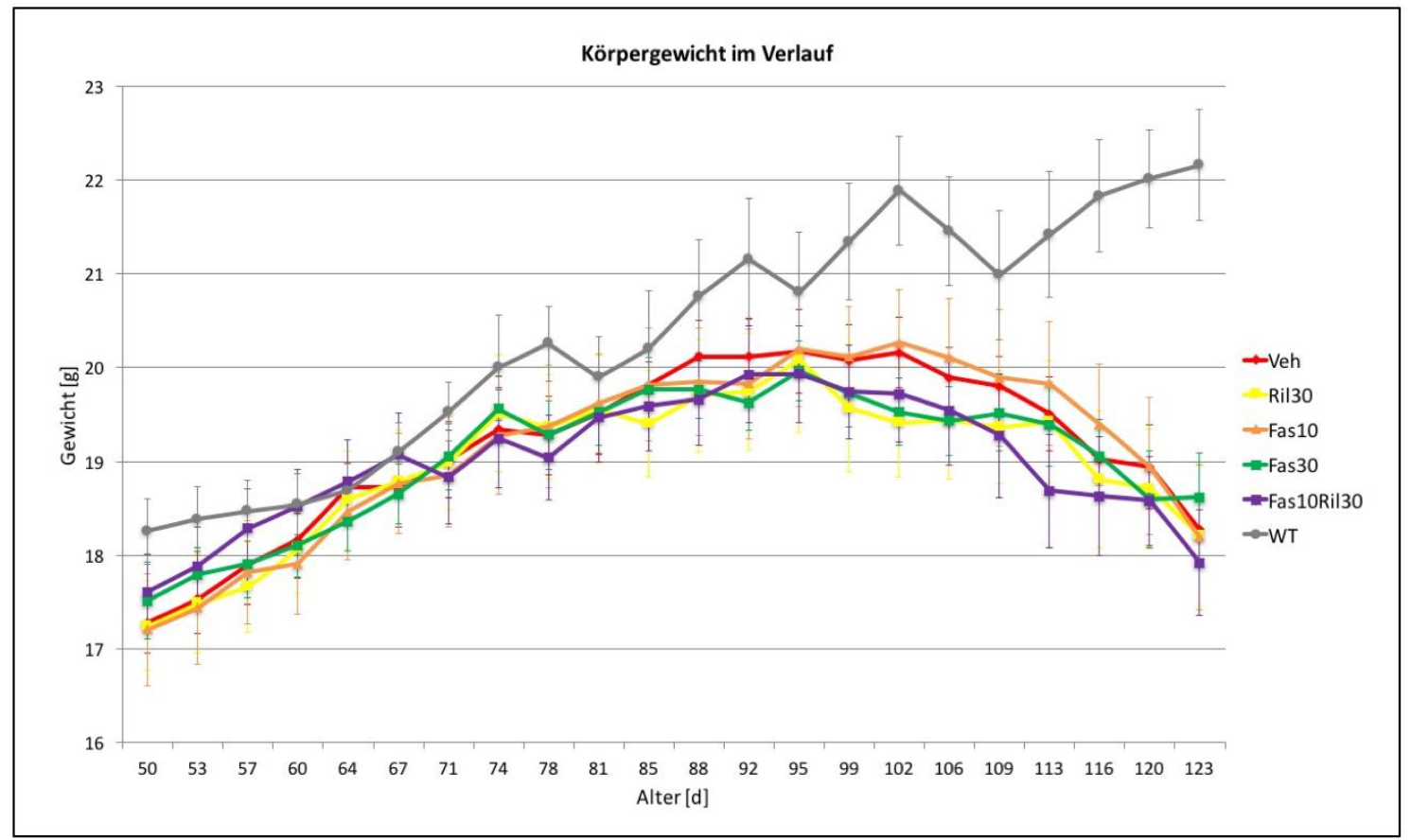

Abbildung 9. Körpergewicht im Verlauf

Untersucht werden SOD1G93A-transgene weibliche Tiere, die mit Wasser (Veh), $30 \mathrm{mg} / \mathrm{kg}$ KG Riluzol (Ril30), 10 mg/kg KG Fasudil (Fas10), 30 mg/kg KG Fasudil (Fas30) und 10 mg/kg KG Fasudil in Kombination mit 30 mg/kg KG Riluzol (Fas10Ril30) ab dem Lebenstag 50 behandelt werden. Als gesunde Kontrollgruppe werden 7 SOD1-Wildtyp-Tiere (WT) untersucht. Anzahl der untersuchten transgenen Tiere je Versuchstag und Gruppe: siehe Tabelle 5. Angegeben sind Mittelwerte +/- Stdf.

Die maximale Gewichtszunahme im Vergleich zum Anfangsgewicht an Lebenstag 50 beträgt in der Veh-Gruppe im Mittel 16,87\%. Sie wird mit im Mittel 20,175 g (+/-0,44 g) am Lebenstag 95 erreicht. Die Ril30-Gruppe erreicht diesen Wert ebenfalls am Lebenstag 95 mit im Mittel 16,27\% und 20,08 g (+/- 0,77). Die Fas10-Gruppe dagegen erreicht ihr mittleres Maximalgewicht erst am Lebenstag 102 mit einer mittleren maximalen Gewichtszunahme von 18,27\% mit 20,26 g (+/-0,56 g). Die Fas30-Gruppe erreicht ihr mittleres Maximalgewicht von 19,97 g (+/- 0,32 g) am Lebenstag 95, was einer Zunahme von 14,48\% entspricht. Bei der Fas10Ril30-Gruppe wird das mittlere Maximalgewicht von 19,93 g (+/- 0,52 g) ebenfalls am Lebenstag 95 erreicht, was einer Zunahme von 13,32\% entspricht.

Der Verlauf des Gewichtsverlustes (Abbildung 13) wird sowohl über den Zeitraum von Tag 50 bis Tag 123 als auch ab Tag 95 bis Tag 123 für alle Gruppen mithilfe einer ANOVA mit Messwiederholungen verglichen und mit dem LSD-Test auf Signifikanz geprüft. Es zeigen sich keine signifikanten Unterschiede zwischen der Veh-Gruppe und den Behandlungsgruppen (Ril30, Fas10, Fas30, Fas10Ril30). 


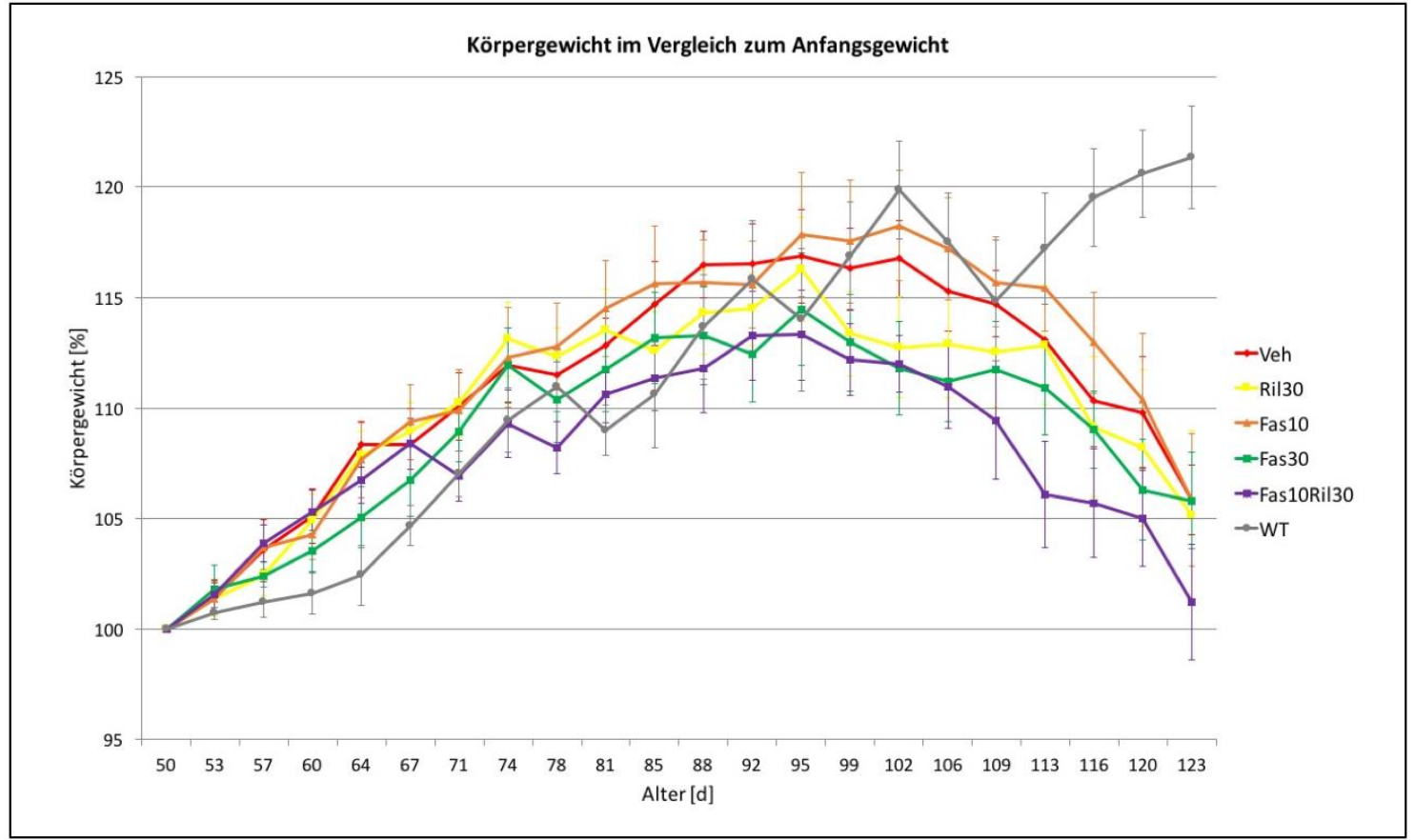

Abbildung 10. Körpergewicht im Vergleich zum Anfangsgewicht

Untersucht werden SOD1G93A-transgene weibliche Tiere, die mit Wasser (Veh), $30 \mathrm{mg} / \mathrm{kg}$ KG Riluzol (Ril30), 10 $\mathrm{mg} / \mathrm{kg} \mathrm{KG}$ Fasudil (Fas10), $30 \mathrm{mg} / \mathrm{kg}$ KG Fasudil (Fas30) und $10 \mathrm{mg} / \mathrm{kg} \mathrm{KG}$ Fasudil in Kombination mit $30 \mathrm{mg} / \mathrm{kg} \mathrm{KG}$ Riluzol (Fas10Ril30) ab dem Lebenstag 50 behandelt werden. Als gesunde Kontrollgruppe werden 7 SOD1-Wildtyp-Tiere (WT) untersucht. Anzahl der untersuchten transgenen Tiere je Versuchstag und Gruppe: siehe Tabelle 5. Angegeben sind Mittelwerte +/- Stdf.

\subsubsection{Krankheitsverlauf}

Der mittlere Krankheitsbeginn, definiert als ein erstmalig festgestellter Score 3 (Tremor der Hinterläufe, siehe Kapitel 2.7.1.1), zeigt sich bei der unbehandelten Veh-Gruppe bei 103,5 +/- 3,02 Tagen, bei der Ril30-Gruppe nach 106,17 +/- 3,81 Tagen, bei der Fas10-Gruppe nach 89,91 +/- 2,24 Tagen, bei der Fas30-Gruppe nach 103,67 +/- 3,83 Tage und bei der Fas10/Ril30-Gruppe nach 98,83 +/- 2,04 Tagen.

In der statistischen Analyse des mittleren Krankheitsbeginns zeigt sich im LSD-Test ein signifikant früherer Krankheitsbeginn in der mit Fasudil $10 \mathrm{mg} / \mathrm{kg} \mathrm{KG}$ behandelten Gruppe im Gegensatz zur Veh-Gruppe und ebenfalls zu den Gruppen Ril30 und Fas30. Nicht signifikant $(p=0,053)$ ist der Unterschied zur Fas10Ril30-Gruppe. 


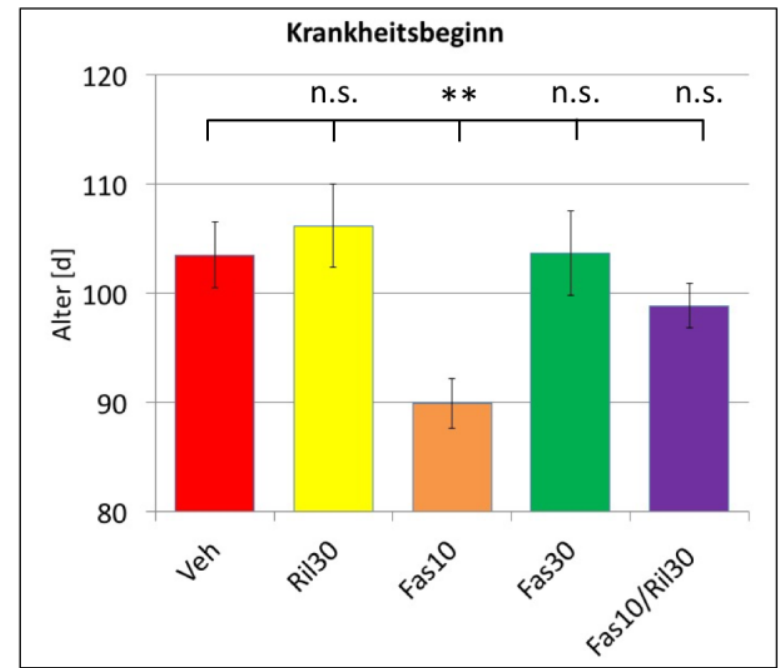

Abbildung 11. Krankheitsbeginn

Untersucht werden SOD1G93A-transgene weibliche Tiere, die mit Wasser (Veh, n=12), 30 mg/kg KG Riluzol (Ril30, n=12), $10 \mathrm{mg} / \mathrm{kg}$ KG Fasudil (Fas10, n=11), $30 \mathrm{mg} / \mathrm{kg} \mathrm{KG} \mathrm{Fasudil} \mathrm{(Fas30,} \mathrm{n=12)} \mathrm{und} 10 \mathrm{mg} / \mathrm{kg}$ KG Fasudil in Kombination mit $30 \mathrm{mg} / \mathrm{kg}$ KG Riluzol (Fas10Ril30, n=12) ab dem Lebenstag 50 behandelt werden. Als gesunde Kontrollgruppe werden 7 SOD1-Wildtyp-Tiere (WT) untersucht. Signifikante Unterschiede zwischen den Gruppen sind bei $\mathrm{p}<0,01 \mathrm{mit}\left({ }^{* *}\right)$ markiert, n. s. nicht signifikant. Angegeben sind Mittelwerte +/- Stdf.

Den Score 2 (gestörtes Gangbild, siehe Kapitel 2.7.1.1) erreichen die Gruppen im Mittel an folgenden Tagen: Veh-Gruppe 123,83 +/- 1,95 Tage, Ril30-Gruppe 126,42 +/- 2,88 Tage, Fas10-Gruppe 124,45 +/- 1,95 Tage, Fas30-Gruppe 128,25 +/- 2,54 Tage, Fas10Ril30Gruppe $122+/-2,53$ Tage.

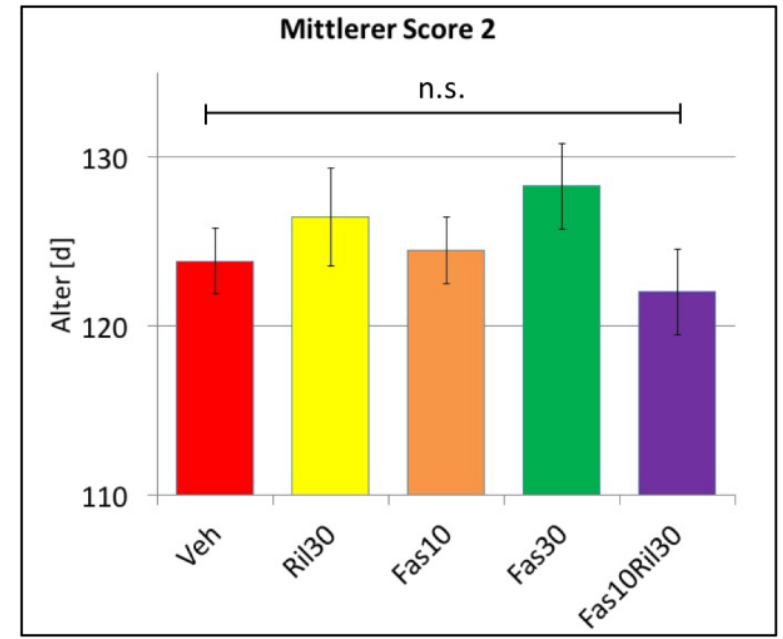

Abbildung 12. Mittlerer Score 2

Untersucht werden SOD1G93A-transgene weibliche Tiere, die mit Wasser (Veh, n=12), 30 mg/kg KG Riluzol (Ril30, n=12), $10 \mathrm{mg} / \mathrm{kg} \mathrm{KG} \mathrm{Fasudil} \mathrm{(Fas10,} \mathrm{n=11),} 30 \mathrm{mg} / \mathrm{kg} \mathrm{KG} \mathrm{Fasudil} \mathrm{(Fas30,} \mathrm{n=12)} \mathrm{und} 10 \mathrm{mg} / \mathrm{kg} \mathrm{KG}$ Fasudil in Kombination mit $30 \mathrm{mg} / \mathrm{kg}$ KG Riluzol (Fas10Ril30, n=12) ab dem Lebenstag 50 behandelt werden. Als gesunde Kontrollgruppe werden 7 SOD1-Wildtyp-Tiere (WT) untersucht. Die Unterschiede zwischen den Gruppen sind nicht signifikant (n. s.). Angegeben sind Mittelwerte +/- Stdf.

Einen mittleren Score 1 (Lähmung einer hinteren Extremität, siehe Kapitel 2.7.1.1) erreichen die Gruppen an folgenden Tagen: Veh-Gruppe bei 130,75 +/- 2,51 Tagen, Ril30- 
Gruppe bei 132,83 +/- 3,37 Tagen, Fas10-Gruppe bei 133,36 +/- 2,99 Tagen, Fas30Gruppe bei 127,22 +/- 3,25 Tagen, Fas10Ril30-Gruppe bei 129,58 +/- 2,89 Tagen.

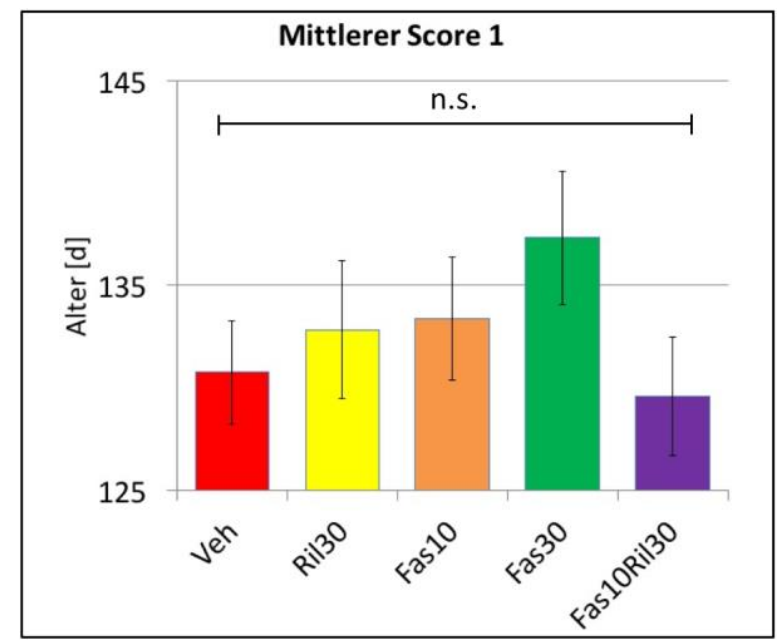

Abbildung 13. Mittlerer Score 1

Untersucht werden SOD1G93A-transgene weibliche Tiere, die mit Wasser (Veh, n=12), $30 \mathrm{mg} / \mathrm{kg} \mathrm{KG}$ Riluzol (Ril30, n=12), $10 \mathrm{mg} / \mathrm{kg} \mathrm{KG} \mathrm{Fasudil} \mathrm{(Fas10,} \mathrm{n=11),} 30 \mathrm{mg} / \mathrm{kg}$ KG Fasudil (Fas30, n=12) und $10 \mathrm{mg} / \mathrm{kg} \mathrm{KG}$ Fasudil in Kombination mit $30 \mathrm{mg} / \mathrm{kg}$ KG Riluzol (Fas10Ril30, n=12) ab dem Lebenstag 50 behandelt werden. Als gesunde Kontrollgruppe werden 7 SOD1-Wildtyp-Tiere (WT) untersucht. Die Unterschiede zwischen den Gruppen sind nicht signifikant (n. s.). Angegeben sind Mittelwerte +/- Stdf.

Einen mittleren Score 0 (Versuchsabbruch, siehe Kapitel 2.7.1.1) erreichen die Gruppen an folgenden Tagen: Veh-Gruppe bei 136,5 +/- 2,56 Tagen, Ril30-Gruppe bei 138,42 +/- 3,3 Tagen, Fas10-Gruppe bei 137,27 +/- 3,25 Tagen, Fas30-Gruppe bei 141,92 +/- 3,46 Tagen, Fas10Ril30-Gruppe bei 133,33 +/- 2,96 Tagen.

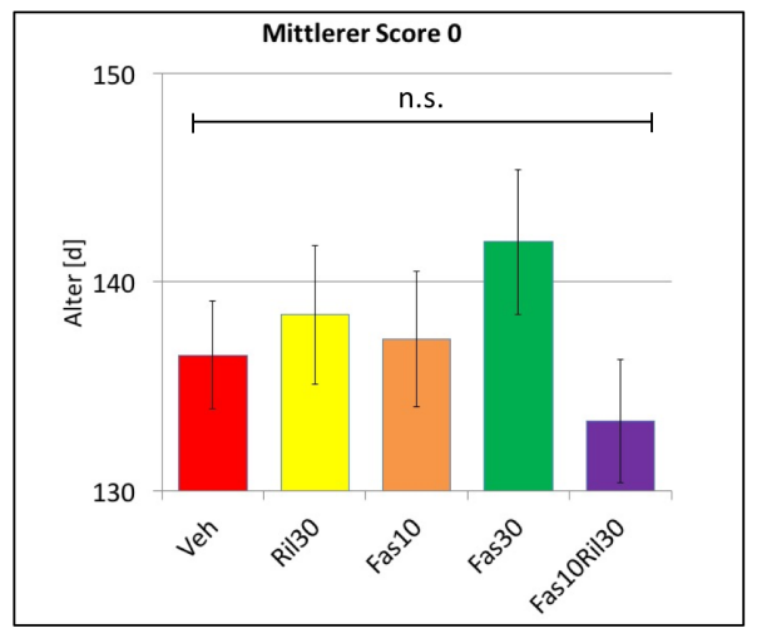

Abbildung 14. Mittlerer Score 0

Untersucht werden SOD1G93A-transgene weibliche Tiere, die mit Wasser (Veh, n=12), 30 mg/kg KG Riluzol (Ril30, n=12), $10 \mathrm{mg} / \mathrm{kg} \mathrm{KG} \mathrm{Fasudil} \mathrm{(Fas10,} \mathrm{n=11),} 30 \mathrm{mg} / \mathrm{kg} \mathrm{KG} \mathrm{Fasudil} \mathrm{(Fas30,} \mathrm{n=12)} \mathrm{und} 10 \mathrm{mg} / \mathrm{kg} \mathrm{KG}$ Fasudil in Kombination mit $30 \mathrm{mg} / \mathrm{kg}$ KG Riluzol (Fas10Ril30, n=12) ab dem Lebenstag 50 behandelt werden. Als gesunde Kontrollgruppe werden 7 SOD1-Wildtyp-Tiere (WT) untersucht. Die Unterschiede zwischen den Gruppen sind nicht signifikant (n. s.). Angegeben sind Mittelwerte +/- Stdf. 
Aus den Daten zum Krankheitsverlauf lässt sich die Gesamtdauer der Erkrankung errechnen. Sie ergibt sich aus der Differenz zwischen dem Todeszeitpunkt und Symptombeginn.

Die Krankheitsdauer beträgt in den Gruppen: Veh-Gruppe 33 +/- 4,9 Tage, Ril30-Gruppe 32,25 +/- 5,16 Tage, Fas10-Gruppe 47,36 +/- 4,09 Tage, Fas30-Gruppe 38,25 +/- 4,47 Tage, Fas10Ril30-Gruppe bei 34,5 +/- 3,98 Tage.

Die Erkrankungsdauer der Fas10-Gruppe ist signifikant länger als die der Veh-Gruppe und die der Ril30-Gruppe. Alle anderen Unterschiede zwischen den Gruppen sind nicht signifikant.

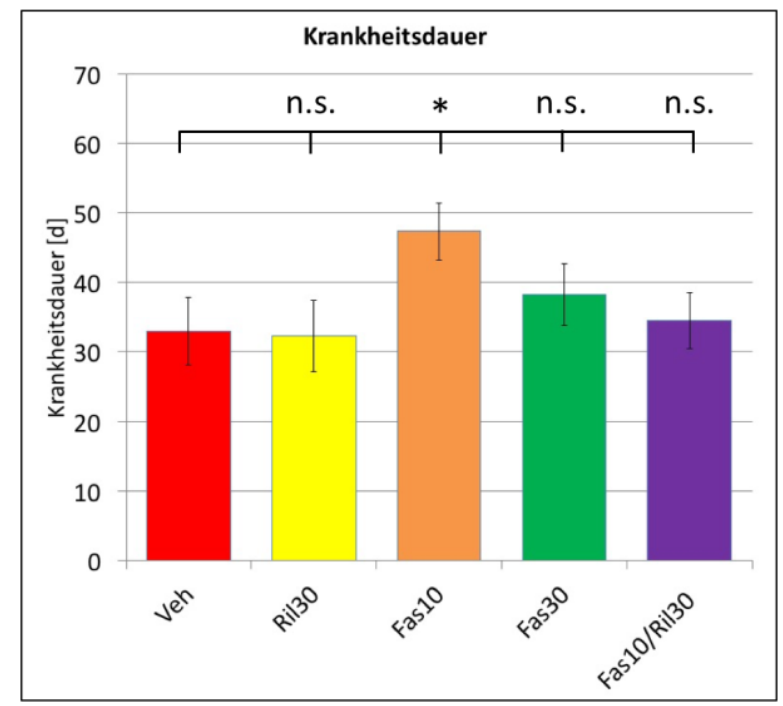

Abbildung 15. Krankheitsdauer

Untersucht werden SOD1G93A-transgene weibliche Tiere, die mit Wasser (Veh, n=12), 30 mg/kg KG Riluzol (Ril30, n=12), $10 \mathrm{mg} / \mathrm{kg} \mathrm{KG} \mathrm{Fasudil} \mathrm{(Fas10,} \mathrm{n=11),} 30 \mathrm{mg} / \mathrm{kg} \mathrm{KG} \mathrm{Fasudil} \mathrm{(Fas30,} \mathrm{n=12)} \mathrm{und} 10 \mathrm{mg} / \mathrm{kg} \mathrm{KG} \mathrm{Fasudil} \mathrm{in}$ Kombination mit $30 \mathrm{mg} / \mathrm{kg}$ KG Riluzol (Fas10Ril30, n=12) ab dem Lebenstag 50 behandelt werden. Als gesunde Kontrollgruppe werden 7 SOD1-Wildtyp-Tiere (WT) untersucht. Signifikante Unterschiede zwischen den Gruppen sind bei $\mathrm{p}<0,05$ mit $\left(^{*}\right)$ markiert, n. s. nicht signifikant. Angegeben sind Mittelwerte +/- Stdf.

\subsubsection{Motorische Verhaltensanalyse (Drehwalzentest)}

Jedes Tier wird bis zum Versuchsabbruch sowohl klinisch durch die Messung des Körpergewichts und Erhebung des klinisch-neurologischen Scores als auch motorisch durch den Drehwalzenversuch beobachtet. Für die Auswertung gilt: Um auch am letzten Untersuchungszeitpunkt pro Gruppe zehn oder mehr Individuen mit einzubeziehen, wird wie bei der Auswertung des Körpergewichts ein Untersuchungszeitraum von Tag 50 bis Tag 123 festgelegt (Anzahl untersuchter Tiere je Gruppe und Versuchstage: siehe Tabelle 5). 


\begin{tabular}{|c|c|c|c|c|c|c|c|c|c|c|c|c|}
\hline $\begin{array}{l}\text { Untersuchungs- } \\
\text { tag }\end{array}$ & 50 & 53 & 57 & 60 & 64 & 67 & 71 & 74 & 78 & 81 & 85 & 88 \\
\hline Veh & 12 & 12 & 12 & 12 & 12 & 12 & 12 & 12 & 12 & 12 & 12 & 12 \\
\hline Ril30 & 12 & 12 & 12 & 12 & 12 & 12 & 12 & 12 & 12 & 12 & 12 & 12 \\
\hline Fas10 & 11 & 11 & 11 & 11 & 11 & 11 & 11 & 11 & 11 & 11 & 11 & 11 \\
\hline Fas30 & 12 & 12 & 12 & 12 & 12 & 12 & 12 & 12 & 12 & 12 & 12 & 12 \\
\hline Fas10Ril30 & 12 & 12 & 12 & 12 & 12 & 12 & 12 & 12 & 12 & 12 & 12 & 12 \\
\hline Wt & 7 & 7 & 7 & 7 & 7 & 7 & 7 & 7 & 7 & 7 & 7 & 7 \\
\hline $\begin{array}{l}\text { Untersuchungs- } \\
\text { tag }\end{array}$ & 92 & 95 & 99 & 102 & 106 & 113 & 116 & 120 & 123 & & & \\
\hline Veh & 12 & 12 & 12 & 12 & 12 & 12 & 12 & 12 & 11 & & & \\
\hline Ril30 & 12 & 12 & 12 & 12 & 12 & 12 & 11 & 11 & 11 & & & \\
\hline Fas10 & 11 & 11 & 11 & 11 & 11 & 11 & 11 & 11 & 11 & & & \\
\hline Fas30 & 12 & 12 & 12 & 12 & 12 & 12 & 12 & 12 & 10 & & & \\
\hline Fas10Ril30 & 12 & 12 & 12 & 12 & 12 & 12 & 11 & 10 & 10 & & & \\
\hline $\mathbf{W t}$ & 7 & 7 & 7 & 7 & 7 & 7 & 7 & 7 & 7 & & & \\
\hline
\end{tabular}

Es kann eine kontinuierliche Verschlechterung der motorischen Leistung im Rotarod ab dem Lebenstag 88 in den Gruppen Veh, Ril30, Fas10 und Fas10Ril30 beobachtet werden (Abbildung 16). Diese Verschlechterung kann bei der Fas30-Gruppe erst ab dem Lebenstag 106 in ähnlich rasantem Tempo wie bei den anderen Gruppen beobachtet werden.

In der ANOVA mit Messwiederholungen zeigen sich zwischen keiner Behandlungsgruppe (Ril30, Fas10, Fas30, Fas10Ril30) signifikante Unterschiede im LSD-Test im Vergleich zur unbehandelten Veh-Kontrollgruppe. Ein signifikanter Unterschied zeigte sich zwischen der Veh-Gruppe und der gesunden Wt-Kontrollruppe $(\mathrm{p}=0,013)$. Im Vergleich zur gesunden Wt-Kontrollgruppe sind die Unterschiede der Behandlungsgruppen Ril30 ( $\mathrm{p}=0,009)$, Fas10 $(\mathrm{p}=0,026)$, Fas10Ril30 $(\mathrm{p}=0,010)$ ebenso signifikant. Es findet sich allerdings kein signifikanter Unterschied zwischen der gesunden Wt-Kontrollgruppe und der Fas30Gruppe $(p=0,087)$. 


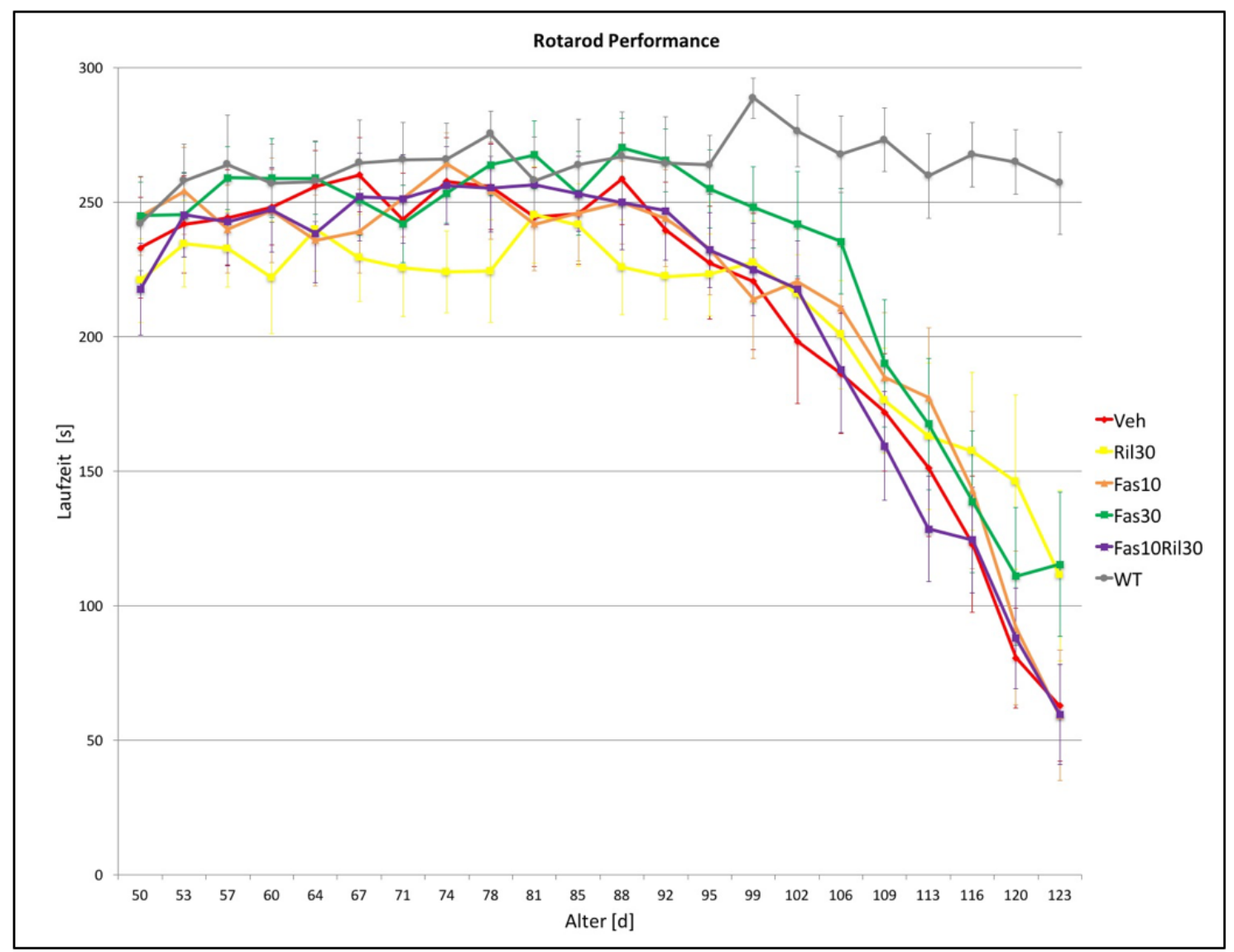

Abbildung 16. Drehwalzenversuch: Mittlere Laufzeit

Durchschnittliche Laufzeit beim Drehwalzenversuch (Rotarod) von Lebenstag 50 bis Lebenstag 123: Untersucht werden SOD1G93A-transgene weibliche Tiere, die mit Wasser (Veh), 30 mg/kg KG Riluzol (Ril30), 10 mg/kg KG Fasudil (Fas10), $30 \mathrm{mg} / \mathrm{kg} \mathrm{KG} \mathrm{Fasudil} \mathrm{(Fas30)} \mathrm{und} 10 \mathrm{mg} / \mathrm{kg}$ KG Fasudil in Kombination mit $30 \mathrm{mg} / \mathrm{kg}$ KG Riluzol (Fas10Ril30) ab dem Lebenstag 50 behandelt werden. Als gesunde Kontrollgruppe werden 7 SOD1-Wildtyp-Tiere (W'T) untersucht. Anzahl der untersuchten transgenen Tiere je Versuchstag und Gruppe: siehe Tabelle 5. Angegeben sind Mittelwerte +/- Stdf.

\subsubsection{Videobasierte Analyse des Gangs bei sich frei bewegenden Mäusen (CatWalk XT)}

Die videobasierte Analyse des Gangs dient im Rahmen der Erkrankung der SOD1G93Atransgenen Mäuse dazu, die Veränderungen im Gangbild zu beobachten und zu dokumentieren. Insbesondere die Hinterläufe sind einer frühen Beeinträchtigung unterworfen, welche auch zu dem für Stufe 2 des klinisch-neurologischen Scores charakteristischen „Watschelgang“ führt (siehe Kapitel 2.7.1.1). Die Ganganalyse unter präsymptomatischer Behandlung wird im Rahmen der Verlaufsstudie, beginnend ab Lebenstag 50, alle 7 Tage durchgeführt. Ein durchgeführter Testlauf beinhaltet drei komplette den Anforderungen entsprechende Durchläufe der Maus (siehe Kapitel 2.7.3). Dabei wird der Mittelwert der erhobenen Parameter an einem Tag gebildet und geht in die Auswertung ein. Ausgewertet wird als Hauptparameter die Durchschnittsgeschwindigkeit (mean average speed). Daneben werden die Defizite im Gebrauch der Hinterläufe mithilfe der 
folgenden Parameter des rechten Hinterlaufs analysiert: Auftrittsfläche (print area), Schrittgeschwindigkeit (swing speed), Fußlänge (print length) und Schrittlänge (stride length). Zum Vergleich werden diese Parameter auch für den rechten Vorderlauf ausgewertet.

\begin{tabular}{|l|c|c|c|c|c|c|c|c|c|c|c|c|}
\hline $\begin{array}{c}\text { Untersuchungs- } \\
\text { tag }\end{array}$ & $\mathbf{5 0}$ & $\mathbf{5 7}$ & $\mathbf{6 4}$ & $\mathbf{7 1}$ & $\mathbf{6 4}$ & $\mathbf{8 5}$ & $\mathbf{9 2}$ & $\mathbf{9 9}$ & $\mathbf{1 0 6}$ & $\mathbf{1 1 3}$ & $\mathbf{1 2 0}$ & $\mathbf{1 2 7}$ \\
\hline Veh & 12 & 12 & 12 & 12 & 12 & 12 & 12 & 10 & 12 & 11 & 9 & 9 \\
\hline Ri130 & 12 & 12 & 12 & 12 & 12 & 11 & 11 & 12 & 12 & 11 & 11 & 8 \\
\hline Fas10 & 11 & 11 & 11 & 11 & 11 & 11 & 9 & 11 & 11 & 10 & 10 & 5 \\
\hline Fas30 & 11 & 11 & 11 & 11 & 11 & 11 & 9 & 11 & 11 & 11 & 7 & 7 \\
\hline Fas10Ri130 & 12 & 12 & 12 & 12 & 12 & 12 & 11 & 11 & 12 & 12 & 9 & 7 \\
\hline Wt & 7 & 7 & 7 & 7 & 7 & 7 & 7 & 7 & 7 & 7 & 7 & 7 \\
\hline
\end{tabular}

Tabelle 6. Videobasierte Ganganalyse (CatWalk XT): Anzahl durchgeführter Testläufe.

Ein Testlauf entspricht 3 Durchläufen/Videos einer Maus.

Da die Teilnahme an der videobasierten Analyse des Gangs bei sich frei bewegenden Mäusen recht hohe motorische Anforderungen an die Tiere stellt, die die Mäuse insbesondere bei fortgeschrittener Erkrankung oft nicht mehr erfüllen, können in den letzten Lebenstagen viele erkrankte Mäuse nicht mehr am Versuch teilnehmen. Um an jedem Versuchstag jedoch mindestens 5 Testläufe je Gruppe auswerten zu können, wird als letzter ausgewerteter Versuchstag der Lebenstag 127 gewählt. An diesem Tag können noch 9 Tiere in der Veh-Gruppe, 8 Tiere der Ril30-Gruppe, 5 Tiere der Fas10-Gruppe, 7 Tiere der Fas30-Gruppe und 7 Tiere der Fas10Ril30-Gruppe am Versuch teilnehmen. Das ergibt 12 Untersuchungszeitpunkte zwischen Lebenstag 50 und Lebenstag 127. Eine Maus aus der Fas30-Gruppe wird aufgrund eines gestörten Gangbildes im Sinne eines andauernden Im-Kreise-Laufens vom Versuch ausgeschlossen, da keine Aufnahme eines den Versuchskriterien entsprechenden Durchlaufes gelang. Eine vollständige Auflistung der Anzahl aufgenommener und ausgewerteter Testläufe findet sich in Tabelle 6, wobei ein Testlauf jeweils aus 3 Durchläufen/Videos einer Maus besteht.

\subsubsection{Durchschnittsgeschwindigkeit (mean average speed)}

Die Durchschnittsgeschwindigkeit entwickelt sich bis einschließlich dem Tag 99 in allen Gruppen in etwa konstant. Es kann dann eine kontinuierliche Verlangsamung der Durchschnittsgeschwindigkeit in den Gruppen Veh, Ril30, Fas10, Fas30 undFas10Ril30 ab dem Tag 106 beobachtet werden (Abbildung 17). Die gesunden Tiere der WtKontrollgruppe bewegen sich über die gesamte Zeit dem Gedeihen entsprechend konstant schnell. 
In der ANOVA mit Messwiederholungen der 12 Untersuchungszeitpunkte ist keine Gruppe (Ril30: $p=0,850$; Fas10: $p=0,515$; Fas30: $p=0,512$; Fas10Ril30: $p=0,835$; Wt: $\mathrm{p}=0,077)$ signifikant besser als die unbehandelte Veh-Kontrollgruppe.

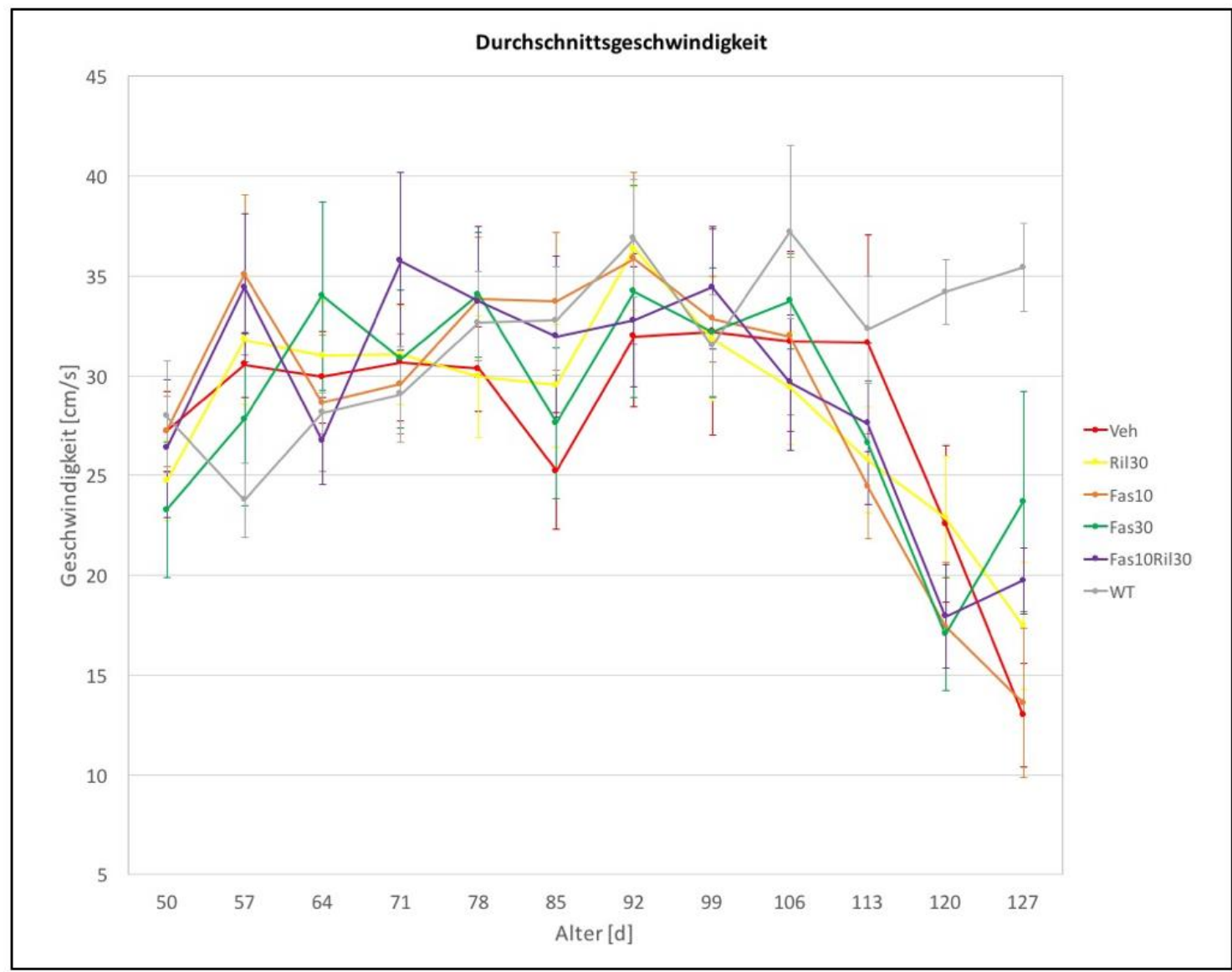

Abbildung 17. Videobasierte Ganganalyse: Durchschnittsgeschwindigkeit

Untersucht werden SOD1G93A-transgene weibliche Tiere, die mit Wasser (Veh), $30 \mathrm{mg} / \mathrm{kg}$ KG Riluzol (Ril30), 10 mg/kg KG Fasudil (Fas10), 30 mg/kg KG Fasudil (Fas30) und 10 mg/kg KG Fasudil in Kombination mit 30 mg/kg KG Riluzol (Fas10Ril30) ab dem Lebenstag 50 behandelt werden. Als gesunde Kontrollgruppe werden 7 SOD1-Wildtyp-Tiere (WT) untersucht. Anzahl der untersuchten transgenen Tiere je Versuchstag und Gruppe: siehe Tabelle 6. Angegeben sind Mittelwerte +/- Stdf.

Am letzten Untersuchungszeitpunkt, dem Tag 127, kann eine signifikant höhere Durchschnittgeschwindigkeit in der Fas30-Gruppe festgestellt werden: Sie bewegen sich am Tag 127 signifikant schneller $(p=0,021)$ als die Tiere der unbehandelten VehKontrollgruppe. Auch die gesunden Tiere der Wt-Gruppe bewegen sich signifikant schneller $(p=0,000)$ als die unbehandelte Veh-Kontrollgruppe. Die Unterschiede der anderen Gruppen (Ril30: $p=0,298$; Fas10: $p=0,896$; Fas10Ril30: $p=0,138$ ) zur VehKontrollgruppe sind nicht signifikant. Im Vergleich zur gesunden Wt-Kontrollgruppe bewegen sich alle anderen Gruppen an diesem Tag signifikant langsamer.

Am Tag 127 beträgt die Durchschnittsgeschwindigkeit in der unbehandelten VehKontrollgruppe $12,98+/-2,61 \mathrm{~cm} / \mathrm{s}$, in der Ril30-Gruppe $17,45+/-3,2 \mathrm{~cm} / \mathrm{s}$, in der 
Fas10-Gruppe 13,6 +/- 3,76, in der Fas30-Gruppe 23,69 +/-5,5 cm/s, in der Fas10Ril30Gruppe 19,71 +/- 1,7 cm/s, in der gesunden Wt-Kontrollgruppe 35,43 +/- 2,21 cm/s.

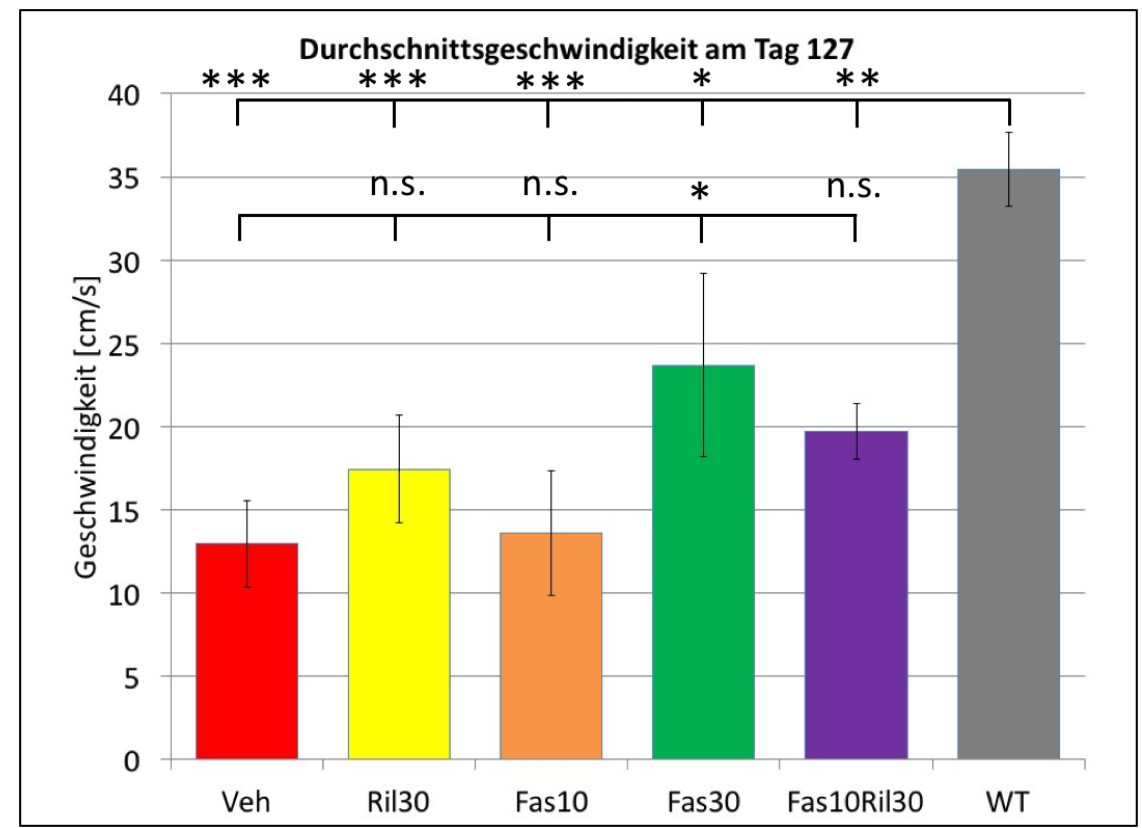

Abbildung 18. Durchschnittsgeschwindigkeit am Tag 127

Untersucht werden SOD1G93A-transgene weibliche Tiere, die mit Wasser (Veh, n=9), 30 mg/kg KG Riluzol (Ril30, n=8), $10 \mathrm{mg} / \mathrm{kg} \mathrm{KG} \mathrm{Fasudil} \mathrm{(Fas10,} \mathrm{n=5),} 30 \mathrm{mg} / \mathrm{kg}$ KG Fasudil (Fas30, n=7) und $10 \mathrm{mg} / \mathrm{kg}$ KG Fasudil in Kombination mit $30 \mathrm{mg} / \mathrm{kg}$ KG Riluzol (Fas10Ril30, n=7) ab dem Lebenstag 50 behandelt werden. Als gesunde Kontrollgruppe werden 7 SOD1-Wildtyp-Tiere (WT) untersucht. Signifikante Unterschiede zwischen den Gruppen sind bei $\mathrm{p}<0,05$ mit $\left(^{*}\right), \mathrm{p}<0,01$ mit $\left(^{* *}\right)$ und $\mathrm{p}<0,001 \mathrm{mit}\left({ }^{* *}\right)$ markiert, n. s. nicht signifikant. Angegeben sind Mittelwerte + - Stdf.

\subsubsection{Auftrittsfläche (print area) des rechten Vorderlaufs}

Es kann keine Veränderung der Auftrittsfläche des rechten Vorderlaufes im Laufe des Krankheitsprogresses in den Gruppen Ril30, Fas10, Fas30 und Fas10Ril30 und der gesunden Wt-Kontrollgruppe im Laufe des Untersuchungszeitraumes beobachtet werden. In der ANOVA mit Messwiederholungen der 12 Untersuchungszeitpunkte zeigt keine Gruppe (Ril30: $p=0,149$; Fas10: $p=0,216$; Fas30: $p=0,315$; Fas10Ril30: $p=0,145$; Wt: $\mathrm{p}=0,100)$ einen signifikanten Unterschied zur unbehandelten Kontrollgruppe. Auch im Vergleich zur gesunden Wt-Kontrollgruppe zeigen sich keine signifikanten Unterschiede (Veh: $p=0,100 ;$ Ril30: $p=0,881 ;$ Fas10: $p=0,957$; Fas30: $p=0,768$; Fas10Ril30: $p=0,963$ ).

Es fällt eine Erhöhung der Auftrittsfläche des rechten Vorderlaufes ab dem Tag 99 bei der unbehandelten Veh-Kontrollgruppe auf: Diese ist in der ANOVA mit Messwiederholungen in den fünf Untersuchungszeitpunkten (Tag 99 bis Tag 127) signifikant höher als die der gesunden Wt-Kontrollgruppe $(\mathrm{p}=0,019)$, im Gegensatz zu den behandelten Gruppen, die keinen signifikanten Unterschied zur gesunden Wt-Kontrollgruppe zeigen (Ril30: $\mathrm{p}=0,549$; Fas10: $\mathrm{p}=0,251$; Fas30: $\mathrm{p}=0,718$; Fas10Ril30: $\mathrm{p}=0,453)$. Die behandelten 
Gruppen zeigen in diesen Zeitraum jedoch keine signifikant kleinere Auftrittsfläche als die unbehandelte Veh-Kontrollgruppe (Ril30: $p=0,083$; Fas10: $p=0,344 ;$ Fas30: $p=0,088$; Fas10Ril30: $\mathrm{p}=0,139)$.

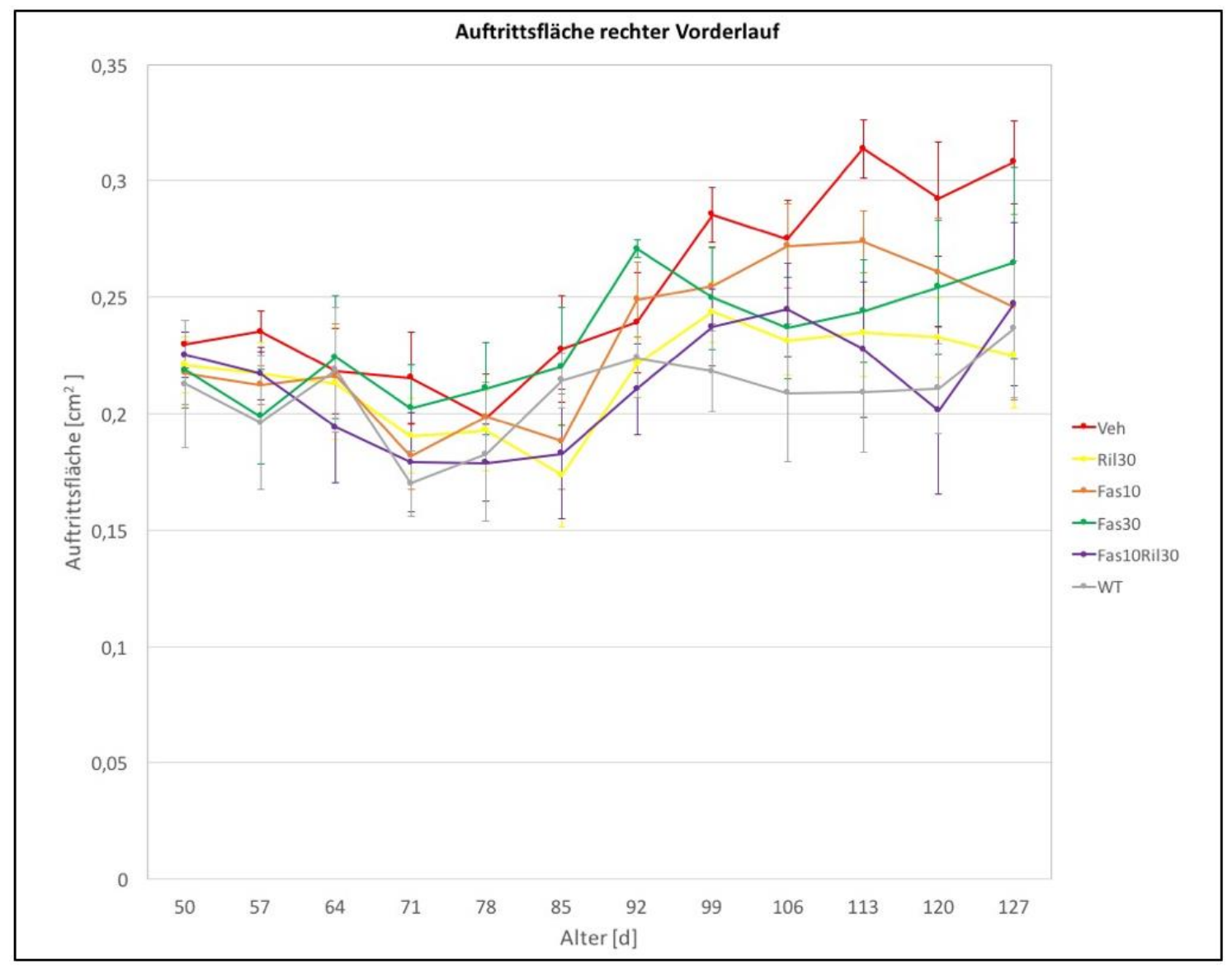

Abbildung 19. Videobasierte Ganganalyse: Auftrittsfläche rechter Vorderlauf

Untersucht werden SOD1G93A-transgene weibliche Tiere, die mit Wasser (Veh), $30 \mathrm{mg} / \mathrm{kg}$ KG Riluzol (Ril30), 10 mg/kg KG Fasudil (Fas10), 30 mg/kg KG Fasudil (Fas30) und 10 mg/kg KG Fasudil in Kombination mit 30 mg/kg KG Riluzol (Fas10Ril30) ab dem Lebenstag 50 behandelt werden. Als gesunde Kontrollgruppe werden 7 SOD1-Wildtyp-Tiere (WT) untersucht. Anzahl der untersuchten transgenen Tiere je Versuchstag und Gruppe: siehe Tabelle 6. Angegeben sind Mittelwerte +/- Stdf.

Am letzten Untersuchungszeitpunkt, dem Tag 127, kann eine Vergrößerung der Auftrittsfläche des rechten Vorderlaufs in der unbehandelten Veh-Gruppe festgestellt werden: Die Tiere haben am Tag eine signifikant größere Auftrittsfläche als die Tiere der gesunden Wt-Kontrollgruppe $(\mathrm{p}=0,036)$. Im Vergleich zur unbehandelten VehKontrollgruppe haben die Tiere der Ril30-Gruppe eine signifikant kleinere Auftrittsfläche $(p=0,044)$, die in etwa der gesunden Wt-Kontrollgruppe entspricht.

Die Unterschiede der anderen Gruppen (Fas10: p=0,150; Fas30: p=0,284; Fas10Ril30: $\mathrm{p}=0,118)$ zur Veh-Kontrollgruppe sind nicht signifikant. Im Vergleich zur gesunden WtKontrollgruppe zeigen die behandelten Gruppen keine signifikanten Unterschiede (Ril30: $\mathrm{p}=0,996 ;$ Fas10: $\mathrm{p}=0,624 ;$ Fas30: $\mathrm{p}=0,248 ;$ Fas10Ril30: $\mathrm{p}=0,585)$. 
Am Tag 127 beträgt die durchschnittliche Auftrittsfläche des rechten Vorderlaufes in der unbehandelten Veh-Kontrollgruppe 0,31 +/- 0,02 $\mathrm{cm}^{2}$, in der Ril30-Gruppe 0,22 +/- 0,02 $\mathrm{cm}^{2}$, in der Fas10-Gruppe 0,25 +/- 0,04 $\mathrm{cm}^{2}$, in der Fas30-Gruppe 0,26 +/- 0,04 $\mathrm{cm}^{2}$, in der Fas10Ril30-Gruppe 0,25 +/- 0,04 $\mathrm{cm}^{2}$, in der gesunden Wt-Kontrollgruppe 0,22 +/$0,02 \mathrm{~cm}^{2}$.

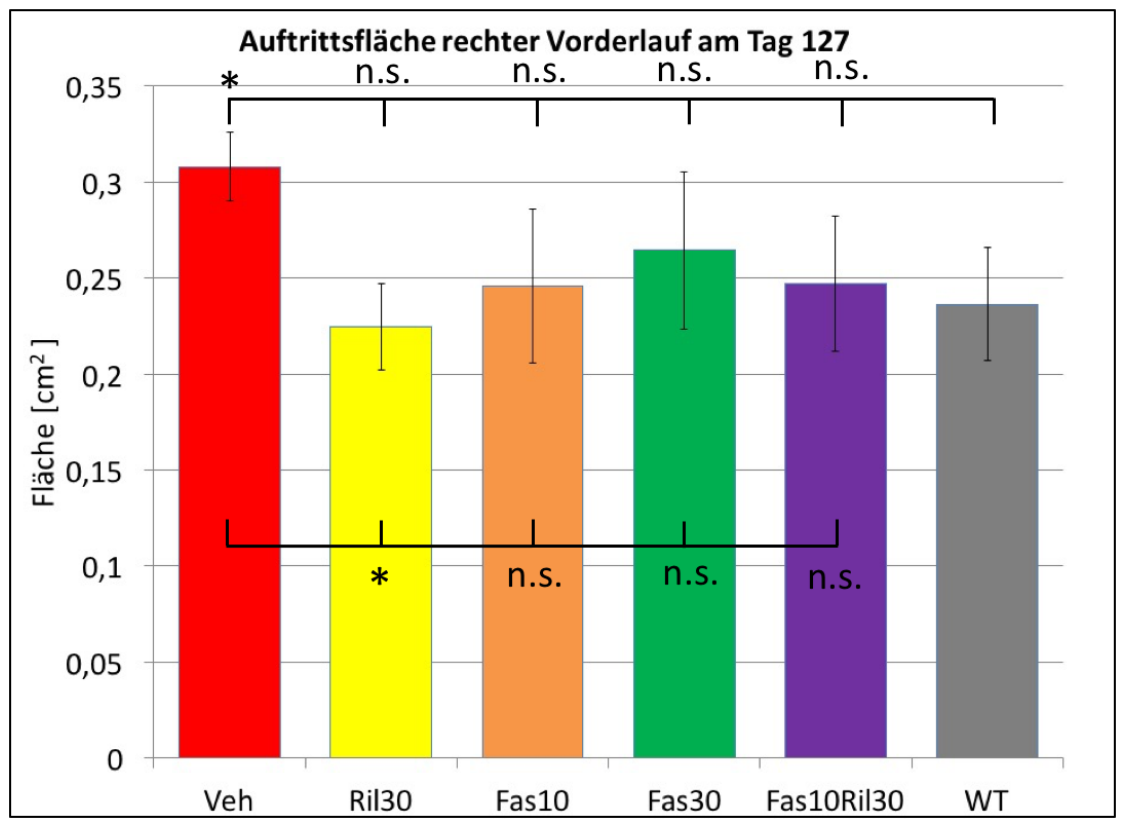

Abbildung 20. Auftrittsfläche rechter Vorderlauf am Tag 127

Untersucht werden SOD1G93A-transgene weibliche Tiere, die mit Wasser (Veh, n=9), $30 \mathrm{mg} / \mathrm{kg} \mathrm{KG} \mathrm{Riluzol}$ (Ril30, n=8), $10 \mathrm{mg} / \mathrm{kg} \mathrm{KG}$ Fasudil (Fas10, n=5), $30 \mathrm{mg} / \mathrm{kg} \mathrm{KG} \mathrm{Fasudil} \mathrm{(Fas30,} \mathrm{n=7)} \mathrm{und} 10 \mathrm{mg} / \mathrm{kg} \mathrm{KG}$ Fasudil in Kombination mit $30 \mathrm{mg} / \mathrm{kg}$ KG Riluzol (Fas10Ril30, n=7) ab dem Lebenstag 50 behandelt werden. Als gesunde Kontrollgruppe werden 7 SOD1-Wildtyp-Tiere (WT) untersucht. Signifikante Unterschiede zwischen den Gruppen sind bei $\mathrm{p}<0,05$ mit $\left(^{*}\right)$ markiert, n. s. nicht signifikant. Angegeben sind Mittelwerte +/- Stdf.

\subsubsection{Auftrittsfläche (print area) des rechten Hinterlaufs}

Die Auftrittsfläche des rechten Hinterlaufs entwickelt sich bis einschließlich dem Tag 99 in allen Gruppe in etwa konstant. Es kann dann eine kontinuierliche Verkleinerung der Auftrittsfläche des rechten Hinterlaufs im Laufe des Krankheitsprogresses in den Gruppen Veh, Ril30, Fas30 und Fas10Ril30 ab dem Tag 106 beobachtet werden. Ein Abfall der Auftrittsfläche in der Fas10-Gruppe ist erst am Tag $127 \mathrm{zu}$ beobachten. Die gesunden Tiere der Wt-Kontrollgruppe haben über die gesamte Zeit dem Gedeihen entsprechend eine konstante Auftrittsfläche.

In der ANOVA mit Messwiederholungen der 12 Untersuchungszeitpunkte ist keine Gruppe (Ril30: $p=0,165$; Fas10: $p=0,495$; Fas30: $p=0,549$; Fas10Ril30: $p=0,451$; Wt: $\mathrm{p}=0,590)$ signifikant besser als die unbehandelte Kontrollgruppe. Im Vergleich zur gesunden Wt-Kontrollgruppe ist keine Gruppe signifikant schlechter (Veh: p=0,590 Ril30: $\mathrm{p}=0,386 ;$ Fas10: $\mathrm{p}=0,275 ;$ Fas30: $\mathrm{p}=0,855 ;$ Fas10Ril30: $\mathrm{p}=0,766)$. 


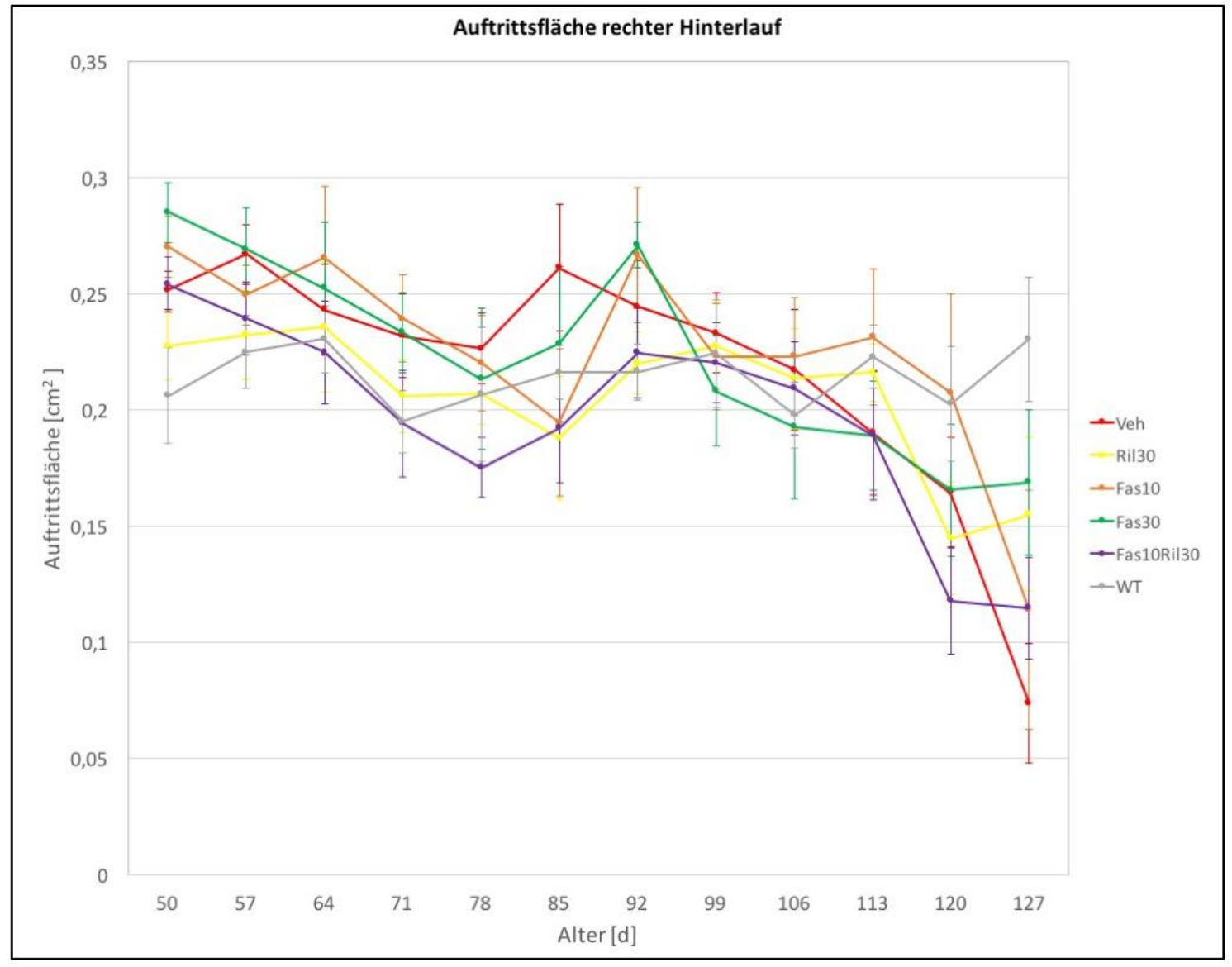

Abbildung 21. Videobasierte Ganganalyse: Auftrittsfläche rechter Hinterlauf

Untersucht werden SOD1G93A-transgene weibliche Tiere, die mit Wasser (Veh), $30 \mathrm{mg} / \mathrm{kg}$ KG Riluzol (Ril30), 10 mg/kg KG Fasudil (Fas10), 30 mg/kg KG Fasudil (Fas30) und 10 mg/kg KG Fasudil in Kombination mit 30 mg/kg KG Riluzol (Fas10Ril30) ab dem Lebenstag 50 behandelt werden. Als gesunde Kontrollgruppe werden 7 SOD1-Wildtyp-Tiere (WT) untersucht. Anzahl der untersuchten transgenen Tiere je Versuchstag und Gruppe: siehe Tabelle 6. Angegeben sind Mittelwerte +/- Stdf.

Am letzten Untersuchungszeitpunkt, dem Tag 127, kann eine Verbesserung der Tiere der Fas30-Gruppe festgestellt werden: Sie haben am Tag 127 eine signifikant größere Auftrittsfläche $(\mathrm{p}=0,031)$ als die Tiere der unbehandelten Veh-Kontrollgruppe. Auch die gesunden Tiere der Wt-Gruppe haben eine signifikant größere Auftrittsfläche $(p=0,000)$ als die unbehandelte Veh-Kontrollgruppe. Die Unterschiede der anderen Gruppen (Ril30: $\mathrm{p}=0,052$; Fas10: $\mathrm{p}=0,377$; Fas10Ril30: $\mathrm{p}=0,318$ ) zur Veh-Kontrollgruppe sind nicht signifikant. Im Vergleich zur gesunden Wt-Kontrollgruppe haben neben der unbehandelten Veh-Kontrollgruppe auch die Fas10-Gruppe $(\mathrm{p}=0,018)$ und die Fas10Ril30-Gruppe $(\mathrm{p}=0,011)$ eine signifikant kleinere Auftrittsfläche, nicht jedoch die Fas30-Gruppe $(\mathrm{p}=0,177)$ und die Ril30-Gruppe $(\mathrm{p}=0,088)$.

Am Tag 127 beträgt die durchschnittliche Auftrittsfläche des rechten Hinterlaufes in der unbehandelten Veh-Kontrollgruppe 0,07+/- 0,03 $\mathrm{cm}^{2}$, in der Ril30-Gruppe 0,15 +/- 0,03 $\mathrm{cm}^{2}$, in der Fas10-Gruppe 0,11+/- 0,05 $\mathrm{cm}^{2}$, in der Fas30-Gruppe 0,17+/- 0,03 $\mathrm{cm}^{2}$, in 
der Fas10Ril30-Gruppe 0,11 +/- 0,02 $\mathrm{cm}^{2}$, in der gesunden Wt-Kontrollgruppe 0,23 +/$0,03 \mathrm{~cm}^{2}$.

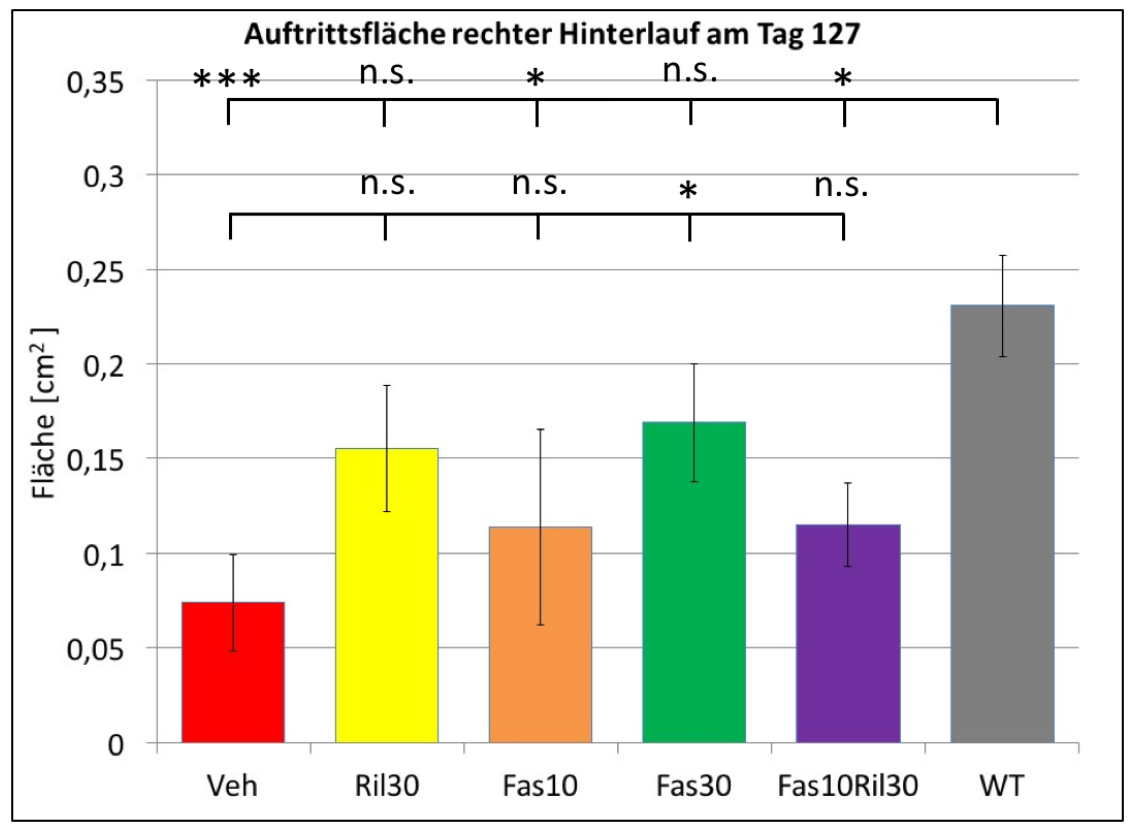

Abbildung 22. Auftrittsfläche rechter Hinterlauf am Tag 127

Untersucht werden SOD1G93A-transgene weibliche Tiere, die mit Wasser (Veh, $\mathrm{n}=9$ ), $30 \mathrm{mg} / \mathrm{kg} \mathrm{KG}$ Riluzol (Ril30, $\mathrm{n}=8$ ), $10 \mathrm{mg} / \mathrm{kg} \mathrm{KG}$ Fasudil (Fas10, n=5), $30 \mathrm{mg} / \mathrm{kg} \mathrm{KG} \mathrm{Fasudil} \mathrm{(Fas30,} \mathrm{n=7)} \mathrm{und} 10 \mathrm{mg} / \mathrm{kg} \mathrm{KG}$ Fasudil in Kombination mit $30 \mathrm{mg} / \mathrm{kg}$ KG Riluzol (Fas10Ril30, n=7) ab dem Lebenstag 50 behandelt werden. Als gesunde Kontrollgruppe werden 7 SOD1-Wildtyp-Tiere (WT) untersucht. Signifikante Unterschiede zwischen den Gruppen sind bei $\mathrm{p}<0,05$ mit $\left(^{*}\right)$ und $\mathrm{p}<0,001$ mit $(* * *)$ markiert, n. s. nicht signifikant. Angegeben sind Mittelwerte +/- Stdf.

\subsubsection{Auftrittslänge (print length) des rechten Vorderlaufs}

Es kann keine starke Veränderung der Auftrittslänge des rechten Vorderlaufes im Laufe des Krankheitsprogresses in den Gruppen Veh, Ril30, Fas10, Fas30 und Fas10Ril30 und der gesunden Wt-Kontrollgruppe im Laufe des Untersuchungszeitraumes beobachtet werden.

In der ANOVA mit Messwiederholungen der 12 Untersuchungszeitpunkte zeigt keine Gruppe (Ril30: $p=0,149$; Fas10: $p=0,373$; Fas30: $p=0,445$; Fas10Ril30: $p=0,417$; Wt: $\mathrm{p}=0,474)$ einen signifikanten Unterschied zur unbehandelten Veh-Kontrollgruppe. Auch im Vergleich zur gesunden Wt-Kontrollgruppe zeigen sich keine signifikanten Unterschiede (Ril30: $\mathrm{p}=0,454$; Fas10: $\mathrm{p}=0,734$; Fas30: $\mathrm{p}=0,878$; Fas10Ril30: $\mathrm{p}=0,839$ ).

Auch am letzten Untersuchungstag, Tag 127, zeigen sich keine signifikanten Unterschiede zwischen den Gruppen. 


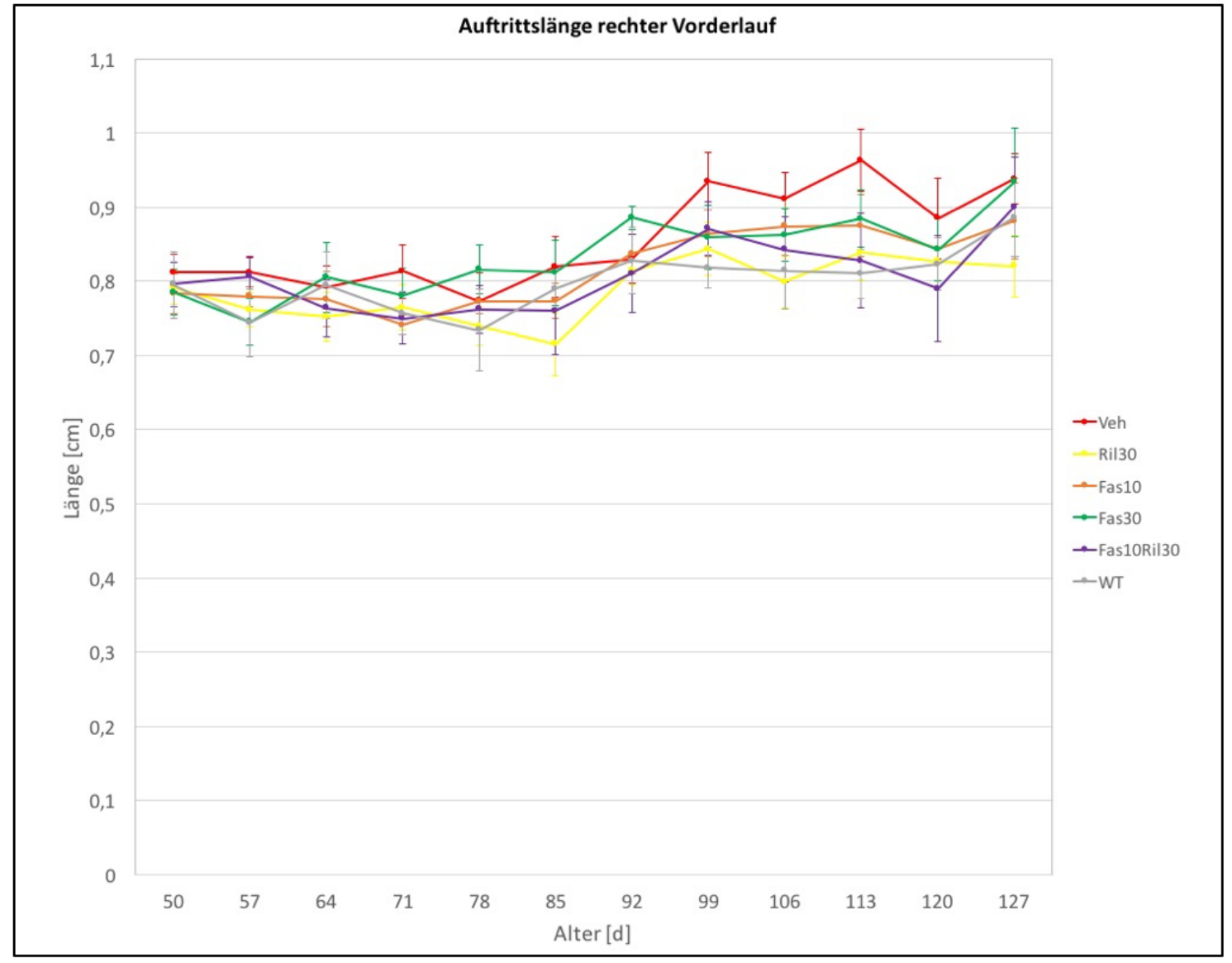

Abbildung 23. Videobasierte Ganganalyse: Auftrittslänge rechter Vorderlauf

Untersucht werden SOD1G93A-transgene weibliche Tiere, die mit Wasser (Veh), $30 \mathrm{mg} / \mathrm{kg}$ KG Riluzol (Ril30), 10 mg/kg KG Fasudil (Fas10), 30 mg/kg KG Fasudil (Fas30) und 10 mg/kg KG Fasudil in Kombination mit 30 mg/kg KG Riluzol (Fas10Ril30) ab dem Lebenstag 50 behandelt werden. Als gesunde Kontrollgruppe werden 7 SOD1-Wildtyp-Tiere (WT) untersucht. Anzahl der untersuchten transgenen Tiere je Versuchstag und Gruppe: siehe Tabelle 6. Angegeben sind Mittelwerte +/- Stdf.

\subsubsection{Auftrittslänge (print length) des rechten Hinterlaufs}

Es kann eine kontinuierliche Verkürzung der Auftrittslänge des rechten Hinterlaufes im Laufe des Krankheitsprogresses in der unbehandelten Veh-Kontrollgruppe, in der Ril30Gruppe, in der Fas10-Gruppe und der Fas10Ril30-Gruppe ab dem Tag 106 beobachtet werden. Die gesunden Tiere der Wt-Kontrollgruppe und die Tiere der Fas30-Gruppe haben über die gesamte Zeit eine konstante Auftrittslänge.

In der ANOVA mit Messwiederholungen der 12 Untersuchungszeitpunkte zeigen sich keine signifikanten Unterschiede zur unbehandelten Veh-Kontrollgruppe (Ril30: $\mathrm{p}=0,851$; Fas10: $\mathrm{p}=0,564$; Fas30: $\mathrm{p}=0,094$; Fas10Ril30: $\mathrm{p}=0722$; Wt: $\mathrm{p}=0,430$ ). Im Vergleich zur gesunden Wt-Kontrollgruppe zeigen sich ebenso keine signifikanten Unterschiede (Ril30: $\mathrm{p}=0,580 ;$ Fas10: $\mathrm{p}=0,979 ;$ Fas30: $\mathrm{p}=0,334$; Fas10Ril30: $\mathrm{p}=0,723$ ).

Es fällt eine Verkürzung der Auftrittslänge des rechten Hinterlaufes ab dem Tag 99 bei der unbehandelten Veh-Kontrollgruppe: Die Auftrittslänge der unbehandelten Veh- 
Kontrollgruppe ist in der ANOVA mit Messwiederholungen in den fünf Untersuchungszeitpunkten (Tag 99 bis Tag127) signifikant kürzer als die der gesunden WtKontrollgruppe $(p=0,030)$. Signifikant länger als die der unbehandelten VehKontrollgruppe ist die der Fas30-Gruppe in diesem Zeitraum ( $\mathrm{p}=0,005)$. Die Unterschiede der anderen Behandlungsgruppen sind in diesen Zeitraum im Vergleich zur unbehandelten Veh-Kontrollgruppe nicht signifikant (Ril30: $p=0,075$; Fas10: $p=0,464$; Fas10Ril30: $\mathrm{p}=0$,138). Die Behandlungsgruppen zeigen allesamt keinen signifikanten Unterschied zur gesunden Wt-Gruppe (Ril30: $p=0,744$; Fas10: $p=0,335$; Fas30: $p=0,423$; Fas10Ril30: $\mathrm{p}=0,601)$.

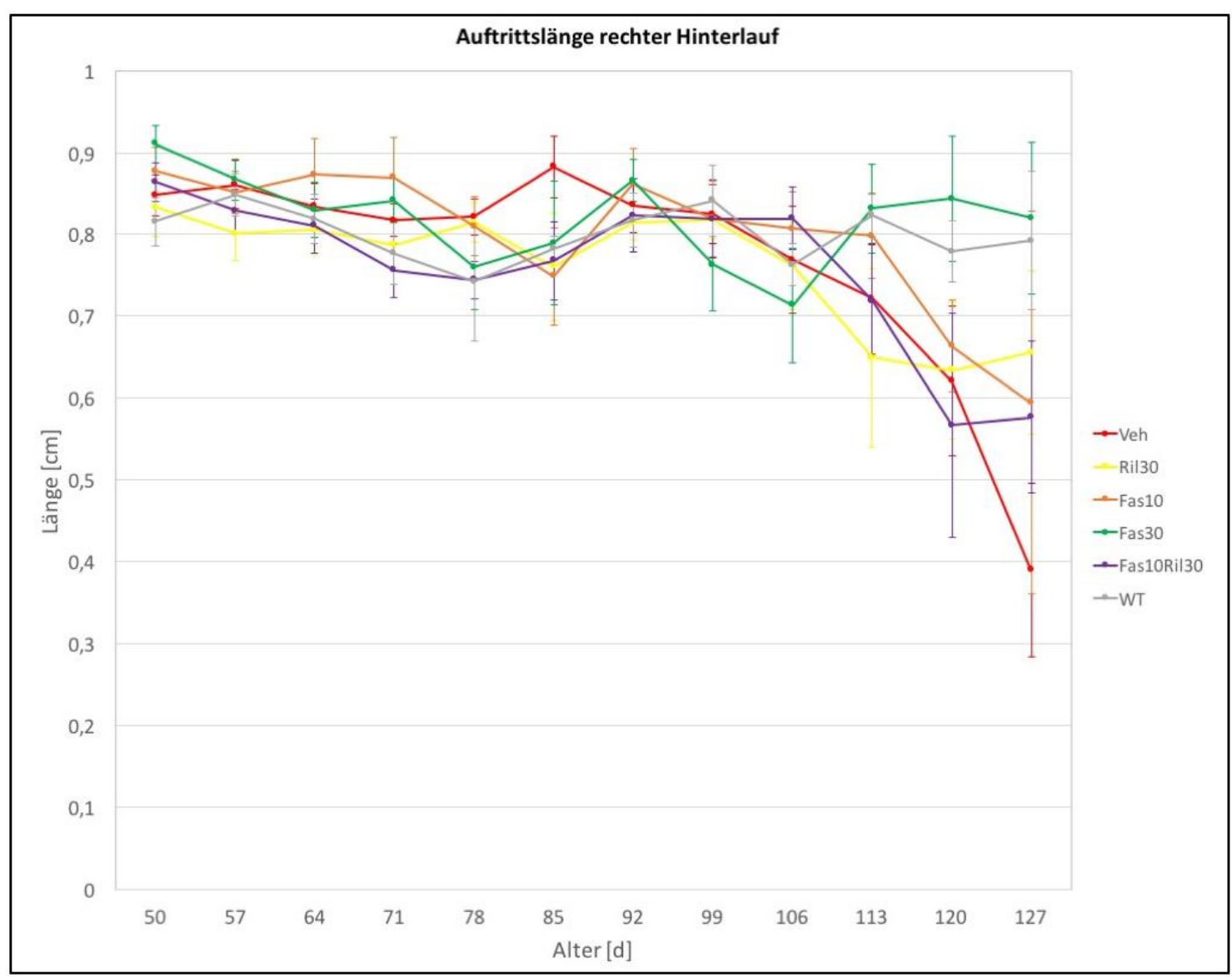

Abbildung 24. Videobasierte Ganganalyse: Auftrittslänge rechter Hinterlauf

Untersucht werden SOD1G93A-transgene weibliche Tiere, die mit Wasser (Veh), $30 \mathrm{mg} / \mathrm{kg}$ KG Riluzol (Ril30), 10 mg/kg KG Fasudil (Fas10), 30 mg/kg KG Fasudil (Fas30) und 10 mg/kg KG Fasudil in Kombination mit 30 mg/kg KG Riluzol (Fas10Ril30) ab dem Lebenstag 50 behandelt werden. Als gesunde Kontrollgruppe werden 7 SOD1-Wildtyp-Tiere (WT) untersucht. Anzahl der untersuchten transgenen Tiere je Versuchstag und Gruppe: siehe Tabelle 6. Angegeben sind Mittelwerte +/- Stdf.

Am letzten Untersuchungszeitpunkt, dem Tag 127, kann eine Verkürzung der Auftrittslänge des rechten Hinterlaufs in der unbehandelten Veh-Gruppe festgestellt werden: Die Tiere haben am Tag eine signifikant kürzere Auftrittslänge als die Tiere der gesunden Wt-Kontrollgruppe $(p=0,009)$. Im Vergleich zur unbehandelten Veh- 
Kontrollgruppe haben die Tiere der Fas30-Gruppe einen signifikant längeren Auftritt $(p=0,008)$, die in etwa der der gesunden Wt-Kontrollgruppe entspricht.

Die Unterschiede der anderen Gruppen (Ril30: $p=0,061$; Fas10: $p=0,147$; Fas10Ril30: $\mathrm{p}=0$,201) zur Veh-Kontrollgruppe sind nicht signifikant. Im Vergleich zur gesunden WtKontrollgruppe zeigen die behandelten Gruppen keine signifikanten Unterschiede (Ril30: $\mathrm{p}=0,424 ;$ Fas10: $\mathrm{p}=0,268 ;$ Fas30: $\mathrm{p}=0,877 ;$ Fas10Ril30: $\mathrm{p}=0,254)$.

Am Tag 127 beträgt die durchschnittliche Auftrittslänge des rechten Hinterlaufes in der unbehandelten Veh-Kontrollgruppe 0,35 +/- 0,11 cm, in der Ril30-Gruppe 0,66 +/- 0,1 $\mathrm{cm}$, in der Fas10-Gruppe 0,59 +/- 0,23 cm, in der Fas30-Gruppe 0,82 +/- 0,09 cm, in der Fas10Ril30-Gruppe 0,58 +/- 0,09 cm, in der gesunden Wt-Kontrollgruppe 0,79 +/- 0,08 $\mathrm{cm}$.

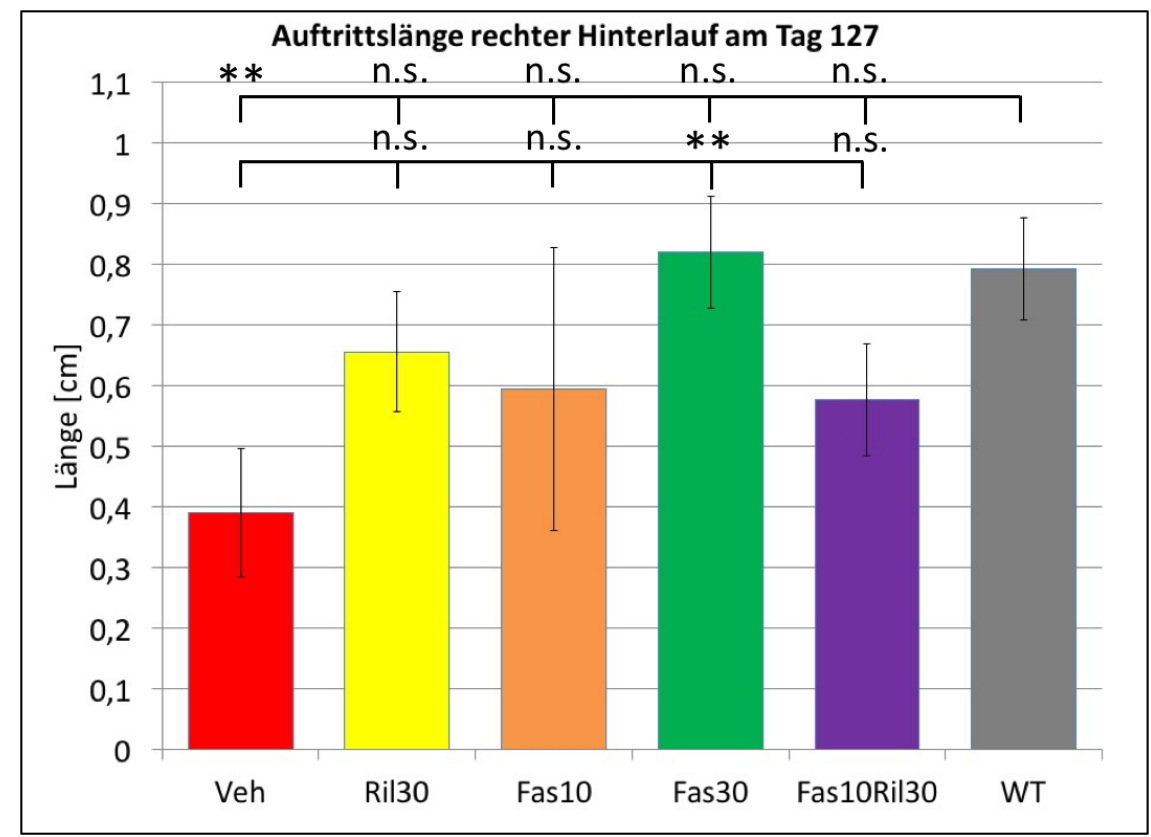

Abbildung 25. Auftrittslänge rechter Hinterlauf am Tag 127

Untersucht werden SOD1G93A-transgene weibliche Tiere, die mit Wasser (Veh, n=9), 30 mg/kg KG Riluzol (Ril30, n=8), $10 \mathrm{mg} / \mathrm{kg} \mathrm{KG} \mathrm{Fasudil} \mathrm{(Fas10,} \mathrm{n=5),} 30 \mathrm{mg} / \mathrm{kg}$ KG Fasudil (Fas30, n=7) und $10 \mathrm{mg} / \mathrm{kg} \mathrm{KG} \mathrm{Fasudil} \mathrm{in}$ Kombination mit 30 mg/kg KG Riluzol (Fas10Ril30, n=7) ab dem Lebenstag 50 behandelt werden. Als gesunde Kontrollgruppe werden 7 SOD1-Wildtyp-Tiere (WT) untersucht. Signifikante Unterschiede zwischen den Gruppen sind bei $\mathrm{p}<0,01$ mit $\left(^{* *}\right)$ markiert, n. s. nicht signifikant. Angegeben sind Mittelwerte +/- Stdf.

\subsubsection{Schrittgeschwindigkeit (swing speed) des rechten Vorderlaufs}

Die Schrittgeschwindigkeit des rechten Vorderlaufs entwickelt sich in allen Gruppen in etwa konstant bis einschließlich zum Tag 99. Ab diesem Zeitpunkt kann eine kontinuierliche Verlangsamung in der Schrittgeschwindigkeit des rechten Vorderlaufs in der unbehandelten Veh-Kontrollgruppe und den behandelten Gruppen im Gegensatz zur gesunden Wt-Kontrollgruppe beobachtet werden. Die gesunden Tiere der Wt- 
Kontrollgruppe haben über die gesamte Zeit eine konstante dem Gedeihen entsprechende Schrittgeschwindigkeit.

In der ANOVA mit Messwiederholungen aller 12 Untersuchungszeitpunkte zeigen sich keine signifikanten Unterschiede zur unbehandelten Veh-Kontrollgruppe (Ril30: $p=0,641$; Fas10: $\mathrm{p}=0,772$; Fas30: $\mathrm{p}=0,303$; Fas10Ril30: $\mathrm{p}=0,894$; Wt: $\mathrm{p}=0,193)$. Im Vergleich zur gesunden Wt-Kontrollgruppe zeigt die Fas30-Gruppe in diesem Zeitraum eine signifikant langsamere Schrittgeschwindigkeit des rechten Vorderlaufs $(\mathrm{p}=0,038)$, ansonsten zeigen sich ebenso keine signifikanten Unterschiede zur gesunden Wt-Kontrollgruppe (Ril30: $\mathrm{p}=0,082 ;$ Fas10: $\mathrm{p}=0,164$; Fas10Ril30: $\mathrm{p}=0,325)$.

Es fällt eine Verlangsamung der Schrittgeschwindigkeit des rechten Vorderlaufes ab dem Tag 106 bei der unbehandelten Veh-Kontrollgruppe und den Behandlungsgruppen auf: Die Schrittgeschwindigkeit der unbehandelten Veh-Kontrollgruppe ist in der ANOVA mit Messwiederholungen in den vier Untersuchungszeitpunkten (Tag 106 bis Tag 127) signifikant langsamer als die der gesunden Wt-Kontrollgruppe $(\mathrm{p}=0,035)$. Die Unterschiede der Behandlungsgruppen sind in diesen Zeitraum im Vergleich zur gesunden WtKontrollgruppe ebenso allesamt signifikant (Ril30: $p=0,044$; Fas10: $p=0,030$; Fas30: $\mathrm{p}=0,038$; Fas10Ril30: $\mathrm{p}=0,015)$. Die Behandlungsgruppen zeigen allesamt keinen signifikanten Unterschied zur unbehandelten Veh-Gruppe (Ril30: p=0,857; Fas10: $\mathrm{p}=0,795 ;$ Fas30: $\mathrm{p}=0,884 ;$ Fas10Ril30: $\mathrm{p}=0,654)$. 


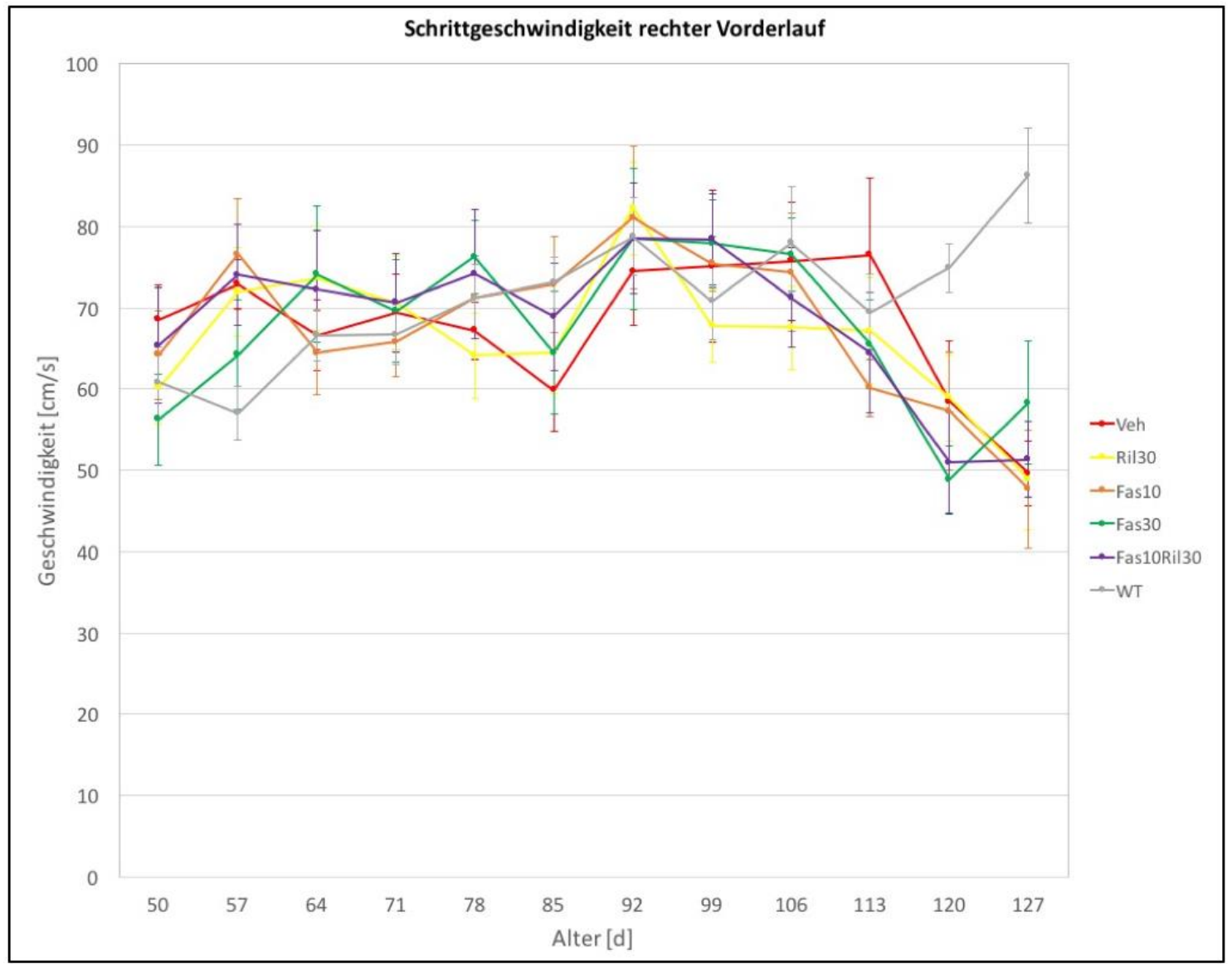

Abbildung 26. Videobasierte Ganganalyse: Schrittgeschwindigkeit rechter Vorderlauf

Untersucht werden SOD1G93A-transgene weibliche Tiere, die mit Wasser (Veh), $30 \mathrm{mg} / \mathrm{kg}$ KG Riluzol (Ril30), 10 $\mathrm{mg} / \mathrm{kg} \mathrm{KG}$ Fasudil (Fas10), $30 \mathrm{mg} / \mathrm{kg}$ KG Fasudil (Fas30) und $10 \mathrm{mg} / \mathrm{kg} \mathrm{KG}$ Fasudil in Kombination mit $30 \mathrm{mg} / \mathrm{kg} \mathrm{KG}$ Riluzol (Fas10Ril30) ab dem Lebenstag 50 behandelt werden. Als gesunde Kontrollgruppe werden 7 SOD1-Wildtyp-Tiere (WT) untersucht. Anzahl der untersuchten transgenen Tiere je Versuchstag und Gruppe: siehe Tabelle 6. Angegeben sind Mittelwerte +/- Stdf.

Am letzten Untersuchungszeitpunkt, dem Tag 127, kann eine Verlangsamung der Schrittgeschwindigkeit des rechten Vorderlaufs in der unbehandelten Veh-Gruppe und allen Behandlungsgruppen festgestellt werden: Die Tiere haben am Tag eine signifikant langsamere Schrittgeschwindigkeit als die Tiere der gesunden Wt-Kontrollgruppe $(p=0,000)$.

Die Unterschiede der Behandlungsgruppen (Ril30: p=0,914; Fas10: p=0,813; Fas30: $\mathrm{p}=0,262$; Fas10Ril30: $\mathrm{p}=0,814)$ zur Veh-Kontrollgruppe sind nicht signifikant.

Im Vergleich zur gesunden Wt-Kontrollgruppe zeigen die behandelten Gruppen allesamt eine signifikant langsamere Schrittgeschwindigkeit (Ril30: $p=0,000 ;$ Fas10: $p=0,000 ;$ Fas30: $\mathrm{p}=0,000$; Fas10Ril30: $\mathrm{p}=0,000)$.

Am Tag 127 beträgt die durchschnittliche Schrittgeschwindigkeit des rechten Vorderlaufes in der unbehandelten Veh-Kontrollgruppe 49,63 +/- 4,02 cm/s, in der Ril30-Gruppe 48,84 +/- 6,17 cm/s, in der Fas10-Gruppe 47,72 +/- 7,23 cm/s, in der Fas30-Gruppe 58,31 +/- 
$7,58 \mathrm{~cm} / \mathrm{s}$, in der Fas10Ril30-Gruppe 51,37 +/- 4,68 cm/s, in der gesunden WtKontrollgruppe $86,32+/-5,84 \mathrm{~cm} / \mathrm{s}$.

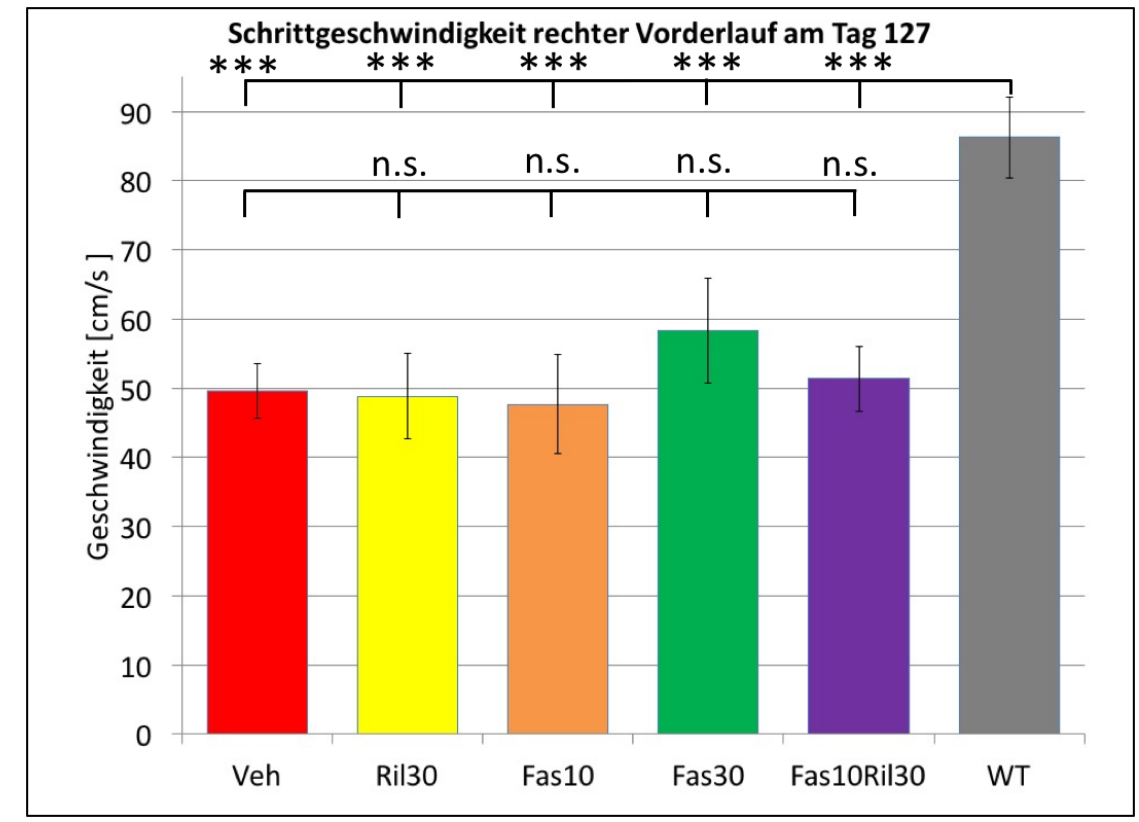

Abbildung 27. Schrittgeschwindigkeit rechter Vorderlauf am Tag 127

Untersucht werden SOD1G93A-transgene weibliche Tiere, die mit Wasser (Veh, n=9), 30 mg/kg KG Riluzol (Ril30, n=8), $10 \mathrm{mg} / \mathrm{kg} \mathrm{KG} \mathrm{Fasudil} \mathrm{(Fas10,} \mathrm{n=5),} 30 \mathrm{mg} / \mathrm{kg} \mathrm{KG} \mathrm{Fasudil} \mathrm{(Fas30,} \mathrm{n=7)} \mathrm{und} 10 \mathrm{mg} / \mathrm{kg} \mathrm{KG}$ Fasudil in Kombination mit $30 \mathrm{mg} / \mathrm{kg}$ KG Riluzol (Fas10Ril30, n=7) ab dem Lebenstag 50 behandelt werden. Als gesunde Kontrollgruppe werden 7 SOD1-Wildtyp-Tiere (WT) untersucht. Signifikante Unterschiede zwischen den Gruppen sind bei $\mathrm{p}<0,001$ mit $\left({ }^{* *}\right)$ markiert, n. s. nicht signifikant. Angegeben sind Mittelwerte +/- Stdf.

\subsubsection{Schrittgeschwindigkeit (swing speed) des rechten Hinterlaufs}

Es kann eine kontinuierliche Verlangsamung der Schrittgeschwindigkeit des rechten Hinterlaufes im Laufe des Krankheitsprogresses in der unbehandelten Veh-Kontrollgruppe und den behandelten Gruppen im Gegensatz zur gesunden Wt-Kontrollgruppe besonders ab dem Tag 99 beobachtet werden. Die gesunden Tiere der Wt-Kontrollgruppe haben über die gesamte Zeit eine konstante, dem Gedeihen entsprechende Schrittgeschwindigkeit.

In der ANOVA mit Messwiederholungen aller 12 Untersuchungszeitpunkte zeigen sich keine signifikanten Unterschiede zur unbehandelten Veh-Kontrollgruppe (Ril30: $\mathrm{p}=0,795$; Fas10: $\mathrm{p}=0,758$; Fas30: $\mathrm{p}=0,314$; Fas10Ril30: $\mathrm{p}=0,964$; Wt: $\mathrm{p}=0,237)$. Im Vergleich zur gesunden Wt-Kontrollgruppe zeigt die Fas30-Gruppe in diesem Zeitraum eine signifikant langsamere Schrittgeschwindigkeit $(p=0,050)$, ansonsten zeigen sich ebenso keine signifikanten Unterschiede zur gesunden Wt-Kontrollgruppe (Ril30: p=0,375; Fas10: $\mathrm{p}=0,536$; Fas10Ril30: $\mathrm{p}=0,333)$.

Es fällt eine Verlangsamung der Schrittgeschwindigkeit des rechten Hinterlaufes ab dem Tag 99 bei der unbehandelten Veh-Kontrollgruppe auf: Die Schrittgeschwindigkeit der unbehandelten Veh-Kontrollgruppe ist in der ANOVA mit Messwiederholungen in den 
fünf Untersuchungszeitpunkten (Tag 99 bis Tag127) signifikant langsamer als die der gesunden Wt-Kontrollgruppe $(\mathrm{p}=0,018)$. Die Unterschiede der Behandlungsgruppen sind in diesen Zeitraum im Vergleich zur unbehandelten Veh-Kontrollgruppe nicht signifikant (Ril30: $\mathrm{p}=0,266$; Fas10: $\mathrm{p}=0,424 ;$ Fas30: $\mathrm{p}=0,590$ Fas10Ril30: $\mathrm{p}=0,828$ ). Neben der unbehandelten Veh-Kontrollgruppe zeigt auch die Fas10Ril30-Gruppe eine signifikant langsamere Schrittgeschwindigkeit als die gesunde Wt-Kontrollgruppe $(p=0,047)$. Die anderen Behandlungsgruppen zeigen allesamt keinen signifikanten Unterschied zur gesunden Wt-Gruppe (Ril30: $\mathrm{p}=0,211$; Fas10: $\mathrm{p}=0,193$; Fas30: $\mathrm{p}=0,091$ ).

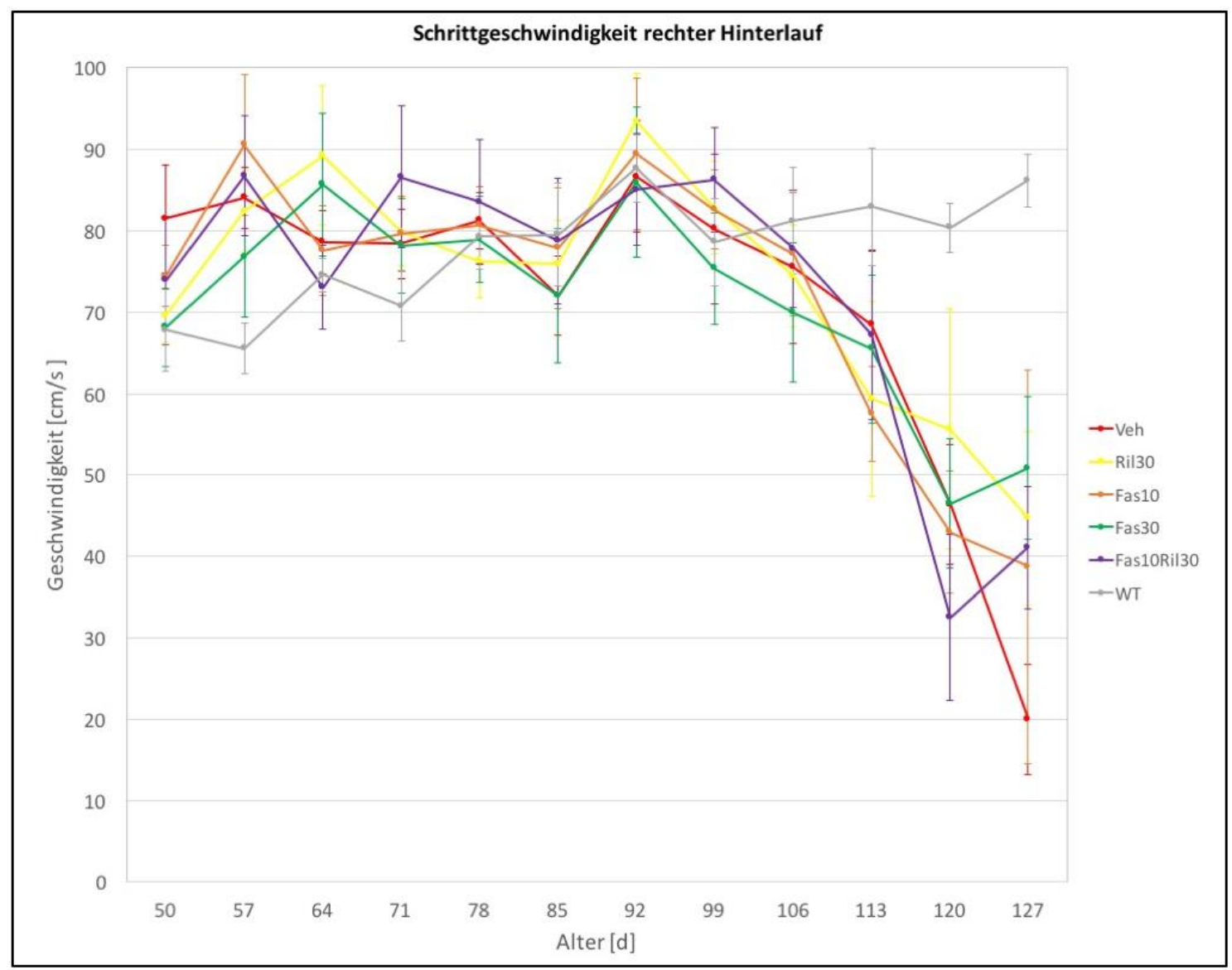

Abbildung 28. Videobasierte Ganganalyse: Schrittgeschwindigkeit rechter Hinterlauf

Untersucht werden SOD1G93A-transgene weibliche Tiere, die mit Wasser (Veh), $30 \mathrm{mg} / \mathrm{kg}$ KG Riluzol (Ril30), 10 mg/kg KG Fasudil (Fas10), 30 mg/kg KG Fasudil (Fas30) und 10 mg/kg KG Fasudil in Kombination mit 30 mg/kg KG Riluzol (Fas10Ril30) ab dem Lebenstag 50 behandelt werden. Als gesunde Kontrollgruppe werden 7 SOD1-Wildtyp-Tiere (WT) untersucht. Anzahl der untersuchten transgenen Tiere je Versuchstag und Gruppe: siehe Tabelle 6. Angegeben sind Mittelwerte +/- Stdf.

Am letzten Untersuchungszeitpunkt, dem Tag 127, kann eine Verlangsamung der Schrittgeschwindigkeit des rechten Hinterlaufs in der unbehandelten Veh-Gruppe festgestellt werden: Die Tiere haben am Tag eine signifikant langsamere Schrittgeschwindigkeit als die Tiere der gesunden Wt-Kontrollgruppe $(p=0,000)$. Im 
Vergleich zur unbehandelten Veh-Kontrollgruppe haben die Tiere der Fas30-Gruppe eine signifikant schnellere Schrittgeschwindigkeit $(\mathrm{p}=0,027)$.

Die Unterschiede der anderen Gruppen (Ril30: $p=0,073$; Fas10: $p=0,213$; Fas10Ril30: $\mathrm{p}=0,124)$ zur Veh-Kontrollgruppe sind nicht signifikant. Im Vergleich zur gesunden WtKontrollgruppe zeigen die behandelten Gruppen allesamt eine signifikant langsamere Schrittgeschwindigkeit (Ril30: $p=0,006$; Fas10: $p=0,004$; Fas30: $p=0,014$; Fas10Ril30: $\mathrm{p}=0,003)$.

Am Tag 127 beträgt die durchschnittliche Schrittgeschwindigkeit des rechten Hinterlaufes in der unbehandelten Veh-Kontrollgruppe $20+/-6,8 \mathrm{~cm} / \mathrm{s}$, in der Ril30-Gruppe 44,73 +/- 10,7 cm/s, in der Fas10-Gruppe 38,76 +/- 24,19 cm/s, in der Fas30-Gruppe 50,8 +/8,78 cm/s, in der Fas10Ril30-Gruppe 41,08 +/- 7,5 cm/s, in der gesunden WtKontrollgruppe $86,21+/-8,46 \mathrm{~cm} / \mathrm{s}$.

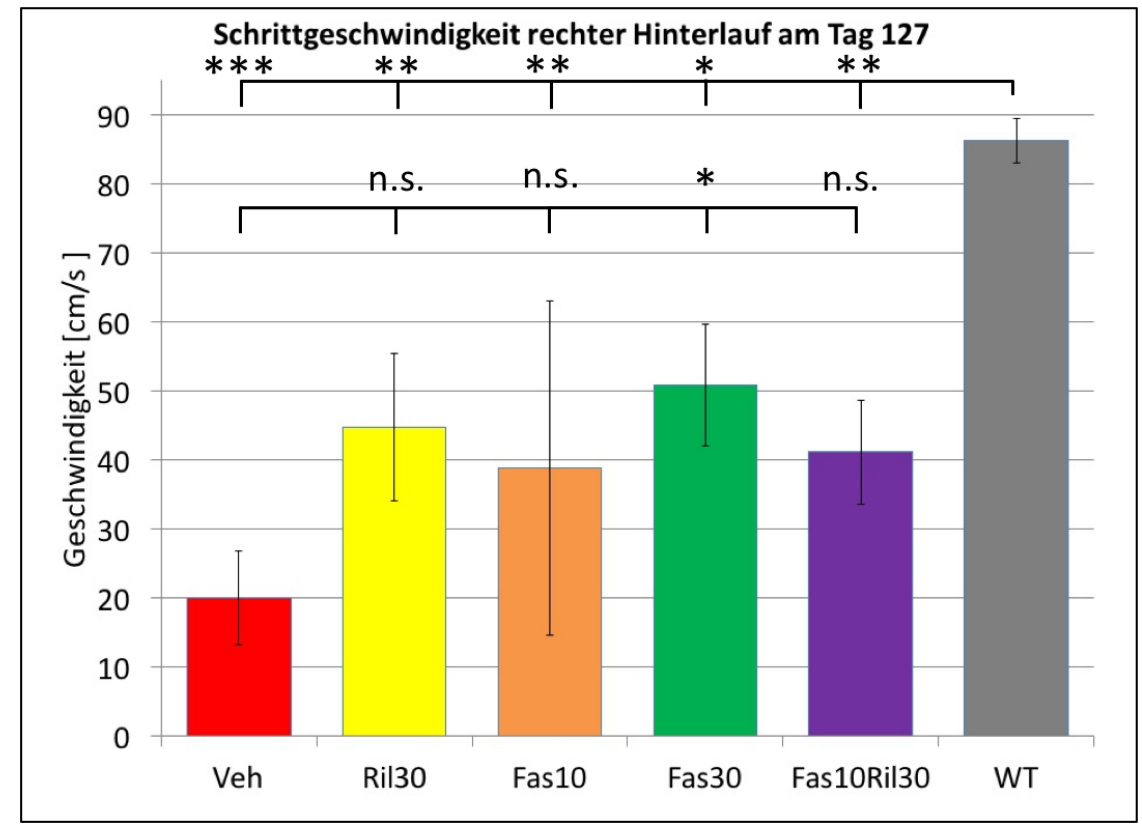

Abbildung 29. Schrittgeschwindigkeit rechter Hinterlauf am Tag 127

Untersucht werden SOD1G93A-transgene weibliche Tiere, die mit Wasser (Veh, n=9), $30 \mathrm{mg} / \mathrm{kg} \mathrm{KG} \mathrm{Riluzol}$ (Ril30, $\mathrm{n}=8$ ), $10 \mathrm{mg} / \mathrm{kg} \mathrm{KG} \mathrm{Fasudil} \mathrm{(Fas10,} \mathrm{n=5),} 30 \mathrm{mg} / \mathrm{kg} \mathrm{KG} \mathrm{Fasudil} \mathrm{(Fas30,} \mathrm{n=7)} \mathrm{und} 10 \mathrm{mg} / \mathrm{kg} \mathrm{KG}$ Fasudil in Kombination mit $30 \mathrm{mg} / \mathrm{kg}$ KG Riluzol (Fas10Ril30, n=7) ab dem Lebenstag 50 behandelt werden. Als gesunde Kontrollgruppe werden 7 SOD1-Wildtyp-Tiere (WT) untersucht. Signifikante Unterschiede zwischen den Gruppen sind bei $\mathrm{p}<0,05$ mit $\left(^{*}\right), \mathrm{p}<0,01$ mit $\left({ }^{* *}\right)$ und $\mathrm{p}<0,001$ mit $\left(^{* * *}\right)$ markiert, n. s. nicht signifikant. Angegeben sind Mittelwerte + - Stdf.

\subsubsection{Schrittlänge (stride length) des rechten Vorderlaufs}

Die Schrittlänge des rechten Vorderlaufs entwickelt sich in allen Gruppen in etwa konstant bis einschließlich zum Tag 99. Ab diesem Zeitpunkt kann eine kontinuierliche Verkürzung der Schrittlänge des rechten Vorderlaufs in der unbehandelten Veh-Kontrollgruppe und den behandelten Gruppen im Gegensatz zur gesunden Wt-Kontrollgruppe beobachtet 
werden. Die gesunden Tiere der Wt-Kontrollgruppe haben über die gesamte Zeit eine konstante dem Gedeihen entsprechende Schrittlänge.

In der ANOVA mit Messwiederholungen aller 12 Untersuchungszeitpunkte zeigt die unbehandelte Veh-Kontrollgruppe einen signifikant schlechteren Verlauf als die gesunde Wt-Kontrollgruppe $(p=0,010)$. Die Behandlungsgruppen zeigen zur unbehandelten VehKontrollgruppen keine signifikante Verbesserung (Ril30: $p=0,974$; Fas10: $p=0,306$; Fas30: $\mathrm{p}=$ 0,771; Fas10Ril30: $\mathrm{p}=0$,335). Im Vergleich zur gesunden Wt-Kontrollgruppe zeigen die Behandlungsgruppen Ril30 ( $\mathrm{p}=0,011)$ und die Fas30-Gruppe $(\mathrm{p}=0,013)$ einen signifikant schlechteren Verlauf. Für die Fas10-Gruppe $(p=0,282)$ und die Fas10Ril30-Gruppe $(\mathrm{p}=0,180)$ gilt dies nicht.

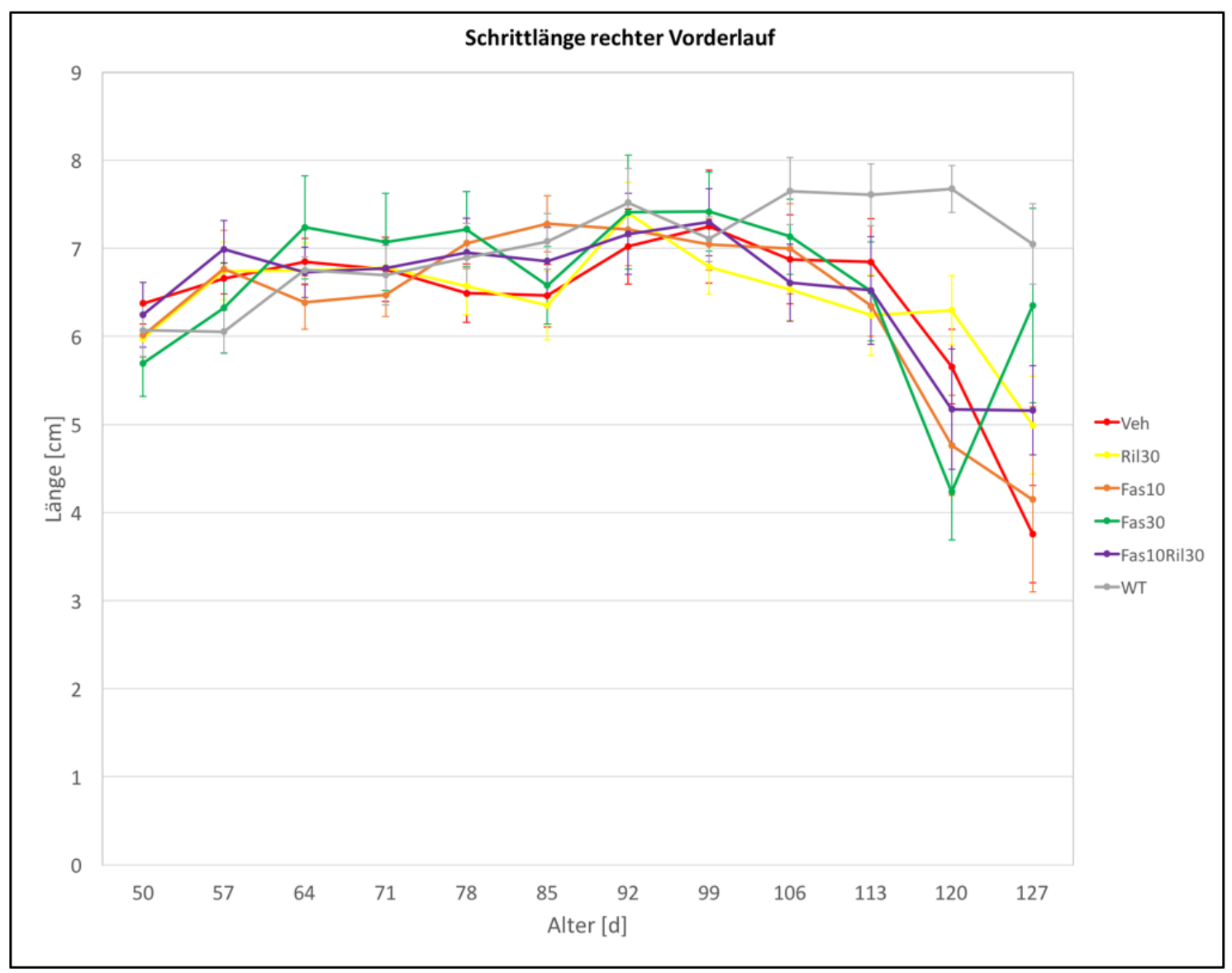

Abbildung 30. Videobasierte Ganganalyse: Schrittlänge rechter Vorderlauf

Untersucht werden SOD1G93A-transgene weibliche Tiere, die mit Wasser (Veh), $30 \mathrm{mg} / \mathrm{kg}$ KG Riluzol (Ril30), 10 mg/kg KG Fasudil (Fas10), 30 mg/kg KG Fasudil (Fas30) und 10 mg/kg KG Fasudil in Kombination mit 30 mg/kg KG Riluzol (Fas10Ril30) ab dem Lebenstag 50 behandelt werden. Als gesunde Kontrollgruppe werden 7 SOD1-Wildtyp-Tiere (WT) untersucht. Anzahl der untersuchten transgenen Tiere je Versuchstag und Gruppe: siehe Tabelle 6. Angegeben sind Mittelwerte +/- Stdf.

Am letzten Untersuchungszeitpunkt, dem Tag 127, kann eine Verkürzung der Schrittlänge des rechten Vorderlaufs in der unbehandelten Veh-Gruppe im Gegensatz zur gesunden Wt-Kontrollgruppe beobachtet werden $(\mathrm{p}=0,001)$. Im Gegensatz zur unbehandelten Veh- 
Kontrollgruppe hat die Fas30-Gruppe $(p=0,002)$ eine signifikant längere Schrittlänge. Der Unterschied der anderen Behandlungsgruppen zur unbehandelten Veh-Kontrollgruppe ist nicht signifikant (Ril30: $p=0,153$; Fas10: $p=0,697$; Fas30: $p=0,771$; Fas10Ril30: $p=0,116$ ).

Die Behandlungsgruppen Ril30 $(p=0,029)$, Fas10 $(p=0,009)$ und Fas10Ril30 $(p=0,050)$ haben eine signifikant kürzere Schrittlänge als die gesunde Wt-Kontrollgruppe. Für die Fas30-Gruppe $(\mathrm{p}=0,471)$ gilt das nicht.

Am Tag 127 beträgt die durchschnittliche Schrittlänge des rechten Vorderlaufes in der unbehandelten Veh-Kontrollgruppe 3,76 +/- 0,55 cm, in der Ril30-Gruppe 4,99 +/- 0,56 $\mathrm{cm}$, in der Fas10-Gruppe 4,15 +/- 1,05 cm, in der Fas30-Gruppe 6,35 +/- 1,1 cm, in der Fas10Ril30-Gruppe 5,16 +/- 0,51 cm, in der gesunden Wt-Kontrollgruppe 7,05 +/- 0,45 $\mathrm{cm}$.

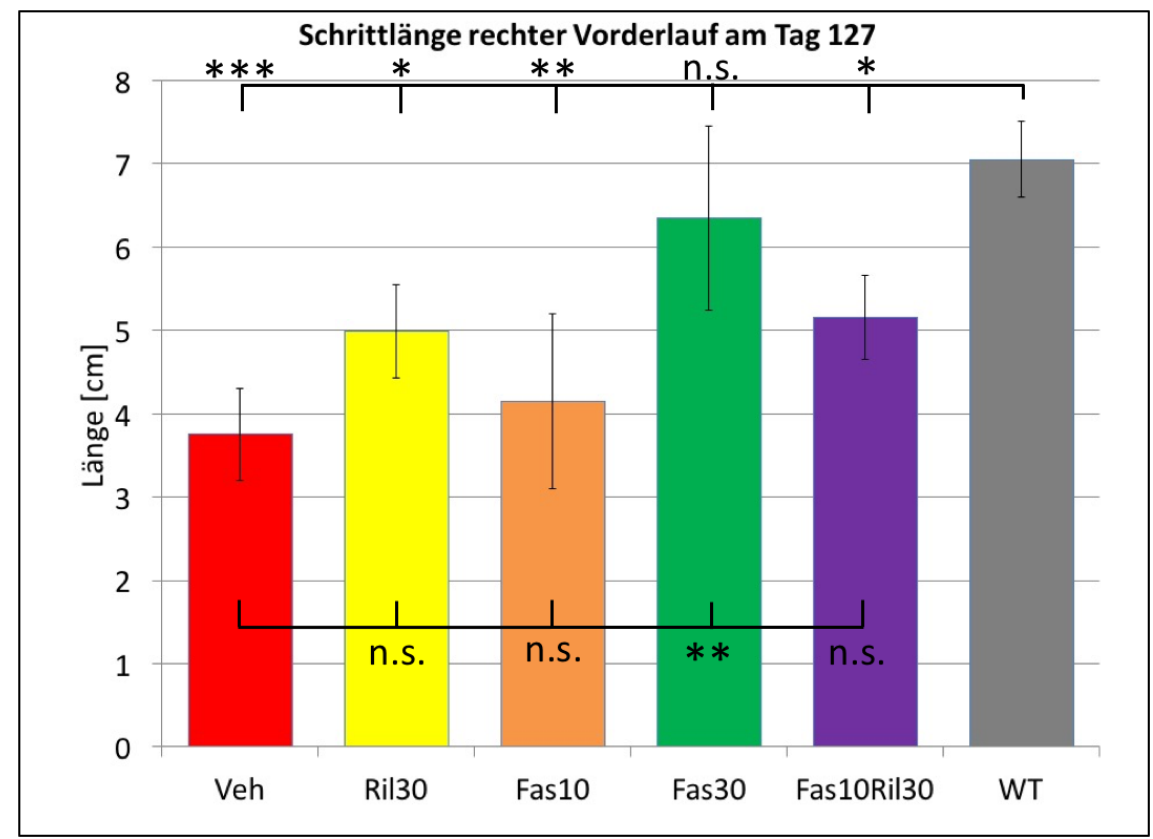

Abbildung 31. Schrittlänge rechter Vorderlauf am Tag 127

Untersucht werden SOD1G93A-transgene weibliche Tiere, die mit Wasser (Veh, n=9), 30 mg/kg KG Riluzol (Ril30, n=8), $10 \mathrm{mg} / \mathrm{kg} \mathrm{KG} \mathrm{Fasudil} \mathrm{(Fas10,} \mathrm{n=5),} 30 \mathrm{mg} / \mathrm{kg} \mathrm{KG} \mathrm{Fasudil} \mathrm{(Fas30,} \mathrm{n=7)} \mathrm{und} 10 \mathrm{mg} / \mathrm{kg}$ KG Fasudil in Kombination mit $30 \mathrm{mg} / \mathrm{kg}$ KG Riluzol (Fas10Ril30, n=7) ab dem Lebenstag 50 behandelt werden. Als gesunde Kontrollgruppe werden 7 SOD1-Wildtyp-Tiere (WT) untersucht. Signifikante Unterschiede zwischen den Gruppen sind bei $\mathrm{p}<0,05$ mit $(*), \mathrm{p}<0,01 \mathrm{mit}\left({ }^{*}\right)$ und $\mathrm{p}<0,001 \mathrm{mit}\left({ }^{* *}\right)$ markiert, n. s. nicht signifikant. Angegeben sind Mittelwerte + /- Stdf.

\subsubsection{Schrittlänge (stride length) des rechten Hinterlaufs}

Es kann eine kontinuierliche Verkürzung der Schrittlänge des rechten Hinterlaufs in der unbehandelten Veh-Kontrollgruppe und den Behandlungsgruppen über den gesamten Untersuchungszeitraum beobachtet werden. Die gesunden Tiere der Wt-Kontrollgruppe haben über die gesamte Zeit eine konstante, dem Gedeihen entsprechende Schrittlänge. 
In der ANOVA mit Messwiederholungen aller 12 Untersuchungszeitpunkte zeigt sich, dass im Verlauf des gesamten Untersuchungszeitraums die Schrittlänge der unbehandelten VehKontrollgruppe signifikant kürzer ist als die der gesunden Wt-Kontrollgruppe $(p=0,010)$. Im Vergleich zur unbehandelten Veh-Kontrollgruppe zeigen die Behandlungsgruppen keinen signifikanten Unterscheid (Ril30: $p=0,823$; Fas10: $p=0,140$; Fas30: $p=0,206$; Fas10Ril30: p=0,409). Im Vergleich zur gesunden Wt-Kontrollgruppe zeigt die Ril30Gruppe $(p=0,016)$ und auch die Fas30-Gruppe $(p=0,001)$ im Gesamtzeitraum eine signifikant langsamere Schrittlänge des rechten Hinterlaufs. Ansonsten zeigen sich keine signifikanten Unterschiede zur gesunden Wt-Kontrollgruppe (Fas10: p=0,527; Fas10Ril30: $\mathrm{p}=0,135)$.

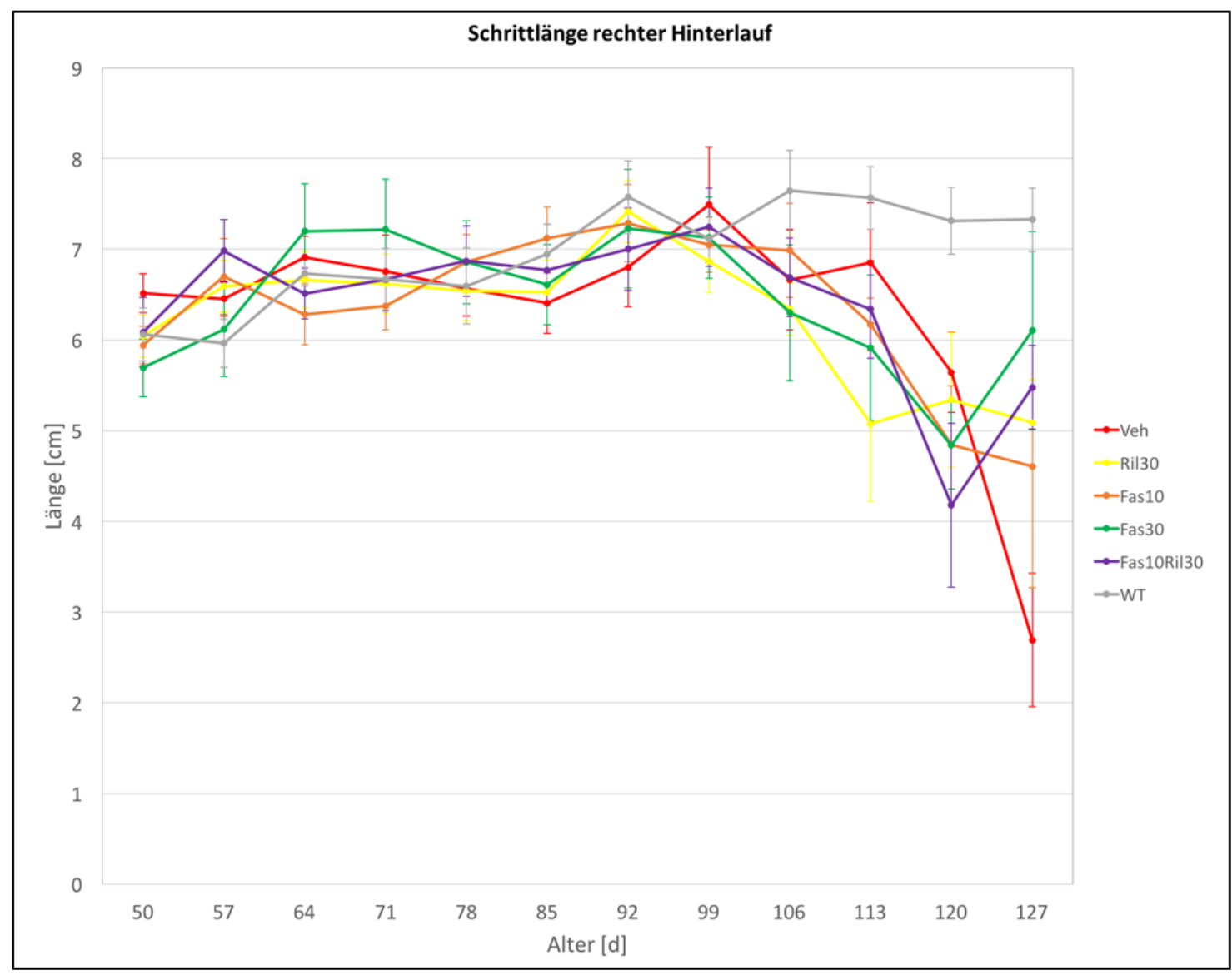

Abbildung 32. Videobasierte Ganganalyse: Schrittlänge rechter Hinterlauf

Untersucht werden SOD1G93A-transgene weibliche Tiere, die mit Wasser (Veh), 30 mg/kg KG Riluzol (Ril30), 10 mg/kg KG Fasudil (Fas10), 30 mg/kg KG Fasudil (Fas30) und 10 mg/kg KG Fasudil in Kombination mit 30 mg/kg KG Riluzol (Fas10Ril30) ab dem Lebenstag 50 behandelt werden. Als gesunde Kontrollgruppe werden 7 SOD1-Wildtyp-Tiere (WT) untersucht. Anzahl der untersuchten transgenen Tiere je Versuchstag und Gruppe: siehe Tabelle 6. Angegeben sind Mittelwerte +/- Stdf.

Am letzten Untersuchungszeitpunkt, dem Tag 127, kann eine Verkürzung der Schrittlänge des rechten Hinterlaufs in der unbehandelten Veh-Gruppe im Gegensatz zur gesunden WtKontrollgruppe beobachtet werden $(\mathrm{p}=0,000)$. Im Gegensatz zur unbehandelten Veh- 
Kontrollgruppe haben die Ril30-Gruppe $(p=0,019)$, die Fas30-Gruppe $(p=0,002)$ und die Fas10Ril30 ( $p=0,009)$ eine signifikant längere Schrittlänge. Der Unterschied der Fas10Gruppe zur unbehandelten Veh-Kontrollgruppe ist nicht signifikant $(\mathrm{p}=0,098)$.

Die Behandlungsgruppen Ril30 ( $p=0,039)$ und Fas10 ( $p=0,027)$ haben ebenso wie die unbehandelte Veh-Kontrollgruppe eine signifikant kürzere Schrittlänge als die gesunde WtKontrollgruppe. Für die Fas30-Gruppe $(p=0,265)$ und die Fas10Ril30-Gruppe $(p=0,095)$ gilt das nicht.

Am Tag 127 beträgt die durchschnittliche Schrittlänge des rechten Hinterlaufes in der unbehandelten Veh-Kontrollgruppe 2,69 +/- 0,74 cm, in der Ril30-Gruppe 5,09 +/- 0,47 $\mathrm{cm}$, in der Fas10-Gruppe 4,6 +/- 1,34 cm, in der Fas30-Gruppe 6,11 +/- 1,09 cm, in der Fas10Ril30-Gruppe 5,48 +/- 0,46 cm, in der gesunden Wt-Kontrollgruppe 7,32 +/- 0,34 $\mathrm{cm}$.

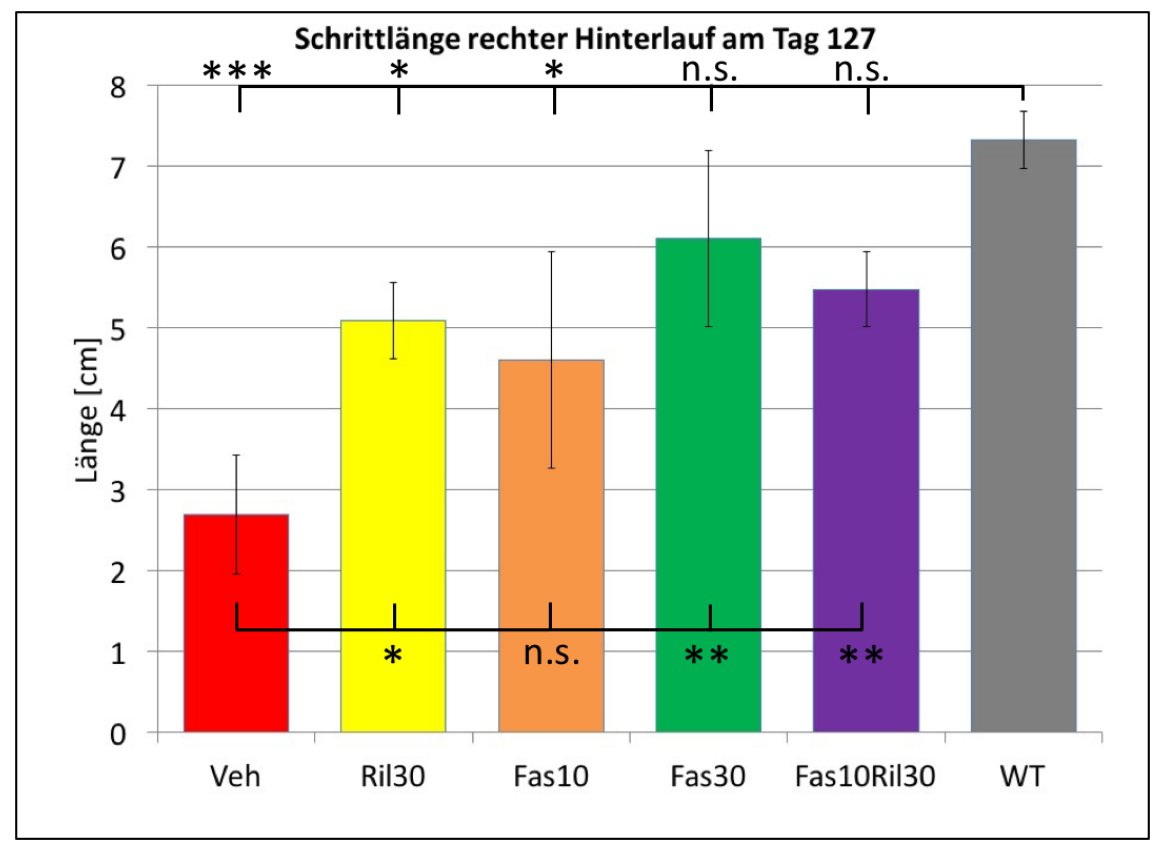

Abbildung 33. Schrittlänge rechter Hinterlauf am Tag 127

Untersucht werden SOD1G93A-transgene weibliche Tiere, die mit Wasser (Veh, n=9), 30 mg/kg KG Riluzol (Ril30, $\mathrm{n}=8$ ), $10 \mathrm{mg} / \mathrm{kg} \mathrm{KG}$ Fasudil (Fas10, n=5), $30 \mathrm{mg} / \mathrm{kg} \mathrm{KG} \mathrm{Fasudil} \mathrm{(Fas30,} \mathrm{n=7)} \mathrm{und} 10 \mathrm{mg} / \mathrm{kg}$ KG Fasudil in Kombination mit $30 \mathrm{mg} / \mathrm{kg}$ KG Riluzol (Fas10Ril30, n=7) ab dem Lebenstag 50 behandelt werden. Als gesunde Kontrollgruppe werden 7 SOD1-Wildtyp-Tiere (WT) untersucht. Signifikante Unterschiede zwischen den Gruppen sind bei $\mathrm{p}<0,05$ mit $(*), \mathrm{p}<0,01$ mit $\left(^{* *}\right)$ und $\mathrm{p}<0,001$ mit $\left(^{* * *}\right)$ markiert, n. s. nicht signifikant. Angegeben sind Mittelwerte + /- Stdf.

\subsubsection{Kognitiver Test (novel object recognition test)}

Eingeschlossen werden die Tiere aus der Verlaufsstudie. Es werden in den Gruppen Veh, Ril30, Fas10, Fas30 und Fas10Ril30 pro Gruppe und pro Versuchstag mindestens 9, maximal 12 Videos in die Auswertung erstellt und ausgewertet, lediglich am Lebenstag 102 können in der Fas10-Gruppe nur 6 Videos erstellt und ausgewertet werden. In der Wt- 
Gruppe wurden je Versuchstag 7 Videos erstellt und ausgewertet (Tabelle 7). Die Videos wurden mithilfe der Computersoftware Ethovision XT 8.5 (Noldus, Wageningen, Niederlande) ausgewertet. Dabei werden einerseits die zurückgelegte Strecke als Parameter und der aus den Erkundungszeiten zu errechnende discrimination index (DI) gewählt.

\begin{tabular}{|l|c|c|c|c|}
\hline Untersuchungstag & $\mathbf{5 0}$ & $\mathbf{8 8}$ & $\mathbf{1 0 2}$ & $\mathbf{1 1 6}$ \\
\hline Veh & 11 & 9 & 9 & 10 \\
\hline Ri130 & 12 & 9 & 9 & 9 \\
\hline Fas10 & 11 & 9 & 6 & 9 \\
\hline Fas30 & 12 & 10 & 9 & 10 \\
\hline Fas10Ri130 & 12 & 9 & 9 & 7 \\
\hline Wt & 7 & 7 & 7 & 9 \\
\hline
\end{tabular}

Tabelle 7. Kognitiver Test: Anzahl aufgenommener und ausgewerteter Videos

Die zurückgelegte Strecke dient dabei als Maß für das generelle Explorationsverhalten der Tiere: Am Lebenstag 50 unterscheidet sich die im Mittel zurückgelegte Strecke in den verschiedenen Gruppen nicht signifikant voneinander (Abbildung 37 A). Im Einzelnen beträgt die im Mittel zurückgelegte Strecke in der Veh-Gruppe 2085,09 +/- 522,39 cm, in der Ril30-Gruppe 1668,14 +/- 222,51 cm, in der Fas10-Gruppe 1818,15 +/- 544,91 cm, in der Fas30-Gruppe 1235,39 +/-378,64 cm, in der Fas10Ril30-Gruppe 1399,79 +/- 325,36 $\mathrm{cm}$, in der Wt-Gruppe 850,59+/- 236,35 cm.

Am Lebenstag 88 unterscheidet sich die im Mittel zurückgelegte Strecke in den verschiedenen Gruppen nicht signifikant voneinander (Abbildung 37 B). Im Einzelnen beträgt die im Mittel zurückgelegte Strecke in der Veh-Gruppe 2235,51 +/- 546,27 cm, in der Ril30-Gruppe 1246,06 +/- 203,23 cm, in der Fas10-Gruppe 1616,07 +/- 526,82 cm, in der Fas30-Gruppe 2080,72 +/- 478,65 cm, in der Fas10Ril30-Gruppe 1663,66 +/- 242,88 $\mathrm{cm}$, in der Wt-Gruppe 909,57 +/- 264,45 cm.

Am Lebenstag 102 legen die Tiere der Ril30-Gruppe im Mittel signifikant mehr Strecke zurück als die Tiere der Veh-Gruppe. Ansonsten unterscheidet sich die im Mittel zurückgelegte Strecke in den verschiedenen Gruppen nicht signifikant voneinander (Abbildung 37 C). Im Einzelnen beträgt die im Mittel zurückgelegte Strecke in der VehGruppe 974,76 +/- 325,51 cm, in der Ril30-Gruppe 1900,5 +/- 324,12 cm, in der Fas10Gruppe 1530,85 +/- 424,08 cm, in der Fas30-Gruppe 1687,76 +/- 482,08 cm, in der Fas10Ril30-Gruppe 1052,61 +/- 329,76 cm, in der Wt-Gruppe 1887,51 +/- 452,82 cm. 
Am Lebenstag 116 legen die Tiere der Fas30-Gruppe im Mittel signifikant mehr Strecke zurück als die Tiere der Fas10Ril30-Gruppe. Ansonsten unterscheidet sich die im Mittel zurückgelegte Strecke in den verschiedenen Gruppen nicht signifikant voneinander (Abbildung 37 D). Im Einzelnen beträgt die zurückgelegte Strecke im Mittel in der VehGruppe 1304,82 +/- 389,75 cm, in der Ril30-Gruppe 1396,83 +/- 301,17 cm, in der Fas10Gruppe 1075,98 +/- 345,13 cm, in der Fas30-Gruppe 1502,86 +/- 278,69 cm, in der Fas10Ril30-Gruppe 594,81 +/- 120,27 cm, in der Wt-Gruppe 1545,45 +/- 488,83 cm.

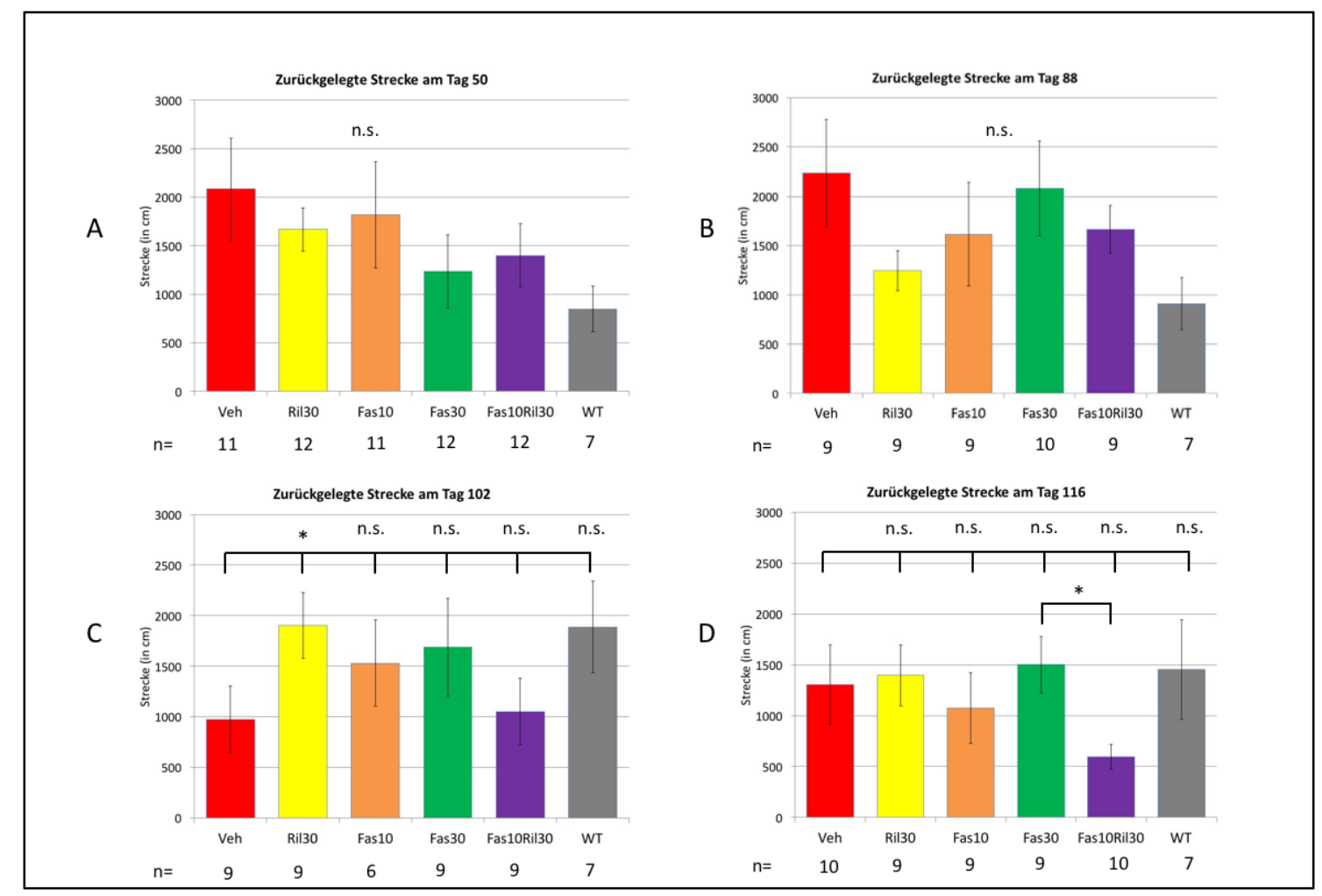

Abbildung 34. Kognitiver Test (novel object recognition test): Zurückgelegte Strecke

Untersucht werden am Lebenstag 50 (A), am Lebenstag 88 (B), am Lebenstag 102 (C) und am Lebenstag 116 (D) SOD1G93A-transgene weibliche Tiere, die mit Wasser (Veh), 30 mg/kg KG Riluzol (Ril30), 10 mg/kg KG Fasudil (Fas10), $30 \mathrm{mg} / \mathrm{kg} \mathrm{KG} \mathrm{Fasudil} \mathrm{(Fas30)} \mathrm{und} 10 \mathrm{mg} / \mathrm{kg} \mathrm{KG} \mathrm{Fasudil} \mathrm{in} \mathrm{Kombination} \mathrm{mit} 30 \mathrm{mg} / \mathrm{kg} \mathrm{KG} \mathrm{Riluzol}$ (Fas10Ril30) ab dem Lebenstag 50 behandelt werden. Als gesunde Kontrollgruppe werden 7 SOD1-Wildtyp-Tiere (WT) untersucht. Signifikante Unterschiede zwischen den Gruppen sind bei p<0,05 mit (*) markiert, n. s. nicht signifikant. Angegeben sind Mittelwerte +/- Stdf. Als n angegeben ist jeweils die Anzahl der in die Auswertung mit einbezogenen Videos (siehe Tabelle 7).

Als Parameter für die kognitive Leistungsfähigkeit dient der DI: Aus den Erkundungszeiten der Maus an dem bekannten und dem unbekannten Objekt lässt sich der discrimination index errechnen (Antunes und Biala 2012). Er eribt sich aus der Differenz der verbrachten Zeit beim unbekannten minus der beim bekannten Objekt dividiert durch die Gesamtzeit, die bei den Objekten verbracht wurde. Dabei ergeben sich dimensionslose Werte zwischen -1 und 1. Ein hoher Wert spricht für eine gute Diskriminationsverhalten und eine gute kognitive Leistungsfähigkeit der Mäuse, ein niedriger Wert dafür, dass das neue Objekt 
nicht als ein solches wahrgenommen wurde. Da an jedem unterschiedlichen Versuchstag verschiedene Objekte verwendet werden, kann eine Vergleichbarkeit zwischen den Tagen nicht gewährleistet werden, da bestimmte Objekte möglicherweise eine größere Anziehung auf die Tiere ausüben als andere und die Eigenschaft des Unbekanntseins, die für die Diskrimination zum alten Objekt nötig ist, nur für das jeweils unbekannte Objekt gelten kann. Daher werden die Werte nur am einzelnen Versuchstag mittels einer univarianten ANOVA auf Unterschiede untersucht:

Der discrimination index unterscheidet sich in den Gruppen an keinem gewählten Untersuchungszeitpunkt signifikant voneinander.

Am Lebenstag 50 beträgt der discrimination index im Mittel in der Veh-Gruppe 0,17 +/0,14; in der Ril30-Gruppe 0,36 +/- 0,21; in der Fas10-Gruppe 0,31 +/- 0,15; in der Fas30Gruppe -0,02 +/- 0,12; in der Fas10Ril30-Gruppe 0,12 +/- 0,17; in der Wt-Gruppe 0,18 $+/-0,15$.

Am Lebenstag 88 beträgt der discrimination index im Mittel in der Veh-Gruppe -0,25 +/0,21; in der Ril30-Gruppe -0,23 +/- 0,19; in der Fas10-Gruppe -0,07 +/- 0,2; in der Fas30Gruppe 0,21 +/- 0,16; in der Fas10Ril30-Gruppe 0,1 +/- 0,24; in der Wt-Gruppe -0,02 $+/-0,3$.

Am Lebenstag 102 beträgt der discrimination index im Mittel in der Veh-Gruppe -0,09 +/0,14; in der Ril30-Gruppe 0,01 +/- 0,22; in der Fas10-Gruppe -0,4 +/- 0,22; in der Fas30Gruppe 0,03 +/- 0,18; in der Fas10Ril30-Gruppe 0,21 +/- 0,2; in der Wt-Gruppe -0,13 $+/-0,13$.

Am Lebenstag 116 beträgt der discrimination index im Mittel in der Veh-Gruppe 0,3 +/0,11; in der Ril30-Gruppe -0,02 +/- 0,25; in der Fas10-Gruppe 0,26 +/- 0,13; in der Fas30Gruppe 0,43 +/- 0,17; in der Fas10Ril30-Gruppe 0,1 +/- 0,15; in der Wt-Gruppe 0,19 +/0,19 . 


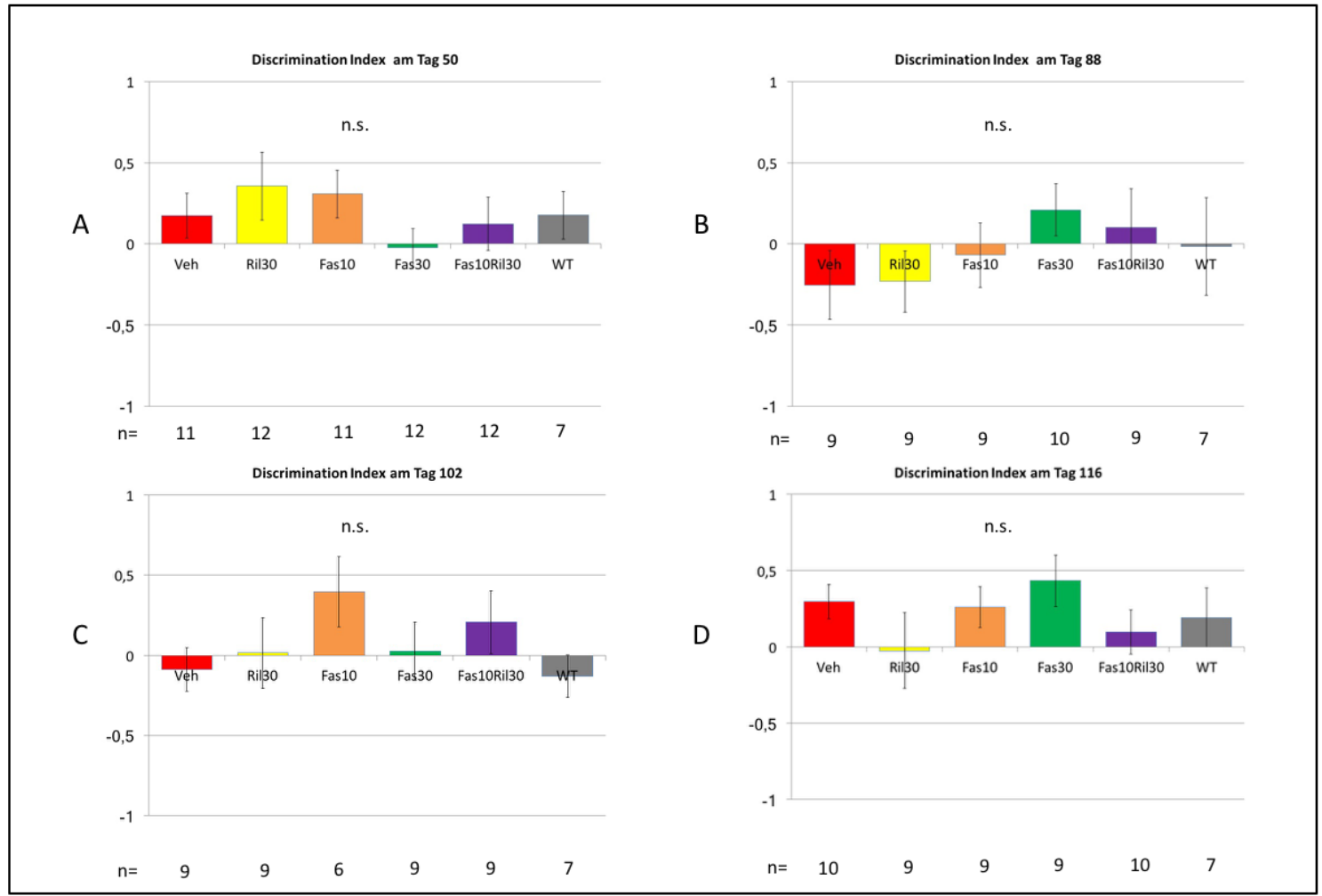

Abbildung 35. Kognitiver Test (novel object recognition test): discrimination index

Untersucht werden am Lebenstag 50 (A), am Lebenstag 88 (B), am Lebenstag 102 (C) und am Lebenstag 116 (D) SOD1G93A-transgene weibliche Tiere, die mit Wasser (Veh), $30 \mathrm{mg} / \mathrm{kg}$ KG Riluzol (Ril30), $10 \mathrm{mg} / \mathrm{kg}$ KG Fasudil (Fas10), $30 \mathrm{mg} / \mathrm{kg} \mathrm{KG} \mathrm{Fasudil} \mathrm{(Fas30)} \mathrm{und} 10 \mathrm{mg} / \mathrm{kg}$ KG Fasudil in Kombination mit $30 \mathrm{mg} / \mathrm{kg}$ KG Riluzol (Fas10Ril30) ab dem Lebenstag 50 behandelt werden. Als gesunde Kontrollgruppe werden 7 SOD1-Wildtyp-Tiere (WT) untersucht. Die Unterschiede zwischen den Gruppen sind nicht signifikant (n. s.). Angegeben sind Mittelwerte +/- Stdf. Als $\mathrm{n}$ angegeben ist jeweils die Anzahl der in die Auswertung mit einbezogenen Videos (siehe Tabelle 7). 


\subsection{Querschnittstudie nach präsymptomatischer Behandlung mit Fasudil und Riluzol ab Lebenstag 50}

Es wird eine Querschnittsanalyse der lumbalen Alpha-Motoneuronenanzahl am 120. Lebenstag durchgeführt, um die Wirkung in den verschiedenen Behandlungsgruppen zu untersuchen. Außerdem wird das Ausmaß der Muskelatrophie des M. gastrocnemius evaluiert. Die Tiere werden im Rahmen einer präsymptomatischen Behandlung ab dem 50. Lebenstag oral behandelt. Eine gesunde Wt-Gruppe und eine unbehandelte transgene VehGruppe dienen als Kontrolle. Am 120. Lebenstag werden den euthanasierten Tieren das Rückenmark für die immunhistochemische Analyse und der M. gastrocnemius zur Atrophie-Bestimmung entnommen.

\subsubsection{Evaluation der Atrophie des Musculus gastrocnemius}

Es werden jeweils 5 Tiere aus den 4 Behandlungsgruppen (Fas 10, Fas30, Ril30, Fas10Ril30) und aus der Veh-Gruppe, sowie 10 Tiere aus der Wt-Gruppe analysiert. Evaluiert wird der prozentuale Anteil des M. gastrocnemius am Körpergewicht.

Dieser ist an Tag 120 im unbehandelten transgenen Tier im Vergleich zum Wildtyptier signifikant vermindert (Wt: 0,57\% +/- 0,02; Veh: 0,36\% +/- 0,01). Der prozentuale Verlust an Muskelmasse im Vergleich zum gesunden Tier beträgt somit 36,84\%.

Im Vergleich zur unbehandelten Veh-Gruppe ist der prozentuale Anteil am Körpergewicht des Musculus gastrocnemius in den Behandlungsgruppen Ril30 (0,51\% +/- 0,03), Fas10 $(0,52 \%+/-0,03)$ und Fas30 (0,49\% +/-0,03) signifikant erhöht. Der Muskelanteil der Tiere aus der Ril30-Gruppe und der Fas10-Gruppe unterscheidet sich nicht signifikant von den gesunden Tieren. Hier lässt sich ein prozentualer Verlust von 10,52\% bzw. 8,77 \% zeigen. Im Vergleich zur gesunden Wt-Kontrollgruppe haben neben der unbehandelten Veh-Kontrollgruppe auch die Fas30-Gruppe und die Fas10Ril30-Gruppe ein signifikant geringeren Muskelanteil am Körpergewicht. Hier beträgt der prozentuale Verlust im Vergleich zum gesunden Tier 14,04\% bzw. 26,32\%. Es kann keine signifikante Erhöhung des prozentualen Anteils am Körpergewicht bei Tieren unter der Kombinationsbehandlung Fas10Ril30 festgestellt werden (Fas10Ril30: 0,42\% +/- 0,04). 


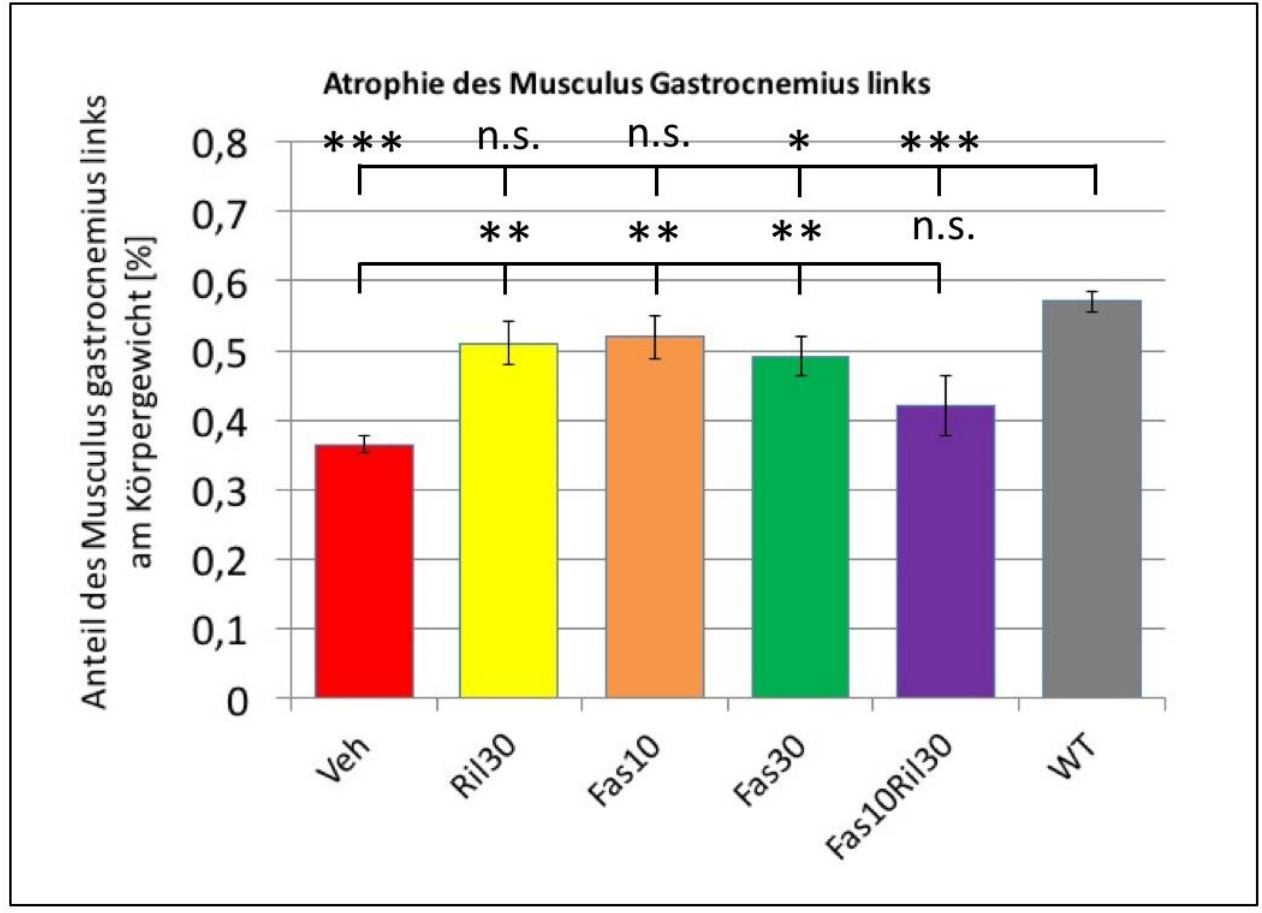

Abbildung 36. Anteil des Musculus gastrocnemius am Körpergewicht an Lebenstag 120

Untersucht werden pro Gruppe 5 weibliche SOD1G93A-transgene Tiere, die mit Wasser (Veh), 30 mg/kg KG Riluzol (Ril30), 10 mg/kg KG Fasudil (Fas10), 30 mg/kg KG Fasudil (Fas30) und 10 mg/kg KG Fasudil in Kombination mit 30 $\mathrm{mg} / \mathrm{kg}$ KG Riluzol (Fas10Ril30) ab dem Lebenstag 50 behandelt werden. Als gesunde Kontrollgruppe werden 10 SOD1Wildtyp-Tiere (WT) untersucht. Signifikante Unterschiede zwischen den Gruppen sind bei $\mathrm{p}<0,05$ mit $\left(^{*}\right), \mathrm{p}<0,01$ mit $\left(^{* *}\right)$ und $\mathrm{p}<0,001$ mit $(* * *)$ markiert, n. s. nicht signifikant. Angegeben sind Mittelwerte +/- Stdf.

\subsubsection{Histologische Evaluation der spinalen Alpha-Motoneurone}

Es werden jeweils 5 Tiere aus den 4 Behandlungsgruppen (Fas 10, Fas30, Ril30, Fas10Ril30), aus der Veh-Gruppe und aus der Wt-Gruppe untersucht. Die Gesamtzahl der Alpha-Motoneurone pro Vorderhorn im lumbalen Rückenmark ist an Tag 120 im unbehandelten Tier im Vergleich zum Wildtyptier signifikant vermindert (Wt: 10,56 +/2,72 Motoneurone; Veh: 2,74 +/- 0,5).

Ebenfalls ist die Gesamtzahl der Motoneurone in den Fasudil-Gruppen signifikant zum Wildtyptier vermindert (Fas10: 3,36 +/- 1,12; Fas30: 3,25 +/-0,92). Auch in den mit Riluzol und mit der Kombination behandelten Gruppen ist die Gesamtzahl signifikant zum Wildtyptier vermindert (Ril30: 4,33 +/-0,8; Fas10Ril30: 3,84 +/- 0,59).

Zwischen den Behandlungsgruppen und der Veh-Gruppe lassen sich keine signifikanten Änderungen feststellen (Fas10, Fas10Ril30, Fas30, Ril30, Veh). Die Unterschiede zwischen den Behandlungsgruppen sind ebenso nicht signifikant. 


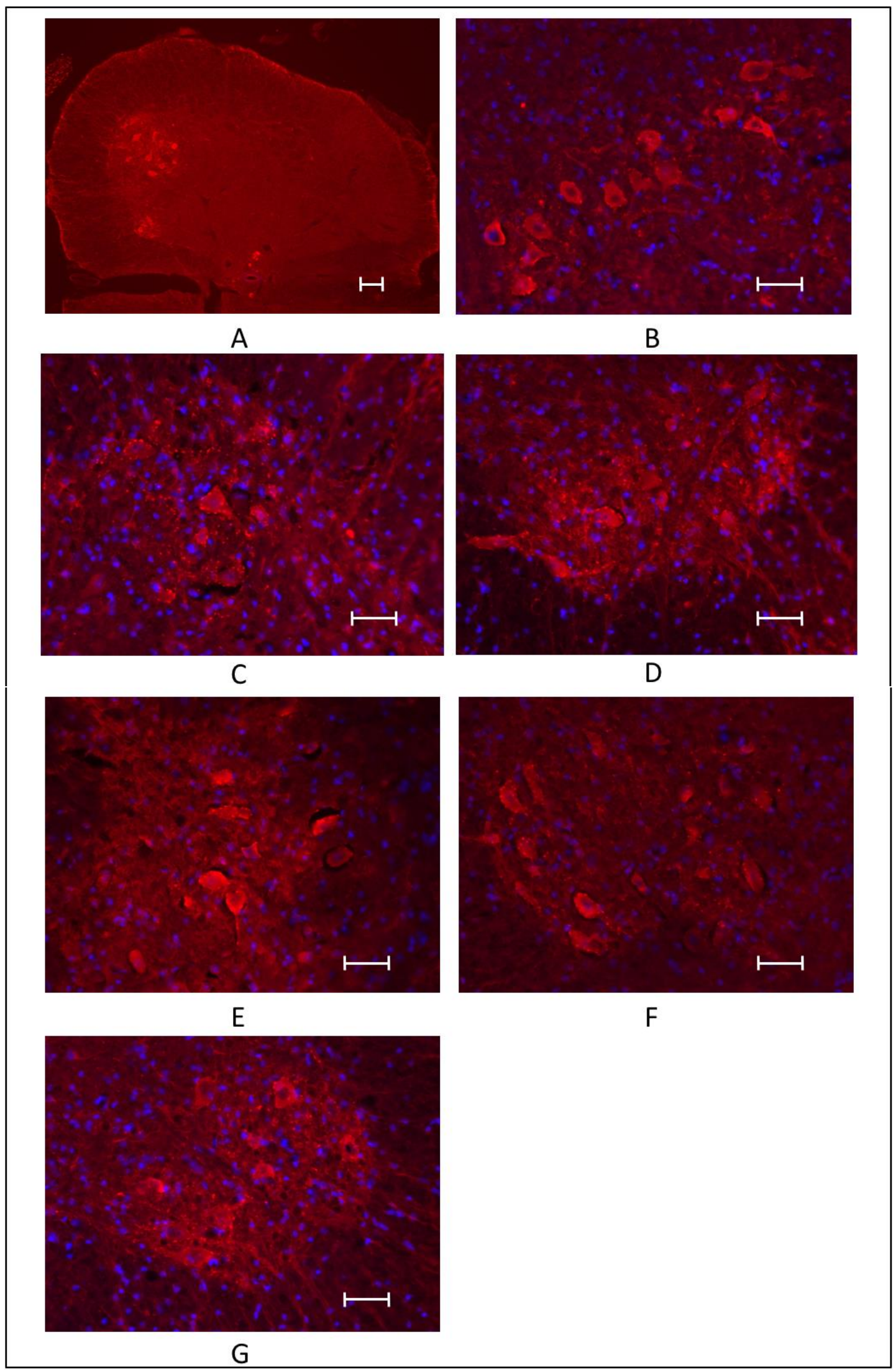

Abbildung 37. ChAT-Staining: Anzahl der Alpha-Motoneurone/Vorderhorn im Lumbalmark

In 5-facher Vergrößerung mit Cy3 (rot) die Übersicht über eine Seite eines Rückenmarksquerschnitts eines Tieres der WtGruppe (A) mit gut erkennbaren ChAT-positiven Zellen, Maßstabbalken $100 \mu \mathrm{m}$. In 20-facher Vergrößerung mit Cy3 (rot) und DAPI (blau) die Alpha-Motoneurone im Vorderhorn eines Tieres der Wt-Gruppe (B), der Veh-Gruppe (C) der Ril30-Gruppe (D), der Fas10-Gruppe (E), der Fas30-Gruppe (F) und der Fas10Ril30-Gruppe (G), Maßstabbalken $50 \mu \mathrm{m}$. 


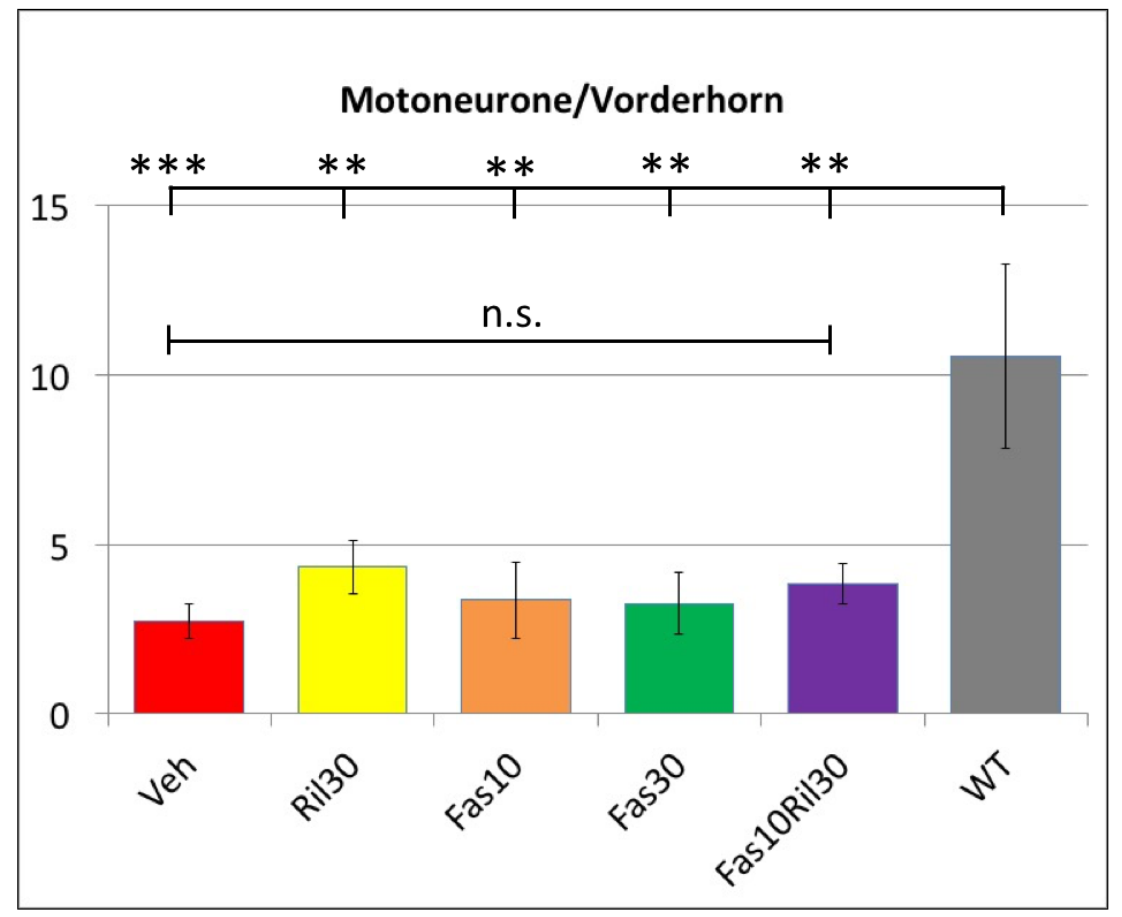

Abbildung 38. Alpha-Motoneurone/Vorderhorn am Lebenstag 120

Untersucht werden pro Gruppe 5 weibliche SOD1G93A-transgene Tiere, die mit Wasser (Veh), 30 mg/kg KG Riluzol (Ril30), 10 mg/kg KG Fasudil (Fas10), 30 mg/kg KG Fasudil (Fas30) und 10 mg/kg KG Fasudil in Kombination mit 30 $\mathrm{mg} / \mathrm{kg} \mathrm{KG} \mathrm{Riluzol} \mathrm{(Fas10Ril30)} \mathrm{ab} \mathrm{dem} \mathrm{Lebenstag} 50$ behandelt werden. Als gesunde Kontrollgruppe werden 5 SOD1Wildtyp-Tiere (WT) untersucht. Signifikante Unterschiede zwischen den Gruppen sind bei $\mathrm{p}<0,01$ mit $(* *)$ und $\mathrm{p}<0,001$ mit (***) markiert, n. s. nicht signifikant. Angegeben sind Mittelwerte +/- Stdf. 


\section{Diskussion}

Ziel dieser Promotionsarbeit ist es, den Einfluss von Fasudil in zwei unterschiedlichen Dosierungen (10 bzw. $30 \mathrm{mg} / \mathrm{kg} \mathrm{KG}$ ) sowie in einer Kombinationsbehandlung mit Riluzol auf die Erkrankung SOD1G93A-transgener ALS-Mäuse zu untersuchen. Als Kontrollgruppe dient der gesunde SOD1-Wildtyp und unbehandelte SOD1G93Atransgene Mäuse. Der Therapiebeginn ist präsymptomatisch an Lebenstag 50.

Zur Untersuchung der Effekte auf verhaltensbiologische Parameter wird einerseits eine Verlaufsstudie durchgeführt. Dabei wird der der Einfluss auf folgende Parameter untersucht: das Überleben, die klinische Entwicklung anhand des Gewichts und eines klinisch-neurologischen Scores, auf die motorische Performance mittels Drehwalzenversuch (Rotarod) und mittels videobasierter Ganganalyse (CatWalk XT) und auf die kognitive Entwicklung mittels novel object recognition test (NOR-Test).

Andererseits wird eine Querschnittstudie zur histologischen Untersuchung der Anzahl lumbaler Alpha-Motoneurone und der makroskopischen Untersuchung der Atrophie des Musculus gastrocnemius am Lebenstag 120 durchgeführt.

\subsection{Verlaufsstudie unter präsymptomatischer Behandlung mit Fasudil und Riluzol}

\subsubsection{Kein Überlebensvorteil - Applikationsdauer von Bedeutung}

Die Verlaufsstudie unter präsymptomatischer Behandlung mit Fasudil in zwei Dosierungen, $10 \mathrm{mg} / \mathrm{kg} \mathrm{KG}$ und $30 \mathrm{mg} / \mathrm{kg}$, und Riluzol (30 mg/kg KG) sowie einer Kombination beider Präparate, wobei Fasudil in der niedrigeren Dosierung verabreicht wurde, dient dazu, einen möglichen Überlebensvorteil der gewählten Therapie zu untersuchen.

Sobald die Versuchsabbruchkriterien (siehe Kapitel 2.7.1.1) erfüllt sind, werden die Tiere euthanasiert. Der Tag des Versuchsabbruchs wird als Endpunkt in die Auswertung mittels Kaplan-Meier-Schätzer aufgenommen.

Takata et al. (2013) können in einer Verlaufsstudie, in der Tiere des SOD1G93AMausmodells ab dem 35. Lebenstag behandelt werden, ein verlängertes Überleben durch eine Behandlung mit Fasudil $30 \mathrm{mg} / \mathrm{kg} \mathrm{KG}$ und mit $100 \mathrm{mg} / \mathrm{kg} \mathrm{KG}$ zeigen. Auch im Rahmen unserer Forschungsgruppe können wir in einer vorherigen Studie einen Überlebensvorteil für Tiere des SOD1G93A-Mausmodells beobachten, die ab dem 50 . 
Lebenstag mit Fasudil (30 mg/kg KG) behandelt werden (Tönges et al. 2014). In der vorliegenden Studie mittels log-Rank-Test zeigt sich für Fasudil (30 mg/kg KG) nur ein Trend zu einem verbesserten Überleben. Die anderen Therapieregimes sind bzgl. Überleben nicht vorteilhaft. Günther (2015) kann bei einem symptomatischen Behandlungsbeginn am Lebenstag 80 ebenfalls keinen Überlebensvorteil für Fasudil (30 $\mathrm{mg} / \mathrm{kg} \mathrm{KG)} \mathrm{beobachten.}$

So lässt sich schließen, dass ein frühzeitiger Behandlungsbeginn und eine damit einhergehende längere Applikationsdauer die Wirksamkeit von Fasudil und vor allem dessen Auswirkung auf das Überleben der transgenen Tiere des SOD1G93A-Mausmodells maßgeblich erhöht.

Zusammenfassen lassen sich die Erkenntnisse wie folgt:

1. Riluzol (30 mg/kg KG), Fasudil (10 mg/kg KG), Fasudil (30 mg/kg KG), Fasudil (10 mg/kg KG) in Kombination mit Riluzol (30 mg/kg KG) können in der vorliegenden Studie mit einem Behandlungsbeginn am Lebenstag 50 das Überleben nicht signifikant verlängern.

2. Andere Studien können einen Überlebensvorteil für Fasudil (30 mg/kg KG und $100 \mathrm{mg} / \mathrm{kg} \mathrm{KG}$ ) nachweisen (Tönges et al. 2014, Takata et al. 2013). Im Zusammenhang mit weiteren Studien (Günther 2015) kann man schließen, dass dabei ein früherer Behandlungsbeginn (Takata et al. 2013) und eine längere Applikationsdauer die neuroprotektive Wirksamkeit mit Überlebensvorteil von Fasudil begünstigt.

\subsubsection{Kein modifizierender Einfluss auf den Krankheitsverlauf}

Im Rahmen der Verlaufsstudie wird die klinische Symptomatik evaluiert, um einen Einfluss der Therapie beurteilen zu können. Dazu dient der klinisch-neurologische Score nach Weydt et al. (2003), der in unserer Forschungsgruppe auch in vorherigen Studien genutzt wurde und gut etabliert ist (siehe Kapitel 2.7.1.1). Wichtigster Parameter sind Tag des Krankheitsbeginns, d. h. erstmaliges Beobachten klinischer Symptome (Score 3) und die Krankheitsdauer, gemessen von Krankheitsbeginn bis zum Tod. Das Fortschreiten und die Ausbreitung der Symptome werden mit weiteren Abstufungen im Score dokumentiert und auch hier der Tag des erstmaligen Auftretens ermittelt.

Takata et al. (2013) beobachten eine signifikante Verzögerung des Krankheitsbeginns um fast $10 \%$ durch eine Therapie mit Fasudil in den Dosierungen $30 \mathrm{mg} / \mathrm{kg} \mathrm{KG}$ und 100 $\mathrm{mg} / \mathrm{kg}$ KG bei einem Therapiebeginn am Lebenstag 35 und einem mittleren Krankheitsbeginn der unbehandelten Kontrollgruppe um den Lebenstag 109. Der 
Krankheitsbeginn wird hier jedoch nicht klinisch ermittelt, sondern durch erstmaliges Versagen beim Drehwalzenversuch (Rotarod) definiert. Die Tiere werden bei einer konstanten Geschwindigkeit $600 \mathrm{~s}$ beobachtet und ein vorzeitiges Herunterfallen als Versagen definiert. Der Effekt auf den Krankheitsbeginn kann in unserer Forschungsgruppe, die den Krankheitsbeginn rein klinisch definiert, nicht reproduziert werden: weder von Tönges et al. (2014) bei einem präsymptomatischen Therapiebeginn am Lebenstag 50, noch von Günther (2015) bei einem symptomatischen Therapiebeginn am Lebenstag 80. Auch in der vorliegenden Studie bei einem präsymptomatischen Behandlungsbeginn am Lebenstag 50 kann in keiner Behandlungsgruppe ein verzögerter Krankheitsbeginn im Vergleich zur unbehandelten Kontrollgruppe beobachtet werden.

Es kann jedoch ein signifikant früheres Auftreten erster Symptome in der Fas10-Gruppe beobachtet werden. Im Durchschnitt zeigen diese Tiere schon mit fast 90 Tagen erste Symptome (Tremor der Hinterläufe), während unbehandelte Tiere im Durchschnitt diese erst am Lebenstag 103 zeigen. Tiere aus der Ril30-Gruppe, aus der Fas30-Gruppe und aus der Fas10Ril30-Gruppe zeigen einen zur unbehandelten Veh-Gruppe nicht signifikant früheren oder späteren Symptombeginn. Auf das Gesamtüberleben hat dieser frühere Krankheitsbeginn in der Fas10-Gruppe keinen Einfluss, sie haben daher eine signifikant längere Krankheitsdauer als die Veh-Kontrollgruppe. Auch den Score 2 und den Score 1 erreichen alle Gruppen im Mittel zu Zeitpunkten, die sich nicht signifikant voneinander unterscheiden. Für die Behandlung mit Fas30 zeigen sich zwar hier starke Trends mit erst später erreichten Scores 2 und 1, die jedoch nicht das Signifikanzniveau erreichen. Somit kann in der vorliegenden Studie kein Unterschied im Zeitpunkt des Erreichens der Scores 2 oder 1 zwischen den behandelten Gruppen und der unbehandelten Kontrollgruppe festgestellt werden. Dies bestätigt die Ergebnisse von Günther (2015), der in bei einem symptomatischen Behandlungsbeginn mit Fasudil (30 mg/kg KG und 100 mg/kg KG) am Lebenstag 80 ebenfalls keine Unterschiede im Verlauf der Symptome feststellen kann.

Zusammenfassen lassen sich die Erkenntnisse wie folgt:

1. Den Krankheitsbeginn können Riluzol (30 mg/kg KG), Fasudil (30 mg/kg KG), Fasudil (10 mg/kg KG) und die Kombinationstherapie aus Fasudil (10 mg/kg KG) mit Riluzol (30 mg/kg KG) nicht verzögern und den Krankheitsverlauf, der anhand des klinisch-neurologischen Scores beurteilt wurde, nicht abmildern. Dies entspricht den Ergebnissen von Tönges et al. (2014) und Günther (2015). Dagegen können Takata et al. (2013) einen verzögerten Krankheitsbeginn durch die Behandlung mit Fasudil (30 mg/kg KG und $100 \mathrm{mg} / \mathrm{kg} \mathrm{KG}$ ) bei einem Behandlungsbeginn am Lebenstag 35 feststellen. Eine längere Applikationsdauer 
durch einen frühen Behandlungsbeginn scheint einen maßgeblichen Einfluss auf den Krankheitsverlauf zu haben.

2. Fasudil in einer niedrigen Dosis von $10 \mathrm{mg} / \mathrm{kg} \mathrm{KG}$ ergibt per Definition einen früheren Symptombeginn, jedoch nicht einen weiteren schlechteren klinischen Verlauf, einen früheren Gewichtsverlust oder eine schlechtere motorische Entwicklung.

\subsubsection{Fasudil verbessert die motorische Performance}

Ziel der vorliegenden Studie ist es, im Rahmen einer Verlaufsstudie die motorische Entwicklung in den verschiedenen Behandlungsgruppen $\mathrm{zu}$ beobachten. Als Kontrollgruppen dienen: eine unbehandelte Gruppe transgener Mäuse und eine gesunde Kontrollgruppe mit Wildtyp-Tieren. Es wird einerseits zweimal wöchentlich die Motorkoordination mittels Drehwalzenversuch (Rotarod) getestet und andererseits einmal wöchentlich eine videobasierte Ganganalyse (CatWalk XT) durchgeführt.

In der vorliegenden Studie können keine signifikant lebensverlängernden, den Krankheitsverlauf verlangsamenden Einflüsse oder günstige Effekte auf das Überleben der Alpha-Motoneurone im lumbalen Rückenmark durch Fasudil, Riluzol oder die Kombinationsbehandlung festgestellt werden. Es können jedoch günstige Effekte auf die makroskopisch untersuchte Muskelatrophie durch die Therapie mit Riluzol $(30 \mathrm{mg} / \mathrm{kg}$ $\mathrm{KG})$, Fasudil in niedriger $(10 \mathrm{mg} / \mathrm{kg} \mathrm{KG})$ und Fasudil in mittlerer Dosierung $(30 \mathrm{mg} / \mathrm{kg}$ KG) gezeigt werden: Der Anteil des Musculus gastrocnemius am Körpergewicht befindet sich am Untersuchungszeitpunkt, Lebenstag 120, unter der Behandlung mit Riluzol und unter der Behandlung mit Fasudil (10mg/kg KG) auf dem Niveau von gesunden Tieren, während bei den unbehandelten Tieren ein Verlust von fast $40 \%$ zu beobachten ist (siehe Kapitel 4.2.1).

Diese Befunde werden von den Ergebnissen der motorischen Testungen der Verlaufsstudie unterstützt. Hier zeigen sich positive Effekte auf die Motorkoordination im Drehwalzenversuch (Rotarod) durch die Therapie mit Fasudil in mittlerer Dosierung (30 $\mathrm{mg} / \mathrm{kg} \mathrm{KG)}$. In der videobasierten Ganganalyse können günstige Effekte in mehreren Behandlungsgruppen gezeigt werden, insbesondere an einem späten Versuchstag, Lebenstag 127. Auch hier sticht die Behandlung mit Fasudil in mittlerer Dosierung mit besonders guten Ergebnissen hervor.

4.1.3.1 Fasudil (30 mg/kg KG) verbessert milde die Leistung im Drehwalzentest (Rotarod) Ziel der Verlaufsstudie ist es mittels Drehwalzenversuch (Rotarod) die motorkoordinativen Fähigkeiten der Mäuse unter der Behandlung zu beobachten und mögliche günstige 
Effekte durch die verschiedenen Behandlungen auf die motorische Entwicklung der Tiere zu beobachten.

Takata et al. (2013) können durch die Behandlung mit Fasudil (30 mg/kg KG und 100 $\mathrm{mg} / \mathrm{kg} \mathrm{KG}$ ) beginnend am Lebenstag 35 eine Verzögerung bei der Entwicklung von Defiziten in der Motorkoordination zeigen. Dies gelingt auch Tönges et al. (2014) durch die Behandlung mit Fasudil beginnend am Lebenstag 50 in der Dosierung $30 \mathrm{mg} / \mathrm{kg} \mathrm{KG}$ (Suhr 2017). Und Günther (2015) kann selbst durch eine symptomatische Behandlung beginnend am Lebenstag $80 \mathrm{mit}$ Fasudil (30 mg/kg KG und $100 \mathrm{mg} / \mathrm{kg} \mathrm{KG}$ ) bei männlichen Tieren eine Verbesserung der motorkoordinativen Fähigkeiten im Drehwalzenversuch beobachten (Günther et al. 2017).

Im Rahmen der vorliegenden Studie ergibt sich im Drehwalzenversuch (Rotarod) über den gesamten Untersuchungszeitraum kein signifikanter Unterschied zwischen der unbehandelten Veh-Kontrollgruppe und den Behandlungsgruppen. Signifikant ist der Unterschied nur zu den gesunden Wildtyp-Tieren (siehe Abbildung 19). Im Gegensatz zu allen anderen Gruppen unterscheidet sich die Gruppe der Tiere, die mit Fasudil in mittlerer Dosierung behandelt wurden $(30 \mathrm{mg} / \mathrm{kg} \mathrm{KG})$ nicht signifikant von den gesunden Tieren. So lassen sich zusammenfassend milde bessernde Effekte durch die Behandlung mit Fasudil (30 mg/kg KG) beobachten, die jedoch in ihrer Signifikanz hinter denen von Takata et al. (2013) und Tönges et al. (2014) zurückbleiben. Eine Therapie mit Fasudil in niedriger Dosierung (10 mg/kg KG) scheint diese Wirkung nicht mehr entfalten zu können. Ebenso kann eine Monotherapie mit Riluzol oder die Kombinationsbehandlung mit beiden Präparaten, wobei Fasudil niedrig dosiert (10 mg/kg KG) wurde, keine günstigen Effekte auf die Motorkoordination im Drehwalzenversuch zeigen.

Zusammenfassen lassen sich die Erkenntnisse wie folgt:

1. Die Behandlung mit Fasudil (30 mg/kg KG) kann in der vorliegenden Studie milde vorteilhafte Effekte auf die Motorkoordination zeigen: Über den gesamten Untersuchungszeitraum (Lebenstag 50 bis 123) war die Gruppe nicht signifikant schlechter als gesunde Wildtyptiere, im Gegensatz zur unbehandelten VehKontrollgruppe und allen anderen Behandlungsgruppen.

2. Die Behandlung mit Fasudil in niedriger Dosierung $(10 \mathrm{mg} / \mathrm{kg} \mathrm{KG})$, die Behandlung mit Riluzol (30 mg/kg KG) und eine Kombination beider Behandlungen können keine protektiven Effekte auf die Motorkoordination zeigen. 


\subsubsection{Protektive Effekte durch Fasudil und Riluzol in videobasierter Ganganalyse (CatWalk XT)}

Ziel der Verlaufsstudie ist es mit der videobasierten Ganganalyse bei sich frei bewegenden Mäusen (CatWalk XT) die motorischen Fähigkeiten unter der Behandlung detailliert zu beobachten und mögliche günstige Effekte durch die verschiedenen Behandlungen auf die motorische Entwicklung der Tiere noch genauer zu beobachten.

Hauptparameter ist dabei die Durchschnittsgeschwindigkeit (mean average speed). Daneben werden die Defizite im Gebrauch der Hinterläufe mithilfe der folgenden Parameter des rechten Hinterlaufs analysiert: Auftrittsfläche (print area), Schrittgeschwindigkeit (swing speed), Auftrittslänge (print length) und Schrittlänge (stride length). Zum Vergleich werden diese Parameter auch für den rechten Vorderlauf ausgewertet.

\subsection{Erhöhte Leistungsfähigkeit durch Fas30 in der letzten Krankheitsphase}

In der vorliegenden Studie wird die Durchschnittsgeschwindigkeit als ein Parameter für die allgemeine motorische Leistungsfähigkeit ausgewertet. Es zeigt sich eine konstante Entwicklung in allen Gruppen bis einschließlich dem Lebenstag 106. Dann insbesondere an den letzten beiden Untersuchungstagen 120 und 127 eine signifikante Verschlechterung der transgenen Tiere gegenüber den gesunden Tieren. Am Lebenstag 127 kann allerdings eine signifikant schnellere Durchschnittsgeschwindigkeit der mit Fasudil (30 mg/kg KG) behandelten Tiere gegenüber der unbehandelten Veh-Kontrollgruppe beobachtet werden. Dies spricht für eine verbesserte allgemeine motorische Leistungsfähigkeit der Tiere in dieser späten Krankheitsphase.

\subsection{Verminderte Paralyse der Hinterläufe durch Fas30}

Die Hinterläufe sind im Krankheitsverlauf des verwendeten Mausmodells einer besonders rapiden Lähmungsentwicklung unterworfen, und können im Zuge dessen immer weniger zur Fortbewegung eingesetzt werden. Dies zeichnet sich zunächst dadurch aus, dass die Tiere den stärker betroffenen Lauf auch weniger stark benutzen und dementsprechend mit ihm weniger auftreten. Zudem können Defizite beim Abrollen beobachtet werden. Die Tiere treten mit einem betroffenen Hinterlauf nicht mehr mit dem Fußballen auf, sondern nur noch mit den Zehen. In der letzten Krankheitsphase ist diese Entwicklung letztendlich durch das Hinterherschleifen eines Laufs oder sogar beider Hinterläufe gekennzeichnet. Diese funktionellen Überlegungen spielen für die Auftrittsfläche und die Auftrittslänge eine entscheidende Bedeutung. Sie sind bei der Interpretation der Ergebnisse der Hinter- und Vorderläufe zu beachten. 
Über den gesamten Untersuchungszeitraum lässt sich kein Unterschied zwischen gesunden Wildtyp-Tieren und transgenen Tieren feststellen. Am letzten Untersuchungstag aber, dem Lebenstag 127, zeigt sich eine signifikant niedrigere Auftrittsfläche bei transgenen, unbehandelten Tieren. Auch die Tiere der Fas10-Gruppe und der Fas10Ril30-Gruppe haben eine signifikant niedrigere Auftrittsfläche als gesunde Wildtyp-Tiere, im Gegensatz zu den Tieren, die mit Riluzol (30 mg/kg KG) und denen, die mit Fasudil (30 mg/kg KG) behandelt wurde. Die Fas30-Gruppe hat zudem eine signifikant höhere Auftrittsfläche als unbehandelte transgene Tiere. Das spricht für einen weitgehenden Erhalt der motorischen Funktion des Hinterlaufs und eine bessere Einsetzbarkeit beim Laufen durch die Therapie mit Fasudil (30 mg/kg KG) am Ende der Erkrankung.

\subsection{Fasudil, Riluzol und Kombination bremsen motorische Kompensations- mechanismen}

Durch die zunehmende Lähmung der Hinterläufe sind die Mäuse gezwungen vermehrt die Vorderläufe $\mathrm{zu}$ beanspruchen, um sich effektiv fortzubewegen. Es findet ein immer stärkeres Hinterherschleifen der Hinterläufe statt, was eine stärkere Beanspruchung der Vorderläufe zur Folge hat.

Es kann in der Untersuchung über den gesamten Untersuchungszeitraum kein signifikanter Gruppenunterschied zwischen gesunden und kranken Tieren festgestellt werden, aber in der Analyse des Untersuchungszeitraums von Lebenstag 106 bis 127 mit einer ANOVA mit Messwiederholung lässt sich eine signifikant höhere Auftrittsfläche der Vorderläufe bei unbehandelten erkrankten Tiere im Vergleich zu den gesunden Wildtyp-Tieren feststellen. Alle behandelten Gruppen zeigen diese signifikante Erhöhung der Auftrittsfläche des Vorderlaufs nicht, aber unterschieden sich auch nicht signifikant von der unbehandelten Kontrollgruppe. So lässt sich schließen, dass die unbehandelten erkrankten Tiere deutlich stärker auf die Funktion der Vorderläufe zurückgreifen müssen, um den Funktionsverlust der Hinterläufe zu kompensieren. Eine Behandlung mit Fasudil in beiden erprobten Dosierungen, Riluzol und eine Kombination kann die Entwicklung zumindest milde aufhalten. Am letzten Untersuchungszeitpunkt, dem Lebenstag 127, kann die Behandlung mit Riluzol die Überbeanspruchung des Vorderlaufs als Kompensation des gelähmten Hinterlaufs signifikant gegenüber der unbehandelten Kontrollgruppe senken.

\subsection{Fasudil (30 mg/kg KG) erhält den funktionellen Gebrauch des Hinterlaufs}

Im Rahmen der Lähmungsentwicklung einer Extremität geht nicht nur deren Gesamtbeanspruchung zurück, was sich im Rückgang der Auftrittsfläche zeigt, auch die funktionelle Benutzung im Schrittablauf, das normale Abrollen, die Beanspruchung des 
Fußballens geht immer mehr verloren. Dies zeigt sich vor allem im Rückgang der Auftrittslänge eines Laufs. Über den gesamten Untersuchungszeitraum konnte sich kein signifikanter Gruppenunterschied zeigen. Aber in der Analyse mit einer ANOVA mit Messwiederholung der letzten fünf Untersuchungszeitpunkte (Lebenstag 99 bis 127) zeigt sich eine signifikant kürzere Auftrittslänge der unbehandelten Kontrollgruppe gegenüber den gesunden Wildtyp-Tieren. Die Behandlung mit Fasudil (30 mg/kg KG) kann diese Entwicklung vollständig aufhalten. In diesem Zeitraum ist die Auftrittslänge signifikant länger als die der unbehandelten Veh-Kontrollgruppe und zeigt keinen Unterschied zu den gesunden Wildtyp-Tieren, während die übrigen Behandlungsgruppen eine solche signifikante Besserung nicht erzielen, aber deren Ergebnisse auch nicht signifikant schlechter sind als die der gesunden Wildtyp-Tiere. Dieselben Ergebnisse ergeben sich bei der Auswertung des letzten Untersuchungszeitpunkts, Lebenstag 127. So lässt sich schließen, dass Fasudil (30mg/kg KG) die funktionelle Benutzung der Hinterläufe bis in die späte Krankheitsphase erhält, während alle anderen Behandlungen nur einen milden protektiven Effekt haben. Für den Vorderlauf lassen sich über den gesamten Untersuchungszeitraum keine Unterschiede in der funktionellen Benutzung im Sinne einer veränderten Auftrittslänge feststellen.

\subsection{Fasudil (30 mg/kg KG) verbessert die Beweglichkeit des Hinterlaufs in der} letzten Krankheitsphase

Neben der Funktionalität spielt bei der Lähmung vor allem die Beweglichkeit und Kraft einer Extremität eine tragende Bedeutung. Für die Messung dieser motorischen Funktionen sind die Schrittlänge und die Schrittgeschwindigkeit, also die Schnelligkeit, mit der die Extremität bewegt wird, für die Analyse herangezogene Parameter.

Die Schrittgeschwindigkeit zeigt über den gesamten Untersuchungszeitraum keinen signifikanten Unterschied zwischen gesunden und unbehandelten kranken Tieren. Die mit Fasudil (30 $\mathrm{mg} / \mathrm{kg} \quad \mathrm{KG})$ zeigen hier insgesamt eine signifikant langsamere Schrittgeschwindigkeit. In der Auswertung der letzten fünf Untersuchungszeitpunkte mit einer ANOVA mit Messwiederholung zeigt sich jedoch eine signifikant langsamere Schrittgeschwindigkeit der unbehandelten kranken Tiere und der Tiere der Kombinationsbehandlung gegenüber den gesunden Wildtyp-Tieren. Die anderen Behandlungsgruppen unterscheiden sich zwar nicht signifikant von den Wildtyp-Tieren, können aber in diesem Zeitraum keine signifikant schnellere Schrittgeschwindigkeit aufweisen als die unbehandelte Kontrollgruppe. Einzig am letzten Untersuchungszeitunkt, Lebenstag 127, kann die Behandlung mit Fasudil (30 mg/kg KG) eine signifikant höhere 
Schrittgeschwindigkeit zur unbehandelten Kontrolle erzielen, verbleibt aber wie alle anderen signifikant langsamer als die der gesunden Wildtyp-Tiere.

Die Schrittgeschwindigkeit des Vorderlaufs unterscheidet sich über den gesamten Untersuchungszeitraum nicht signifikant zwischen den Gruppen, einzig die Fas30-Gruppe hat eine signifikant langsamere Schrittgeschwindigkeit als die Wildtyp-Tiere. Eine Verlangsamung der Schrittgeschwindigkeit der Vorderläufe bei den transgenen Tieren findet etwas später statt als bei den Hinterläufen: In der ANOVA mit Messwiederholung der letzten vier Untersuchungszeitpunkte ist die Schrittgeschwindigkeit in allen Gruppen transgener Tiere langsamer als die der gesunden Wildtyp-Tiere und von der Behandlung kann keine der Gruppen profitieren, ebenso nicht am letzten untersuchten Lebenstag 127.

\subsection{Fasudil und Kombination erhalten die Kraft in den Extremitäten}

Die Schrittlänge kann als ein Parameter für die Kraft einer Extremität im Schrittablauf gelten und die des Hinterlaufs der unbehandelten transgenen Tiere ist gesamten Untersuchungszeitraum signifikant kürzer als die der Wildtyp-Tiere, ebenso wie die der Fas30-Gruppe und die der Ril30-Gruppe. Nicht signifikant kürzer als die gesunde Kontrollgruppe sind die Werte der Fas10-Gruppe und die der Fas10Ril30-Gruppe. Es zeigt sich allerdings kein Behandlungseffekt im Vergleich zu unbehandelten Kontrollgruppe bei den transgenen Tieren. Lediglich am letzten Untersuchungszeitpunkt, Lebenstag 127, erzielt die Behandlung mit Fasudil (30 mg/kg KG) und die Behandlung mit der Kombination aus Fasudil (10 mg/kg KG) und Riluzol (30 mg/kg KG) eine bessernde Wirkung: Die Schrittlänge ist signifikant länger als die rapide gesunkene der unbehandelten Kontrolle und unterscheidet sich nicht signifikant von den gesunden Tieren.

So lässt sich schließen, dass Fasudil in niedriger Dosierung auch in Kombination mit Riluzol insgesamt eine milde Verbesserung der Schrittlänge über den gesamten Krankheitsverlauf bewirken kann, in der letzten Krankheitsphase aber eine signifikante bessernde Wirkung auf die Schrittlänge nur in Kombination mit Riluzol oder in der höheren Dosierung (30 mg/kg KG) entfalten kann. Ein fast gleiches Bild lässt sich für die Schrittlänge des Vorderlaufs zeichnen, wobei hier in der letzten Krankheitsphase (Lebenstag 127) nur die Behandlung mit Fasudil (30 mg/kg KG) eine signifikante Verlängerung der Schrittlänge bewirkt.

\subsection{Fazit zur videobasierten Ganganalyse}

Zusammenfassen lassen sich die Erkenntnisse wie folgt:

1. Die videobasierte Ganganalyse mittels CatWalk XT ist eine gute Methode, um die motorischen Defizite im SOD1G93A-Mausmodell vor allem in der letzten 
Krankheitsphase detailliert zu beurteilen: Die Durchschnittsgeschwindigkeit (mean average speed) kann als $\mathrm{Maß}$ für die allgemeine motorische Leistungsfähigkeit, die Auftrittsfläche (print area) als Maß für die Paralyse einer Extremität, die Auftrittslänge (print length) für den funktionellen Gebrauch einer Extremität und die Schrittgeschwindigkeit (swing speed) und Schrittlänge (stride length) als Maß für die Beweglichkeit bzw. Kraft einer Extremität verwendet werden.

2. Die Behandlung mit Fasudil (30 mg/kg KG) verringert die Paralyse der Hinterläufe und erhöht die allgemeine motorische Leistungsfähigkeit, den funktionellen Gebrauch der Hinterläufe und die Beweglichkeit und Kraft der Hinterläufe vor allem in der letzten Krankheitsphase.

3. Die erkrankten Tiere verwenden bei verstärkter Paralyse der Hinterläufe kompensatorisch mehr die Vorderläufe, was durch eine Behandlung mit Riluzol (30 $\mathrm{mg} / \mathrm{kg} \mathrm{KG)} \mathrm{abgemildert} \mathrm{werden} \mathrm{kann.}$

4. Fasudil $(10 \mathrm{mg} / \mathrm{kg} \mathrm{KG})$ verbessert auch in Kombination mit Riluzol $(30 \mathrm{mg} / \mathrm{kg}$ KG) über den gesamten Krankheitsverlauf die Kraft und Beweglichkeit der Vorder- und Hinterläufe.

\subsubsection{SOD1G93A-Mausmodell und kognitive Verhaltensbiologie}

Die Durchführung des novel object recognition (NOR)-Tests dient der Beobachtung der kognitiven Performance im Krankheitsverlauf. In den letzten Jahren sind neben der Beobachtung von kognitiven Defiziten bei ALS-Patienten auch Gemeinsamkeiten der ALS mit der FTLD auf der Ebene der Pathomechanismen beider Erkrankungen gefunden worden (Brettschneider et al. 2013). So soll dieser Test, der an vier festgelegten Tagen durchgeführt wird (Tag 50, 88, 102 und 116), die Frage nach einem Verhaltensunterschied der kranken Tiere zu gesunden klären und einen möglichen Einfluss der Behandlung auf die kognitive Entwicklung klären.

In der vorliegenden Studie kann kein wesentlicher Unterschied in der zurückgelegten Strecke (Abbildung 37) und kein Unterschied im DI (Abbildung 38) gefunden werden. Lediglich in der zurückgelegten Strecke ergeben sich Gruppenunterschiede an Tag 102 und Tag 116: An Tag 102 legen die Tiere der Ril30-Gruppe signifikant mehr Strecke zurück als die Tiere der Veh-Gruppe, sodass entweder eine bessere motorische Performance geleistet werden kann oder ein erhöhtes Explorationsverhalten vorliegt. Dies spiegelt sich jedoch nicht in einer erhöhten Diskriminationsfähigkeit der Tiere wider. Daneben kann eine signifikant niedrigere zurückgelegte Strecke der Tiere der Fas10Ril30-Gruppe im Vergleich zur denen der Fas30-Gruppe gefunden werden. Im Vergleich zur gesunden Wt- 
Kontrollgruppe oder zur unbehandelten Veh-Kontrollgruppe können diese Unterschiede allerdings nicht gefunden werden. Die Unterschiede zwischen Wildtyp-Tieren und transgenen Tieren, die Spalloni et al. (2006) im Explorationsverhalten gefunden haben, können nicht reproduziert werden.

Zusammenfassen lassen sich die Erkenntnisse wie folgt:

1. Explorationsverhalten und Diskriminationsfähigkeit unterscheiden sich zwischen gesunden und kranken Tieren im SOD1G93A-Mausmodell im Rahmen des NORTests nicht.

2. Die Behandlung mit Fasudil, Riluzol oder einer Kombination hat keinen wesentlichen negativen, noch einen wesentlichen positiven Effekt auf Explorationsverhalten oder Diskriminationsfähigkeit.

3. Zur Untersuchung der Effekte von Fasudil und Riluzol auf kognitive Parameter mittels NOR-Test sollten in der Zukunft andere Modelle für die ALS als das SOD1G93A-Mausmodell, das keinen Unterschied zwischen gesunden und kranken Tieren aufweist, genutzt werden.

\subsection{Querschnittstudie nach präsymptomatischer Behandlung mit Fasudil und Riluzol}

Um einen Behandlungseffekt auf histologischer Ebene observieren zu können, wird unter Berücksichtigung vorangehender Studien der Lebenstag 120 als Analysezeitpunkt festgesetzt: Zwar wird die Degeneration der Alpha-Motoneurone im Lumbalmark in diesem Mausmodell schon zu einem früheren Analysezeitpunkt, am Lebenstag 100, beobachtet (Fischer et al. 2004, Takata et al. 2013), aber im Rahmen unserer Arbeitsgruppe können wir bei Untersuchungen zu diesem Zeitpunkt noch keine signifikant niedrigere Anzahl von Alpha-Motoneuronen im lumbalen Rückenmark bei SOD1G93A transgenen Mäusen im Vergleich zum Wildtyp-Tier beobachten (Günther 2015). Daher wird ein späterer Zeitpunkt für die Querschnittstudie gewählt.

Als Untersuchungsparameter werden im Rahmen dieser Studie die Anzahl von AlphaMotoneuronen pro Vorderhorn und der prozentuale Anteil des Musculus gastrocnemius am Körpergewicht am Lebenstag 120 festgelegt. Dafür wird das Körpergewicht vor der Euthanasie der Tiere gemessen. Nach Euthanasie, Perfusion mit PBS und 4\%igem PFA des Tieres wird der Musculus gastrocnemius präpariert und mit einer Feinwaage dessen Gewicht gemessen. Anschließend wird das Rückenmark präpariert, nachfixiert und dehydriert. Auf Kryo-Schnitten des lumbalen Rückenmarks werden die AlphaMotoneurone immunhistochemisch angefärbt und gezählt. 


\subsubsection{Protektive Effekte auf die Muskelatrophie durch Fasudil und Riluzol}

Nach Euthanasie am Lebenstag 120 wird der prozentuale Anteil des Musculus gastrocnemius am Körpergewicht errechnet. Während dieser beim gesunden Tier am Lebenstag 120 im Mittel bei 0,57\% liegt, ist der Anteil beim unbehandelten transgenen Tier zum selben Zeitpunkt auf 0,36\% zurückgegangen. Wenn dieser Muskelanteil von 0,57\% als Normwert vorausgesetzt wird, bedeutet dies für die erkrankten Tiere einen Verlust von 36,84\% der Muskelmasse dieses Muskels. Mohajeri et al. (1998) können im Alter von 18 Wochen - also ca. eine Woche später - einen Gewichtsverlust des Musculus gastrocnemius von 45\% bei SOD1G93A-transgenen Mäusen im Vergleich zu Wildtyp-Tieren feststellen. Die Beobachtungen zum Ausmaß der Muskelatrophie im Rahmen der vorliegenden Studie decken sich in etwa mit denen von Mohajeri et al. (1998). Günther (2015) kann schon am Lebenstag 100 - fast drei Wochen zuvor - einen signifikant geringeren Anteil des Musculus gastrocnemius am Körpergewicht bei transgenen Tieren im Vergleich zum Wildtyp-Tier beobachten. Zu diesem frühen Zeitpunkt beobachtet er einen Verlust von $12 \%$ des Muskelanteils im Vergleich zum gesunden Tier. Durch die Behandlung mit $30 \mathrm{mg} / \mathrm{kg} \mathrm{KG}$ Fasudil kann dabei zu diesem Zeitpunkt noch kein Einfluss auf die Muskelatrophie beobachtet werden.

In der vorliegenden Studie kann durch die Behandlung mit Fasudil (10 mg/kg KG) oder mit Riluzol (30 mg/kg KG) der Verlust an Muskelmasse vollständig aufgehalten werden. Auch die Behandlung mit Fasudil (30 mg/kg KG) hat im Vergleich zu erkrankten Tieren einen protektiven Effekt. Im Vergleich zum gesunden Tier ist der Muskelanteil der Tiere aus der Ril30-Gruppe und aus der Fas10-Gruppe nicht signifikant gemindert, im Vergleich zu den unbehandelten Tieren ist er signifikant erhöht (siehe Abbildung 36). Die Tiere aus der Fas30-Gruppe haben einen signifikant höheren Muskelanteil als unbehandelte Tiere, aber einen geminderten im Vergleich zur gesunden Kontrollgruppe.

Bei den Tieren, die mit der Kombination Fasudil und Riluzol behandelt werden, kann kein vorteilhafter Effekt beobachtet werden. Im Hinblick auf die Muskelatrophie zeigt die RhoKinase-Inhibition mit Fasudil in niedriger Dosierung und die gleichzeitige antiglutamaterge Therapie mit Riluzol keine Synergieeffekte. Im Gegenteil: Die bei der jeweiligen Monotherapie beobachteten günstigen Effekte werden durch eine gemeinsame Therapie wieder aufgehoben.

An dieser Stelle lohnt ein genauer Blick auf die Wirkmechanismen beider Medikamente und mögliche Schnittstellen, um die Hintergründe dieser Beobachtung zu beleuchten: Eine frühe pathogenetische Beobachtung bei der ALS ist der selektive Verlust des glutmate transporter 1 (GLT1) im Motorkortex und Rückenmark von ALS-Patienten, der dem 
excitatory amino acid transporter 2 (EAAT2) entspricht (Rothstein und Kuncl 1995, siehe Kapitel 1.1.5, Abbildung 2). Dieser Natrium-abhängige Transporter sorgt für eine Wiederaufnahme von Glutamat aus dem synaptischen Spalt in Astrozyten und ist einer der zentralen Transporter zur Gewährleistung einer Glutamat-Clearance im synaptischen Spalt: Sein Fehlen führt zu erhöhten extrazellulären Glutamat-Spiegeln in vitro, die eine für Exzitotoxizität charakteristische Neurodegeneration - wie bspw. geschwollene Dendriten und progressive Lähmungen in vivo zur Folge haben (Rothstein 1996). Exzessiv erhöhte Glutamat-Spiegel im synaptischen Spalt können zu einem neurotoxisch wirksamen Einstrom von Calcium-Ionen führen.

In ALS-Modellen kann zudem eine erhöhte Caspase 3-Aktivität demonstriert werden (Pasinelli et al. 2000). Eine proteolytische Spaltung am zytosolischen C-Terminus von EAAT2 durch Caspase 3 und eine damit einhergehende Inhibition scheint für den Funktionsverlust des Transporters ursächlich zu sein. Durch die Behandlung mit rekombinanter humaner Caspase 3 von murinen Rückenmarkslysaten verliert der Transporter dosisabhängig seine Immunoreaktivität im Western Blot, während eine niedrigere Bande, die dem N-Terminus von EAAT2 entspricht, entsprechend vermehrt nachweisbar wird (Boston-Howes et al. 2006).

Riluzol erhöht die Glutamat-Wiederaufnahme dosisabhängig durch eine unselektive Aktivierung von Glutamat-Transportern, darunter auch EAAT2. Die molekularen Wirkmechanismen von Riluzol auf EAAT2 sind nicht geklärt. Eine Hypothese ist, dass eher die Glutamat-Affinität des Transporters als seine Expression erhöht wird (Fumagalli et al. 2008, Foran und Trotti 2009).

Die Aktivierung von ROCK basiert auf der Unterbrechung der Kinase-Einheit von regulatorischen Elementen im C-Terminus, was durch die Bindung von RhoA verursacht wird, aber auch durch die Abspaltung des C-Terminus durch Caspase 3 verursacht werden kann (Jacobs et al. 2006, Coleman et al. 2001, siehe Kapitel 1.3.1). So führt eine erhöhte Caspase 3-Aktivität bei ALS neben einer Inaktivierung von EAAT2 zu einer erhöhten ROCK-Aktivität und verursacht so auf beiden Wegen eine fortschreitende Neurodegeneration. Es kann somit geschlussfolgert werden, dass Fasudil und Riluzol Mechanismen blockieren, die durch Caspase 3 mit ausgelöst werden.

Darüberhinaus kann gezeigt werden, dass durch eine Fasudil-Behandlung die Expression von EAAT2 in Astrozyten erhöht wird, sowohl im Zytosol als auch an der Zelloberfläche (Lau et al. 2011). So kann durch Fasudil die Expression und durch Riluzol die Affinität des Transporters erhöht werden. Beide Mechanismen sollten so letzendlich zu einer verbesserten Glutamat-Clearance führen. 
In Anbetracht dieser grundsätzlichen Überlegungen auf molekularer Ebene liegt der Schluss nahe, dass sich Fasudil und Riluzol in ihrer Wirksamkeit prinzipiell ergänzen können. Eine gemeinsame Verabreichung erscheint vor diesem Hintergrund sinnvoll, und additive oder sogar synergistische Effekte sind denkbar.

Die Ergebnisse der vorliegenden Querschnittstudie unterstützen die These der synergistischen Wirkung nicht. Im Gegenteil: Vorteilhafte Effekte beider Medikamente gehen bei gemeinsamer Verabreichung verloren.

In diesem Modell könnten bisher unbekannte molekulare Wirkmechanismen beider Medikamente als Ursache dieser Beobachtung in Frage kommen. Dabei könnte Caspase 3 als Modulator der Effekte beider Medikamente eine entscheidende Rolle spielen. Denkbar sind Steuerungsmechanismen, die bei gemeinsamer Verabreichung einen stimulierenden Einfluss auf die Caspase 3-Aktivität haben und so die günstigen Effekte aufheben.

Dabei muss als Ursache der schädlichen Wechselwirkung auch die Dosierung beleuchtet werden: So könnte Fasudil in einer höheren Dosierung in Kombination mit Riluzol die dargelegten Synergiemechanismen möglicherweise wirksamer nutzen. Denn in den Ergebnissen der vorliegenden Verlaufsstudie schneidet Fasudil $30 \mathrm{mg} / \mathrm{kg}$ KG durchweg besser ab als in niedriger Dosierung. Eine genau ausbalancierte Dosierung beider Präparate erscheint sinnvoll. Daher sollten weitere In-vivo-Untersuchungen mit höher dosiertem Fasudil in Kombination mit Riluzol erfolgen. Die gemeinsamen molekularen Synergiemechanismen beider Präparate sind noch immer als vielversprechend zu werten. Eine In-vitro-Untersuchung der Expression von EAAT2 und des extrazellulären GlutamatSpiegels unter der Kombinationsbehandlung mit Fasudil und Riluzol sind sinnvolle Untersuchungen für die Zukunft, um die Frage nach möglicherweise dosisabhängigen Wechselwirkungen zu klären. Dabei sollte auch der Einfluss auf diese Parameter durch Caspase 3 berücksichtigt werden.

Zusammenfassen lassen sich die Erkenntnisse wie folgt:

1. Der Lebenstag 120 ist ein guter Analysezeitpunkt für Querschnittstudien, die die Muskelatrophie bei Tieren des transgenen SOD1G93A-Mausmodells makroskopisch untersuchen: Es lässt sich ein signifikant niedrigerer Anteil des Musculus gastrocnemius am Körpergewicht im Vergleich zu gesunden WildtypTieren zeigen.

2. Fasudil (10 mg/kg KG) und Riluzol (30 mg/kg KG) halten den Muskelanteil am Körpergewicht am Lebenstag 120 auf dem Niveau von gesunden Wildtyp-Tieren, welcher signifikant höher ist als der von transgenen unbehandelten Tieren. 
3. Fasudil (30 mg/kg KG) hat einen protektiven Effekt auf die Muskelatrophie, hält den Muskelanteil jedoch nicht auf dem Niveau von gesunden Tieren.

4. Durch eine Kombination von Fasudil in der niedrigen Dosierung (10 mg/kg KG) mit Riluzol (30 mg/kg KG) verliert sich der protektive Effekt beider Substanzen trotz denkbarer molekularer Synergiemechanismen. Eine Untersuchung der Kombinationsbehandlung mit einer höheren Dosierung von Fasudil ist eine sinnvolle Untersuchung für die Zukunft. Zudem sollte ein möglicherweise dosisabhängiger Effekt einer Kombinationsbehandlung auf die Glutamat-Clearance im synaptischen Spalt und die Expression von EAAT2 in vitro untersucht werden.

\subsubsection{Unbeeinflusster Verlust der Alpha-Motoneurone im lumbalen Rückenmark}

Die Anzahl der Alpha-Motoneurone pro Vorderhorn am Lebenstag 120 wird mithilfe der immunhistochemischen ChAT-Färbung an Gefrierschnitten des lumbalen Rückenmarks untersucht. Sie soll den Einfluss von Fasudil in niedriger $(10 \mathrm{mg} / \mathrm{kg} \mathrm{KG})$ und mittlerer (30 mg/kg KG) Dosierung und Riluzol sowie einer Kombinationsbehandlung auf die Degeneration der Alpha-Motoneurone untersuchen.

Bei unbehandelten SOD1G93A-transgenen Tieren der Veh-Kontrollgruppe zeigt sich eine signifikant geringere Anzahl von Alpha-Motoneuronen pro Vorderhorn im Vergleich zu gesunden Tieren aus der Wt-Kontrollgruppe. Es kann ein Verlust von 68\% der Motoneurone im Vergleich zum Wildtyptier bei diesen Tieren festgestellt werden (siehe Abbildung 10). Mögliche protektive Effekte der gewählten Therapieansätze auf die Anzahl der Alpha-Motoneurone sollten sich also im Vergleich zur unbehandelten VehKontrollgruppe gut darstellen lassen.

$\mathrm{Zu}$ einem früheren Analysezeitpunkt, dem Lebenstag 100, haben andere Autoren zuvor einen Verlust von 35\% bis 50\% im Vergleich zum Wildtyp-Tier beschrieben (Fischer et al. 2004, Takata et al. 2013). Die Autoren nutzen zur histologischen Analyse und Quantifizierung der Alpha-Motoneurone allerdings eine weniger spezifische Färbemethode, die Nisslfärbung. Die Beobachtung des noch stärkeren Verlusts von Motoneuronen zu einem späteren Zeitpunkt im Rahmen der vorliegenden Studie, der für eine noch weiter fortgeschrittene Degeneration kennzeichnend ist, decken sich mit den Beobachtungen der Autoren. Im Rahmen unserer Forschungsgruppe wurde die Färbung der ChAT-positiven Motoneuronen im lumbalen Rückenmark in vorangegangenen Studien etabliert und durchgeführt. Hier kann am Lebenstag 100 jedoch kein signifikanter Verlust an AlphaMotoneuronen im Vergleich zu Wildtyp-Tieren gezeigt werden. Die Autoren empfehlen daher für Querschnittstudien, die die Anzahl von Alpha-Motoneuronen im lumbalen 
Rückenmark mittels ChAT-Färbung untersuchen, einen späteren Analysezeitpunkt (Günther 2015, Günther et al. 2017). Daher wird in der vorliegenden Studie der Analysezeitpunkt für die Querschnittstudie auf den Lebenstag 120 festgesetzt. In unserer Studie konnte kein protektiver Effekt einer gewählten Therapie auf den Verlust von AlphaMotoneuronen im lumbalen Vorderhorn beobachtet werden.

Takata et al. (2013) können an ihrem Analysezeitpunkt Lebenstag 100 eine signifikant höhere Anzahl von Alpha-Motoneuronen im lumbalen Vorderhorn bei Tieren, die mit 30 $\mathrm{mg} / \mathrm{kg}$ KG Fasudil behandelt wurden, im Vergleich zur unbehandelten Gruppe beobachten. Allerdings findet nicht nur die Querschnittstudie hierbei an einem früheren Zeitpunkt statt, auch der Therapiebeginn wird früher gewählt, im Lebensalter von 5 Wochen. Der protektive Effekt kann in der vorliegenden Studie in der Fas30-Gruppe am gewählten Analysezeitpunkt, Lebenstag 120, und einem Therapiebeginn am Lebenstag 50 nicht reproduziert werden. So muss davon ausgegangen werden, dass ein früher Therapiebeginn einen starken Einfluss auf den neuroprotektiven Effekt der mitteldosierten Fasudil-Therapie hat. Günther (2015) kann ebenfalls bei einem Therapiebeginn am Lebenstag 50 und dem Analysezeitpunkt Lebenstag 100 bzw. Lebenstag 120 keinen signifikant protektiven Effekt der Therapie mit Fasudil $30 \mathrm{mg} / \mathrm{kg} \mathrm{KG}$ auf die bloße Anzahl der Alpha-Motoneuronen im lumbalen Vorderhorn zeigen (Günther et al. 2017). Günther (2015) kann jedoch in der Auswertung der Motoneuronengröße neuroprotektive Effekte der Fasudil-Therapie nachweisen. Die mit Wasser behandelte Gruppe der SOD1G93Atransgenen Tiere hat eine signifikant niedrigere kumulative Motoneuronfläche als die der Wildtyp-Tiere. Bei den mit Fasudil (30 mg/kg KG) behandelten Tieren kann kein signifikanter Unterschied zu den Wildtyp-Tieren gefunden werden.

In der vorliegenden Studie zeigt Fasudil in der niedrigeren Dosierung (10 mg/kg KG) keinen signifikanten Einfluss auf den Zellverlust im lumbalen Vorderhorn. Riluzol (30 $\mathrm{mg} / \mathrm{kg} \mathrm{KG})$ und die Kombinationsbehandlung aus Fasudil (10 mg/kg KG) haben ebenso keinen protektiven Effekt auf den Zellverlust.

Zusammenfassen lassen sich die Erkenntnisse wie folgt:

1. Lebenstag 120 ist ein guter Analysezeitpunkt für Querschnittstudien, die die Anzahl von Alpha-Motoneuronen im lumbalen Rückenmark mithilfe der ChAT-Färbung bei Tieren des transgenen SOD1G93A-Mausmodells untersuchen: Es lässt sich eine signifikant niedrigere Anzahl von Motoneuronen pro Vorderhorn im Vergleich zu gesunden Wildtyp-Tieren zeigen.

2. Riluzol (30 mg/kg KG), Fasudil (10 mg/kg KG), Fasudil (30 mg/kg KG) und Fasudil (10 mg/kg KG) zusammen mit Riluzol (30 mg/kg KG) können den 
Verlust von Motoneuronen, bewertet durch reine Quantifizierung der Motoneuronzahl, nicht beeinflussen.

\subsubsection{Keine Synergieeffekte durch Kombination mit niedrig dosiertem Fasudil}

Die Querschnittstudie dient auch zur Untersuchung der Medikamentenverträglichkeit. Es sollen mögliche Effekte durch Dosierungsunterschiede und durch eine Kombinationstherapie auf folgende Parameter beurteilt werden: die Anzahl der AlphaMotoneurone im lumbalen Rückenmark pro Vorderhorn und das Ausmaß der Muskelatrophie des Musculus gastrocnemius.

Dabei soll die Frage beantwortet werden, ob Fasudil in einer niedrigeren Dosierung (10 $\mathrm{mg} / \mathrm{kg} \mathrm{KG}$ ) vorteilhafter wirkt als in einer mittleren (30 mg/kg KG). Die Effekte von Fasudil sollen mit denen von Riluzol (30 $\mathrm{mg} / \mathrm{kg} \quad \mathrm{KG})$ sowie mit einer Kombinationstherapie beider Präparate verglichen werden. Bei der Kombination wird Fasudil konsequenterweise in der niedrigen Dosierung verabreicht.

Im Rahmen der vorliegenden Studie kann kein Präparat einen protektiven Effekt auf das Überleben der Motoneurone zeigen. Auf der Ebene des zellulären Motoneuronüberlebens zeigt zwar keine Therapie einen schädigenden Einfluss, jedoch auch keine Verbesserung des Motoneuronüberlebens.

Im Hinblick auf die Muskelatrophie kann ein signifikanter Unterschied zwischen gesunden (Wt) und kranken (Veh) Tieren festgestellt werden. Behandlungserfolge im Vergleich zur unbehandelten transgenen Kontrollgruppe erzielen die Präparate Fasudil in beiden Dosierungen und die Riluzol-Monotherapie. Die Tiere der Fas10-Gruppe und die der Ril30-Gruppe weisen einen genauso hohen Muskelanteil wie die gesunde WtKontrollgruppe auf. Im Vergleich zur gesunden Wt-Kontrollgruppe ist der Muskelanteil am Körpergewicht bei den Tieren, die mit Fasudil (30 mg/kg KG), und denen, die mit der Kombinationsbehandlung behandelt werden, signifikant gemindert, jedoch im Vergleich zur unbehandelten signifikant erhöht. Keinen Effekt zeigt die Kombinationstherapie aus Fasudil (10 mg/kg KG) und Riluzol. Es ergibt sich zudem ein signifikant niedrigerer Muskelanteil bei der Fas10Ril30-Gruppe im Vergleich zur Ril30-Gruppe $(p=0,034)$ und im Vergleich zu Fas10-Gruppe ( $\mathrm{p}=0,021)$.

Der mangelnde therapeutische Effekt der Kombinationsbehandlung deutet darauf hin, dass die protektiven Effekte der einzeln verabreichten Präparate in kombinierter Verabreichung wieder aufgehoben werden könnten. Diese Beobachtung widerspricht bisherigen Erkenntnissen über die molekularen Wirkmechanismen beider Präparate, die eine synergistische Wirkung einer Kombination nahelegen (siehe Kapitel 4.2.1). Deshalb ist es 
möglich, dass bisher unbekannte wechselseitige Wirkmechanismen die protektiven Effekte bei gemeinsamer Verabreichung blockieren. Als gemeinsamer Gegenspieler beider Präparate kann Caspase 3 ausgemacht werden (Boston-Howes et al. 2006, Jacobs et al. 2006, Coleman et al. 2001). Um die molekularen Mechanismen und mögliche Wechselwirkungen einer Kombiantionsbehandlung molekularbiologisch beurteilen zu können, ist der Einfluss auf die Glutamat-Clearance und die Expression von EAAT2 eine sinnvolle Fragestellung für nachfolgende Studien (siehe Kapitel 4.2.1).

Ein anderer entscheidender Faktor könnte die Dosierung von Fasudil sein. Es ist denkbar, dass Fasudil in einer höheren Dosierung kombiniert mit Riluzol in der Lage ist, die bekannten molekularen Synergiemechanismen besser zu nutzen. Eine ausbalancierte Gabe beider Präparate sollte das Ziel in der Zukunft nachfolgender Untersuchungen sein.

\subsection{Fazit zur Therapie mit niedrig dosiertem Fasudil und in Kombination mit Riluzol}

Ein wesentliches Ziel der vorliegenden Arbeit ist es, die Effekte einer niedrig dosierten Fasudil-Therapie und in Kombination mit Riluzol zu untersuchen. Im Rahmen der Verlaufsstudie zeigt Fasudil in der Dosierung $10 \mathrm{mg} / \mathrm{kg}$ KG keinen Einfluss auf das Überleben, die klinische Entwicklung (Körpergewicht und klinisch-neurologischer Score) und die motorkoordinativen Fähigkeiten (Drehwalzenversuch). Die niedrige Dosierung ist der höheren (30 mg/kg KG) dabei insofern unterlegen, als diese milde den Verfall motorkoordinativer Fähigkeiten aufhält (siehe Kapitel 3.1.2). Hierfür sprechen auch die Ergebnisse der videobasierten Ganganalyse: Fasudil (10 $\mathrm{mg} / \mathrm{kg} \mathrm{KG}$ ) verbessert im Gegensatz zu Fasudil (30 mg/kg KG) nicht die allgemeine motorische Leistungsfähigkeit, nicht die Lähmung und nicht den funtionellen Gebrauch der Hinterläufe in der letzten Krankheitsphase. Jedoch kann Fasudil (10 mg/kg KG) und auch in der Kombination mit Riluzol die Kraft und die Beweglichkeit aller Extremitäten über den gesamten Krankheitsverlauf verbessern.

Im Rahmen der Querschnittstudie kann Fasudil (10 mg/kg KG) einen protektiven Effekt auf die Muskeltrophik zeigen. Jedoch verliert sich dieser in der Kombinationbehandlung mit Riluzol. Fasudil in der höheren Dosierung und auch Riluzol einzeln haben günstige Effekte auf die Muskeltrophik.

Bisherige Untersuchungen zu den Wirkmechanismen von Fasudil und Riluzol lassen eine synergistische Wirkung auf der Ebene von gemeinsamen Effektor-Molekülen vermuten. Durch eine Stimulierung von EAAT2 wirken beide der Exzitotoxizität entgegen. Dass 
dieser Effekt bei einer gemeinsamen Verabreichung fehlt, ist bemerkenswert. Diese Beobachtung bedarf weiterer Untersuchungen in vitro und in vivo:

In vitro könnte die Untersuchung der Kombinationstherapie auf extrazelluläre GlutamatSpiegel und auf die Expression von EAAT2 zu weiteren Erkenntnissen auf molekularer Ebene führen. Als gemeinsamer Modulator der Wirkung beider Präparate sollte der Einfluss von Caspase 3 auf diese Prozesse beobachetet werden. Eine nähere Beleuchtung dieser Mechanismen könnte Einfluss auf das derzeitige Krankheitsverständnis haben und auf mögliche neue therapeutische Angriffspunkte hinweisen.

Die bessere Wirksamkeit von Fasudil in der Dosierung $30 \mathrm{mg} / \mathrm{kg}$ KG legt nahe, dass diese Dosierung auch in der Kombination mit Riluzol überlegen sein könnte. Dies sollte in vivo geprüft werden. Die gemeinsame Verabreichung erscheint vor dem Hintergrund der bisher bekannten molekularbiologischen Wirkmechanismen vielversprechend. Möglicherweise ist eine ausbalancierte gemeinsame Verabreichung wirksamer als eine jeweilige Mono-Therapie (siehe Kapitel 4.2.1). Dies gilt insbesondere vor dem Hintergrund der günstigen Effekte, die Fasudil in der Dosierung $30 \mathrm{mg} / \mathrm{kg}$ KG auf die motorischen Parameter der vorliegenden Studie hat. So kann als ein wesentliches Fazit dieser Studie geschlossen werden, dass Fasudil in der Dosierung von $30 \mathrm{mg} / \mathrm{kg}$ KG der niedrigen Dosierung 10 $\mathrm{mg} / \mathrm{kg}$ KG in seinem protektiven Effekt auf die motorische Performance überlegen ist. 


\section{$5 \quad$ Zusammenfassung}

Die ALS ist eine schwere, progressiv verlaufende neurodegenerative Erkrankung mit infauster Prognose. In den letzten Jahren wurden zwar Fortschritte im Verständnis der Ätiopathogenese erzielt, allerdings liegt trotz jahrelanger Forschungsbemühungen mit dem Präparat Riluzol bisher nur ein beim Menschen etabliertes therapeutisches Pharmakon vor. Riluzol ist jedoch lediglich in der Lage, das Überleben um wenige Monate zu verlängern. Gegenstand aktueller Forschung zur Therapie der ALS ist daher die Suche nach effektiveren Neuroprotektiva. In verschiedenen Modellen neurodegenerativer Erkrankungen wurde ein neuroprotektiver Effekt der Inhibition der Rho Kinase (ROCK) beobachtet. Der ROCK-Inhibitor Fasudil ist aufgrund der bereits am Menschen gezeigten Verträglichkeit dabei in den Fokus gerückt. Im SOD1G93A-Mausmodell der ALS konnte in vorherigen Studien durch eine Fasudil-Behandlung ein Gesamtüberlebensvorteil, ein verbessertes Überleben der Alpha-Motoneurone im lumbalen Rückenmark und auch eine verbesserte motorische Funktion im motorkoordinativen Drehwalzenversuch nachgewiesen werden. Dabei konnte insgesamt eine bessere Wirksamkeit einer Dosierung von 30 mg/kg KG im Vergleich zu 100 mg/kg KG beobachtet werden. In der aktuellen Arbeit sollten daher Therapieeffekte einer niedrig dosierten Therapie mit Fasudil Dosierung von $10 \mathrm{mg} / \mathrm{kg} \mathrm{KG}$ sowie die Kombination mit Riluzol untersucht werden.

Die Untersuchungen wurden methodisch am SOD1G93A-Mausmodell in Form einer klinischen Verlaufsstudie und einer histologischen Querschnittstudie durchgeführt. Neben einer gesunden Wildtyp-Gruppe und einer unbehandelten, aber erkrankten Kontrollgruppe wurden vier therapeutisch behandelte SOD1G93A-Kohorten untersucht. Geprüft und verglichen wurde die Wirkung von niedrig dosiertem Fasudil (10 mg/kg KG), Fasudil (30 mg/kg KG), Riluzol (30 mg/kg KG) und die Kombination Fasudil (10 mg/kg KG) und Riluzol (30 mg/kg KG). In der klinischen Verlaufsstudie wurde das Gesamtüberleben und die klinische Entwicklung anhand von Körpergewicht und klinisch-neurologischem Score evaluiert. Zudem wurde die motorische Performance mittels Drehwalzenversuch (Rotarod) und einer videobasierten Ganganalyse (CatWalk XT) untersucht. Die kognitive Entwicklung wurde mithilfe des novel object recognition Tests bewertet.

Resultierend zeigte sich, dass Fasudil $30 \mathrm{mg} / \mathrm{kg}$ KG die motorische Performance im motorkoordinativen Drehwalzenversuch (Rotarod) verbessert. In der videobasierten Ganganalyse (CatWalk XT) verbessert sich zudem die allgemeine motorische Leistungsfähigkeit und die Lähmung, der funktionelle Gebrauch, die Beweglichkeit und 
Kraft der Hinterläufe in der letzten Krankheitsphase. Die niedrige Dosierung mit Fasudil $10 \mathrm{mg} / \mathrm{kg}$ ermöglichte, die Kraft und Beweglichkeit der Vorder- und Hinterläufe der ALSMäuse über den gesamten Untersuchungszeitraum auf dem Niveau der gesunden Tiere zu halten. In der Kombinationsbehandlung von Fasudil mit Riluzol stellten sich ähnliche Effekte dar, die aber in der Wirksamkeit nicht überlegen waren. Keine der Behandlungen wies einen signifikanten Einfluss auf das Überleben auf. In der histologischen Querschnittstudie am Lebenstag 120 stellte sich in den Therapiegruppen kein verbessertes Überleben der Alpha-Motoneurone dar. Eine Atrophie des Musculus gastrocnemius wurde durch Fasudil in der niedrigen Dosierung und ebenso durch Riluzol vollständig verhindert. Zusammenfassend kann festgehalten werden, dass sich in dieser Studie auch für die niedrige Dosierung von Fasudil $10 \mathrm{mg} / \mathrm{kg}$ eine vorteilhafte Veränderung motorischer Parameter im SOD1G93A-Modell darstellt und somit das Potential der ROCKInhibitionstherapien bei ALS bestätigt. Eine Kombinationstherapie von Fasudil mit Riluzol weist keine additiven Effekte auf, so dass die durch Fasudil und Riluzol gemeinsam beeinflußten molekularen Mechanismen detailliert untersucht werden müssen, um verbesserte Therapieansätze zu erarbeiten. 


\section{$6 \quad$ Literaturverzeichnis}

Achterkamp D, de Keijzer R, Zimmermann P: Reference Manual Catwalk XT 10.0. Noldus, Wageningen 2012

Aguila MA del, Longstreth WT Jr, McGuire V, Koepsell TD, van Belle G (2003): Prognosis in amyotrophic lateral sclerosis: a population- based study. Neurology $\underline{60}$, 813819

Al-Chalabi A, Hardiman O (2013): The epidemiology of ALS: a conspiracy of genes, environment and time. Nat Rev Neurol $\underline{9}, 617-28$

Andersen PM (2006): Amyotrophic lateral sclerosis associated with mutations in the CuZn superoxide dismutase gene. Curr Neurol Neurosci Rep $\underline{6}$, 37-46

Andersen PM, Borasio GD, Dengler R, Hardiman O, Kollewe K, Leigh PN, Pradat PF, Silani V, Tomik B (2005): EFNS task force on management of amyotrophic lateral sclerosis: guidelines for diagnosing and clinical care of patients and relatives. Eur J Neurol $\underline{12}, 921-938$

Antunes M, Biala G (2012): The novel object recognition memory: neurobiology, test procedure, and its modifications. Cogn Process $\underline{13}$, 93-110

Aran FA (1850): Recherches sur une maladie non encore décrite du système musculaire (atrophie musculaire progressive). Arch Gen Med 14, 5-35, 172- 214

Armon C (2009): Smoking may be considered an established risk factor for sporadic ALS. Neurology $\underline{73}, 1693-1698$

Asano T, Suzuki T, Tsuchiya M, Satoh S, Ikegaki I, Shibuya M, Suzuki Y, Hidaka H (1989): Vasodilator actions of HA1077 in vitro and in vivo putatively mediated by the inhibition of proteinkinase. Br J Pharmacol 모, 1091-1100

Avraham KB, Schickler M, Sapoznikov D, Yarom R, Groner Y (1988): Down's syndrome: Abnormal neuromuscular junction in tongue of transgenic mice with elevated levels of human $\mathrm{Cu} / \mathrm{Zn}$-superoxide dismutase. Cell $\underline{54}, 823-829$ 
Avraham KB, Sugarman H, Rotshenker S, Groner Y (1991): Down's syndrome: morphological remodelling and increased complexity in the neuromuscular junction of transgenic CuZn-superoxide dismutase mice. J Neurocytol 20, 208-215

Bauer, PO, Wong HK, Oyama F, Goswami A, Okuno M, Kino Y, Miyazaki H, Nukina N (2009): Inhibition of Rho kinases enhances the degradation of mutant huntingtin. J Biol Chem $\underline{284}, 13153-13164$

Bensimon G, Lacomblez L, Meininger V and the ALS/Riluzole Study Group (1994): A controlled trial of Riluzole in amyothrophic lateral sclerosis. N Engl J Med $\underline{330}$, 585-591

Blumenstein L: Rho-Effektor-Interaktion: Struktur-Funktionsbeziehungen. Chem. Diss. Bochum 2004

Borchelt DR, Lee MK, Slunt HS, Guarnieri M, Xu ZS, Wong PC, Brown Jr. RH, Price DL, Sisodia SS, Cleveland DW (1994): Superoxide dismutase 1 with mutations linked to familial amyotrophic lateral sclerosis possesses significant activity. Proc Natl Acad Sci USA 91, 8292-8296

Borchelt DR, Guarnierie M, Wong PC, Lee MK, Slunt HS, Xu ZS, Sisodia SS, Price DL, Cleveland DW (1995): Superoxide dismutase 1 subunits with mutations linked to famlial amyotrophic lateral sclerosis do not affect wild-type subunit function. J Biol Chem 270 , 3234-3238

Boston-Howes W, Gibb SL, Williams EO, Pasinelli P, Brown RH Jr, Trotti D (2006): Caspase- 3 cleaves and inactivates the glutamate transporter EAAT2. J Biol Chem 281, 14076-84

Bowerman M, Shafey D, Kothary R (2007): Smn depletion alters profilin II expression and leads to upregulation of the RhoA/ROCK pathway and defects in neuronal integrity. J Mol Neurosci $\underline{32}, 120-131$

Bowerman M, Beauvais A, Anderson CL, Kothary R (2010): Rho-kinase inactivation prolongs survival of an intermediate SMA mouse model. Hum Mol Genet $\underline{19}, 1468-1478$

Bowerman M, Murray LM, Boyer JG, Anderson CL, Kothary R (2012): Fasudil improves survival and promotes skeletal muscle development in a mouse model of spinal muscular atrophy. BMC Med $\underline{10}, 24$ 
Braak H, Brettschneider J, Ludolph AC, Lee VM, Trojanowski JQ, Del Tredici K (2013) Amyotrophic lateral sclerosis--a model of corticofugal axonal spread. Nat Rev Neurol $\underline{9}$, $708-14$

Bradley WG, Mash DC (2009): Beyond Guam: the cyanobacteria/BMAA hypothesis of the cause of ALS and other neurodegenerative diseases. Amyotroph Lateral Scler $\underline{2}, 7-20$

Branchu J, Biondi O, Chali F, Collin T, Leroy F, Mamchaoui K, Makoukji J, Pariset C, Lopes P, Massaad C (2013): Shift from extracellular signal-regulated kinase to AKT/cAMP response element-binding protein pathway increases survival-motor-neuron expression in spinal-muscular-atrophy-like mice and patient cells. J Neurosci $\underline{33}, 4280-4294$

Brettschneider J, Del Tredici K, Toledo JB, Robinson JL, Irwin DJ, Grossman M, Suh E, Van Deerlin VM, Wood EM, Baek Y (2013): Stages of pTDP-43 pathology in amyotrophic lateral sclerosis. Ann Neurol 74, $20-38$.

Brooks BR (1994): El Escorial World Federation of Neurology criteria for the diagnosis of amyotrophic lateral sclerosis. Subcommittee on Motor Neuron Diseases/Amyotrophic Lateral Sclerosis of the World Federation of Neurology Research Group on Neuromuscular Diseases and the El Escorial "Clinical limits of amyotrophic lateral sclerosis" workshop contributors. J Neurol Sci 124, 96-107

Brooks BR, Miller RG, Swash M, Munsat TL (2000): El Escorial revisited: revised criteria for the diagnosis of amyotrophic lateral sclerosis. Amyotroph Lateral Scler Other Motor Neuron Disord 1, 293-299

Bruijn LI, Becher MW, Lee MK, Anderson KL, Jenkins NA, Copeland NG, Sisodia SS, Rothstein JD, Borchelt DR, Price DL, Cleveland DW (1997): ALS-linked SOD1 mutant G85R mediates damage to astrocytes and promotes rapidly progressive disease with SOD1containing inclusions. Neuron $\underline{18}, 327-38$

Cedarbaum JM, Stambler N (1997): Performance of the Amyotrophic Lateral Sclerosis Functional Rating Scale (ALSFRS) in multicenter clinical trials. J Neurol Sci 152, 1-9

Cedarbaum JM, Stambler N, Malta E, Fuller C, Hilt D, Thurmond B, Nakanishi A (1999): The ALSFRS-R: a revised ALS functional rating scale that incorporates assessments of respiratory function. J Neurol Sci $\underline{169}, 13-21$ 
Chancellor AM, Warlow CP (1992) Adult onset motor neuron disease: worldwide mortality, incidence and distribution since 1950. Journal of Neurology, Neurosurgery, and Psychiatry $\underline{55}, 1106-1115$

Charcot JM (1874): De la sclérose latérale amyotrophique. Prog Med 2, 325-327, 341-342, $453-455$

Charcot JM, Joffory A (1869): Deux cas d'atrophie musculaire progressive avec lésions de la substance grise et des faisceaux antéro-lateraux de la moelle épinière. Arch Physiol Neurol Pathol 2, 744-754

Chen M, Liu A, Ouyang Y, Huang Y, Chao X, Pi R (2013): Fasudil and its analogs: a new powerful weapon in the long war against central nervous system disorders? Expert Opin Investig Drugs $\underline{22}, 537-550$

Chiò A, Logroscino G, Hardiman O, Swingler R, Mitchell D, Beghi E, Traynor BG (2009): Prognostic factors in ALS: A critical review. Amyotroph Lateral Scler 10, 310-323

Chiò A, Logroscino G, Traynor BJ, Collins J, Simeone JC, Goldstein LA, White LA (2013): Global Epidemiology of Amyotrophic Lateral Sclerosis: a Systematic Review of the Published Literature. Neuroepidemiology $\underline{41}, 118-130$

Chiu AY, Zhai P, Canto MC Dal, Peters TM, Kwon YW, Prattis SM, Gurney ME (1995): Age- dependent penetrance of disease in a transgenic mouse model of familial amyotrophic lateral sclerosis. Mol Cell Neurosci $\underline{6}$, 349-362

Coleman ML, Sahai EA, Yeo M, Bosch M, Dewar A, Olson MF (2001): Membrane blebbing during apoptosis results from caspasemediated activation of ROCK I. Nat Cell Biol $\underline{3}, 339-45$

Costa J, Swash M, Carvalho M de (2012): Awaji Criteria for the Diagnosis of Amyotrophic Lateral Sclerosis: A Systematic Review. Arch Neurol 69, 1-7

Cruveilhier J (1853): Sur le paralysie musculaire progressive atrophique. Bull Acad Med $\underline{18}$, 490-501, 546-583

Cudkowicz ME, van den Berg LH, Shefner JM, Mitsumoto H, Mora JS, Ludolph A, Hardiman O, Bozik ME, Ingersoll EW, Archibald D (2013): Dexpramipexole versus placebo for patients with amyotrophic lateral sclerosis (EMPOWER): a randomised, double-blind, phase 3 trial. Lancet Neurol 12, 1059-1067 
Dal Canto MC, Gurney ME (1995): Neuropathological changes in two lines of mice carrying a transgene for mutant human $\mathrm{Cu}, \mathrm{Zn} \mathrm{SOD}$, and in mice overexpressing wild type human SOD: a model of familial amyotrophic lateral sclerosis (FALS). Brain Res $\underline{676}$, 2540

Davies SP, Reddy H, Caivano M, Cohen P (2000): Specificity and mechanism of action of some commonly used protein kinase inhibitors. Biochem J $\underline{351}$, 95-105

de Carvalho M, Dengler R, Eisen A, England JD, Kaji R, Kimura J, Mills K, Mitsumoto H, Nodera H, Shefner J, Swash M (2008): Electrodiagnostic criteria for diagnosis of ALS. Clin Neurophysiol 119, 497-503

Dellu F, Contarino A, Simon H, Koob GF, Gold LH (2000): Genetic differences in response to novelty and spatial memory using a two-trial recognition task in mice. Neurobiol Learn Mem 73(1), 31-48

Deyts C, Galan-Rodriguez B, Martin E, Bouveyron N, Roze E, Charvin D, Caboche J, Bétuing S (2009): Dopamine D2 Receptor Stimulation Potentiates PolyQ-HuntingtinInduced Mouse Striatal Neuron Dysfunctions via Rho/ROCK-II Activation. PLoS ONE 4(12): e8287

DGN-Leitlinie 2012 Amyotrophe Lateralsklerose (Motoneuronerkrankungen). In: Leitlinien für Diagnostik und Therapie in der Neurologie: Herausgegeben von der Kommission "Leitlinien" der Deutschen Gesellschaft für Neurologie. 5. Auflage; hrsg. v. Diener HC, Weimar C unter Mitarbeit namhafter Autoren; Georg Thieme Verlag KG, Stuttgart 2012, vollständig überarbeitet: 1. Juni 2014, online auf www.dgn.org seit: 13. Februar 2015, gültig bis: 31. Mai 2019

Doble A, Hubert JP, Blanchard JC (1992): Pertussis toxin pretreatment abolishes the inhibitory effect of riluzole and carbachol on D-[\%] aspartate release from cultured cerebellar granule cells. Neurosci Lett $\underline{140}$, 251-254

Duchenne de Boulogne GB (1851): Recherches électrophysiologiques et thérapeutiques. Comp Rend Seances Acad Sci $\underline{32}$, 506-510

Eisen A, Schulzer M, MacNeil M, Pant B, Mak E (1993): Duration of amyotrophic lateral sclerosis is age dependent. Muscle Nerve $\underline{16}, 27-32$ 
Epstein C J, Avraham KB, Lovett M, Smith S, Elroy-Stein O, Rotman G, Bry C, Groner Y (1987): Transgenic mice with increased $\mathrm{Cu} / \mathrm{Zn}$-superoxide dismutase activity: animal model of dosage effects in Down syndrome. Proc Natl Acad Sci U S A $\underline{84}, 8044-8048$

Farah S, Agazie Y, Ohan N, Ngsee JK, Liu XJ (1998): A rho-associated protein kinase, ROKalpha, binds insulin receptor substrate-1 and modulates insulin signaling. J Biol Chem $\underline{273}, 4740-6$

Fischer LR, Culver DG, Tennant P, Davis AA, Wang M, Castellano-Sanchez A, Khan J, Polak MA, Glass JD (2004): Amyotrophic lateral sclerosis is a distal axonopathy: evidence in mice and man. Exp Neurol $\underline{185}, 232-240$

Foran E, Trotti D (2009): Glutamate Transporters and the Excitotoxic Path to Motor Neuron Degeneration in Amyotrophic Lateral Sclerosis. Antioxid Redox Signal 11, $1587-$ 1602

Fumagalli E, Funicello M, Rauen T, Gobbi M, Mennini T (2008): Riluzole enhances the activity of glutamate transporters GLAST, GLT1 and EAAC1. Eur J Pharmacol $\underline{578}, 171$ 176

Gerber YN, Sabourin JC, Rabano M, Vivanco Md, Perrin FE (2012): Early functional deficit and microglial disturbances in a mouse model of amyotrophic lateral sclerosis. PLoS One $\underline{I}$ (4): e 36000

Gould TW, Buss RR, Vinsant S, Prevette D, Sun W, Knudson CM, Milligan CE, Oppenheim RW (2006): Complete Dissociation of Motor Neuron Death from Motor Dysfunction by Bax Deletion in a Mouse Model of ALS. The Journal of Neuroscience 26, 8774

Grad LI, Guest WC, Yanai A, Pokrishevsky E, O'Neill MA, Gibbs E, Semenchenko V, Yousefi M, Wishart DS, Plotkin SS, Cashman NR (2011): Intermolecular transmission of superoxide dismutase 1 misfolding in living cells. Proc Natl Acad Sci U S A $\underline{108}$, 16398-403

Guillot TS, Asress SA, Richardson JR, Glass JD, Miller GW (2008): Treadmill gait analysis does not detect motor deficits in animal models of Parkinson's disease or amyotrophic lateral sclerosis. J Mot Behav $\underline{40}, 568-77$

Günther R: Pharmakologische Inhibition von Rho-Kinase im Mausmodell der Amyotrophen Lateralsklerose. Med. Diss. Göttingen 2015 
Günther R, Suhr M, Koch JC, Bähr M, Lingor P, Tönges L (2012): Clinical Testing and Spinal Cord Removal in a Mouse Model for Amyotrophic Lateral Sclerosis (ALS). J Vis $\operatorname{Exp} \underline{61}, 3936$

Günther R, Saal KA, Suhr M, Scheer D, Koch JC, Bähr M, Lingor P, Tönges L (2014): The rho kinase inhibitor Y-27632 improves motor performance in male SOD1(G93A) mice. Front Neurosci $\underline{8}, 304$

Günther R, Balck A, Koch JC, Nientiedt T, Sereda M, Bähr M, Lingor P, Tönges L (2017): Rho Kinase Inhibition with Fasudil in the SOD1G93A Mouse Model of Amyotrophic Lateral Sclerosis-Symptomatic Treatment Potential after Disease Onset. Front Pharmacol $\underline{8}, 17$

Gurney ME, Pu H, Chiu AY, Canto MC Dal, Polchow CY, Alexander DD, Caliendo J, Hentati A, Kwon YW, Deng HX (1994): Motor neuron degeneration in mice that express a human $\mathrm{Cu}, \mathrm{Zn}$ superoxide dismutase mutation. Science 264, 1772-1775

Hall ED, Oostveen JA, Gurney ME (1998): Relationship of microglial and astrocytic activation to disease onset and progression in a transgenic model of familial ALS. Glia $\underline{23}$, 249-256

Hardiman O, van den Berg LH, Kiernan MC (2011): Clinical diagnosis and management of amyotrophic lateral sclerosis. Nat Rev Neurol I, 639-649.

Hashimoto R, Nakamura Y, Kosako H, Amano M, Kaibuchi K, Inagaki M (1999): Distribution of Rho-kinase in the bovine brain. Biochem Biophys Res Commun 263, 5759

Hayashi H, Kato S (1989): Total manifestations of amyotrophic lateral sclerosis: ALS in the totally locked-in state. J Neurol Sci $\underline{93}, 19-35$

Heiman-Patterson TD, Deitch JS, Blankenhorn EP, Erwin KL, Perreault MJ, Alexander BK, Byers N, Toman I, Alexander GM (2005): Background and gender effects on survival in the $\operatorname{TgN}(S O D 1-G 93 A) 1 G u r$ mouse model of ALS. J Neurol Sci 236, 1-7

Henkel JS, Beers DR, Zhao W, Appel SH (2009): Microglia in ALS: the good, the bad, and the resting. J Neuroimmune Pharmacol $\underline{4}, 389-98$ 
Hensel N, Stockbrugger I, Rademacher S, Broughton N, Brinkmann H, Grothe C, Claus P (2014): Bilateral crosstalk of rho- and extracellular- signal-regulated-kinase (ERK) pathways is confined to an unidirectional mode in spinal muscular atrophy (SMA). Cell Signal $\underline{26}$, $540-548$

Hensel N, Rademacher S, Claus P (2015): Chatting with the neighbors: crosstalk between Rho-kinase (ROCK) and other signaling pathways for treatment of neurological disorders. Front Neurosci $\underline{9}, 198$

Herbert T, Drapeau P, Pradier L, Dunn FLJ (1994): Block of the rat brain IIA sodium channel a subunit by the neuroprotective drug riluzole. Mol Pharmacol 45, 1055-1060

Herskowitz JH, Feng Y, Mattheyses AL, Hales CM, Higginbotham LA, Duong DM, Montine TJ, Troncoso JC, Thambisetty M, Seyfried NT (2013): Pharmacologic Inhibition of ROCK2 Suppresses Amyloid- $\beta$ Production in an Alzheimer's Disease Mouse Model. J Neurosci $\underline{33}, 19086-19098$

Hubert JP, Delumeau JC, Glowinski J, Promont J, Doble A (1994): Antagonism by riluzole of entry of calcium evoked by NMDA and veratridine in rat cultured granule cells: Evidence for a dual mechanism of action. Br J Pharmacol $\underline{113}$, 261-267

Ingre C, Landers JE, Rizik N, Volk AE, Akimoto C, Birve A, Hübers A, Keagle PJ, Piotrowska K, Press R (2013): A novel phosphorylation site mutation in profilin 1 revealed in a large screen of US, Nordic, and German amyotrophic lateral sclerosis/frontotemporal dementia cohorts. Neurobiol Aging $\underline{34}, 1708$ e1-6

Jacobs M, Hayakawa K, Swenson L, Bellon S, Fleming M, Taslimi P, Doran J (2006): The structure of dimeric ROCK I reveals the mechanism for ligand selectivity. J Biol Chem $\underline{281}$, $260-8$

Jaffe AB, Hall A (2005): Rho GTPases: biochemistry and biology. Annu Rev Cell Dev Biol $\underline{21}, 247-69$

Johnston JA, Dalton MJ, Gurney ME, Kopito RR (2000): Formation of high molecular weight complexes of mutant $\mathrm{Cu}, \mathrm{Zn}$-superoxide dismutase in a mouse model for familial amyotrophic lateral sclerosis. Proc Natl Acad Sci U S A 7, 12571-12576

Kaminski HJ, Richmonds CR, Kusner LL, Mitsumoto H (2002): Differential susceptibility of the ocular motor system to disease. Ann N Y Acad Sci 256, 42-54 
Kawano Y, Fukata Y, Oshiro N, Amano M, Nakamura T, Ito M. (1999): Phosphorylation of myosin-binding subunit (MBS) of myosin phosphatase by Rho-kinase in vivo. J Cell Biol $\underline{147}, 1023-38$

Kiernan MC, Vucic S, Cheah BC, Turner MR, Eisen A, Hardiman O, Burrell JR, Zoing MC (2011): Amyotrophic lateral sclerosis. Lancet $\underline{377}$, 942-955

Kimura K, Ito M, Amano M, Chihara K, Fukata Y, Nakafuku M (1996): Regulation of myosin phosphatase by Rho and Rho-associated kinase (Rho-kinase). Science $\underline{273}$, 245-8

Kimura K, Fukata Y, Matsuoka Y, Bennett V, Matsuura Y, Okawa K (1998): Regulation of the association of adducin with actin filaments by Rho-associated kinase (Rho-kinase) and myosin phosphatase. J Biol Chem $\underline{273}, 5542-8$

Knippenberg S, Thau N, Dengler R, Petri S (2010): Significance of behavioural tests in a transgenic mouse model of amyotrophic lateral sclerosis (ALS). Behav Brain Res $\underline{213}$, 8287

Körner S, Petri S, Dengler R, Kollewe K (2011): Amyotrophe Lateralsklerose. Nervenheilkunde $\underline{30}, 755-763$

Lacomblez L, Bensimon G, Leigh PN, Guillet P, Powe L, Durrleman S, Delumeau JC, Meininger V and the ALS/Riluzole Study Group II (1996): A confirmatory dose- ranging study of riluzole in ALS. Neurology $\underline{47}, 242-250$

Lacorte E, Ferrigno L, Leoncini E, Corbo M, Boccia S, Vanacore N (2016): Physical activity, and physical activity related to sports, leisure and occupational activity as risk factors for ALS: A systematic review. Neurosci Biobehav Rev $\underline{66}, 61-79$

Lau CL, O'Shea RD, Broberg BV, Bischof L, Beart PM (2011): The Rho kinase inhibitor Fasudil up-regulates astrocytic glutamate transport subsequent to actin remodelling in murine cultured astrocytes. Br J Pharmacol $\underline{163}, 533-45$

Lee SE (2011): Guam dementia syndrome revisited in 2011. Curr Opin Neurol 24, 517-524 Leitlinie ALS: s. DGN-Leitlinie 2012

Li M, Huang Y, Ma AA, Lin E, Diamond MI (2009): Y-27632 improves rotarod performance and reduces huntingtin levels in R6/2 mice. Neurobiol Dis $\underline{36}$, 413-420 
Li YH, Yu JZ, Liu CY, Zhang H, Zhang HF, Yang WF, Li JL, Feng QJ, Feng L, Zhang GX (2014): Intranasal delivery of FSD-C10, a novel Rho kinase inhibitor, exhibits therapeutic potential in experimental autoimmune encephalomyelitis. Immunology $\underline{143}$, 219-229

Lingor P, Teusch N, Schwarz K, Mueller R, Mack H, Bähr M, Mueller BK (2007): Inhibition of Rho kinase (ROCK) increases neurite outgrowth on chondroitin sulphate proteoglycan in vitro and axonal regeneration in the adult optic nerve in vivo. J Neurochem $\underline{103}, 181-189$

Lingor P, Tönges L, Pieper N, Bermel C, Barski E, Planchamp V, Bähr M (2008): ROCK inhibition and CNTF interact on intrinsic signalling pathways and differentially regulate survival and regeneration in retinal ganglion cells. Brain 131, 250-263

Logroscino G, Traynor BJ, Hardiman O, Chiò A, Couratier P, Mitchell JD, Swingler RJ, Beghi E (2008): Descriptive epidemiology of amyotrophic lateral sclerosis: new evidence and unsolved issues. J Neurol Neurosurg Psychiatr $\underline{79}, 6-11$

Logroscino G, Traynor BJ, Hardiman O, Chiò A, Mitchell D, Swingler RJ, Millul A, Benn E, Beghi E (2010): Incidence of amyotrophic lateral sclerosis in Europe. J Neurol

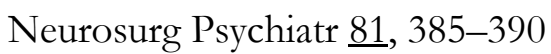

Ludolph AC: Amyotrophe Lateralsklerose (ALS). In: Berlit P (Hrsg.): Klinische Neurologie, 2. Auflage; Springer-Verlag, Heidelberg 2006, 541-550

Ludolph AC, Bendotti C, Blaugrund E, Chiò A, Greensmith L, Loeffler JP, Mead R, Niessen HG, Petri S, Pradat PF (2010): Guidelines for preclinical animal research in ALS/MND: A consensus meeting. Amyotroph Lateral Scler 11, 38-45

Ludolph AC, Drory V, Hardiman O, Nakano I, Ravits J, Robberecht W, Shefner J (2015): A revision of the El Escorial criteria - 2015. Amyotroph Lateral Scler Frontotemporal Degener 16, 291-292

Maekawa M, Ishizaki T, Boku S, Watanabe N, Fujita A, Iwamatsu A (1999): Signaling from Rho to the actin cytoskeleton through protein kinases ROCK and LIM-kinase. Science $\underline{285}$, 895-8

Mancuso R, Oliván S, Osta R, Navarro X (2011): Evolution of gait abnormalities in SOD1(G93A) transgenic mice. Brain Res 1406, 65-73 
Mannen T (2000): Neuropathological findings of Onuf's nucleus and its significance. Neuropathology 20, $30-33$

Matsui T, Maeda M, Doi Y, Yonemura S, Amano M, Kaibuchi K (1998): Rho-kinase phosphorylates $\mathrm{COOH}$ - terminal threonines of ezrin/radixin/moesin (ERM) proteins and regulates their head-to-tail association. J Cell Biol 140, 647-57

Mead RJ, Bennett EJ, Kennerley AJ, Sharp P, Sunyach C, Kasher P, Berwick J, Pettmann B, Battaglia G, Azzouz M (2011): Optimised and rapid pre-clinical screening in the SOD1(G93A) transgenic mouse model of amyotrophic lateral sclerosis (ALS). PLoS One $\underline{6}$ (8): e23244

Meyer T, Münch C, Landeghem van FKH, Borisow N, Dullinger J, Linke P (2007): Progressive muscle atrophy. Nervenarzt $\underline{78}, 1383-1388$

Miller RG, Mitchell JD, Moore DH (2012): Riluzole for amyotrophic lateral sclerosis (ALS)/motor neuron disease (MND). Cochrane Database of Systematic Reviews Issue $\underline{3}$. Art. No.: CD001447

Minc-Golomb D, Knobler H, Groner Y (1991): Gene dosage of CuZnSOD and Down's syndrome: diminished prostaglandin synthesis in human trisomy 21, transfected cells and transgenic mice. EMBO 10, 2119-2124

Mohajeri MH, Figlewicz DA, Bohn MC (1998): Selective loss of alpha motoneurons innervating the medial gastrocnemius muscle in a mouse model of amyotrophic lateral sclerosis. Exp Neurol 150, 329-336

Mourelatos Z, Gonatas NK, Stieber A, Gurney ME, Canto MC Dal (1996): The Golgi apparatus of spinal cord motor neurons in transgenic mice expressing mutant $\mathrm{Cu}, \mathrm{Zn}$ superoxide dismutase becomes fragmented in early, preclinical stages of the disease. Proc Natl Acad Sci U S A $\underline{93}, 5472-5477$

Münch C, O'Brien J, Bertolotti A (2011): Prion-like propagation of mutant superoxide dismutase-1 misfolding in neuronal cells. Proc Natl Acad Sci U S A $\underline{108}, 3548-53$

Nagumo H, Sasaki Y, Ono Y, Okamoto H, Seto M, Takuwa Y (2000): Rho kinase inhibitor HA-1077 prevents Rho-mediated myosin phosphatase inhibition in smooth muscle cells. Am J Physiol Cell Physiol 278, C57-65 
Nakagawa O, Fujisawa K, Ishizaki T, Saito Y, Nakao K, Narumiya S (1996): ROCK-I and ROCK-II, two isoforms of Rho-associated coiled-coil forming protein serine/threonine kinase in mice. FEBS Lett $\underline{392}$, 189-93

Ng J, Luo L (2004): Rho GTPases regulate axon growth through convergent and divergent signaling pathways. Neuron $\underline{44}, 779-793$

O'Rourke JG, Bogdanik L, Yáñez A, Lall D, Wolf AJ, Muhammad AK, Ho R, Carmona S, Vit JP, Zarrow J (2016): C9orf72 is required for proper macrophage and microglial function in mice. Science $\underline{351}, 1324-1329$

Pasinelli P, Houseweart MK, Brown RH Jr, Cleveland DW (2000): Caspase-1 and -3 are sequentially activated in motor neuron death in $\mathrm{Cu}, \mathrm{Zn}$ superoxide dismutase-mediated familial amyotrophic lateral sclerosis. Proc Natl Acad Sci U S A 무, 13901-6

Perlson E, Jeong GB, Ross JL, Dixit R, Wallace KE, Kalb RG, Holzbaur EL (2009): A switch in retrograde signaling from survival to stress in rapid-onset neurodegeneration. J Neurosci 29, 9903-17

Pratt J, Rataud J, Bardot F, Roux M, Blanchard JC, Laduron PM, Stutzmann JM (1992): Neuroprotective actions of riluzole in rodent models of global and focal cerebral ischaemia. Neuroscience Letters 140, 225-230.

Proud CG (2007): A sharper instrument for dissecting signalling events: a specific AGC kinase inhibitor. Biochem J 401, 1-3.

Ravits J, Paul P, Jorg C (2007): Focality of upper and lower motor neuron degeneration at the clinical onset of ALS. Neurology $\underline{68}, 1571-1575$

Renton AE, Majounie E, Waite A, Simón-Sánchez J, Rollinson S, Gibbs JR, Schymick JC, Laaksovirta H, van Swieten JC, Myllykangas L (2011): A hexanucleotide repeat expansion in C9ORF72 is the cause of chromosome 9p21-linked ALS-FTD. Neuron $\underline{72}$, 257-68

Riento K, Ridley AJ (2003): Rocks: multifunctional kinases in cell behaviour. Nat Rev Mol Cell Biol 4, 446-56

Ringholz GM, Appel SH, Bradshaw M, Cooke NA, Mosnik DM, Schulz PE (2005): Prevalence and patterns of cognitive impairment in sporadic ALS. Neurology $\underline{65}, 586-590$ 
Riviere M, Meininger V, Zeisser P, Munsat T (1998): An Analysis of Extended Survival in Patients With Amyotrophic Lateral Sclerosis Treated With Riluzole. Arch Neurol $\underline{55}, 526$ 528.

Román GC (1996): Neuroepidemiology of amyotrophic lateral sclerosis: clues to aetiology and pathogenesis. Journal of Neurology, Neurosurgery, and Psychiatry $\underline{61}, 131-137$

Rosen DR, Siddique T, Patterson D, Figlewicz DA, Sapp P, Hentati A, Donaldson D, Goto J, O'Regan JP, Deng HX (1993): Mutations in Cu/Zn superoxide dismutase gene are associated with familial amyotrophic lateral sclerosis. Nature $\underline{362}, 59-62$

Rothstein JD (1996): Therapeutic horizons for amyotrophic lateral sclerosis. Curr Opin Neurobiol $\underline{6}, 679-687$

Rothstein, JD (2017): Edaravone: A new drug approved for ALS. Cell 171, 725

Rothstein JD, Kuncl RW (1995): Neuroprotective strategies in a model of chronic glutamate-mediated motor neuron toxicity. J Neurochem $\underline{65}, 643-51$

Rowland LP (2001): How amyotrophic lateral sclerosis got its name: The clinical-pathologic genius of jean-martin charcot. Arch Neurol $\underline{58}, 512-515$

Rowland LP, Shneider NA (2001): Amyotrophic lateral sclerosis. N Engl J Med $\underline{344}$, 16881700

Rübsamen S, Lücking CH: Degenerative Erkrankungen der Motoneurone, Amytrophe Lateralsklerose (ALS). In: Hufschmidt A, Lücking CH, Rauer S: Neurologie compact. Für Klinik und Praxis. 6. Auflage; Thieme, Stuttgart 2013, 328-332

Saal KA, Koch JC, Tatenhorst L, Szegö EM, Ribas VT, Michel U, Bähr M, Tönges L, Lingor P (2015): AAV.shRNA-mediated downregulation of ROCK2 attenuates degeneration of dopaminergic neurons in toxin-induced models of Parkinson's disease in vitro and in vivo. Neurobiol Dis $\underline{73}, 150-62$

Sabatelli M, Madia F, Conte A, Luigetti M, Zollino M, Mancuso I, Lo Monaco M, Lippi G, Tonali P (2008): Natural history of young-adult amyotrophic lateral sclerosis. Neurology 71, 876-881

Sabatelli M, Zollino M, Luigetti M, Grande AD, Lattante S, Marangi G, Monaco ML, Madia F, Meleo E, Bisogni G (2011): Uncovering amyotrophic lateral sclerosis phenotypes: 
Clinical features and long-term follow-up of upper motor neuron-dominant ALS. Amyotroph Lateral Scler 12, 278-282

Sarmiere PD, Bamburg JR (2004): Regulation of the neuronal actin cytoskeleton by ADF/cofilin. J Neurobiol 토, 103-117

Sasaki Y, Sasaki Y, Kanno K, Hidaka H (1987): Disorganization by calcium antagonists of actin micro-filament in aortic smooth muscle cells. Am J Physiol 253, C71-C78

Saxena S, Cabuy E, Caroni P (2009): A role for motoneuron subtype-selective ER stress in disease manifestations of FALS mice. Nat Neurosci 12, 627-36

Schickler M, Knobler H, Avraham KB, Elroy-Stein O, Groner Y (1989): Diminished serotonin uptake in platelets of transgenic mice with increased $\mathrm{Cu} / \mathrm{Zn}$-superoxide dismutase activity. EMBO $\underline{8}, 1385-1392$

Schiller MR (2006): Coupling receptor tyrosine kinases to Rho GTPases-GEFs what's the link. Cell Signal $\underline{18}, 1834-43$

Schmandke A, Schmandke A, Strittmatter SM (2007): ROCK and Rho: biochemistry and neuronal functions of Rho-associated protein kinases. Neuroscientist $\underline{13}$, 454-469

Schreiber H, Gaigalat T, Wiedemuth-Catrinescu U, Graf M, Uttner I, Muche R, Ludolph AC (2005): Cognitive function in bulbar- and spinal onset amyotrophic lateral sclerosis - a longitudinal study in 52 patients. J Neurol 252, 772-81.

Schroder HD, Reske-Nielsen E (1984): Preservation of the nucleus X-pelvic floor motosystem in amyotrophic lateral sclerosis. Clin Neuropathol $\underline{3}, 210-16$

Shababi M, Lorson CL, Rudnik-Schoneborn SS (2014): Spinal muscular atrophy: a motor neuron disorder or a multi-organ disease? J Anat $\underline{224}, 15-28$

Sorarù G, Ermani M, Logroscino G, Palmieri A, D' Ascenzo C, Orsetti V, Volpe M, Cima V, Zara G, Pegoraro E (2010): Natural history of upper motor neuron-dominant ALS. Amyotroph Lateral Scler 11, 424-429

Spalloni A, Geracitano R, Berretta N, Sgobio C, Bernardi G, Mercuri NB, Longone P, Ammassari-Teule M (2006): Molecular and synaptic changes in the hippocampus underlying superior spatial abilities in pre-symptomatic G93A+/+ mice overexpressing the human $\mathrm{Cu} / \mathrm{Zn}$ superoxide dismutase (Gly93 --> ALA) mutation. Exp Neurol 197, 505-14. 
Suhr M: Effekte der präsymptomatischen Applikation der Rho-Kinase-Inhibitoren Fasudil und Y-27632 im SOD1(G93A)-Mausmodell der Amyotrophen Lateralsklerose. Med. Diss. Göttingen 2017

Sun X, Minohara M, Kikuchi H, Ishizu T, Tanaka M, Piao H, Osoegawa M, Ohyaghei Y, Shimokawa H, Kira J (2006): The selective Rho-kinase inhibitor Fasudil is protective and therapeutic in experimental autoimmune encephalomyelitis. J Neuroimmunol $\underline{180}, 126-134$

Sun Y, Chakrabartty A (2017): Phase to Phase with TDP-43. Biochemistry $\underline{56}$, 809-823

Sutedja NA, Veldink JH, Fischer K, Kromhout H, Heederik D, Huisman MH, Wokke JH, van den Berg LH (2009): Exposure to chemicals and metals and risk of amyotrophic lateral sclerosis: A systematic review. Amyotroph Lateral Scler 10, 302-309

Takata M, Tanaka H, Kimura M, Nagahara Y, Tanaka K, Kawasaki K, Seto M, Tsuruma K, Shimazawa M, Hara H (2013): Fasudil, a rho kinase inhibitor, limits motor neuron loss in experimental models of amyotrophic lateral sclerosis. Br J Pharmacol $\underline{170}$, 341-351

Takayasu M, Suzuki Y, Shibuya M, Asano T, Kanamori M, Okada T, Kageyama N, Hidaka H (1986): The effects of HA compound calcium antagonists on delayed cerebral vasospasm in dogs. J Neurosurg $\underline{65}, 80-85$

Talbot K, Ansorge O (2006): Recent advances in the genetics of amyotrophic lateral sclerosis and frontotemporal dementia: common pathways in neurodegenerative disease. Hum Mol Genet $\underline{15}, 182-187$

Talbott EO (2016): The epidemiology of amyotrophic lateral sclerosis. Handb Clin Neurol $\underline{138}, 225-238$

Taylor JP, Brown RH Jr, Cleveland DW (2016): Decoding ALS: from genes to mechanism. Nature $\underline{539}, 197-206$

Tönges L, Frank T, Tatenhorst L, Saal KA, Koch JC, Szegö ÉM, Bähr M, Weishaupt JH, Lingor P (2012): Inhibition of rho kinase enhances survival of dopaminergic neurons and attenuates axonal loss in a mouse model of Parkinson's disease. Brain $\underline{135}, 3355-70$

Tönges L, Günther R, Suhr M, Jansen J, Balck A, Saal KA, Barski E, Nientied T, Götz AA, Koch JC, Mueller BK, Weishaupt JH, Sereda MW, Hanisch UK, Bähr M, Lingor P (2014): Rho kinase inhibition modulates microglia activation and improves survival in a model of amyotrophic lateral sclerosis. Glia $\underline{62}, 217-232$ 
Tu PH, Raju P, Robinson KA, Gurney ME, Trojanowski JQ, Lee VM (1996): Transgenic mice carrying a human mutant superoxide dismutase transgene develop neuronal cytoskeletal pathology resembling human amyotrophic lateral sclerosis lesions. Proc Natl Acad Sci U S A $\underline{93}, 3155-3160$

Turner BJ, Talbot K (2008): Transgenics, toxicity and therapeutics in rodent models of

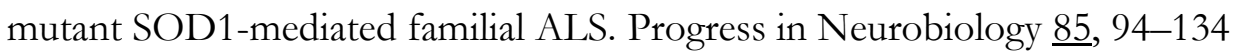

Tyler HR, Shefner J (1991): Amyotrophic lateral sclerosis. Handb Clin Neurol 15, 169-215

Vergouts M, Marinangeli C, Ingelbrecht C, Genard G, Schakman O, Sternotte A, Calas AG, Hermans E (2015): Early ALS-type gait abnormalities in AMP-dependent protein kinase-deficient mice suggest a role for this metabolic sensor in early stages of the disease. Metab Brain Dis $\underline{30}, 1369-77$

Ward Y, Yap SF, Ravichandran V, Matsumura F, Ito M, Spinelli B (2002): The GTP binding proteins Gem and Rad are negative regulators of the Rho-Rho kinase pathway. J Cell Biol 157, 291-302

Weisskopf MG, O'Reilly EJ, McCullough ML, Calle EE, Thun MJ, Cudkowicz M, Ascherio A (2005): Prospective study of military service and mortality from ALS. Neurology 64, 3237

Weydt P, Hong SY, Kliot M, Möller T (2003): Assessing disease onset and progression in the SOD1 mouse model of ALS. Neuroreport 14, 1051-1054

Wijesekera LC, Leigh PN (2009): Amyotrophic lateral sclerosis. Orphanet J Rare Dis 4, 3-3 Williamson TL, Cleveland DW (1999): Slowing of axonal transport is a very early event in the toxicity of ALS-linked SOD1 mutants to motor neurons. Nat Neurosci 2 , 50-6

Wolfrum S, Dendorfer A, Rikitake Y, Stalker TJ, Gong Y, Scalia R (2004): Inhibition of Rho-kinase leads to rapid activation of phosphatidylinositol 3-kinase/protein kinase Akt and cardiovascular protection. Arterioscler Thromb Vasc Biol 24, 1842-7

Wooley CM, Sher RB, Kale A, Frankel WN, Cox GA, Seburn KL (2005): Gait analysis detects early changes in transgenic SOD1(G93A) mice. Muscle Nerve $\underline{32}$, 43-50

Worms PM (2001): The epidemiology of motor neuron diseases: a review of recent studies. J Neurol Sci 191, 3-9 
Wu CH, Fallini C, Ticozzi N, Keagle PJ, Sapp PC, Piotrowska K, Lowe P, Koppers M, McKenna-Yasek D, Baron DM (2012): Mutations in the profilin 1 gene cause familial amyotrophic lateral sclerosis. Nature $\underline{488}, 499-503$

Zaldivar, T. Gutierrez J, Lara G, Carbonara M, Logroscino G, Hardiman O (2009): Reduced frequency of ALS in an ethnically mixed population: a population-based mortality study. Neurology $\underline{72}, 1640-1650$.

Zang DW, Yang Q, Wang HX, Egan G, Lopes EC, Cheema SS (2004): Magnetic resonance imaging reveals neuronal degeneration in the brainstem of the superoxide dismutase 1G93A G1H transgenic mouse model of amyotrophic lateral sclerosis. Eur J Neurosci 20, 1745-1751

Zhang H, Li Y, Yu J, Guo M, Meng J, Liu C, Xie Y, Feng L, Xiao B, Ma C (2013): Rho kinase inhibitor fasudil regulates microglia polarization and function. Neuroimmunomodulation 20, 313-322

Zhou Y, Su Y, Li B, Liu F, Ryder JW, Wu X, Gonzalez-DeWhitt PA, Gelfanova V, Hale JE, May PC (2003): Nonsteroidal anti-inflammatory drugs can lower amyloidogenic Abeta42 by inhibiting Rho. Science $\underline{302}, 1215-7$ 


\section{Danksagung}

Besonderen Dank möchte ich an Herrn Prof Dr. med. Lars Tönges und Herrn Prof. Dr. med. Paul Lingor für das Überlassen des Promotionsthemas richten. Ohne ihren außergewöhnlichen Einsatz und ihre professionelle Betreuung wäre meine Dissertation nicht möglich gewesen. Ich danke Herrn Prof. Dr. med. Mathias Bähr, die Infrastruktur des ihm unterstehenden Bähr-Lab genutzt haben zu können. Ohne die Einwerbung finanzieller Mittel durch den Arbeitsgruppenleiter Herrn Prof. Dr. med. Paul Lingor wäre dieses kostenintensive Projekt zudem nicht möglich gewesen. Dafür möchte ich mich sehr bedanken.

Besonderen Dank richte ich an Frau Elisabeth Barski, die mir bei der Durchführung der Genotypisierung und der immunhistochemischen Färbung stets zur Seite gestanden hat. Ebenso Frau Vivian Dambeck und Herrn Dr. rer. nat. Lars Tatenhorst, die mir beim Erlernen und Durchführen der verhaltensbiologischen Versuche und der Gewebepräparation immer zur Seite standen. Ich bedanke mich dabei auch bei den Mitarbeitern der ZTE für ihre unschätzbare Arbeit, besonders zu nennen ist hier Herr Michael Blessmann.

Ich bedanke mich bei Herrn Dr. med. Rene Günther und Herrn Dr. med. Martin Suhr, die die Arbeit am Mausmodell der ALS zusammen mit Herrn Prof. Dr. med. Lars Tönges in unserer Arbeitsgruppe etabliert haben und ohne deren Erkenntnisse meine Arbeit nicht vorstellbar gewesen wäre. Ebenso gilt mein Dank Herrn Alexander Balck für die Beratung bei der Durchführung des kognitiven Tests und der Gewebepräparation.

Ich danke zudem allen Mitgliedern der Arbeitsgruppe von Prof. Dr. med. Paul Lingor, die mir stets in unzähligen Labormeetings Rat geben konnten und mir die Zeit im Labor auch neben dem wissenschaftlichen Arbeiten sehr angenehm gemacht haben. Besonders zu nennen sind hier Frau Dr. rer. nat. Kim-Ann Saal und Frau Dr. rer. nat. Elisa Roser. 


\section{Lebenslauf}

Ich wurde am 13.01.1990 in Göttingen geboren. Die allgemeine Hochschulreife erlangte ich durch das Abitur im Jahr 2009 am Felix-Klein-Gymnasium Göttingen. Weil ich den Dienst an der Waffe verweigerte, leistete ich darauf meinen neunmonatigen Wehrersatzdienst auf der unfallchirurgischen Normalstation 5013 an der Universitätsmedizin Göttingen. Im Sommersemester 2010 nahm ich an der Georg-AugustUniversität Göttingen das Studium der Humanmedizin auf. Den Ersten Abschnitt der Ärztlichen Prüfung bestand ich im April 2012, den Zweiten im Oktober 2015, den Dritten am 9. November 2016 und schloss das Studium mit der Ärztlichen Prüfung ab, Gesamtnote: gut. Famulaturen leistete ich in der Kinderheilkunde, speziell Kinderkardiologie, in der unfallchirurgischen Notfallaufnahme und in der Neurologie an der Universitätsmedizin Göttingen sowie in der Anästhesiologie des Krankenhauses NeuBethlehem in Göttingen. Während des Studiums sammelte ich darüber hinaus praktische Erfahrungen als studentische Hilfskraft im Pflegepool der Universitätsmedizin Göttingen, von Mai 2010 bis August 2012, im Zentrum Anatomie im Präparierkurs der makroskopischen Anatomie im Wintersemester 2011/12, in der anästhesiologischen Pflege im Aufwachbereich des Krankenhauses Neu-Bethlehem in Göttingen, von August 2012 bis Dezember 2015, sowie im Rufdienst der Klinik für Unfallchirurgie, Orthopädie und Plastische Chirurgie an der Universitätsmedizin Göttingen, von Februar 2014 bis Dezember 2015. Mein Praktisches Jahr leistete ich am Klinikum Oldenburg, Klinik für Innere Medizin, am Universitätsklinikum Köln, Klinik für Chirurgie und am Universitätsklinikum Münster, Klinik für Neurologie.

Daneben arbeitete ich seit Februar 2013 in der Arbeitsgruppe von Prof. Dr. med. Lingor im Bähr-Lab in der Klinik für Neurologie an der Universitätmedizin Göttingen an meiner Promotion, der vorliegenden Arbeit. In diesem Rahmen erwarb ich ein den Federation of European Laboratory Animal Science Associations (FELASA)-B-Richtlinien entsprechendes Zertifikat, Tierversuche durchführen zu dürfen.

Seit August 2017 befinde ich mich an der Universitätsklinik für Innere Medizin Onkologie und Hämatologie am Klinikum Oldenburg in der Weiterbildung zum Facharzt für Innere Medizin und Hämatologie und Onkologie. 